\title{
USING AMBIENT SEISMIC NOISE TO STUDY TEMPORAL AND SPATIAL SURFACE WAVE VELOCITY STRUCTURES AND AMBIENT NOISE FIELD CHARACTERISTICS OF CENTRAL SOUTH ISLAND, NEW ZEALAND
}

\author{
by \\ RACHEL ELIZABETH GREENE HECKELS \\ A thesis \\ submitted to the Victoria University of Wellington \\ in fulfilment of the requirements for the degree of \\ Doctor of Philosophy \\ in Geophysics.
}





\section{Abstract}

Ambient seismic noise is used to examine the spatial and temporal surface wave velocity structures and ambient seismic noise fields in the vicinity of different fault zone environments. This study focuses on two distinct regions of central South Island, New Zealand. The Canterbury Plains is a sedimentary basin with many minor faults, which was considered to have low seismic hazard prior to the $2010-$ 2011 Canterbury earthquake sequence. We focus on the time period immediately following the 2010 Darfield earthquake, which ruptured the previously unmapped Greendale Fault. The second region of interest is the central Southern Alps. The locked portion of the Alpine Fault currently poses one of the largest seismic hazards for New Zealand. The wealth of data from both permanent and temporary seismic deployments in these regions make them ideal areas in which to assess the effectiveness of ambient noise for velocity modelling in regions surrounding faults at different stages of their seismic cycles.

Temporal velocity changes are measured following the $\mathrm{M}_{w} 7.1$ Darfield earthquake of 4 September 2010 in the Canterbury Plains. Nine-component cross-correlations are computed from temporary and permanent seismic stations lying on and surrounding the Greendale Fault. Using the Moving-Window Cross-Spectral method, surface wave velocity changes are calculated for the four months immediately following the earthquake until 10 January 2011, for 0.1 - $1.0 \mathrm{~Hz}$. An average increase in seismic velocity of $0.14 \pm 0.04 \%$ is determined throughout the region, providing the first such estimate of postseismic relaxation rates in Canterbury. Depth analyses further showed that velocity changes are confined to the uppermost $5 \mathrm{~km}$ of the subsurface and we attribute this to postseismic relaxation via crack-healing of the Greendale Fault and throughout the surrounding region.

Rayleigh and Love wave dispersion is examined throughout the Canterbury region. Multi-component cross-correlation functions are analysed for group and phase dispersion curves. These are inverted using frequency-time analysis for 2-D phase and group velocity maps of Rayleigh and Love waves. A high-velocity zone to the southeast of the region coincides with volcanic rocks of Banks Peninsula. Dispersion curves generated from the surface wave tomography are further inverted for one- 
dimensional shear velocity profiles. These models show a thin, low-velocity near surface layer consistent with the basin sediments, which thins towards the foothills of the Southern Alps. A near-surface damage zone is identified along the length of the Greendale Fault, with consistent reduced $V_{S}$ velocities to depth of up to 5 $\mathrm{km}$.

Surface and shear wave velocity maps are computed for the central Southern Alps to image the seismic structure of the region. Tomographic surface maps at periods of $5-12 \mathrm{~s}$ are produced from dispersion measurements of three-component cross-correlation functions. At periods of $5-8 \mathrm{~s}$ a strong NE-SW trending velocity contrast highlights the Alpine Fault. One-dimensional shear velocity models, computed from the surface wave maps, are in agreement with previous models produced by other conventional methods. An analysis of surface wave amplitudes through signal-to-noise ratios of cross-correlations reveals strong directional effects. Calculated signal-to-noise ratios are up to eight times higher for surface waves travelling north-west than for waves travelling to the south or east. We attribute this to a combination of more energetic ocean wave signals from the Southern Ocean compared to the Tasman Sea. 


\section{Acknowledgements}

There are many people to thank for helping me create this piece of work; be it through professional, scientific and academic support, or emotional and physical support and encouragement.

I would like to thank both my supervisors Martha and John for their guidance and support over the last four years. Thank you for the late night proof-reads, keeping me calm when things going wrong and getting me through my $\mathrm{PhD}$. A huge thank you to Aleks and Adrian for their technical help and for putting up with my constant stream of silly computing questions. Heartfelt thanks goes to Patricia Stein, Kate King and Miranda Voke for always being there to help with the admin side of things.

Thank you to all the funding bodies who provided me with financial support throughout my PhD; Victoria University for the Doctoral Scholarship, The Royal Society of New Zealand Marsden Fund, The Geoscience Society of New Zealand Jim Ansell Scholarship. Thank you to the organisations that have helped me attend many conferences and workshops; the Geoscience Society of New Zealand, the New Zealand Earthquake Commission and the American Geophysical Union.

A huge thank you to my fellow Geophysics students at Victoria, past and present, in particular Laura-May, Dom, Konrad, Jesse, Katrina, Adrian and Holly for keeping things fun at uni and eating cake and drinking tea with me. To Francesco and Andy, our ambient noise discussions and your help with computing has been invaluable.

To all my wonderful friends, near and far, thanks for keeping me down here on Earth. Especially Marilou and Athene, Erin and Leonie. To Gina, Briony, Ruth and the rest of my Sutton Girls and Sarah, Heather and Kat of the Yorkshire Tea Crew thank you for being the best friends anyone could ask for.

A short but well-deserved shout-out to my Wadestown GirlGuides. You're always there on a Thursday evening with bags of enthusiasm and energy, and bluntly honest wisdom only 10 year old girls can provide. No matter how disheartened my work 
might make me, I always left Guides with a huge grin on my face and warm fuzzies inside!

Last, but most definitely not least, thank you Mom, Dad and Alex. Your unwavering, unconditional love and support has got me through these last four years. Despite the miles and time zones you've always been there whenever I need a laugh, cry or a moan. You put up with/laugh at my craziness, my clumsiness, my complaining and my grumpiness (especially in the last few weeks). Thank you to my Grandad Billie for keeping me up to date with the gossip at home and hiding the chocolate and biscuits just for me. I would like to include my cat Fitz here, for making me get up and out of bed on time every morning, even if he did only want his salmon for breakfast, but I've been warned that stating this on paper would only serve to cement my 'crazy cat lady' status!

I would like to dedicate this thesis to my Grandmas Pat and Ida. You were two of the strongest women I know and you constantly inspired and encouraged me to follow my wildest dreams and grab all opportunities that came my way through life. Everything you did and worked for was to give us grandchildren the best lives possible. I hope I've made you both proud. 


\section{Contents}

Abstract $\quad$ i

Acknowledgements

List of Figures ix

List of Tables $\quad$ xiii

Abbreviations and Nomenclature $\quad$ Xv

1 Introduction $\quad 1$

1.1 Motivations ........................ 1

1.2 Scope of the research . . . . . . . . . . . . . . 2

1.3 Thesis overview . . . . . . . . . . . . . . . . . . 3

1.4 Tectonics and basement geology of South Island, New Zealand . . . . 5

1.5 Previous ambient seismic noise studies in New Zealand . . . . . . . . 8

2 Theoretical background and methodology 11

2.1 Ambient noise theory . . . . . . . . . . . . . . . . . . . . . . 11

2.1.1 Surface waves and ambient seismic noise . . . . . . . . . . . 11

2.1.2 Cross-correlation technique and seismic velocity retrieval . . . 13

2.1.3 Noise directionality across central South Island . . . . . . . . 16

2.1.4 Temporal velocity variations . . . . . . . . . . . . . 16

2.2 Pre-processing . . . . . . . . . . . . . . . . . . . . . . . . . 21

2.2.1 Instrument response removal and polarities . . . . . . . . . . 22

2.2.2 Spectral whitening . . . . . . . . . . . . . . 24

2.2.3 Time-domain normalisation _. . . . . . . . . . . . . 24

2.3 Cross-correlation computation . . . . . . . . . . . . . . 26

2.4 Velocity modelling . . . . . . . . . . . . . . . . . . 27

2.4.1 Surface wave dispersion . . . . . . . . . . . . . . . . 27

2.4 .2 Surface wave tomography . . . . . . . . . . . . . . 28

2.4.3 Shear wave inversion . . . . . . . . . . . . . . . 30 
2.5 Velocity variations: Moving-window cross-spectral analysis . . . . . . 31

2.6 Quality control and error analysis . . . . . . . . . . . . . . 32

3 Velocity models of Canterbury 35

3.1 Introduction . . . . . . . . . . . . . . . . 35

3.2 Data . . . . . . . . . . . . . . . . . 36

3.2.1 Data preparation . . . . . . . . . . . . 37

3.3 Methods . . . . . . . . . . . . . . . . . 39

3.3.1 Cross-correlation computation . . . . . . . . . . . . . . 39

3.3.2 Synthetic cross-correlation functions and higher mode surface waves .......................... 43

3.3.3 Canterbury dispersion . . . . . . . . . . . . . 52

3.3.4 Surface wave tomography . . . . . . . . . . . . . 58

3.3 .5 Shear wave inversion . . . . . . . . . . . . . . . 62

3.4 Results . . . . . . . . . . . . . . . . . . . . . 64

3.4.1 Rayleigh group and phase velocity maps . . . . . . . . . . . 64

3.4.2 Love group and phase velocity maps . . . . . . . . . . . . 69

3.4.3 Surface wave sensitivity kernels . . . . . . . . . . . . . 70

3.4.4 1-D shear velocity models . . . . . . . . . . . . . . 71

3.5 Discussion . . . . . . . . . . . . . . . . . . 72

4 Time-Varying Properties of Surface Waves $\quad 79$

4.1 Abstract . . . . . . . . . . . . . . . . . . . . . . 79

4.2 Introduction . . . . . . . . . . . . . . . . . . 79

4.3 Data and Methods . . . . . . . . . . . . . . . . . . 82

4.4 Results . . . . . . . . . . . . . . . . . . 86

4.5 Discussion ........................... 87

4.6 Acknowledgements . . . . . . . . . . . . . . . . 96

5 Velocity Models of the central Southern Alps $\quad 97$

5.1 Introduction . . . . . . . . . . . . . . . . . . . . . . 97

5.2 Data . . . . . . . . . . . . . . . . . 99

5.2.1 Data Preparation . . . . . . . . . . . . . 100

5.2.2 Rotation of Raw Horizontal Components . . . . . . . . . . . . 100

5.3 Methods . . . . . . . . . . . . . . . . . . . . . . . 102

5.3.1 Cross-correlation Computation . . . . . . . . . . . 103

5.3.2 Alpine Region Dispersion . . . . . . . . . . . . . . . . . 106

5.3 .3 Surface wave tomography . . . . . . . . . . . . . . . 108

5.3 .4 Shear wave inversion . . . . . . . . . . . . . . . . 110

5.4 Results . . . . . . . . . . . . . . . . . . . . . 110 
5.4.1 Rayleigh and Love Group Velocity Maps . . . . . . . . . . . . 111

5.4.2 Rayleigh and Love Phase Velocity Maps . . . . . . . . . . . . 112

5.4.3 Sensitivity Kernels . . . . . . . . . . . . . . . . . . . . . 112

5.4.4 Shear velocity Models . . . . . . . . . . . . . . . . . 113

5.5 Discussion . . . . . . . . . . . . . . . . . . . 114

6 Discussion and Conclusions 131

6.1 Cross-correlation quality: comparisons between study regions . . . . . 131

6.2 Summary of key findings . . . . . . . . . . . . . . . . . . 132

6.3 Recommendations for future study . . . . . . . . . . . . . . . . 134

A Temporary Network Metadata $\quad 135$

B Parameter and Starting Model Tables 139

$\begin{array}{lr}\text { Bibliography } & 139\end{array}$ 


\section{List of Figures}

1.1 Map of New Zealand's major tectonic features . . . . . . . . . . . . 6

1.2 New Zealand Basement terrances (Mortimer, 2004) . . . . . . . . . . 7

1.3 New Zealand wide surface wave tomography of Lin et al. (2007) . . . 9

2.1 Example of surface wave dispersion . . . . . . . . . . . . . . . . . 12

2.2 Primary and secondary microseisms in continuous seismic recordings . 14

2.3 Noise directionality in the Canterbury Plains . . . . . . . . . . . . . . 17

2.4 Noise directionality for the central Southern Alps . . . . . . . . . . . 18

2.5 Noise directionality of New Zealand, after Lin et al. (2007) . . . . . . 19

2.6 Flowchart of processing steps . . . . . . . . . . . . . . 23

2.7 Example amplitude and phase response curves for different instrument types . . . . . . . . . . . . . . . . . . . . . . 24

2.8 Effect of time and frequency domain normalisation on raw data . . . 26

2.9 Schematic of Signal-to-noise ratio calculation . . . . . . . . . . . . . 33

3.1 Map of Canterbury region with seismic networks . . . . . . . . . . 37

3.2 Data availability for networks in the Canterbury region . . . . . . . . 38

3.3 Effect of stack length on the SNR . . . . . . . . . . . . . . . . . . . 40

3.4 Daily RR correlation functions of station pair MQZ-DAR06 . . . . . 41

3.5 Daily RR correlation functions of station pair DAR02-DAR04 . . . . 42

3.6 Record section of stacked cross-correlation functions . . . . . . . . . . 44

3.7 Record sections of Canterbury from Savage et al. (2013) . . . . . . . . 45

3.8 High frequency cross-correlations of Canterbury . . . . . . . . . . . . 46

3.9 Low frequency cross-correlations of Canterbury . . . . . . . . . . . . 48

3.10 Synthetic Green's functions for vertical and radial components . . . . 50

3.11 Synthetic Green's functions for vertical and radial components . . . . 51

3.12 SNR's of Canterbury cross-correlations . . . . . . . . . . . . . . . . 52

3.13 Signal-to-noise ratios of Canterbury DAR stations, as a function of period ........................... 53

3.14 Comparison of SNR as a function of period, for the three crosscorrelation components . . . . . . . . . . . . . . . . 53 
3.15 Canterbury dispersion curves computed from FTAN . . . . . . . . . 55

3.16 Canterbury dispersion curves used in tomographic inversions . . . . . 56

3.17 Uncertainties in dispersion curves from monthly cross-correlations . . 57

3.18 Vertical component group velocity measurements for several station pair subsets . . . . . . . . . . . . . . . . . 5 59

3.19 Interstation path coverage for RR group tomography . . . . . . . . . 60

3.20 Spatial resolution analysis of Canterbury tomography maps . . . . . . 61

3.21 Azimuthal path coverage of Canterbury tomography maps . . . . . . 62

3.22 Canterbury Rayleigh wave group velocity maps . . . . . . . . . . . . 65

3.23 Canterbury Rayleigh wave group velocity maps from RR correlations 66

3.24 Canterbury Rayleigh wave phase velocity maps . . . . . . . . . . . 67

3.25 Canterbury Rayleigh wave phase velocity maps from RR correlations 68

3.26 Canterbury Love wave group velocity maps . . . . . . . . . . . . . . . 69

3.27 Canterbury Love wave phase velocity maps . . . . . . . . . . . . . . . 70

3.28 Sensitivity of fundamental mode surface waves . . . . . . . . . . . . . 75

3.29 1-D $\mathrm{V}_{S}$ models for $-43.6,172.3 \ldots \ldots \ldots$

3.30 1-D V $\mathrm{V}_{S}$ models for Canterbury from Rayleigh phase velocity measurements . . . . . . . . . . . . . . . . 77

4.1 Map of Canterbury region with seismic networks and an example of a stacked cross-correlation function . . . . . . . . . . . . . . 83

4.2 Vertical component cross-correlations as a function of inter-station distance. . . . . . . . . . . . . . . . . . . . 84

4.3 Example of MWCS computation . . . . . . . . . . . . . . . 88

4.4 Seismic velocity variations for the extended study period . . . . . . . 89

4.5 Examples of relative velocity variation curves for individual station

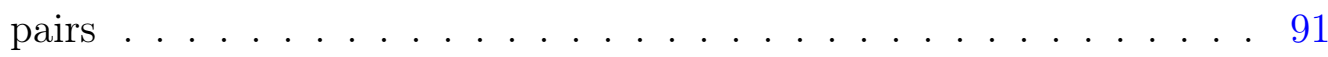

4.6 Seismic velocity variations by cross-correlation component . . . . . . 92

4.7 Modelled best fit to the velocity increase results . . . . . . . . . . . . 94

4.8 Vertical-component seismic velocity variations by frequency . . . . . . 95

5.1 Historic Alpine Fault ruptures, after Sutherland et al. (2007) . . . . . 98

5.2 Map of central Southern Alps region with seismic networks . . . . . . 99

5.3 Seismic data availability of the central Southern Alps for 2013 . . . . 101

5.4 Amplitude and phase response curves for sensors used in the Southern

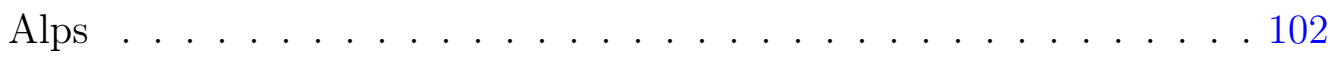

5.5 Example cross-correlation functions for station pair LBZ-WZ18 . . . 104

5.6 Nine-component positive-lag cross-correlation functions for station pair LBZ-WZ18 . . . . . . . . . . . . . . . . . . . 105 
5.7 Stability of cross-correlations through time, for example station pair LBZ-WZ18 . . . . . . . . . . . . . . . . . . 106

5.8 Histogram of SNR for all station pairs in the central Southern Alps . 107

5.9 Uncertainties in dispersion curves from monthly cross-correlations . . 108

5.10 Comparison of dispersion curves for RR, ZZ and TT components . . . 109

5.11 Interstation path coverage for RR group tomography . . . . . . . . . 117

5.12 Azimuthal path coverage of the central Southern Alps tomography maps . . . . . . . . . . . . . . . . . . . . 118

5.13 Spatial resolution analysis of central Alps tomography maps . . . . . 119

5.14 Alps Rayleigh wave Group Velocity maps . . . . . . . . . . . . . . . . 120

5.15 Alps Rayleigh wave Group Velocity maps from RR correlations . . . . 121

5.16 Alps Love wave Group Velocity maps . . . . . . . . . . . . . . . . . . 122

5.17 Alps Rayleigh wave phase velocity maps from ZZ correlations . . . . . 123

5.18 Alps Rayleigh wave phase velocity maps from RR correlations . . . . 124

5.19 Alps Love wave phase velocity maps from TT correlations . . . . . . 125

5.20 Sensitivity of fundamental mode surface waves . . . . . . . . . . . . 126

5.21 Locations of Southern Alps presented 1-D models . . . . . . . . . . . 127

5.22 1-D V $\mathrm{V}_{S}$ models for the central Southern Alps . . . . . . . . . . . . . . 128

5.23 1-D $\mathrm{V}_{S}$ models for locations $-43.6,170.1$ and $-44.1,170.2 \ldots 129$ 


\section{List of Tables}

3.1 Cross-correlation processing parameters used for the Canterbury study. 39

3.2 Multi-layer velocity model for synthetic Green's functions . . . . . . . 47

3.3 Simple 2 layer velocity model for synthetic Green's functions . . . . . 49

3.4 Canterbury $\mathrm{V}_{S}$ inversion initial model parameterisation . . . . . . . . 63

4.1 Relative velocity increases by cross-correlation component . . . . . . 90

5.1 Cross-correlation processing parameters used for the Alpine Fault study 103

5.2 Southern Alps $\mathrm{V}_{S}$ inversion initial model parameterisation . . . . . . 110

A.1 Instruments of the VUW-UWM-UA temporary network . . . . . . . . 136

A.2 Instruments of the GeoNet temporary Canterbury network . . . . . . 137

A.3 Instruments of the SAMBA network . . . . . . . . . . . . . . . . 138

B.1 Average regional velocity model of Eberhart-Phillips et al. (2010) for the Canterbury Plains region . . . . . . . . . . . . . . . . . . 140

B.2 Two-layer velocity model of (Savage et al., 2013) . . . . . . . . . . . . 140

B.3 Average regional velocity model of Eberhart-Phillips et al. (2010) for the central Southern Alps region . . . . . . . . . . . . . . . . 140

B.4 Canterbury inversion starting model . . . . . . . . . . . . . . . . . . . 141

B.5 Alps inversion starting model . . . . . . . . . . . . . . . . . . 141 


\section{Abbreviations and Nomenclature}

c Phase velocity

FTAN Frequency-Time ANalysis

H/V Horizontal-over-vertical amplitude ratio

$\mathbf{M}_{w} \quad$ Moment magnitude

MWCS Moving-window cross-spectra

NA Neighbourhood Algorithm

RMS Root-mean-squared

RR Radial-radial component

RT Radial-transverse component

RZ Radial-vertical component

SAC Seismic Analysis Code (Goldstein and Snoke, 2005)

SNR Signal-to-noise ratio

TR Transverse-radial component

TT Transverse-transverse component

TZ Transverse-vertical component

U Group velocity

$\mathrm{V}_{P} \quad$ Primary/compressional wave velocity

$\mathbf{V}_{S} \quad$ Shear-wave velocity

ZR Vertical-radial component

ZT Vertical-transverse component

ZZ Vertical-vertical component 


\section{Introduction}

\subsection{Motivations}

When considering hazards in earthquake-prone regions, knowledge of the nearsurface crustal structure is important. Accurate models of the shallow subsurface seismic velocity structure can assist in computations of the shaking to be expected during large events (e.g. Magistrale et al., 2000; Olsen et al., 2006) and are required to locate and produce moment tensor solutions of earthquakes. Many methods, both active and passive, are employed by the global seismic community to create models of the uppermost crust, such as the relatively recent development of using surface waves from ambient seismic noise analyses to retrieve shear-wave velocity, $\mathrm{V}_{S}$, structure (e.g. Behr et al., 2010; Brenguier et al., 2007; Mordret et al., 2013).

Traditional structural studies that rely on earthquakes are limited by the spatial and temporal distribution of events, which are largely constrained to plate boundaries. These studies are furthermore limited by the spatial distribution of seismic stations, which are almost always confined to land or near-shore locations. Active methods, although often costly, somewhat overcome this as they can be used on land or sea, but typically cannot produce energy magnitudes as large as earthquakes, thus can only sample small areas and typically image the uppermost crust. Since the mid2000s, ambient seismic noise has increasingly been used to image seismic velocity structures as it is not limited by the temporal or spatial distribution of earthquakes, but by the seismic networks used in studies and to a lesser degree the azimuthal distribution of ambient noise sources (Snieder, 2004). The distances between sources and receivers are commonly shorter for ambient noise studies than for teleseismic earthquakes, resulting in recorded waves experiencing less attenuation. This allows for the reliable extraction of seismic waves over a wider range of frequencies, especially short periods (Louie, 2001). The maximum usable period in ambient noise studies is dictated by the maximum interstation distance of the recording array (e.g. Bensen et al., 2007). 


\subsection{Scope of the research}

The overarching aim of this research project is to examine surface wave velocity structures and ambient seismic noise fields in different fault zone environments. The work presented in this thesis is focussed on two regions of South Island, New Zealand. The Canterbury Plains, in particular the area surrounding the fault zone of the relatively recent 2010-2011 Darfield and Christchurch earthquake sequences (Bannister and Gledhill, 2012), is examined. The second area of focus is the west coast of South Island, in the region surrounding the central locked portion of the mature Alpine Fault.

In particular, the project will seek to address the following objectives and research questions:

- Following the Darfield 2010 earthquake are there temporal changes in the seismic velocity structure or seismic noise field? If increases in seismic velocities are measured, can we attribute this to post-seismic relaxation? The movingwindow cross-spectral (MWCS) technique will be used to process data in the four months immediately following the 4th September event.

- Can high resolution surface wave and shear velocity models of Canterbury and the Central Southern Alps be obtained? Using the ambient noise crosscorrelation technique, long-duration noise records of seismic data for both study regions will be processed to extract cross-correlation functions. Group and phase velocity dispersion curves will be produced using the frequency-time analysis (FTAN) technique. The results will be presented as 2-D pseudo-depth slices at various periods. The results of the surface wave models will be inverted to create $1-\mathrm{D}$ shear wave models.

- What additional information can Rayleigh wave higher modes provide? Higher modes have been shown to be present in the Canterbury noise field (Savage et al., 2013) at high frequencies and so may be present in the lower frequencies considered in this work.

- Can known geophysical and geological features in both study regions be observed in the surface wave models? A discussion of the suitability of the ambient noise technique with respect to the regions examined will be given. 


\subsection{Thesis overview}

\section{Chapter 2: Theoretical background and methodology}

Chapter 2 presents the theory behind surface waves and how they are approximated in ambient noise cross-correlations. A literature review of previous studies and their applications is given, including surface and shear wave velocity modelling and monitoring of temporal seismic velocity changes. Descriptions of the techniques and methods implemented in this study are given, including obtaining cross-correlations, FTAN dispersion analysis, surface wave tomography and the MWCS technique for temporal velocity changes. The procedure for selecting suitable processing parameters is discussed, in addition to quality control measures used throughout this work.

\section{Chapter 3: Seismic velocity models of the Canterbury re- gion}

In this chapter we compute cross-correlation functions for a four month time period immediately following the Darfield 2010 earthquake. Continuous waveform data from two temporary rapid-response networks and several permanent GeoNet seismic stations were used. We process for surface wave dispersion curves of both Love and Rayleigh wave phases using the FTAN method. Following a tomographic inversion of surface waves, representative dispersion curves are inverted for shear-velocity models throughout the Canterbury region.

A shallow, surface layer with slow velocities exists throughout the Canterbury region, with a thickness of approximately $1-2 \mathrm{~km}$ and increasing in thickness towards Banks Peninsula. For depths to $4-8 \mathrm{~km}$, slower seismic velocities are observed in the immediate vicinity of the Greendale Fault, which is suggestive of a fault damage zone.

\section{Chapter 4: Temporal velocity changes following the 2010 Darfield Earthquake}

Chapter 4 is based on a journal article that has been formatted to be consistent with this thesis. The paper (Heckels et al., 2017), entitled Postseismic velocity variation following the $2010 M_{w} 7.1$ Darfield earthquake, New Zealand, revealed by ambient seismic noise analyses has been submitted to Geophysical Journal International and 
is currently in review. Using the moving-window cross-spectral analysis method, an increase in seismic velocities of $0.15 \pm 0.04 \%$ was observed across the Canterbury Region in the four months immediately following the Darfield event. A psuedo-depth analysis was performed, showing that temporal changes in the surface wave velocity are limited to below $5 \mathrm{~s}$ period, which equates approximately to the uppermost 5 $\mathrm{km}$.

\section{Chapter 5: Seismic velocity models of the Central Alpine Fault}

Chapter 5 determines velocity models of the central Alpine Fault region. Using several seismic networks, shear and surface wave velocity models are produced from ambient seismic noise records. The northeast-southwest trending Alpine Fault is delineated by a discrete change in surface wave speeds in both Love and Rayleigh models, with higher velocities through the Southern Alps mountain range. Onedimensional shear-wave velocity models throughout the region show little-to-no sedimentary cover at the Southern Alps, which thickens towards Canterbury. The Rayleigh and Love phase -derived models interpreted conjointly. They are critically compared to models obtained through other methods, and found to obtain similar features.

\section{Chapter 6: Discussion and Conclusions}

The final chapter of this work presents a summary of the major findings of the study. A short discussion compares the two study regions, with reference to the seismic networks and the robustness of the resulting cross-correlations and velocity models. Recommendations for future ambient seismic noise studies are suggested to conclude the thesis. 


\subsection{Tectonics and basement geology of South Is- land, New Zealand}

The continent of Zealandia (Mortimer and Campbell, 2014) lies on the boundary between the Pacific and Australian tectonic plates (Figure 1.1). The relative plate motion vector of Australia to the Pacific is currently $37 \pm 2 \mathrm{~mm}_{\mathrm{yr}} \mathrm{y}^{-1}$ at $071 \pm$ $2^{\circ}$ (DeMets et al., 1994). The physical manifestation of this motion between the plates changes along the length of New Zealand. Off the east coast of North Island, the Hikurangi Margin experiences subduction of the Pacific Plate and continues north at the Kermadec Trench. From Fiordland in the southwest of South Island and offshore, the Puysegur Trench is undergoing subduction of the Australian Plate under the Pacific. The transition between these two subduction-dominant regimes is characterised by the Alpine Fault and Marlborough Fault Zone (Walcott, 1998). The Alpine Fault accommodates oblique-convergent and strike-slip motion, where the Chatham Rise and Campbell Plateau of the Pacific Plate are converging with the Australian Challenger Plateau (Figure 1.1, Cox and Sutherland, 2007). Further north, the Marlborough Fault Zone is a system of strike-slip faults allowing for the transition to subduction in the North Island (Eberhart-Phillips et al., 2010). 


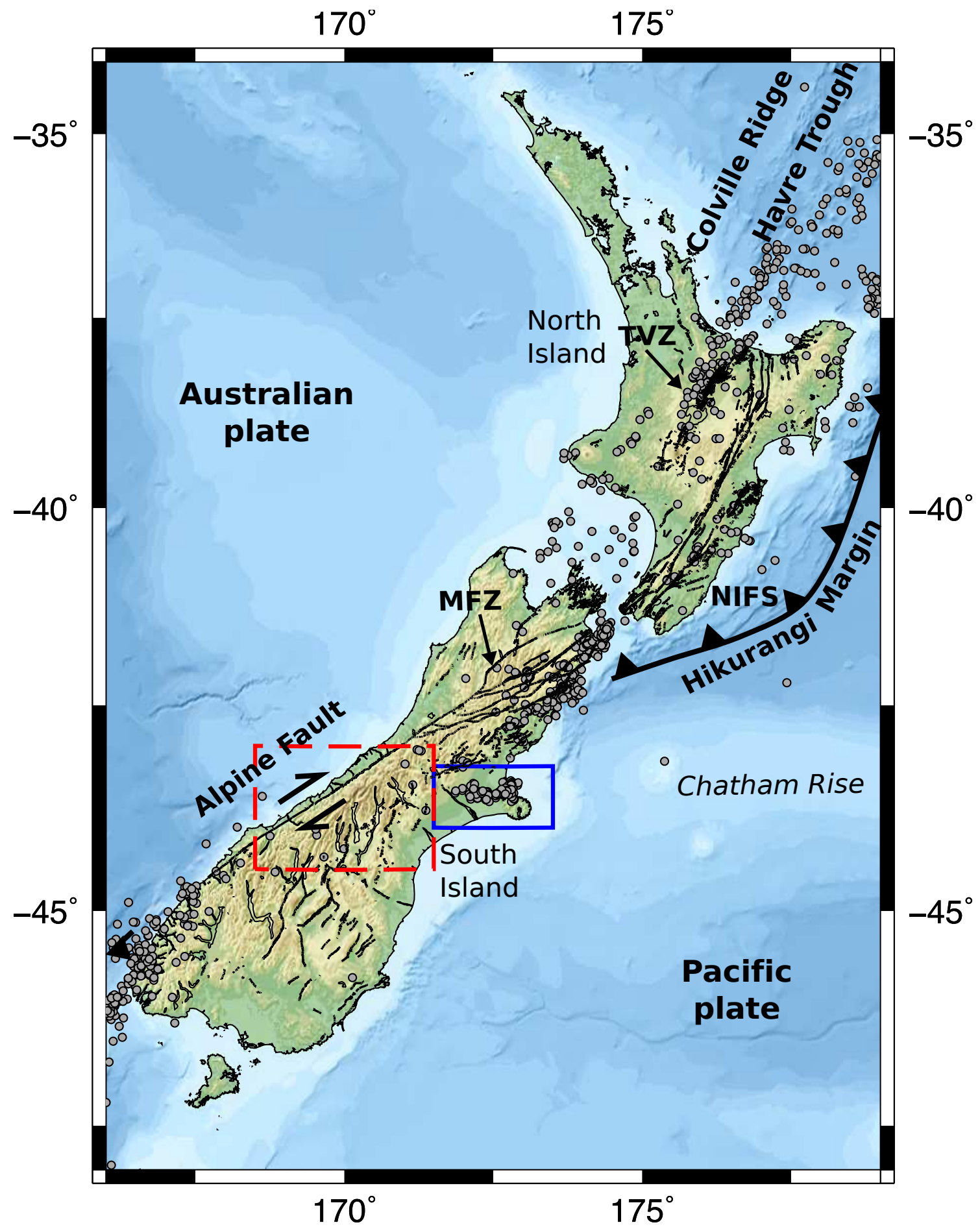

Figure 1.1: Major tectonic features of New Zealand. Thin black lines highlight the major mapped faults of New Zealand. The nature of the plate boundaries within New Zealand, with subduction of the Pacific Plate along the Hikurangi Margin, and predominantly dextral motion along the Alpine Fault. MFZ and NIFS indicate the Marlborough and North Island fault zones, and the broad southwest trending zone through central North Island is the Taupo Volcanic Zone (TVZ). Grey dots show locations of all $\mathrm{M}_{w}>4.5$ recorded in New Zealand for the 10 years from 2007-2017. 


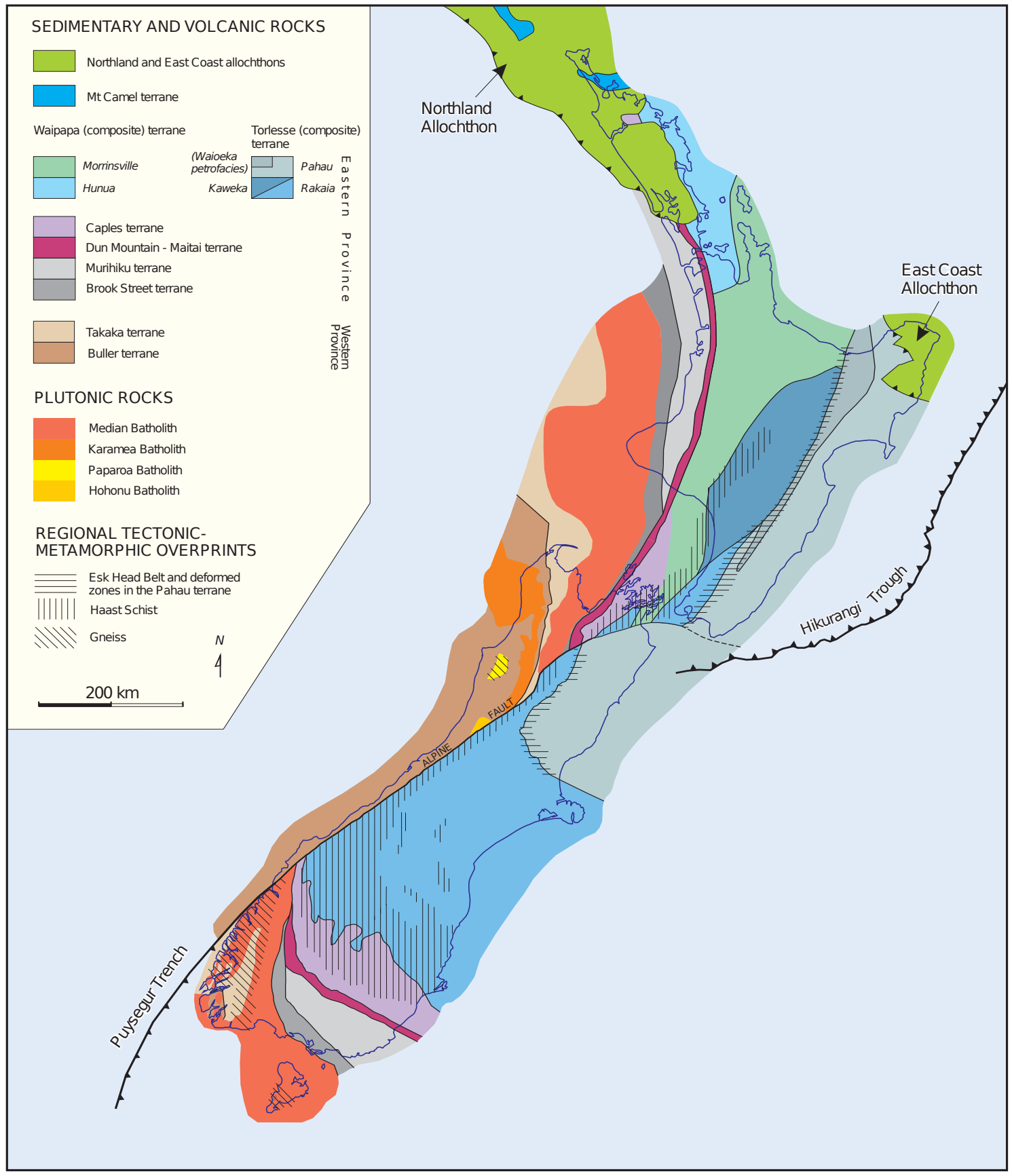

Figure 1.2: New Zealand Basement terranes of (Mortimer, 2004) 


\subsection{Previous ambient seismic noise studies in New Zealand}

In New Zealand the use of ambient noise in seismology is already well established. The availability of a dense national seismograph network, GeoNet (Petersen et al., 2011), as well as detailed prior knowledge of the geological and tectonic environment have allowed testing and development of ambient noise techniques. The first major study into the ambient seismic noise field in New Zealand computed Rayleighwave group velocity maps (Lin et al., 2007) (Figure 1.3). Cross-correlations of year-long vertical component data were used to produce the velocity model. The results showed strong correlations with major geological features, including velocity highs corresponding to the Southern Alps and lower velocities highlighting the Taranaki and Canterbury basins (Lin et al., 2007). Behr (2011) expanded upon these models by processing an enhanced dataset that included several temporary networks throughout New Zealand. The resulting models showed the same features but achieved a higher spatial resolution.

Brooks et al. (2009) characterised the sources of the ambient noise field in New Zealand. The results of a beamforming analysis found two wave phases; the fundamental and first higher mode Rayleigh wave. The presence of the first higher-mode in the noise records was attributed to several possible sources; large wave heights of New Zealand swell combined with deep water approaches to narrow continental margins (Brooks et al., 2009). Behr et al. (2013) also performed beamforming within New Zealand, on a small area within Taranaki, North Island. The vertical and transverse components of the noise field were used to compute noise source directions within the primary and secondary microseism bands. Within the secondary microseism band Rayleigh and Love waves were generated in the same source regions, however differing backazimuths were found for the primary microseism (Behr et al., 2013).

Smaller scale studies were carried out for several locations around North Island. Rayleigh and Love dispersion curves were inverted to obtain 1-D shear-wave velocity profiles for the Northland Peninsula (Behr et al., 2010). The results were in agreement with active source seismic refraction methods used in the same location and a Moho depth of approximately $28 \mathrm{~km}$ was estimated. Behr et al. (2011) used ambient noise to carry out a shear wave tomography study of the Taupo Volcanic Zone. Several 2-D shear wave velocity profiles were computed and the effects of partial melt on the regional velocity field was discussed. Godfrey et al. (2017) studied the surface and shear-wave characteristics of the shallow subsurface beneath the 

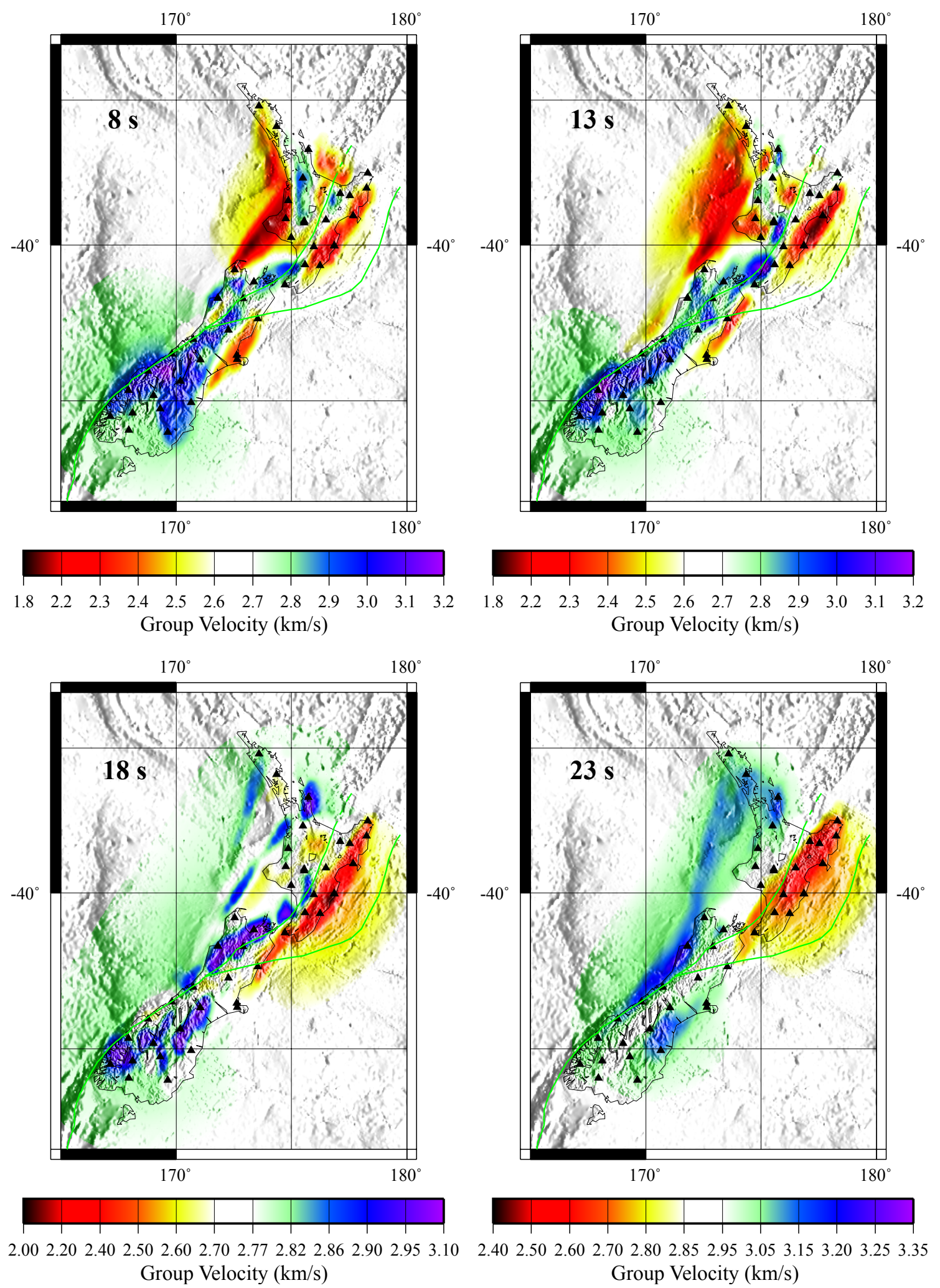

Figure 1.3: New Zealand wide surface wave tomography of Lin et al. (2007). From cross-correlations of vertical component data using stations of the GeoNet national seismometer network (Petersen et al., 2011), Rayleigh-wave group velocity maps were obtained for periods of $8,12,18$ and $23 \mathrm{~s}$. 
Tongariro and Ruapehu volcanoes. Fundamental and first higher-mode dispersion curves for both Rayleigh and Love waves were obtained. Love wave velocities were slower than Rayleigh velocities, in contrast to theoretical dispersion curves, and was attributed to radial anisotropy caused by vertical structures. Azimuthal variations in the differences between Love and Rayleigh dispersion curves further indicated azimuthal anisotropy. Joint inversions of fundamental mode Rayleigh and Love measurements produced shear-velocity models comparable to those obtained using other seismic methods (Godfrey et al., 2017).

In recent years, several ambient noise studies have focussed on the South Island of New Zealand. Fry et al. (2014) used ambient seismic noise recorded on temporary arrays deployed following the 2010 Darfield and 2011 Christchurch earthquakes (e.g. Gledhill et al., 2011; Kaiser et al., 2012) and seismic stations of the permanent national GeoNet network (Petersen et al., 2011) to measure anisotropy from surface wave dispersion of fundamental mode Rayleigh waves throughout Canterbury. At upper crustal depths, east-west trending fast axes parallel to the Greendale Fault and Cretaceous faults were recorded. At lower-crustal depths, fast axes were shown to be parallel to the present plate boundary strain direction (Fry et al., 2014). Ambient noise cross-correlations within the region contain fundamental and first highermode signals at frequencies between 0.1 and $1 \mathrm{~Hz}$ (Savage et al., 2013). For station paths parallel to near-coastal ocean wave directions in the region, comparisons of measured amplitudes of horizontal and vertical cross-correlation showed strong first higher-mode Rayleigh waves. Furthermore, a basement resonance frequency of approximately $0.4 \mathrm{~Hz}$ was obtained from $\mathrm{H} / \mathrm{V}$ ratios of higher-mode Rayleigh waves (Savage et al., 2013).

The MOANA (Marine Observations of Anisotropy Near Aotearoa) ocean-bottom seismometer deployment (e.g. Yang et al., 2012) has been the focus of several ambient noise studies. Yang et al. (2012) characterised the ambient noise recorded offshore New Zealand, identifying the microseism and infragravity peaks. Ball et al. (2016) presented a 3-D shear velocity model for the crust and mantle beneath South Island and the surrounding ocean. Dispersion curves of ambient noise in the $8-25$ s period band were inverted. Low shear velocities of $<4.4 \mathrm{~km} . \mathrm{s}^{-1}$ were modelled beneath the Banks and Otago Peninsulas, with higher velocity mantle anomalies shown beneath the Southern Alps. 


\section{Theoretical background and methodology}

In this chapter a review of ambient seismic noise and its applications are presented. A description of the processing methods used and the theoretical reasoning behind them is given.

Cross-correlations are obtained following the processing framework of Bensen et al. (2007). The schematic flowchart of the major processing steps is illustrated in Figure 2.6 .

\subsection{Ambient noise theory}

\subsubsection{Surface waves and ambient seismic noise}

There are two types of surface waves that propagate through the uppermost crust. Rayleigh (1885) first identified surface waves that are elliptically-polarised shearwaves. The particle motion of Rayleigh waves at the surface is a retrograde ellipse, with a vertical displacement approximately 1.5 times the maximum horizontal displacement, assuming a homogeneous Poisson solid (Stein and Wysession, 2003). This changes at depth with decreasing horizontal amplitudes until motion switches to a prograde ellipse. The apparent velocity of a Rayleigh wave, $L_{r}$ in a half-space is

$$
L_{r} \approx 0.92 \beta
$$

for a Poisson solid, where $\beta$ is the shear-wave velocity (Stein and Wysession, 2003).

Love (1911) further identified surface waves that are linearly polarised in a horizontal plane, called Love waves. Love waves are recorded on transverse component seismograms, and Rayleigh waves are recorded on vertical and radial traces. In general, surface waves dominate seismograms as their amplitudes decay at a rate of $1 / r$, with distance $r$, as opposed to body waves, which decay at a rate of $1 / r^{2}$.

Love (1911) also demonstrated that surface waves are dispersive, meaning propagation velocity varies with frequency and wavelength (Figure 2.1). Seismic velocities 
increase as surface waves' depth of penetration increases. Love waves require a velocity structure that varies with depth, so cannot exist in a half-space, in contrast to Rayleigh waves.

Surface waves recorded by seismograms are the sum of harmonic waves with different frequencies. At a given frequency, a single carrier wave travels at the phase velocity, $c$, given by

$$
c=\omega / k,
$$

where $\omega$ is angular frequency and $k$ is wavenumber. The group velocity, $U$, is the derivative of the phase velocity, and is the envelope of the surface wave packet, given by

$$
U=\frac{\delta \omega}{\delta k}
$$

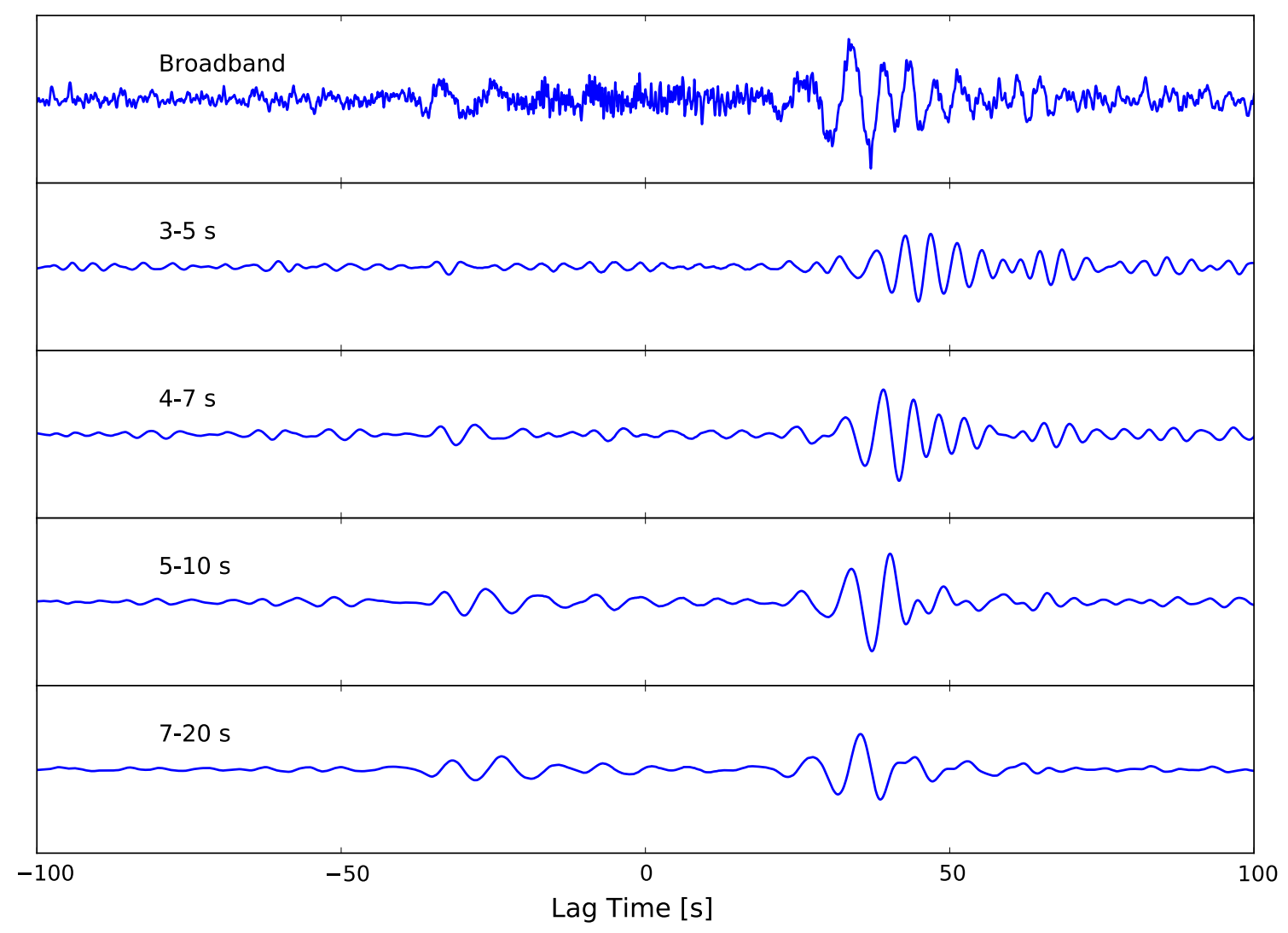

Figure 2.1: Example of surface wave dispersion for station pair BANK1-CCH02. The broadband cross-correlation function (top panel) has been filtered into several period bands; $3-5 \mathrm{~s}, 4-7 \mathrm{~s}, 5-10 \mathrm{~s}$ and $7-20 \mathrm{~s}$ (lower panels, top to bottom). For shorter periods, the surface wave packet can be seen to arrive later, and hence travels slower, than for longer periods.

The partial derivatives of group and phase velocities give their sensitivity to changes in shear velocities, and therefore material properties, at specific periods as a function of depth (Aki and Richards, 2002; Stein and Wysession, 2003). These relationships, often called sensitivity kernels, underpin ambient noise studies that invert surface 
waves for shear velocity models (e.g. Brenguier et al., 2007; Lin et al., 2007; Stehly et al., 2009; Behr et al., 2010). In measuring seismic velocities, we are also inferring information about the intrinsic properties of the material through which the waves travel, such as the density, $\rho$, porosity, $\phi$, the Lamé parameters, $\lambda$ and $\mu$. An indication of the rocks' chemical composition, in addition to any time dependent effects of geological processes, such as earthquakes or volcanic eruptions may also be gained.

\section{Ambient seismic noise}

Seismic noise is typically defined as recorded seismic energy that is not of earthquake origin (Bonnefoy-Claudet et al., 2006). This can be caused by a variety of sources, both anthropogenic, including industrial machinery and vehicles, and environmental, such as the wind or ocean movements, including diurnal tides and fluctuations in air pressure. Gutenberg (1958) produced a list of sources according to frequency and showed that in general noise of high frequencies above $1 \mathrm{~Hz}$ is dominated by noise of anthropogenic origin and lower frequencies of $<1 \mathrm{~Hz}$ have natural origins such as ocean movements. Ambient noise studies in general focus on lower frequencies as they are the most persistent form of seismic noise (Bonnefoy-Claudet et al., 2006). In this frequency band there are two spectral peaks corresponding to oceanic microseisms at $14 \mathrm{~s}$ and $7 \mathrm{~s}$ (Longuet-Higgins, 1950) (Figure 2.2). It has been suggested that the primary microseism at $14 \mathrm{~s}$ is a result of ocean wave movements in near-coastal environments, and their interactions with the sea-floor (Hasselmann, 1963; Friedrich et al., 1998). Longuet-Higgins (1950) suggested that the secondary microseism at $7 \mathrm{~s}$ is generated by non-linear interference of oceanic waves travelling in opposite directions, but with identical frequencies. The dominance of these two microseisms are exploited in seismic noise studies as they are constant and persistent sources of surface waves.

\subsubsection{Cross-correlation technique and seismic velocity retrieval}

The impulse response function, or Green's function, between station pairs can be approximated by cross-correlation of noise records over long time intervals. These cross-correlation functions give information about surface waves (Shapiro and Campillo, 2004). The first insights into the generation of Green's functions from the seismic noise field were obtained by Aki (1957). Aki (1957) measured surface wave velocities from spatial autocorrelations of microtremors. Claerbout (1968) suggested that cross-correlations of noise could be used to produce the impulse response function 

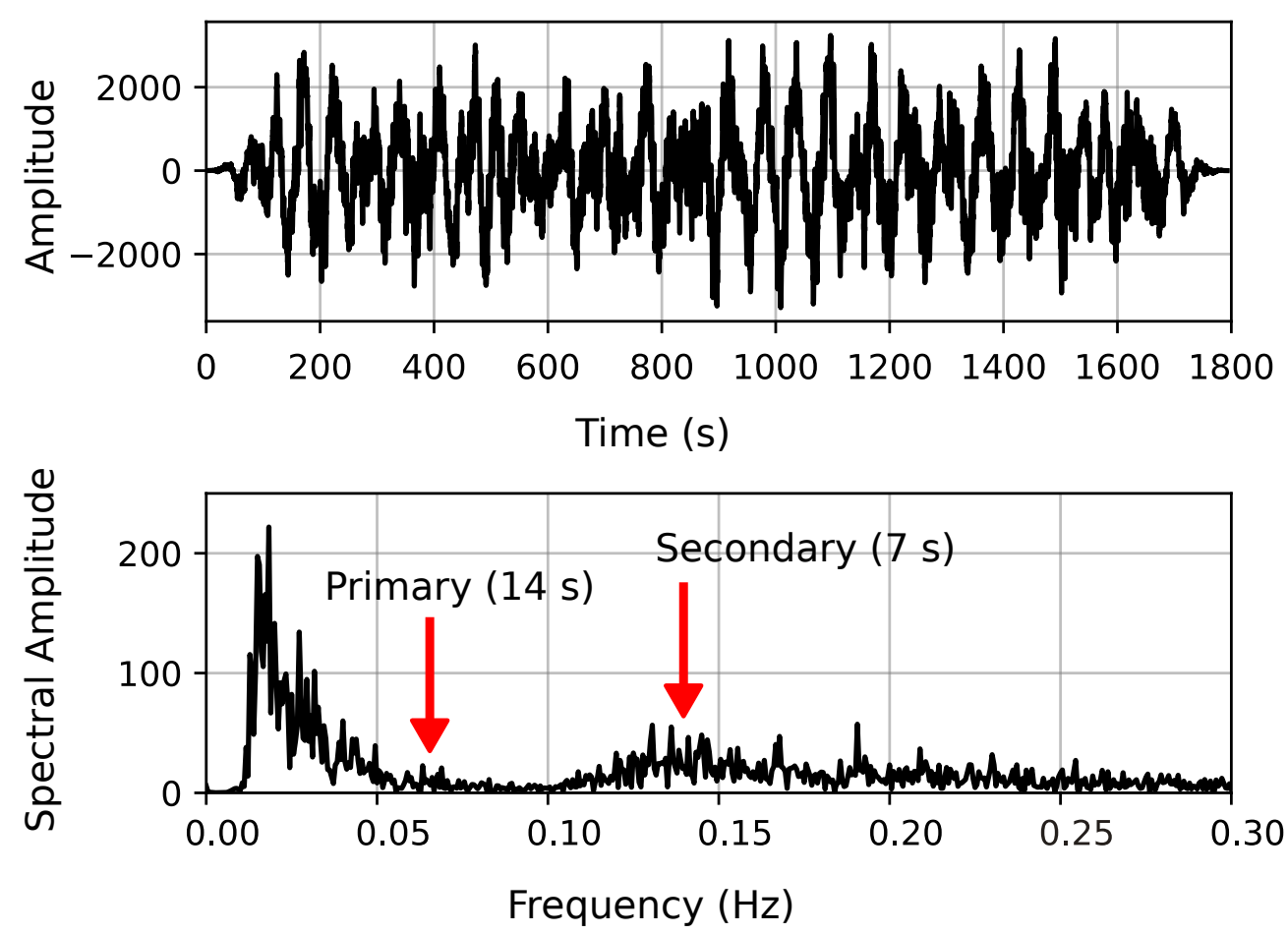

Figure 2.2: Primary and secondary microseisms in continuous seismic recordings. Example presented is for a 30 minute segment of a continuous seismic waveform recorded on GeoNet permanent broadband station CRLZ on 06 November 2010.

of a material. Several decades later, helioseismological studies renewed interest in the application of ambient noise cross-correlations. Duvall et al. (1993) and others (Giles et al., 1997; Rickett and Claerbout, 2000) recovered Green's functions of measured acoustic waves on the Sun's surface, using cross-correlations. Lobkis and Weaver (2001) were able to obtain the Green's function of an aluminium block with cross-correlations of diffuse wavefields of ultrasound noise.

The first applications to seismology were seen soon after, with cross-correlations of earthquake coda produced to give the surface wave component of the Green's tensor (Campillo and Paul, 2003). Shapiro and Campillo (2004) suggested that ambient noise sources randomise over time so Green's functions can be obtained from seismic records of long durations, without the need for large earthquakes. Shapiro and Campillo (2004) measured Rayleigh wave dispersion curves for month-long seismic records. Shapiro et al. (2005) produced surface wave dispersion curves and used the results to compute 2-D tomographic models, which corresponded well to known geological features. Recent studies have since focussed on a wide range of study regions and scales. Yang et al. (2008) computed Rayleigh wave group velocities throughout Europe. In the western U.S.A. ambient noise has been used to produce estimates of regional Green's functions (Sabra et al., 2005), Rayleigh and Love wave maps using cross-components of recorded seismic data (Lin et al., 2008) and surface wave tomographic maps (Shapiro et al., 2005). Initially, most ambient seismic noise 
studies focussed on group velocities. Phase velocities are dependent on the number of wave cycles travelled, unlike group velocities, making them more difficult to measure and so less commonly studied (Ritzwoller and Levshin, 1998). Yao et al. (2006) first measured phase velocities from ambient noise, and this is now common procedure. The results of these studies highlighted major geological features. These results were extended by Lin et al. (2012) to obtain crustal density maps for the near surface from surface wave phase velocities and $\mathrm{H} / \mathrm{V}$ ratios. Density estimates were in excellent agreement with those determined from other methods and were attributed to the study area's known geological features. Stehly et al. (2009) focussed on western Europe to invert computed Rayleigh wave velocities for the depth to the Moho, obtaining a result that was similar to previous calculations. On a smaller scale, a 3-D Shear-wave velocity model was obtained from ambient noise cross-correlations on Piton de La Fournaise volcano (Brenguier et al., 2007).

For a pair of seismic stations, the cross-correlation functions contain positive and negative time lags, which are analogous to the causal and acausal Green's functions (Bensen et al., 2007; Yang et al., 2008). That is to say, positive time lags are the arrival times of waves travelling in one direction as if one station is a point force source and the other the receiver. Negative time lags represent waves travelling in the opposite direction, with roles reversed (Figure 2.1, top panel). The previously mentioned studies and many others have shown this result with both synthetic and real data and given approximations to the Green's functions and retrieval of surface wave velocities.

Theoretical and applied studies have attempted to prove the relationship between Green's functions and ambient noise cross-correlations. Stationary phases were used by Snieder (2004) to produce the Green's function from wavefields recorded at two stations of random sources surrounding the receivers. The stationary phase argument assumes that sources lying near to inter-station paths interfere constructively, and sources away from this line contribute destructively to the cross-correlation functions (Aki and Richards, 2002). Another method demonstrated by Wapenaar (2006) used the reciprocity of the wave equation to reconstruct the Green's function from cross-correlations. Sánchez-Sesma and Campillo (2006) were able to retrieve the Green's function for the realistic cases of unevenly distributed noise sources, assuming a diffuse equipartitioning of seismic wave energy. By cross-correlating traces over long time periods, randomly distributed noise sources average to give improved distribution of noise. Cross-correlation functions are typically dominated by fundamental mode Rayleigh waves. Tsai (2010) explained that the noise sources generating fundamental mode Rayleigh waves are predominantly near-surface, and consequently close to being equipartitioned. 


\subsubsection{Noise directionality across central South Island}

The directionality of signal-to-noise ratios gives an indication of the locations from which ambient noise is originating across the study regions. Prior to producing symmetric cross-correlation functions for dispersion analyses, SNR's of positive and negative lag correlations were computed for each station pair between $0.05-2 \mathrm{~Hz}$ (or $0.5-20 \mathrm{~s}$ ). Figures 2.3 and 2.4 show some results for correlations from several stations. Interstation paths are split in half and coloured according to SNR ratio, with warm colours denoting higher ratios. The recorded waves travel out from the seismic stations, in the direction of the centre of each interstation path. For all stations the SNR's are typically over 10 for noise travelling north and westwards, compared to ratios below 5 for southeasterly surface waves. The dominant source of ambient noise energy in the frequency band of interest is typically oceanic currents and coastal wave interactions. For this region of South Island of New Zealand, Southern Ocean currents dominate recorded ambient noise, as shown in the Southern Alps by higher SNR's for paths travelling northwest. Higher signal-to-noise ratios would be expected for southeasterly travelling waves if near-coastal interactions were the dominant source of ambient noise energy in this region (Figure 2.4).

These findings are in agreement with those of the Lin et al. (2007) study of ambient noise throughout New Zealand. Figure 2.5 shows the results of his SNR directionality analysis, with the length of arrows radiating from stations being proportional to signal-to-noise ratio. For the central Southern Alps and across to the Canterbury Plains, SNR's are highest for cross-correlations of paths travelling northwards. Brooks et al. (2009) additionally found the dominant source of ambient noise energy originating from the south of New Zealand, caused by strong Southern Ocean currents. Such a strong directionality of noise sources throughout the South Island could cause bias in velocity models from ambient seismic noise, as the noise field is clearly not equipartitioned. Cross-correlation functions are expected to be highly asymmetric for NW-SE trending interstation paths. Higher amplitude surface waves will likely be recorded on correlations for signals travelling in a northwesterly direction. To overcome this problem, symmetric cross-correlation functions will be calculated (Section 2.4.1).

\subsubsection{Temporal velocity variations}

When measuring seismic velocities we are also inferring information about the intrinsic properties of the material the waves travel through, in addition to any effects of geological processes, such as earthquakes or volcanic eruptions. Using traditional 

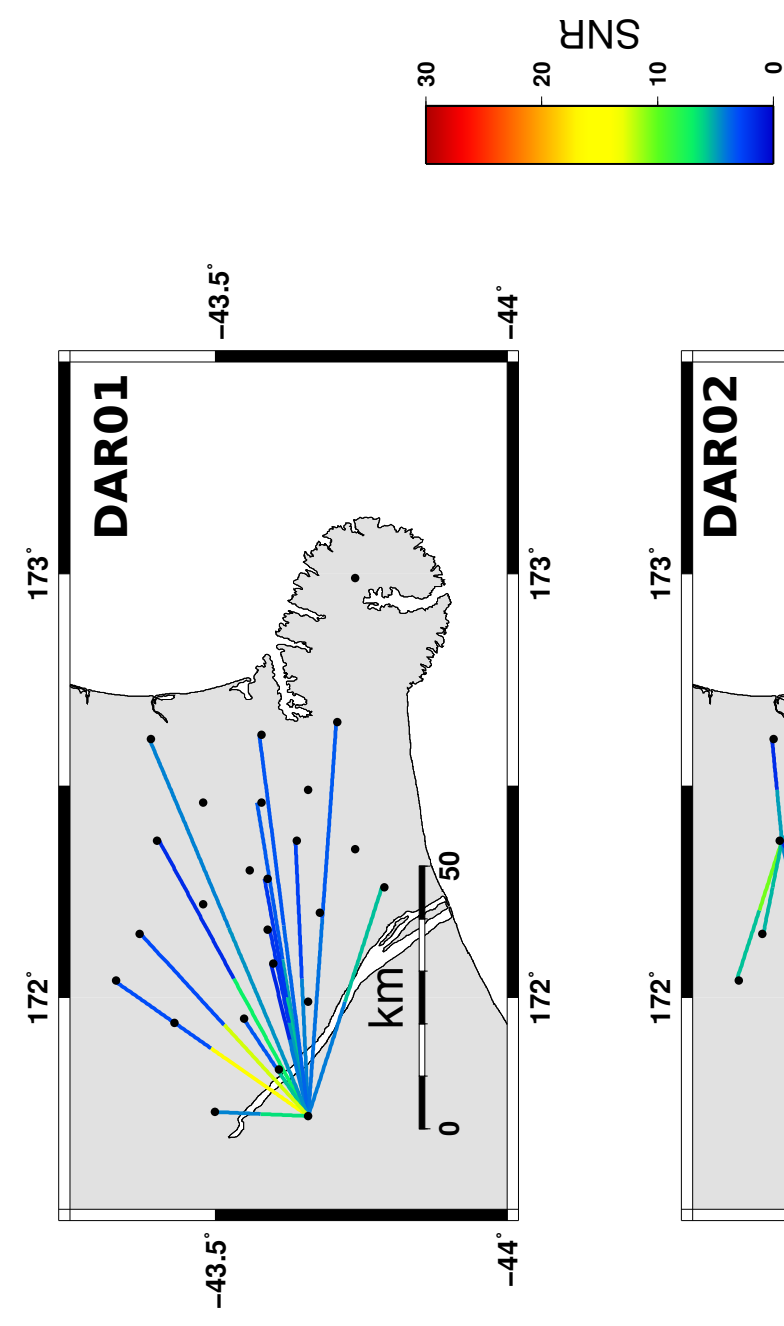

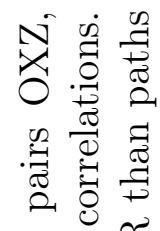

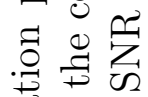

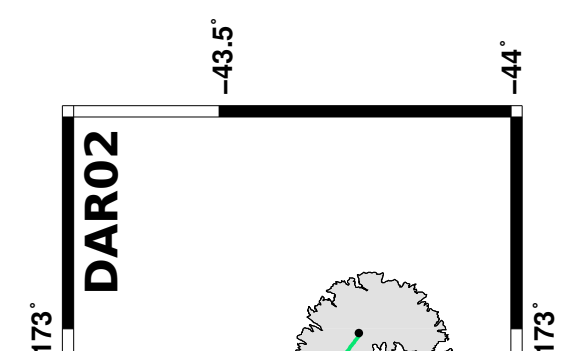

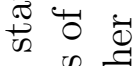

돌.

$\beta$ 苑 $\sigma$

武

恋

규워

占

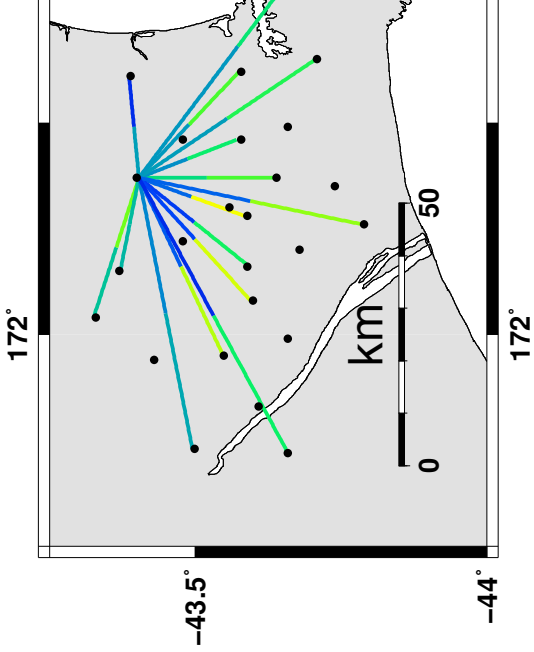

记

苟

$>$ Ð

菊

.01 0

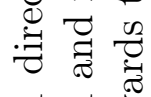

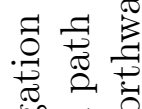

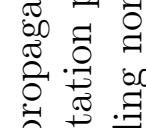
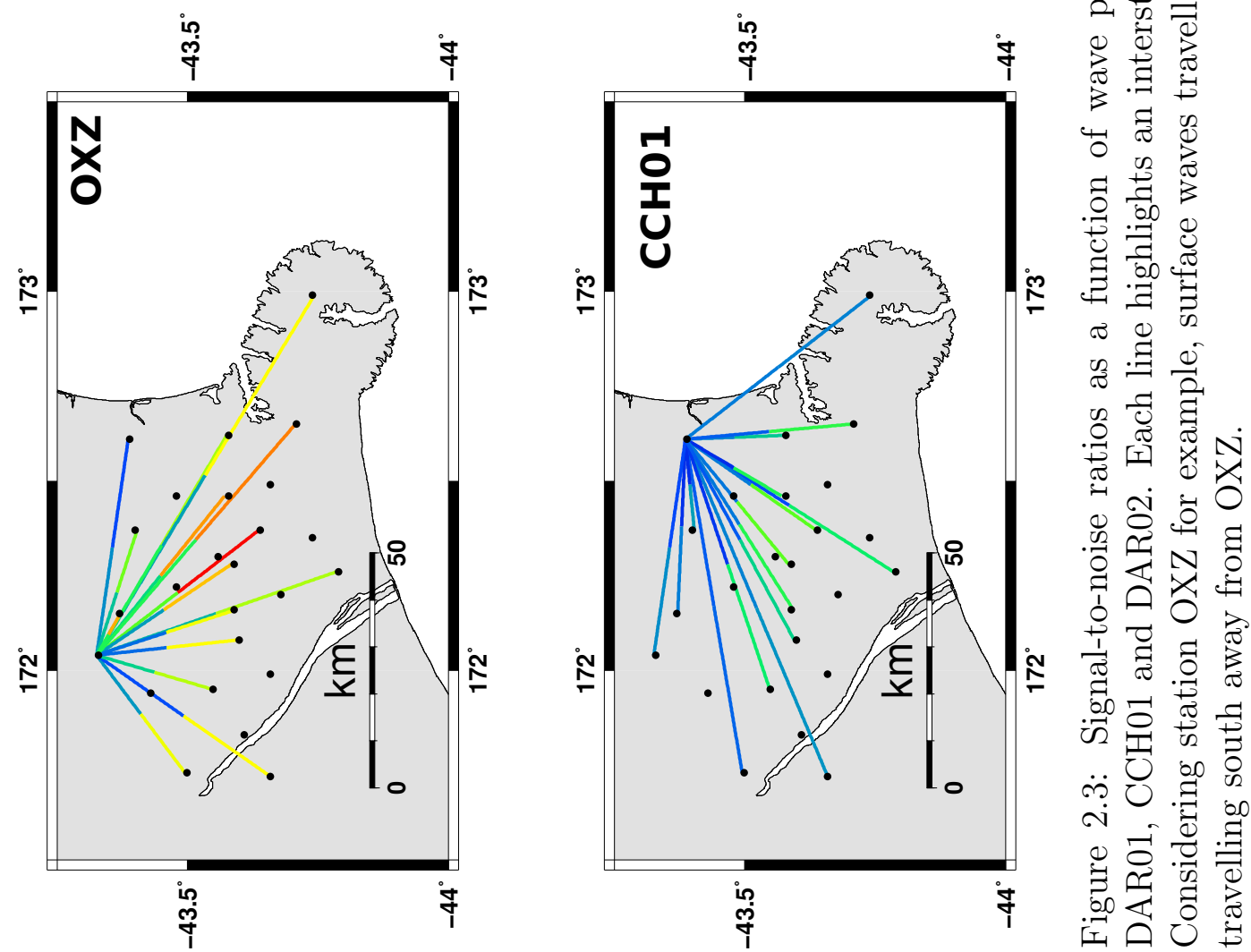


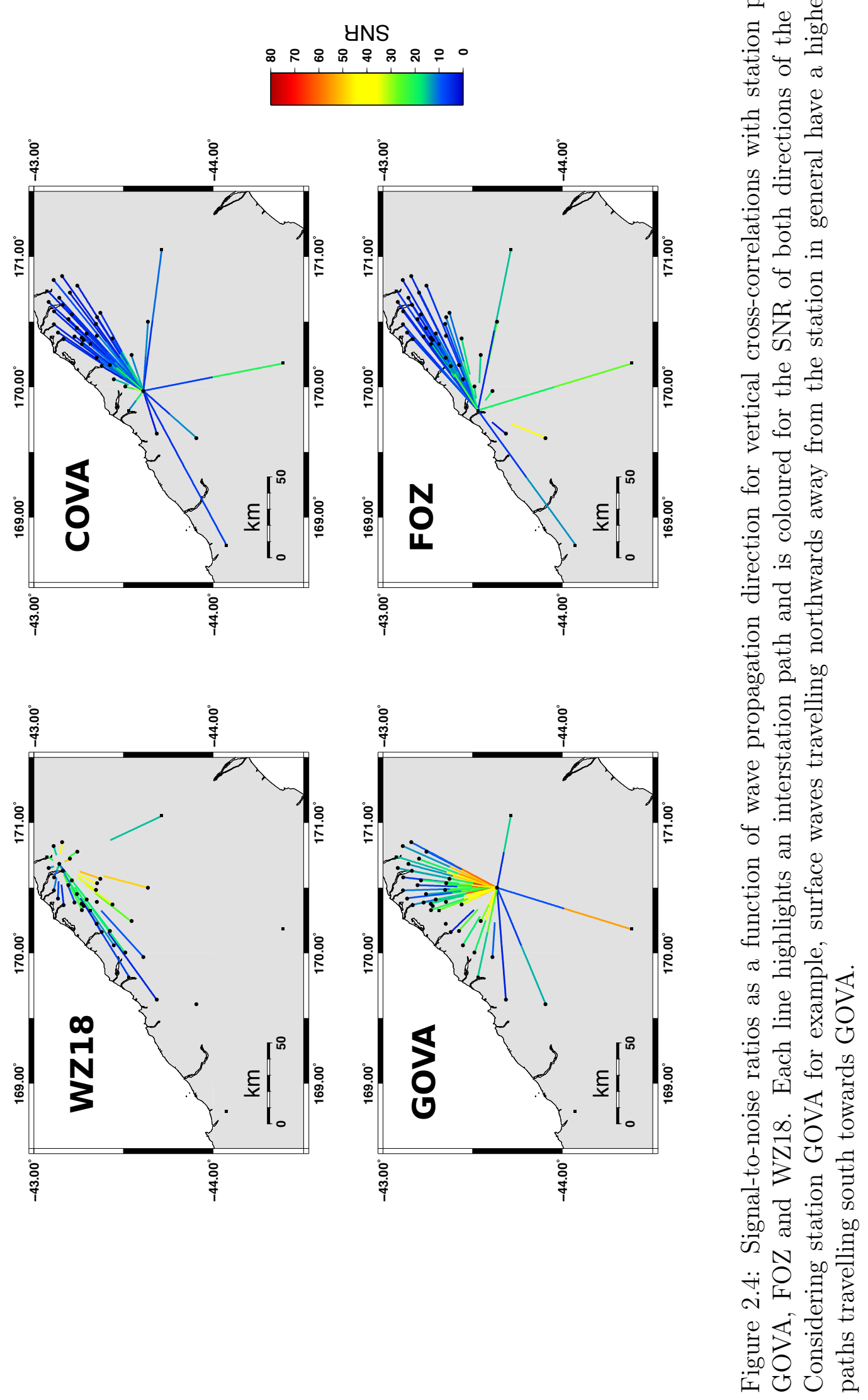




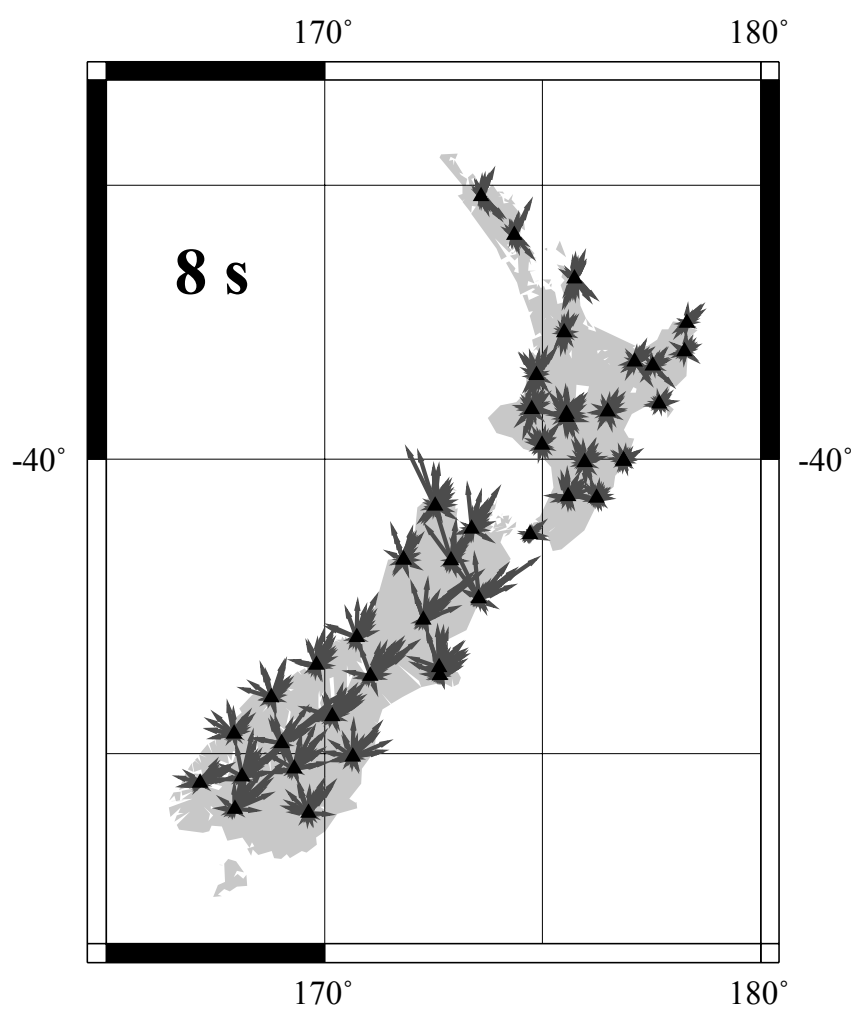

Figure 2.5: Noise directionality at New Zealand GeoNet seismic stations. Arrows propagating from each station in the direction have lengths proportional to the SNR recorded on cross-correlations from the seismic station, normalised by path length. From Lin et al. (2007) 
seismological methods, $\mathrm{P}-\mathrm{S}$ wave traveltimes, $\mathrm{V}_{P} / \mathrm{V}_{S}$ ratios and anisotropy have been investigated to investigate crustal changes. These methods however can be subject to errors with earthquake locations and origin times (Clarke et al., 2011). Poupinet et al. (1984) introduced a new method for computing changes in seismic wave travel times using earthquake doublets from cross-correlations. Following the 1979 M5.9 Coyote Lake earthquake, California, shear-wave velocity decreases of as much as $0.2 \%$ were observed. Several explanations have been suggested for temporal velocity changes following earthquakes, including post-seismic relaxation and tectonic stress changes (e.g. Poupinet et al., 1984; Brenguier et al., 2008).

The technique of Poupinet et al. (1984) was extended for ambient seismic noise and successfully used to detect seismic velocity variations (Brenguier et al., 2008; Wegler et al., 2009). A method was formally established by Ratdomopurbo and Poupinet (1995) and is known as Moving-Window Cross-Spectral (MWCS) analysis. They demonstrated its application using earthquake doublets, with the assumption that the similarity between the two recorded waveforms is an indication of the proximity of sources to one another. It then followed that the distance between the two sources was far smaller than the source-receiver distance. They state that the detection of temporal changes in the velocities of coda waves between two doublet families, a family being a set of co-located earthquakes, indicates changes in physical properties of the sampled medium (Ratdomopurbo and Poupinet, 1995). Sens-Schönfelder and Wegler (2006) further explored the technique of passive image interferometry, identifying advantages of ambient noise over earthquake doublets. Firstly, ambient noise can be recorded continuously. Secondly, there will always be some differences in earthquake source mechanisms and locations, unlike scattered waves of ambient seismic noise. This allows for better recovery of the Green's functions, even in the coda (Wegler and Sens-Schönfelder, 2007).

Relative delay times between long duration 'reference' stacks and shorter duration 'moving' stacks of cross-correlation functions are computed. If a seismic wave is perturbed, the arrival time of the moving stack will differ from the reference. Snieder et al. (2002) showed that this difference, $d t$, is proportional to the arrival time of the reference wave, $t$, and the velocity change, $d v$,

$$
d t=-\frac{d v}{v} t
$$

The relative velocity change for the time period of a particular moving stack is then the negative of the relative arrival time change. Brenguier et al. (2008) implemented this technique on data collected on stations near the San Andreas Fault, following the 2004 Parkfield earthquake. Immediately following the event a velocity decrease 
of $0.08 \%$ was observed, and took over three years to return to pre-Parkfield levels. Time-dependent changes in ambient noise fields have also been exploited to show seasonal variations due to atmospheric pressure changes (e.g. Hillers et al., 2015) or to monitor landslides (e.g. Mainsant et al., 2012). Rivet et al. (2014) recorded velocity changes associated with slow slip events in 2008 in Mexico by using an adapted version of this method, and MWCS has been applied to lunar seismic recordings to show thermal expansion of soil from solar heating (Sens-Schönfelder and Larose, 2008).

\section{$2.2 \quad$ Pre-processing}

Pre-processing of the raw data includes several important steps required prior to cross-correlation (Figure 2.6, Step 1). The aim of these initial processing operations is to enhance ambient noise signals and suppress transient signals such as earthquakes, to maximise the surface wave energy for estimated Green's functions. Removal of any gaps in recordings and instrumental errors is also crucial. It is desirable to approximate as closely as possible a noise source distribution that is spatially and temporally unbiased (Roux, 2009, Section 2.1.2). Pre-processing removes any contamination that results in a non-diffuse wavefield that isn't spatially uniform (Yao et al., 2006). Firstly raw continuous waveform data is downsampled, then the mean, trend and instrument responses are removed to be consistent across different networks (Section 2.2.1). A broad bandpass filter is applied and traces are cut to day-long files. Frequency normalisation and time-domain normalisation are applied (Sections 2.2.2 and 2.2.3). In both normalisations only amplitude information is altered, leaving phase data unchanged. They are both non-linear processes (Bensen et al., 2007) and so the order of operations is important. Finally, before computation of the cross-correlation functions, horizontal component waveforms are rotated from North and East orientations to radial and transverse components. As described in Section 5.2.2, for some Southern Alps seismic stations where seismometers are not oriented to standard horizontal directions, commonly borehole instruments, waveforms are first rotated to true North and East, before rotating to the radial and transverse orientations.

The parameters used for pre-processing steps have to be carefully considered as they are heavily dependent on the data (e.g. waveforms with high or low numbers of recorded earthquakes) and desired target. Bandpass filters and spectral normalisation filters depend upon the limits of the recording sensors, the period band of interest and the spatial distribution of seismic stations (Poli et al., 2012). For both regions considered in this study, waveforms with periods under $1 \mathrm{~s}$ are disregarded. 
The maximum period depends on the inter-station path, as described in the Quality control and error analysis section of this chapter.

Glitches that may be caused by instrumentation and recording issues are removed, to avoid contaminating cross-correlation functions with false phases. To do this the Seismic Analysis Code processing system, or SAC, (Goldstein and Snoke, 2005) is used. Next, a symmetric cosine taper of $1 \%$ is applied to both ends of each daily waveform, to prevent the occurrence of spectral leakage due to discontinuities between the initial and final samples of a given trace (Gubbins, 2004).

\subsubsection{Instrument response removal and polarities}

For structural studies using ambient seismic noise, when cross-correlating seismic records recorded on several different sensors the amplitude and phase responses of each instrument must be removed from the data. These responses of seismometers are dependent upon wave frequency, and vary for different sensors. The relationship between these responses and the true ground motion is defined by the frequency response function (e.g. Scherbaum, 2013). The amplitude response of seismic sensors is greatest above the instruments' natural frequency, giving a flat response curve (Figure 2.7). Broadband instruments output a wide frequency band within which the response is flat, whereas short-period sensors have a high natural frequency, often approximately $1 \mathrm{~Hz}$, thereby giving a much smaller bandwidth within which the amplitude response is flat. The phase response of instruments is due to phase delays between ground motion occurring and the induced voltage being recorded (Havskov and Alguacil, 2004). These effects are demonstrated in Figure 2.7 which give the response curves for representative intermediate-period and broadband seismometers (red and blue curves respectively).

To remove instrument responses, the TRANSFER function of SAC (Goldstein and Snoke, 2005) was performed on all daily traces. This function removes the instrument response through deconvolution and applies a secondary instrument response by convolution within a certain frequency band, to give consistency across all instrument types considered. A wide bandpass filter is applied to prevent long-period noise being excessively amplified. For example, for raw data with a sampling frequency of $100 \mathrm{~Hz}$, a filter of $0.01-50 \mathrm{~Hz}$ is applied. Responses in RESP format are removed using the EVALRESP option of the TRANSFER function.

If polarity information has been included in the response files, any incorrect polarities may be corrected at this stage. This is examined visually after response removal and any remaining inconsistencies are dealt with. When the instrument response 


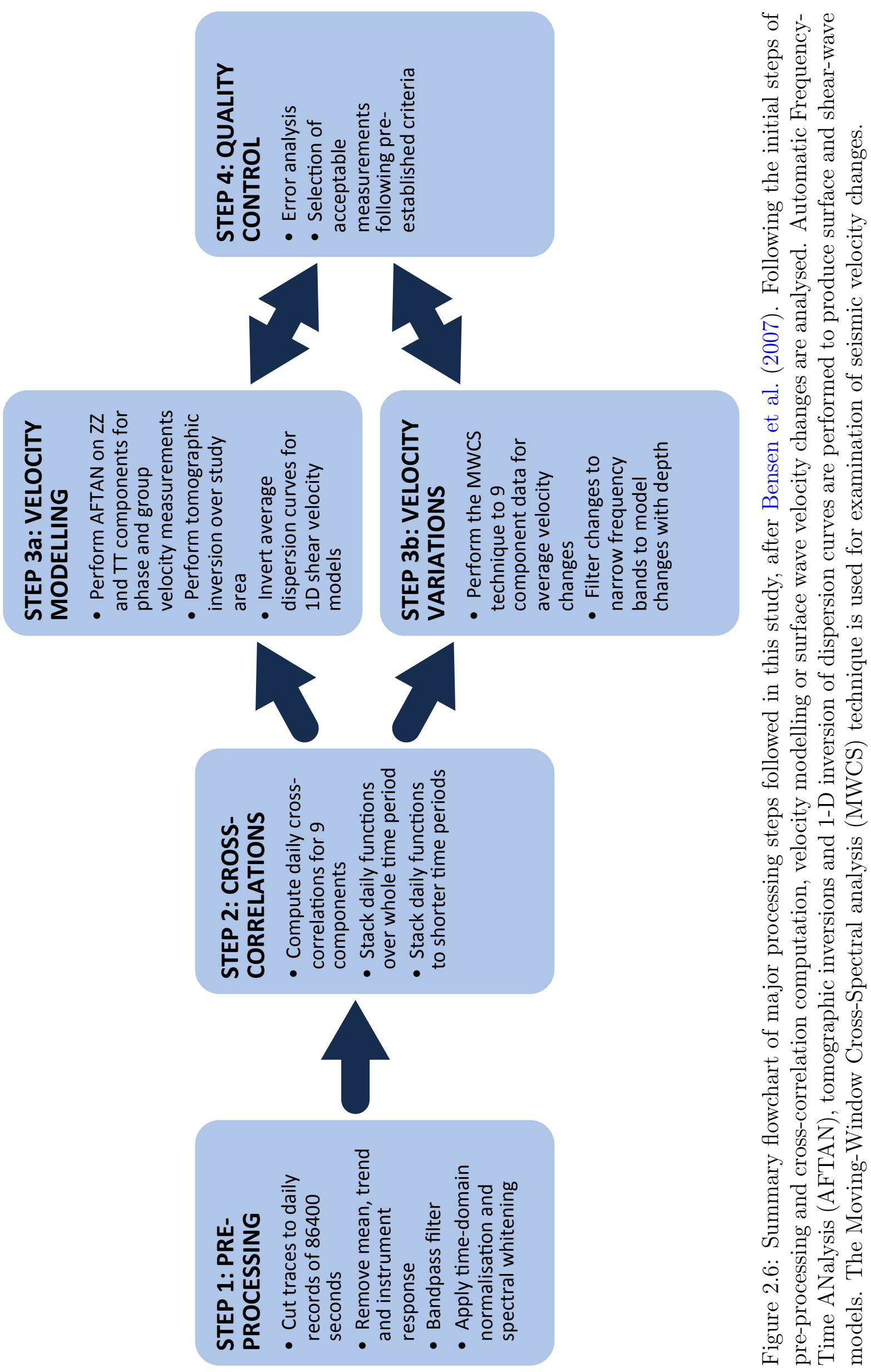




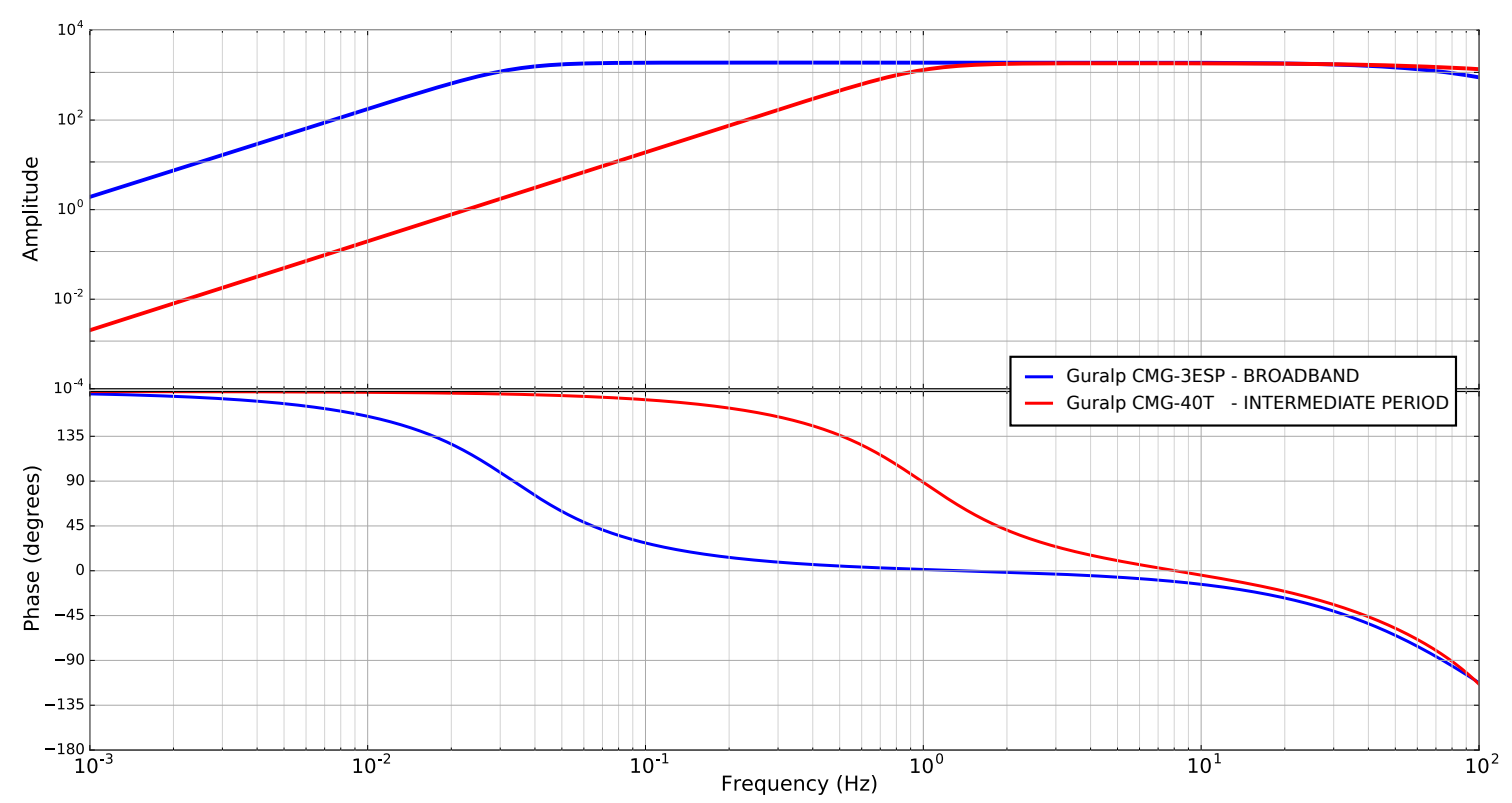

Figure 2.7: Amplitude and phase response curves for Guralp sensors. The responses for CMG-3ESP broadband sensors are given in blue and the CMG-40T intermediate period sensor is shown in red.

correction has been applied, first arrivals of teleseismic earthquake signals are examined. There should be no visible difference in the earthquake coda before and after removal for broadband stations. The polarities of each component are also checked to be consistent across dataset in the same way.

\subsubsection{Spectral whitening}

Frequency domain normalisation through spectral whitening suppresses monochromatic signals and enhances lower amplitude signals (Bensen et al., 2007). This includes the primary and secondary microseism bands, which are present at approximately $7 \mathrm{~s}$ and $14 \mathrm{~s}$ (Longuet-Higgins, 1950). Whitening is achieved by inversely weighting the amplitude of the signal's spectrum by a smoothed version of itself within a specified frequency band (Bensen et al., 2007; Stehly et al., 2009). The resulting signal has a smoothed and more equally distributed, flatter frequency spectrum. Figure 2.8 shows an example for a 30 minute segment of raw data, before and after whitening below $2 \mathrm{~Hz}$ and one-bit normalisation.

\subsubsection{Time-domain normalisation}

Time domain normalisation is performed on each of the day-long traces. This step suppresses earthquake signals and enhances amplitudes of scattered waves. It also removes instrumental contamination from any remaining glitches. There are several 
methods that can be used to carry out time-domain normalisation and the choice of which to use is largely dependent on the dataset.

Root-mean-square (RMS) clipping reduces the dominance of earthquake signals in the resultant cross-correlations by clipping amplitudes to a multiple of the signals' RMS or standard deviation for a given day (e.g. Sabra et al., 2005; Roux, 2009). This approach is not suitable for the post-Darfield data, as smaller amplitude aftershock signals may not be removed if a larger event is present, which increases the daily RMS amplitude.

One-bit normalisation is the most aggressive temporal normalisation method (Bensen et al., 2007); whereby all positive amplitudes are given a value of 1 and negative amplitudes are replaced by -1 , given by,

$$
\hat{x}_{i}=\left\{\begin{array}{crrr}
1 & & x_{i}>0 \\
0 & \text { for } & x_{i}=0 \\
-1 & & x_{i}<0
\end{array}\right.
$$

Although it is the most aggressive, one-bit normalisation is the most commonly used method of temporal normalisation in ambient noise studies (e.g. Brenguier et al., 2007; Mordret et al., 2013; Savage et al., 2013).

A third method that is commonly employed is automated event detection and removal. Where amplitudes are above a critical threshold, a window about the event is set to zero. The selection of the threshold will vary between stations and the zero-function window width is subjective. A disadvantage of this technique is that where high levels of seismicity are present, this technique may remove large chunks of the waveform if the thresholds are set too low, or not sufficiently remove unwanted signals if set too high (Bensen et al., 2007).

Behr et al. (2011) and Lin et al. (2007) implemented a running-absolute-mean normalisation. Instrument response removed data are bandpass filtered and smoothed for the frequency range in which earthquake surface waves are expected. The original waveform is then inversely weighted with the smoothed filtered waveform. This process is robust in removing earthquake signals, but is not always appropriate for traces with instrumental glitches, as these are not removed.

Although some studies choose not to perform time-domain normalisation (e.g. Stehly et al., 2009), for datasets containing high seismicity levels it is an extremely important pre-processing step. This is the case for the post-Darfield deployments used in this study, where aftershocks with a wide range of magnitudes dominate. Earthquake signals that are not removed by time-domain normalisation can appear in the resultant cross-correlations as spurious high amplitude arrivals, close to the origin 

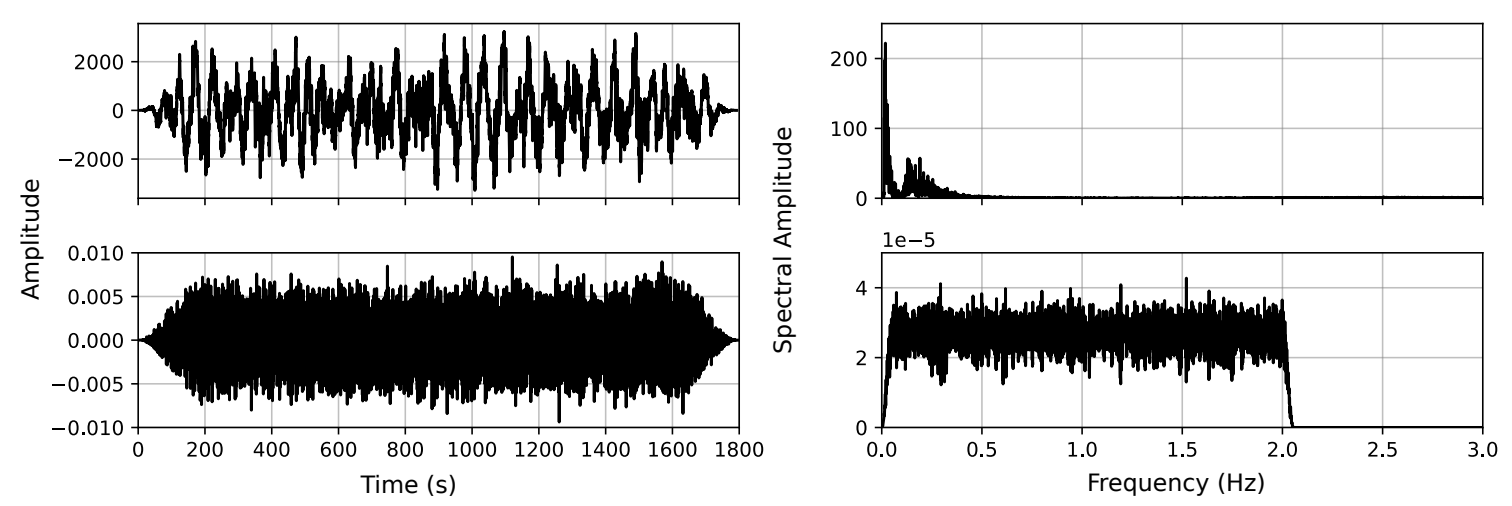

Figure 2.8: Effect of time and frequency domain normalisation on raw data, prior to cross-correlation processing. Example presented is for a 30 minute segment of a continuous seismic waveform recorded on GeoNet permanent broadband station CRLZ on 06 November 2010. The upper panels show the raw data time series and frequency spectrum, with the lower panels showing the same data after the application of the normalisations.

time that dominate the function. In this study one-bit normalisation is employed due to its robustness in removing aftershock signals.

\subsection{Cross-correlation computation}

Following pre-processing, daily traces are cross-correlated (Figure 2.6, Step 2). For each station pair, where data are recorded for both stations on a given day, each component of one is cross-correlated with each of those of the second station. This gives nine functions; ZZ, ZR, ZT, RR, RT, RZ, TT, TR, TZ, where Z, R and $T$ denote vertical, radial and transverse respectively. This notation will be used henceforth. The resulting signals have both positive and negative time lags. For positive time lags, the first letter implies a signal propagating from the first station in that direction, and the second letter is the component on which it was recorded at the second station. Notation is vice versa for negative time lags. The signals are cross-correlated in short time windows and stacked to produce daily cross-correlation functions.

The daily functions are then stacked in several ways. All available daily crosscorrelations are stacked to give a 'reference' function for each station pair. Using the maximum number of functions possible converges the signal and gives the closest approximation to the Green's function. The assumption is made that there are no considerable changes in the cross-correlations over the period of study. Crosscorrelations for each day are examined to look for any visibly bad functions. Examples of what is considered bad may be very low signal-to-noise ratios or correlations 
with main surface wave arrivals at times that differ greatly from other days. Any such correlations are removed from subsequent processing. For the examination of temporal seismic velocity variations in Canterbury shorter 'moving' stacks are calculated (discussed further in Chapter 4).

\section{$2.4 \quad$ Velocity modelling}

\subsubsection{Surface wave dispersion}

Dispersion analysis is performed on symmetric functions, produced from the stacked cross-correlation functions (Figure 2.6, Step 3a). Cross-correlations are split into negative and positive signals about $0 \mathrm{~s}$ lag-time. The negative signal is reversed and added to the positive function. Symmetric cross-correlations improve the signal-tonoise ratio (SNR) as coherent surface wave signals will add together and increase in amplitude, whilst incoherent noise interferes destructively.

Dziewonski et al. (1969) defined the multiple-filter technique for the determination of group velocities by applying many narrow bandpass filters to a Fourier transformed signal. This study uses automatic frequency-time analysis, or AFTAN, which is a modified version of this method (Levshin et al., 1972, 1992; Levshin and Ritzwoller, 2001), to compute phase and group velocities for Rayleigh and Love waves. Polarisation of surface waves using ambient seismic noise can been determined (Roueff et al., 2009). Here, the assumption is made that Rayleigh waves are polarised to the $\mathrm{RR}$ and ZZ correlation components and Love waves are visible on TT components. Several studies have shown that this is an acceptable approximation (e.g. Lin et al., 2007; Stehly et al., 2009; Savage et al., 2013). The cross-components of vertical and radial correlations (i.e. RZ, ZR) also contain Rayleigh wave information, including higher modes.

Group velocities are calculated from the measured arrival times of the waveform for each bandpassed frequency band. The Gaussian bandpass filters, $H(\omega)$, are defined as,

$$
H_{n}(\omega)=e^{-\alpha k}
$$

where

$$
k=\left(\frac{\omega-\omega_{n}}{\omega_{n}}\right)^{2}
$$

after Dziewonski et al. (1969), with $\alpha$ as the parameter controlling resolution and filter width and it is selected according to interstation distance, $d$ (Levshin et al., 1989). The group arrival time, $t_{\max }$ is defined as the envelope maximum at a central 
angular frequency, $\omega_{n}$, for the $n$th filter. The group velocity is then approximated as,

$$
U\left(\omega_{n}\right)=\frac{d}{t_{\max }} .
$$

Spectral leakage can occur if the spectrum of the waveform is not flat (Bensen et al., 2007), resulting in a central frequency of the narrow filtered traces that does not accurately represent its frequency content. To correct for this phenomenon, the corresponding instantaneous frequency is computed for each group velocity measurement, to replace the central frequency. The instantaneous frequency is the time derivative of phase, $\Phi$, of the filtered signal, given by

$$
\omega=\left|\frac{d \Phi}{d t}\right|_{t_{\max }},
$$

(Bracewell, 1978; Bensen et al., 2007).

The phase velocity, $c$, at the group travel time $t_{\max }$ is therefore defined by Lin et al. (2008) as

$$
c=\frac{\omega}{k}=\frac{r \omega}{\Phi\left(t_{\max }\right)+\omega t_{\max }-\frac{\pi}{4}-2 \pi N},
$$

where $\omega$ is angular frequency, $k$ is wave-number, $r$ is the interstation distance and $\Phi$ is the phase of the filtered cross-correlation function. A phase ambiguity in the measurement is defined by $2 \pi N$, where $\mathrm{N}$ is the number of complete cycles at a frequency of $\omega$.

A priori knowledge of the subsurface structure and expected dispersion curves is required to determine the correct number of cycles and prevent 'cycle-skipping' (Lin et al., 2008). For this purpose reference dispersion curves from synthetic seismograms of average regional velocity models are used. The constant $\frac{\pi}{4}$ is required in equation 2.10 due to the influence of noise sources not lying on the great-circle path connecting the seismic stations (Harmon et al., 2007). To compute phase and velocity dispersion measurements, the codes of Levshin and Ritzwoller (2001) are used in this study.

\subsubsection{Surface wave tomography}

Surface wave dispersion curves are inverted to create 2-D regional velocity maps at discrete periods. The method employed throughout this study follows that of Barmin et al. (2001) that is based heavily on minimising a 'penalty function' through the application of regularisation or damping parameters. These parameters include a spatial smoothing function and constraints on the amplitude of the velocity pertur- 
bation from a reference model (Barmin et al., 2001). For $n$ dispersion measurements and a grid of $m$ cells, the penalty function is given as,

$$
(\mathbf{G m}-\mathbf{d})^{T} \mathbf{C}^{-1}(\mathbf{G m}-\mathbf{d})+\mathbf{m}^{T} \mathbf{Q m},
$$

where $\mathbf{m}$ is the model vector of velocity perturbations from the reference model, $\mathbf{d}$ is the input data vector of observed travel-time residuals with respect to the reference model, $\mathbf{C}$ is the covariance matrix of observational errors, $\epsilon_{i} . \mathbf{G}$ is a matrix of linear functionals, $G_{i}$, that link the observed traveltime residuals to the model, such that for $i=1, \ldots, n$,

$$
d_{i}=G_{i}(\mathbf{m})+\epsilon_{i}
$$

$\mathrm{Q}$ is a $k \times k$ regularisation matrix, defined by,

$$
\mathbf{Q}=\mathbf{F}^{T} \mathbf{F}+\mathbf{H}^{T} \mathbf{H}
$$

The matrix $\mathbf{F}$ includes the spatial smoothing constraints, $\alpha$ and $\sigma$ :

$$
F_{j j^{\prime}}=\left\{\begin{array}{cl}
1 & j=j^{\prime} \\
-\alpha S\left(\mathbf{r}_{j}, \mathbf{r}_{j^{\prime}}\right) / p_{k} & j \neq j^{\prime}
\end{array}, \quad p_{k}=\sum_{j^{\prime}} S\left(\mathbf{r}_{j}, \mathbf{r}_{j^{\prime}}\right)\right.
$$

where $\mathbf{r}_{j}$ and $\mathbf{r}_{j}^{\prime}$ are location vectors of cells $j$ and $j^{\prime}$ and,

$$
S\left(\mathbf{r}_{j}, \mathbf{r}_{j^{\prime}}\right)=K \exp \left(-\frac{\left|\mathbf{r}_{j}-\mathbf{r}_{j^{\prime}}\right|^{2}}{2 \sigma^{2}}\right)
$$

(Behr, 2011). $\mathbf{H}$ is a penalty function, that weights for path density in a given cell $j$

$$
H_{j j^{\prime}}=\left\{\begin{array}{cl}
\beta \exp \left(-\rho\left(\mathbf{r}_{j}\right)\right) & j=j^{\prime} \\
0 & j \neq j^{\prime}
\end{array},\right.
$$

where $\beta$ is a damping parameter and $\rho\left(\mathbf{r}_{j}\right)$ is the path density (Barmin et al., 2001).

The weighting parameters $\alpha$ and $\sigma$ are chosen by systematically varying the parameters for a range of combinations to find the minimal mean misfit, as in Behr (2011). It was found that this was achieved with $\alpha=200$ and $\sigma=25$ for a cell spacing of $0.1^{\circ}$. Barmin et al. (2001) suggests using $\beta=1$ and this is implemented in this study. 


\subsubsection{Shear wave inversion}

Following 2-D tomographic inversions of both Rayleigh (R) and Love (L) waves, for group $(G)$ and phase (c) velocities, 1-D inversions for shear velocity are computed. The picked dispersion curves are 'path-averaged', meaning that they represent average velocities for the entire interstation path. Using these measurements in problematic in shear-wave inversions as resultant 1-D models cannot be located at a single, discrete location. Additionally, if there are any small velocity anomalies anywhere along the interstation path, these can bias the dispersion measurements. A way to overcome this issue in shear-wave inversions is to produce dispersion curves from the tomographic models. At a given location within the tomography grid, the dispersion measurements are interpolated to form new curves which are used as input for the shear-wave inversion. Using dispersion measurements obtained from the tomographic inversions removes some effects of velocity path-averaging, as it relies on the assumption that several cross-correlation paths have passed through each cell point, and measurements have previously been weighted accordingly throughout the tomography process.

The Neighbourhood Algorithm (NA) of Sambridge (1999a,b) is employed for the highly non-linear process of shear velocity inversion. The Dinver software package is used (Project, 2017), after Wathelet et al. (2008).

The NA is a stochastic search method that searches the whole parameter space in order to minimise a misfit function. Pseudo-random samples are generated within the parameter space and forward modelled for dispersion curves (Wathelet et al., 2004). The method uses Voronoi cells, and samples the parameter space by dividing it into cells of irregular sizes and shapes. Each cell is the nearest neighbour of a previous sample and the search method steers progressive inversions away from local minima of the misfit in order to fully sample the entire model space.

A priori models are given as ranges of the shear-velocity, density and layer thickness. The data misfit of each forward model to the input dispersion curve is given as

$$
\text { misfit }=\sqrt{\sum_{i=0}^{n_{F}} \frac{\left(x_{d i}-x_{c i}\right)^{2}}{\sigma_{i}^{2} n_{F}}}
$$

where for each point, $i$, in the dispersion curve, $x$ and $f$ are velocity and frequency, with subscripts $d i$ and $c i$ denoting the input data and calculated curve. $\sigma$ is the uncertainty in the input velocity and $n_{F}$ defines the number of points in the the curve (Wathelet et al., 2004). The inversion is run over a defined number of iterations and the weighted mean of all models with misfits below a defined threshold is calculated, 
to find a preferred shear-velocity model. Throughout this study the threshold was set to a misfit of 0.05 , which is a $5 \%$ difference between the modelled and input dispersion curves.

\subsection{Velocity variations: Moving-window cross-spectral analysis}

Relative velocity variations are computed by comparing reference and moving stacks using the MWCS analysis method of Poupinet et al. (1984). The methodology described here is the scheme developed by Clarke et al. (2011). There are two main steps to the technique, firstly the computation of relative time delays, and secondly the calculation of relative velocity variations and error analysis. It is assumed that the seismic wave speed is constant along the interstation path, i.e. it is travelling through a homogeneous medium. There are several important input parameters that must be chosen carefully considering both the input signal and target structure. The lengths of the reference and moving stacks depend on the amplitude and duration of expected velocity changes. The length and shift of moving windows are dependent on the frequency content and length of the cross-correlation functions. Further discussion on the selection of processing parameters is given in Chapter 4, Section 4.3 .

The computation of delay time, $d t$, between the reference and moving stacks, is performed on the cross-correlations in the spectral domain using a series of overlapping time windows. Each pair of reference and moving stacks is divided into short time windows, of days to months, and a delay time is measured (see Chapter 4, Section 4.3 and Figure 4.3). They are transformed into the spectral domain, given by

$$
X(\nu)=F_{r e f}(\nu) \cdot F_{m o v}^{*}(\nu)
$$

where $X(\nu)$ is the cross-spectrum of the Fourier transforms of both time series (Clarke et al., 2011). The asterisk denotes that the complex conjugate of $F_{\text {mov }}(\nu)$ is used. The cross-spectrum can be represented as the product of its amplitude, $|X(\nu)|$, and phase, $\phi(\nu)$,

$$
X(\nu)=|X(\nu)| e^{i \phi(\nu)}
$$

To achieve correct delay time measurements it is required that the short windowed reference and moving stacks are sufficiently similar (Clarke et al., 2011). The coherences between the Fourier transformed times series are computed in each short window to give values between 0 and 1 . A high coherence is desired and a threshold 
must be set, below which delay time measurements are disregarded. For Canterbury a coherence of 0.7 or higher was required (see Section 4.3). From the cross-spectrum, the delay time is given in the phase spectrum by,

$$
\phi=m \cdot \nu, \quad m=2 \pi \delta t_{i}
$$

The time delay, $\delta t_{i}$, for the $i$ th window, and its uncertainty, can be estimated from the slope of a linear regression of frequency versus phase (see example in Figure 4.3). The delay times are proportional to the arrival time of the reference function $t$ and the velocity change $\delta v$ (Poupinet et al., 1984),

$$
\delta t=-\frac{\delta v}{v} t
$$

Thus, to obtain relative velocity variation measurements, the inverse slope of a linear regression of the delay time measurements with arrival, or correlation lag time, is calculated for each pair of reference and moving stacks.

\subsection{Quality control and error analysis}

Several quality control measures were implemented throughout the processing procedure. For velocity measurements in dispersion analyses, only station pairs with a minimum interstation distance of two wavelengths were considered, to ensure that several full surface-wave cycles were completed between stations (Lin et al., 2007). If a simplified speed $=$ distance $\times$ time relationship is assumed, the cut-off period, $\tau_{\max }$, is given by,

$$
\tau_{\max }=\frac{\Delta}{2 U},
$$

for a given interstation distance $\Delta$ and Rayleigh-wave group velocity, $U$, after Bensen et al. (2007). Considering an interstation distance of $50 \mathrm{~km}$ and $U=1.5 \mathrm{~km} . \mathrm{s}^{-1}$ for example, this gives a $\tau_{\max }$ of $16.7 \mathrm{~s}$. There is no consensus as to the minimum number of wavelengths that should be used. Three wavelengths is suggested as an appropriate value by Bensen et al. (2007), whereas (Brenguier et al., 2007) stated that only one wavelength can still give meaningful dispersion measurements.

Secondly, the signal-to-noise ratio (SNR) of the cross-correlations is calculated. For a given correlation function, the waveform is split corresponding to the 'signal' segment and the 'noise' segment (Figure 2.9). The signal is determined from the inter-station distance and includes the surface wave velocities that are to be expected for the region. The noise portion of the trace is considered to be the waveform that arrives before and after the signal as scattering (Lin et al., 2007). Amplitudes are 


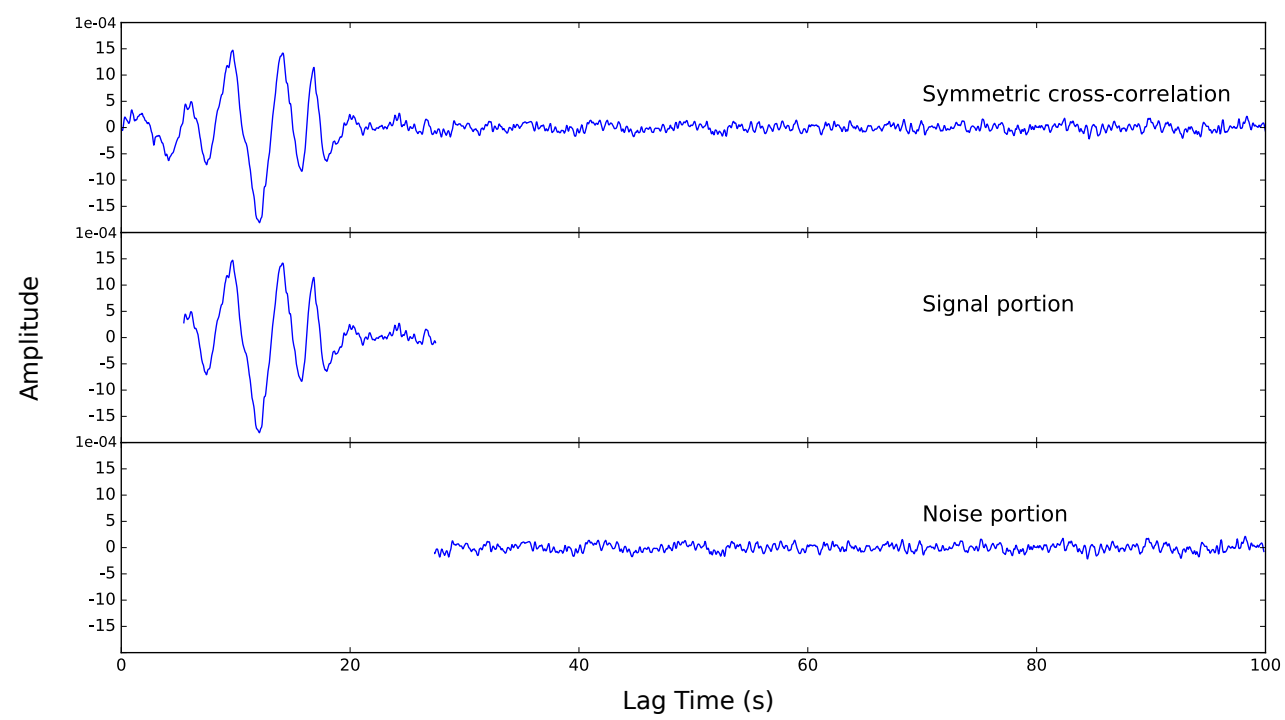

Figure 2.9: Schematic examples of signal-to-noise calculation. The symmetric crosscorrelation (top panel) is split into the signal (centre panel) which is dependent on expected surface wave velocities and a noise portion (lower panel), which is the waveform for all time lags after the surface wave packet. The example shown is the stacked vertical component cross-correlation for Canterbury station pair MQZBANK1.

made positive and the maximum absolute amplitude of the signal is divided by the standard deviation of the noise segment, to give a singular SNR value per crosscorrelation function. Cross-correlations below a SNR threshold are rejected from both dispersion and velocity variation processing, and the threshold is subjective to the dataset considered and chosen after a thorough visual inspection of the data, so as to retain the highest quality measurements without drastically reducing the dataset. Signal to noise ratios are computed over the whole bandwidth of the given signal and for smaller period bands so that the ratio can be considered as a function of period.

Synthetic Green's functions are generated for the study regions, using a regional velocity model, for Love and Rayleigh waves (Herrmann and Ammon, 2004). Fundamental and first-higher modes are considered for ZZ, ZR, RZ, RR and TT components, assuming a point source and interstation distances corresponding to those considered for each study region. These functions are then run through dispersion analysis to provide a control for picking modes in the processed data.

The dispersion picking software (Levshin and Ritzwoller, 2001) does not provide an assessment of uncertainties in the code. To ascertain uncertainties, in addition to producing dispersion curves for the long-duration reference stacks, shorter 30-day stacks are examined for variability in the surface wave dispersion. 
Finally, when computing MWCS delay times, in order to only examine the surface wave, cross-correlation lag times that give surface wave velocities outside of the expected range for the region are discarded. This range of lag times is dependent on inter-station distance and again assuming a speed $=$ distance $\times$ time relationship. 


\section{Surface wave and shear velocity models of Canterbury, New Zealand}

\subsection{Introduction}

An introduction to the Canterbury region has been presented in Chapter 4, Section 4.2. Mortimer (2004) presents the basement rock of the region as the Torlessee Composite Terrane, comprised of greywacke and argilite. The basement is Permian to Triassic in age (Browne et al., 2012). The overlying sedimentary cover comprises repeating successions of sandstone, mudstones and limestone, in a thin layer $1-2$ km thick, Cretaceous to Cenozoic in age (Forsyth et al., 2008). At Banks Peninsula, the basement is interbedded with flows of Cretaceous andesite and rhyolite (Sewell et al., 1992). The dominant faulting regime in the region is Late Cretaceous normal faulting, with predominantly E-W strikes. These faults were formed when subduction halted along the Hikurangi Margin in the mid-Cretaceous, and was followed by a period of extension and volcanism (Davy et al., 2008). Several of these Cretaceous faults were reactivated in the recent Canterbury Earthquake Sequence (Sibson et al., 2011), including the Greendale Fault of the $2010 \mathrm{M}_{w} 7.1$ Darfield event (Ghisetti and Sibson, 2012).

Following the recent Canterbury earthquakes, several studies have examined the seismic structure of the region. Reyners et al. (2014) created tomography models of $\mathrm{V}_{P}$ with a velocity high underneath Banks Peninsula, inferred as a basaltic plug at $10-15 \mathrm{~km}$ depth. Models of $\mathrm{V}_{P} / \mathrm{V}_{S}$ ratios showed low ratios of $<1.6$ surrounding the Greendale Fault, and up to $15 \mathrm{~km}$ north of the fault's surface trace (Reyners et al., 2014), determined from tomography of earthquake arrival times. (Syracuse et al., 2013) created a 3D model of the region from $\mathrm{P}$ and $\mathrm{S}$ arrival times of relocated aftershocks. The resulting $\mathrm{V}_{S}$ models show relative uniformity, with an increase in velocity from approximately $2 \mathrm{~km} . \mathrm{s}^{-1}$ at the surface to $3.3-3.6 \mathrm{~km} . \mathrm{s}^{-1}$ at $14 \mathrm{~km}$ depth. (Syracuse et al., 2013) found elevated velocities in the shallow subsurface in the northwest of the study area, attributed to thinning sedimentary cover at the foothills of the Southern Alps. Similarly, high velocities at $2 \mathrm{~km}$ depth up to 3.3 
$\mathrm{km} . \mathrm{s}^{-1}$ were modelled and interpreted as the volcanics of Banks Peninsula (Syracuse et al., 2013).

This study uses several stations of the national seismic network, in addition to local temporary networks. A major advantage of using temporary seismic deployments in addition to the national permanent network is an enhanced path coverage across the region. It is also demonstrated that meaningful cross-correlations and subsequent velocity models can be obtained with less than ideal deployment lengths and network configurations.

\subsection{Data}

Following the Darfield earthquake of September 2010, several rapid-response networks were deployed, with the aim of recording the aftershock sequence (Figure 3.1). The New Zealand national natural hazard monitoring organisation, GeoNet, deployed nine short-period sensors, which were operational from 5 September to 29 September 2010 (Gledhill et al., 2011). A combination of 13 broadband and shortperiod sensors were deployed in a joint effort by Victoria University of Wellington (VUW), The University of Wisconsin-Madison (UWM) and The University of Auckland (UA) (e.g. Syracuse et al., 2012; Savage et al., 2013), herein denoted as the VUW-UWM-UA deployment. The stations were placed throughout the Canterbury region and were in operation for 4 months from 18 September 2010 to 13 January 2011. Data from these two temporary networks were processed for crosscorrelations separately as they cover different time periods and each collection of data was enhanced with the nearby permanent broadband stations, CRLZ, MQZ, OXZ and RPZ, of the national GeoNet (Petersen et al., 2011) seismometer network. The complete catalogue of cross-correlations was used for dispersion analyses and inversions. The arrays consisted of seismic stations lying on and surrounding the Greendale Fault, with inter-station paths ranging from $6 \mathrm{~km}$ to $156 \mathrm{~km}$ (Figure 3.1). Further information about each deployment, including sensor types is given in Appendix A. Instrument response files were not available for the VUW-UWM-UA stations, and so had to be created prior to processing. Despite both deployments being out for approximately one and four months respectively, not all stations were operational at all times, as is often the case with temporary seismometer deployments. Stations either had issues with equipment or site conditions. A schematic of the data availability of all stations is shown in Figure 3.2. 


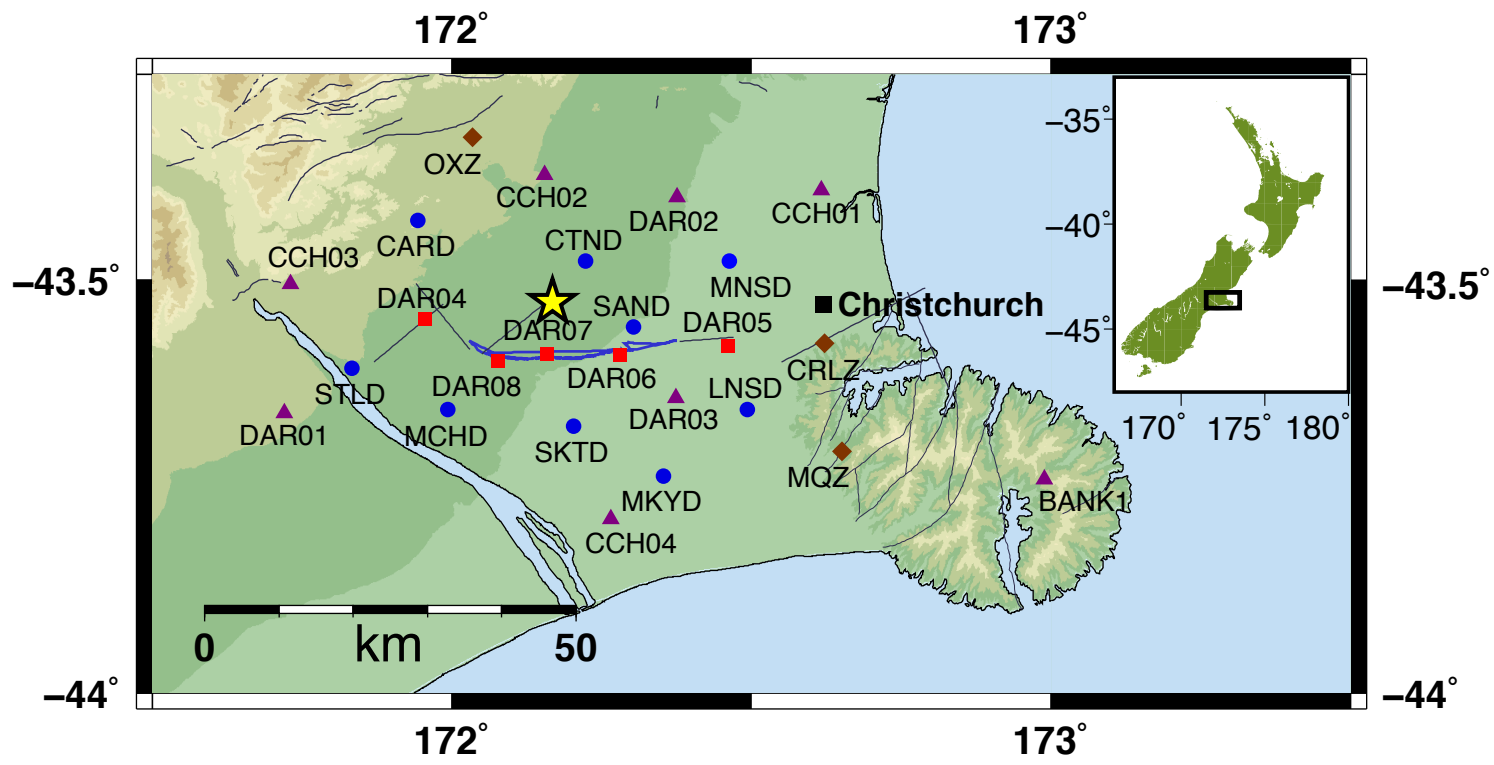

Figure 3.1: Map of Canterbury area showing the epicentre of the Darfield earthquake (yellow star). Mapped faults are shown by thin grey lines and the Greendale Fault Quigley et al. (2012) is highlighted in blue. Coloured symbols show locations of GeoNet permanent broadband stations (brown diamonds), GNS Science temporary stations (blue circles), and VUW-UWM-UA (red squares and purple triangles for on- and off-fault stations, respectively). Inset shows the location of the study area within New Zealand.

\subsubsection{Data preparation}

The VUW-UWM-UA continuous seismic waveform data were converted from hourly miniseed files to day-long records of $86400 \mathrm{~s}$. All GeoNet data were downloaded in daily files using the Continuous Waveform Buffer client (GeoNet, 2016).

For processing efficiency and to enhance surface wave signals, daily waveforms were split into shorter segments (in this study fifteen minutes is used), cross-correlated and stacked for each day. Often seismologists will create continuous waveforms by padding any gaps with zeroes. This causes problems with cross-correlation computation however, since if the same time segment for any two given stations has been zero-padded, they correlate perfectly with a coherence of 1, creating erroneous spikes at zero-offset in the resultant correlation function. In this study, any short fifteen minute segment that contains gaps for either of the stations being correlated was thrown out from subsequent processing. Waveforms were also checked for glitches or unexpected high amplitude spikes, which were removed if present, by removing bad points and linearly interpolating between data on either side.

The raw data for each station are then prepared following the procedure outlined in Section 2.2. The mean and trend are subtracted from each daily trace, a cosine taper of $1 \%$ is applied to each end of the record and the instrument response is 


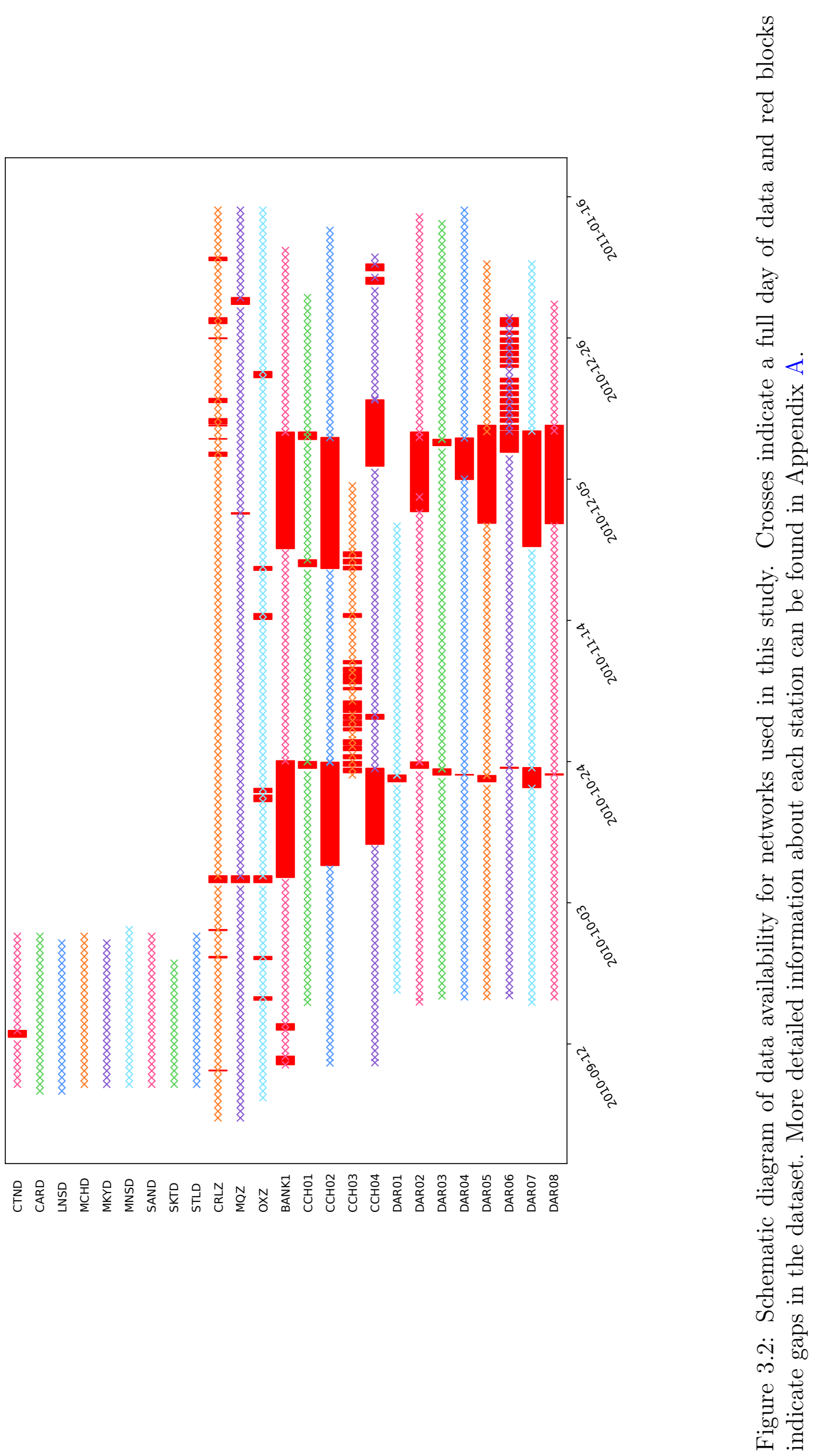


Table 3.1: Cross-correlation processing parameters used for the Canterbury study.

\begin{tabular}{|l|l|}
\hline Sampling frequency & $25 \mathrm{~Hz}$ \\
\hline Pre-processing bandpass filter & $0.01-12 \mathrm{~Hz}$ \\
\hline Time-domain normalisation & one-bit applied \\
\hline Whitening filter & $0.05-2 \mathrm{~Hz}$ \\
\hline Cross-correlation segment length & 15 minutes \\
\hline Maximum correlation time lag & 100 seconds \\
\hline SNR threshold & 4 \\
\hline
\end{tabular}

removed.

\subsection{Methods}

\subsubsection{Cross-correlation computation}

Stacked cross-correlation functions were computed using the methods explained in Section 2.3 and the MSNoise processing package (Lecocq et al., 2014). Table 3.1 gives a summary of the parameters used for all steps to obtain the cross-correlation functions. The day long vertical and horizontal component data were first enhanced using the preprocessing steps of resampling to $25 \mathrm{~Hz}$ to be consistent across networks, bandpass filtering to 0.01 to $12 \mathrm{~Hz}$. In addition, frequency- and time-domain normalisations were applied, as spectral whitening between 0.05 and $2 \mathrm{~Hz}$ and onebit normalisation. For each station pair the horizontal components were rotated to radial $(\mathrm{R})$ and transverse $(\mathrm{T})$ orientations and all components were cross-correlated in fifteen minute windows, for computational speed and efficiency. These are then stacked to produce daily correlation functions, which are in turn stacked for all available days to give a single cross-correlogram for each of the nine Green's function tensor components for each station pair. The lengths of the final stacked functions range from 18 to 151 days. For this study the ZZ, RR and TT components are used for velocity modelling. The remaining cross-components are used in the temporal variation study presented in Chapter 4.

Figure 3.3 shows the effect of stacking over all available cross-correlation functions for station pair DAR04-DAR07. Stacking over long durations suppresses incoherent noise and improves the SNR of the surface wave signals. The stability of the crosscorrelation functions through time can be examined, to identify days with spurious arrivals or inconsistent features. When cross-correlations are stacked over multiple days, incoherent signals are suppressed if they are have sufficiently low amplitudes or do not correlate coherently with signals at the same times on other days. It is still 
important however to look at daily functions in addition to stacks to understand and categorise signals present in the correlation functions.

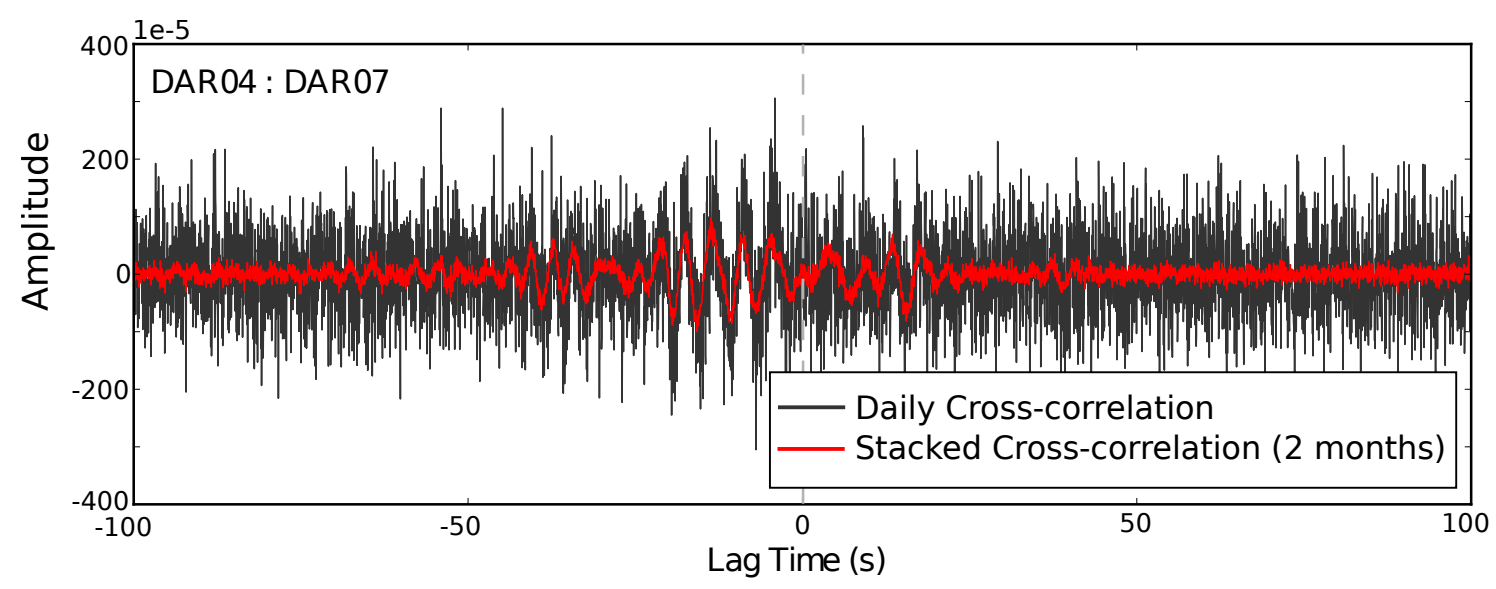

Figure 3.3: Effect of stacking on cross-correlation functions. The example shown is the vertical component cross-correlation function for station pair DAR04-DAR07 (For station locations see Figure 3.1). The black trace is an individual daily correlation for 19 November 2010 and the red line is the corresponding average stack. This example is the same as presented in Figure 4.1, lower panel.

Figures 3.4 and 3.5 show examples of daily RR cross-correlations for two station pairs; MQZ-DAR06 and DAR02-DAR04 alongside their corresponding stacked correlation functions (top panels). The station path of MQZ-DAR06 strikes NW from approximately $20 \mathrm{~km}$ south of Christchurch up to the eastern extent of the Greendale Fault's surface rupture (Figure 3.1 for station locations). A coherent surface wave arrival is present at a lag time of $12 \mathrm{~s}$ each day. There is a lower amplitude arrival at the same time in the negative portion of the Green's function. The station path DAR02-DAR04 strikes WSW, ending at the westernmost extent of the Greendale Fault. The dominant surface wave arrival for this pair is travelling NE and is visible at $-18 \mathrm{~s}$ in the daily correlation functions (Figure 3.5). The interstation distances for these two paths are $33.2 \mathrm{~km}$ for MQZ-DAR06 and $37.8 \mathrm{~km}$ for DAR02-DAR04. Any functions that do not follow the overall trends visible on other days are removed if signal amplitudes are higher than the background ambient noise levels. Figure 3.5 shows such a daily correlation function on 23 October 2010. The surface wave arrivals are not seen for this day and there are higher amplitudes of background noise compared to other days.

Prior to FTAN processing, the symmetric functions are created for each stacked cross-correlation (see Section 2.4.1). Figure 3.6 gives the cross-correlation functions for the vertical and horizontal components as a function of interstation distance, in the $0.05-2 \mathrm{~Hz}$ frequency band. Surface-wave move-out velocities are between 1 $3 \mathrm{~km} . \mathrm{s}^{-1}$ across the network for the $\mathrm{ZZ}$ component. The RR correlations show a 


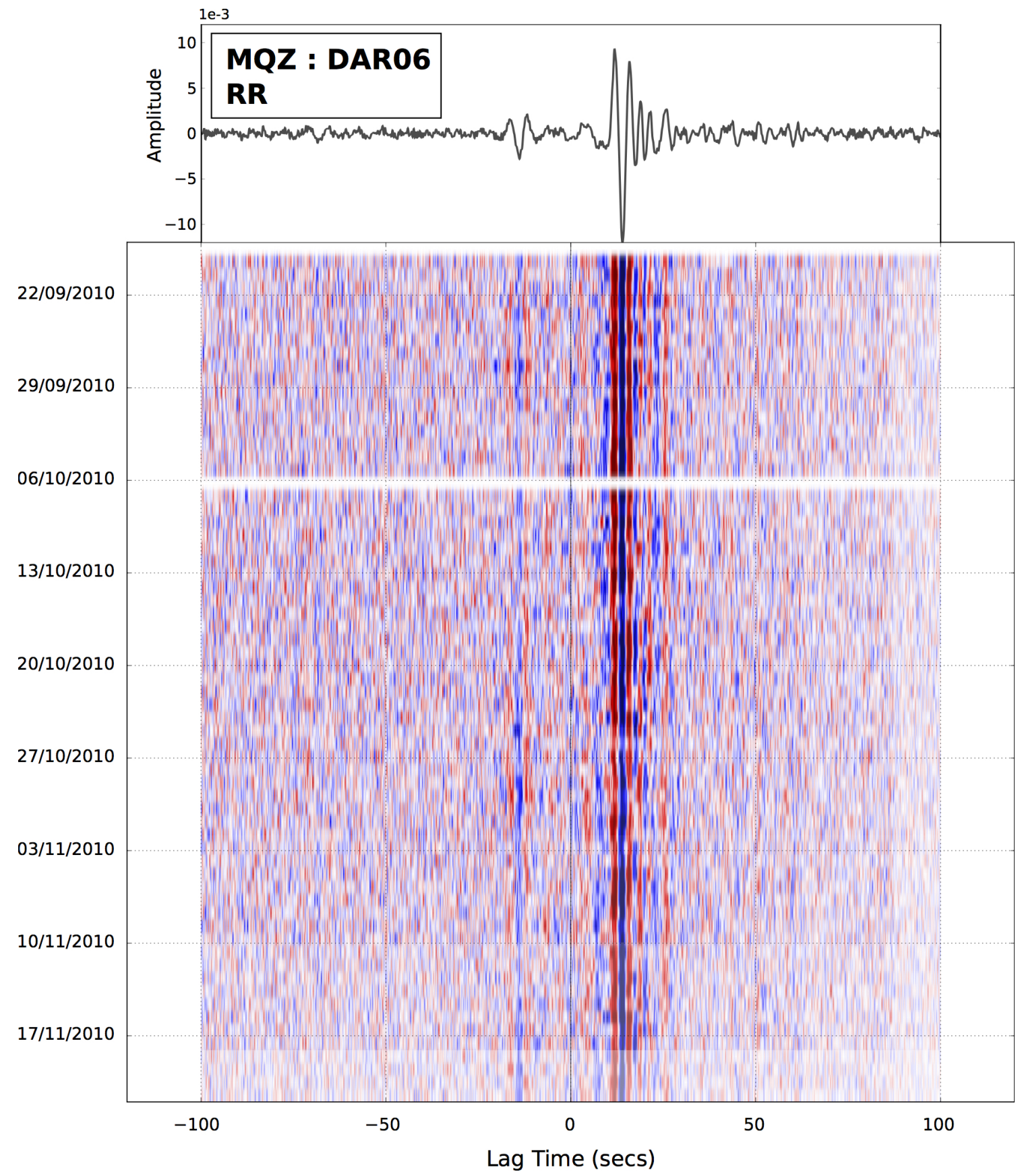

Figure 3.4: Daily RR correlation functions of station pair MQZ-DAR06 (see Fig. 3.1 for station locations), for a 2 month period from September 20th until November 20th 2010. The functions are normalised to the highest amplitude over the time period shown, with colour intensity proportional to amplitude. The top panel gives the corresponding stacked reference function for comparison. 


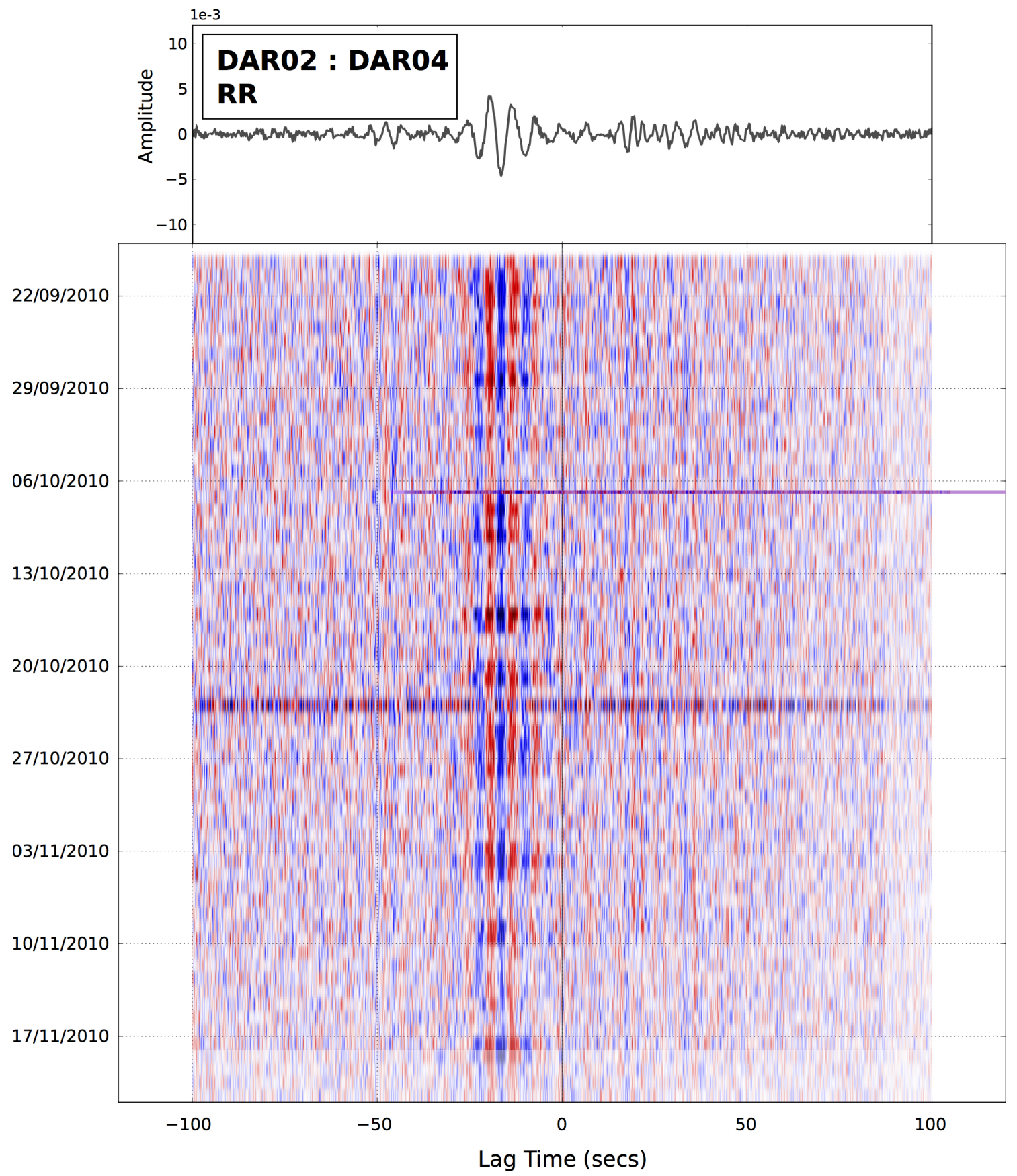

Figure 3.5: Daily RR correlation functions of station pair DAR02-DAR04 (see Fig. 3.1 for station locations), for a 2 month period from September 20th until November 20th 2010. The functions are normalised to the highest amplitude over the time period shown, with colour intensity proportional to amplitude. The top panel gives the corresponding stacked reference function for comparison. 
less dispersive moveout, with a dominant speed of approximately $2.5 \mathrm{~km} . \mathrm{s}^{-1}$. These components contain mostly Rayleigh waves. Love waves, visible on the TT record section (Fig. 3.6, right panel) have a highly dispersive moveout with speed ranging from $0.75 \mathrm{~km} . \mathrm{s}^{-1}$ to $3 \mathrm{~km} \cdot \mathrm{s}^{-1}$.

\subsubsection{Synthetic cross-correlation functions and higher mode surface waves}

First higher-mode Rayleigh waves have been observed in the Canterbury Plains region, on cross-correlations of the VUW-UWM-UA data (Savage et al. (2013); Figure 3.7). The presence of higher modes can cause computational difficulties and overestimated group and phase velocities in the automatic FTAN processing as the codes assume a single, fundamental mode surface wave (Levshin and Ritzwoller, 2001). It is therefore necessary to understand and define the surface wave phases exhibited in the functions so that suitable steps can be taken to account for and remove higher mode contamination. Figure 3.8 shows record sections of cross-correlations produced in this study filtered to the same $0.4-1 \mathrm{~Hz}$ bandwidth as Savage et al. (2013) and for the same range of interstation distances. This study includes several additional stations, and cross-correlations were produced across different instrument types, greatly increasing the total yield of correlation functions. Nonetheless, the record sections allow for a visual inspection of the consistency of cross-correlations between studies. For the vertical component correlation functions (Figure 3.8, top panels), a single Rayleigh wave mode with moveout slightly under $1 \mathrm{~km} . \mathrm{s}^{-1}$ is seen, assumed to be the fundamental mode. In contrast, the RR record section (Figure 3.8 , centre panels) additionally contain a faster arrival at $2 \mathrm{~km} . \mathrm{s}^{-1}$. These observations are consistent with those of Savage et al. (2013), who identified this faster Rayleigh wave as the first-higher mode.

For the dispersion analysis and tomography performed in this study, a different seismic-wave bandwidth is considered. Dispersion measurements are extracted for 2 $-20 \mathrm{~s}$, or $0.05-0.5 \mathrm{~Hz}$, lower than the frequencies studied previously with these data. Figure 3.9 gives the vertical and horizontal record sections for the lower frequencies. The surface-wave moveouts seen are similar to the broadband correlations shown previously (Figure 3.6). In contrast to the higher frequency record section, the radial-radial component shows a single moveout with a speed of approximately 2.5 $\mathrm{km} . \mathrm{s}^{-1}$ (Figure 3.9, centre panels). A likely explanation is that the longer periods (above $2 \mathrm{~s}$ ) are not as sensitive to the shallow low-velocity layer, which contains the surface-wave resonance for the shorter periods below $2 \mathrm{~s}$. The vertical component record section has moveout with the same speed (Figure 3.9, top panels), however 


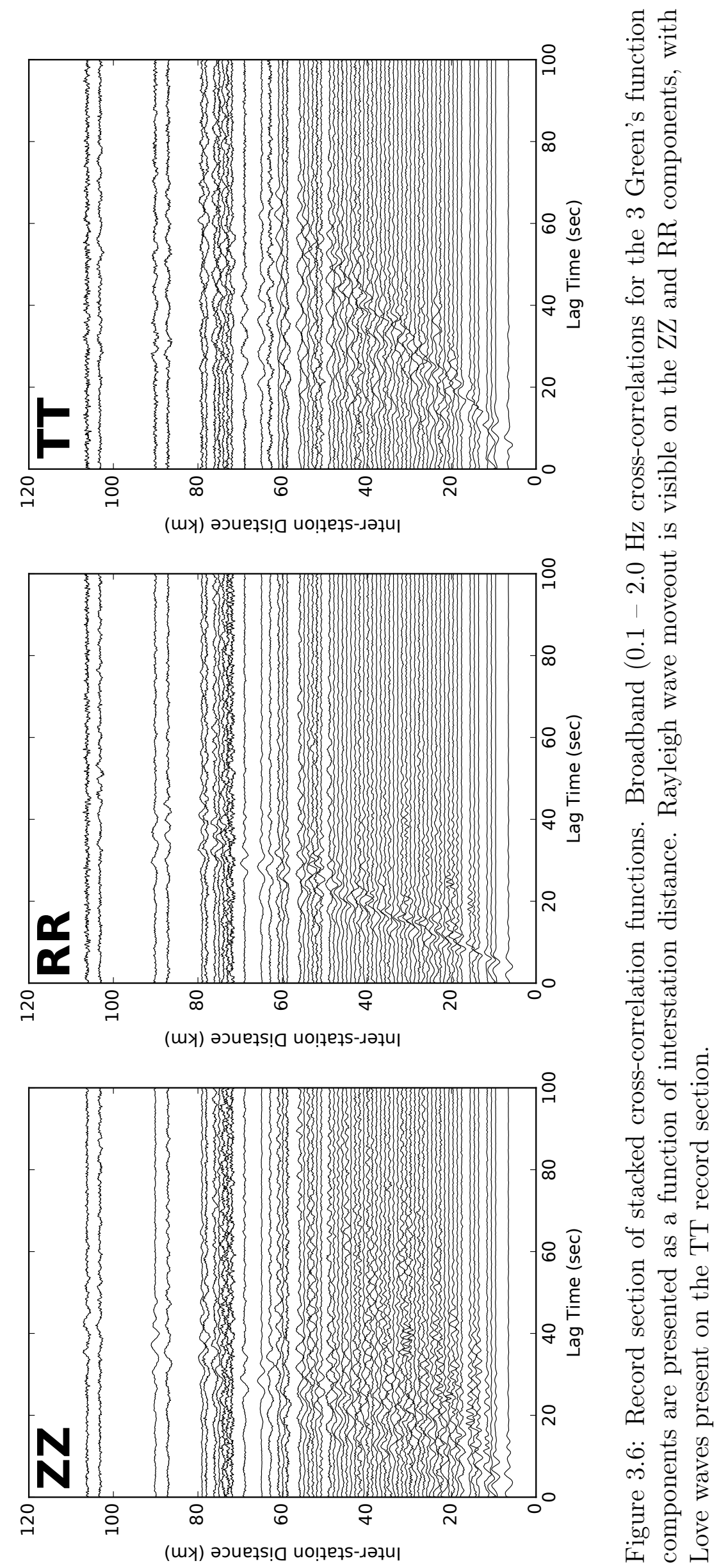



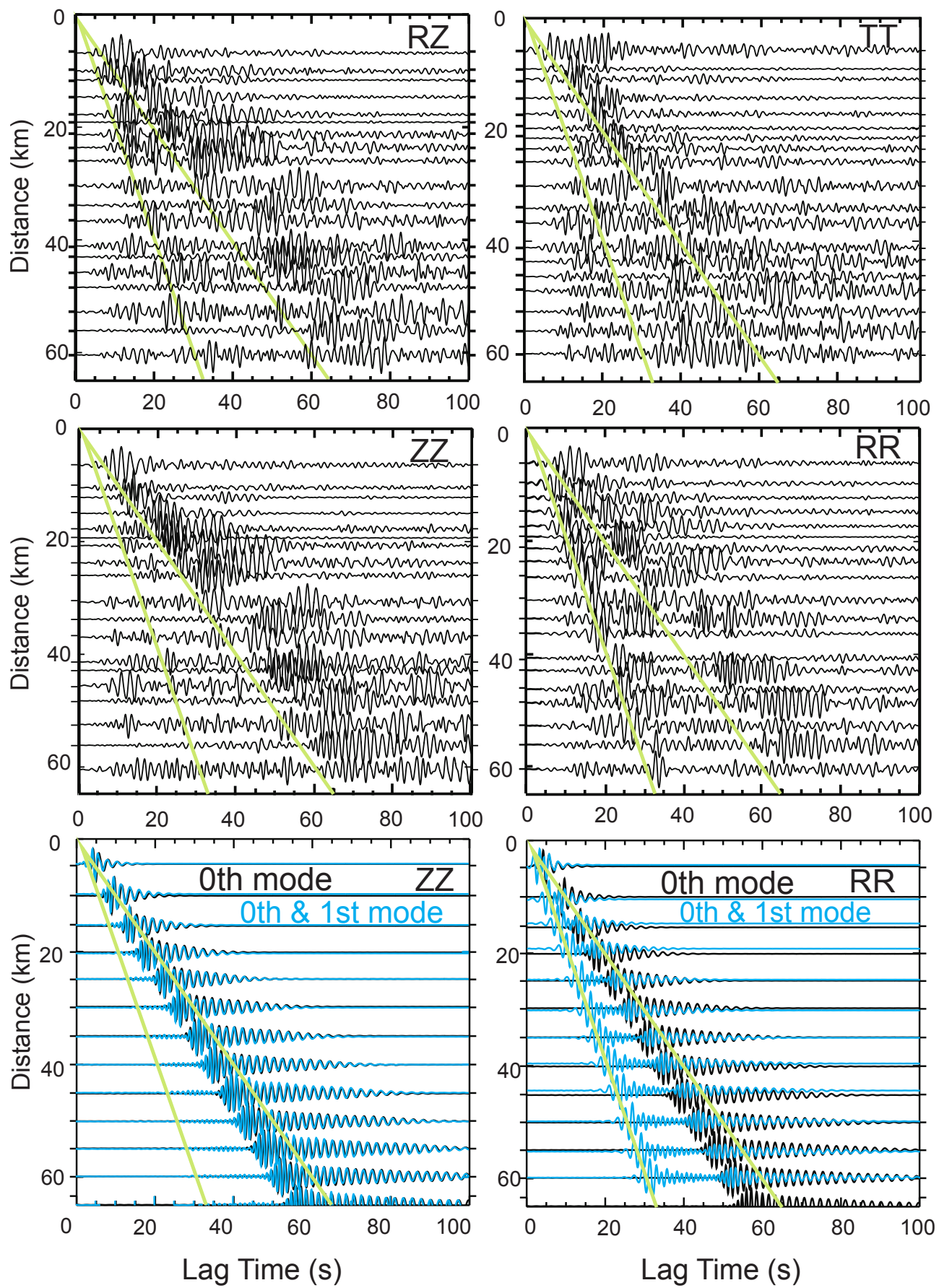

Figure 3.7: Record sections of Canterbury from Savage et al. (2013). High frequency correlations, filtered between $0.4-1.0 \mathrm{~Hz}$ are shown for several components (top and centre panels) with 1 and $2 \mathrm{~km} \cdot \mathrm{s}^{-1}$ moveout speeds annotated (yellow lines). The lower panels show ZZ and RR synthetic cross-correlations for a simplified shallow crustal velocity model to highlight fundamental and higher modes (black and blue functions respectively). 

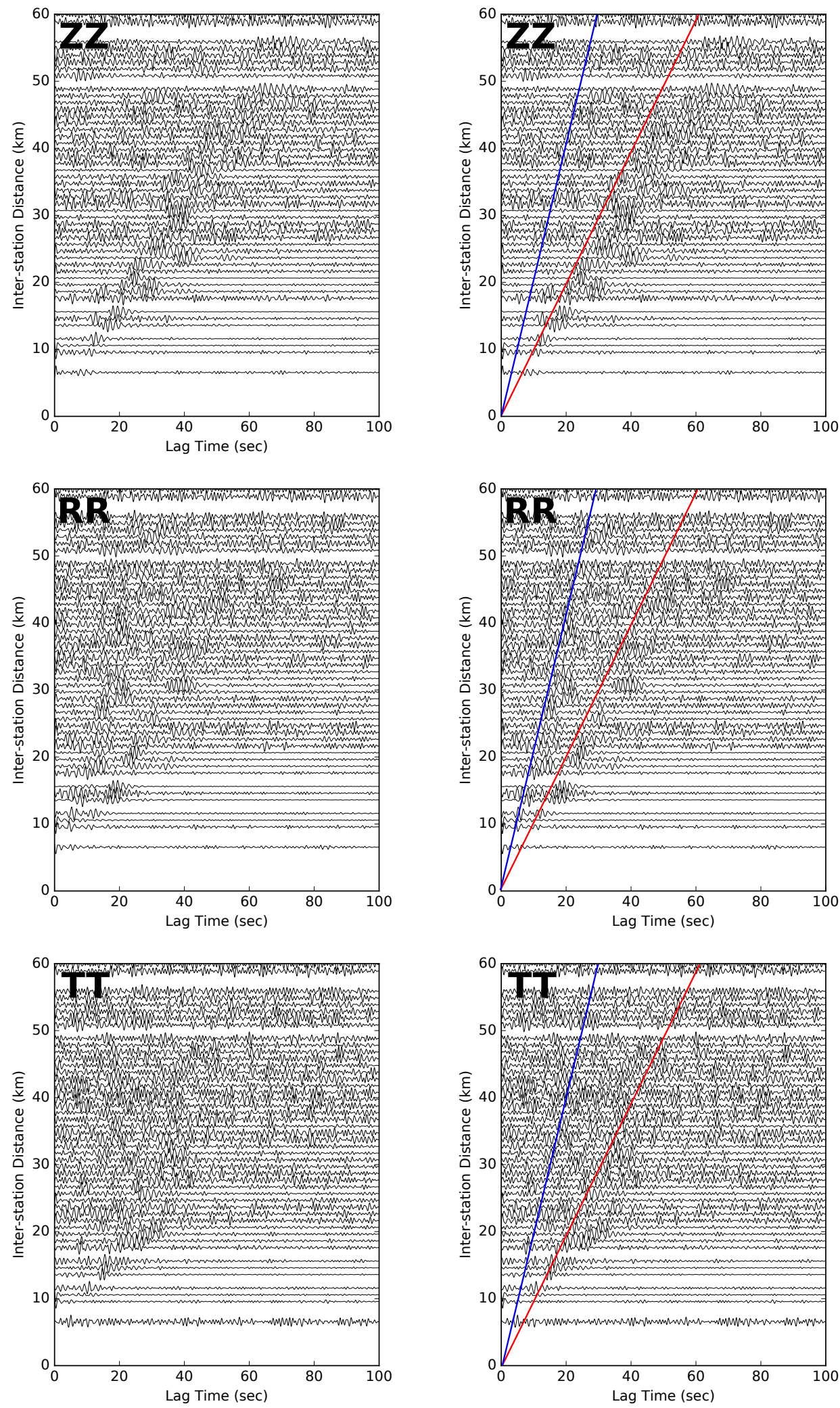

Figure 3.8: Record section of high frequency cross-correlation functions, filtered between $0.4-1 \mathrm{~Hz}$. Cross-correlations of the ZZ, RR and TT components as a function of interstation distance (left panels). The right panels illustrate the same record sections, with moveout speeds of $1 \mathrm{~km} \cdot \mathrm{s}^{-1}$ (red line) and $2 \mathrm{~km} . \mathrm{s}^{-1}$ (blue line) highlighted. 
it exhibits a more dispersive wave packet.

To be confident in our classification of the surface wave modes visible within this dispersion 2 - 20 s bandwidth, synthetic Green's functions have been produced, using the Computer Codes in Seismology package (Herrmann and Ammon, 2004). An average velocity model was used (Eberhart-Phillips et al., 2010, Appendix B.1) and a point force source located at a depth of $50 \mathrm{~cm}$. ZZ and RR component Green's functions were computed for fundamental mode Rayleigh waves only and with the first higher mode present. Each source-receiver distance considered corresponds to an inter-station distance present in the real cross-correlations. Vertical component functions assume a vertically propagating force recorded on the vertical seismometer component, and horizontal functions assume radial or transverse components recording radially propagating forces. Figures 3.10 and 3.11 show comparision between the synthetics produced using the velocity model of Savage et al. (2013) and this study, for the two considered frequency bands. The difference between the two sets of synthetics is the input models. In contrast to the multi-layered average model used in this study, Savage et al. (2013) assumed a $1.5 \mathrm{~km}$ low-velocity layer over a half-space, to approximate the sedimentary sequences overlying basement rocks in the Canterbury basin (Tables 3.2 and 3.3).

Table 3.2: Multi-layer velocity model for synthetic Green's function. This model was produced by averaging velocity models of Eberhart-Phillips et al. (2010) within the study region.

\begin{tabular}{cccc}
\hline Depth $(\mathrm{km})$ & $\mathrm{V}_{P}\left(\mathrm{~km} . \mathrm{s}^{-1}\right)$ & $\mathrm{V}_{S}\left(\mathrm{~km} . \mathrm{s}^{-1}\right)$ & Density $\left(\mathrm{kg} . \mathrm{cm}^{3}\right)$ \\
\hline 1.0 & 3.850 & 2.292 & 2.6 \\
3.0 & 5.012 & 2.923 & 2.6 \\
8.0 & 5.884 & 3.445 & 2.7 \\
15.0 & 6.139 & 3.602 & 2.8 \\
23.0 & 6.696 & 3.904 & 3.0 \\
48.0 & 8.324 & 4.836 & 3.4 \\
65.0 & 8.396 & 4.854 & 3.4 \\
85.0 & 8.405 & 4.859 & 3.4 \\
105.0 & 8.436 & 4.875 & 3.4 \\
130.0 & 8.463 & 4.892 & 3.5 \\
155.0 & 8.420 & 4.865 & 3.4 \\
185.0 & 8.473 & 4.897 & 3.4 \\
225.0 & 8.560 & 4.950 & 3.5 \\
\hline
\end{tabular}

Figure 3.10 shows synthetics for the higher frequency band of $0.4-1 \mathrm{~Hz}$. The top panels are produced by the model of Savage et al. (2013) and lower panels are produced with the model of Eberhart-Phillips et al. (2010). With a shallow lowvelocity layer present, the first higher mode is easily distinguished on the RR Green's 

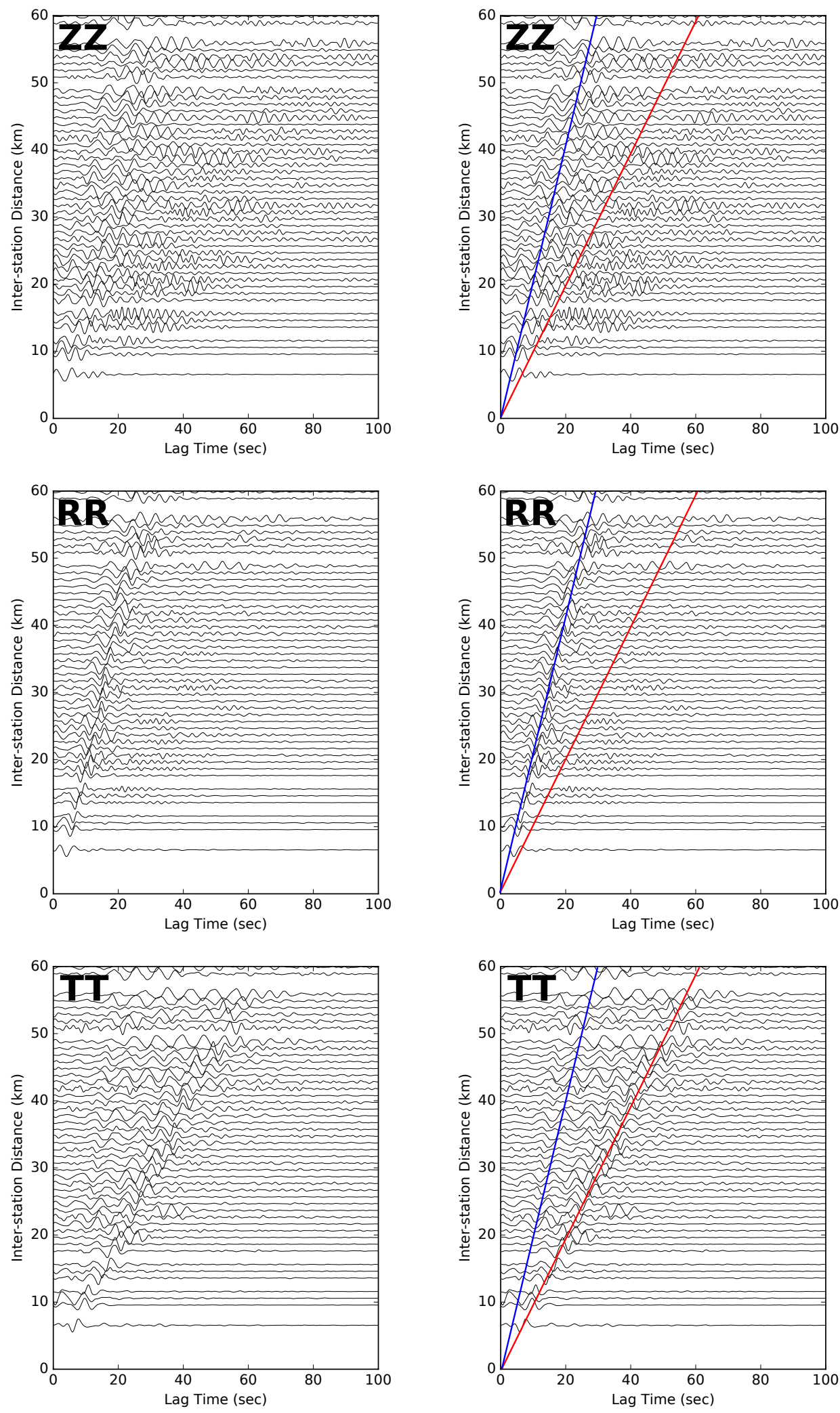

Figure 3.9: Low frequency record sections of ZZ, RR and TT components, filtered to $0.05-0.5 \mathrm{~Hz}$ (or $2-20 \mathrm{~s}$ ). Cross-correlations of the ZZ, RR and TT components as a function of interstation distance (left panels). The right panels illustrate the same record sections, with moveout speeds of $1 \mathrm{~km} . \mathrm{s}^{-1}$ (red line) and $2 \mathrm{~km} . \mathrm{s}^{-1}$ (blue line) highlighted. Cross-correlations within this frequency band are taken forward and considered for dispersion analysis. 
Table 3.3: Simple two-layer model for synthetic Green's functions computation, after Savage et al. (2013).

\begin{tabular}{cc}
\hline $\operatorname{Depth}(\mathrm{km})$ & $\mathrm{V}_{P}\left(\mathrm{~km} . \mathrm{s}^{-1}\right)$ \\
\hline 0 & 2.400 \\
1.5 & 5.400 \\
\hline
\end{tabular}

functions. When this layer is not included in the synthetic models, only a single moveout is present (Figure 3.10, left panels). Comparing this to Figure 3.8, the RR cross-correlations do have two moveouts present, confirming the presence of a thin low-velocity layer and the first higher mode.

Considering the low frequency band used for the dispersion analysis in this study, synthetic Green's functions were also produced with both input models (Figure 3.11). The top panels are produced by the model of Savage et al. (2013) and lower panels are produced with the model of Eberhart-Phillips et al. (2010). With a low-velocity layer present, the RR Green's functions have a fast moveout of approximately 2.5 $-3.0 \mathrm{~km} . \mathrm{s}^{-1}$ for the fundamental mode, compared to a slower and more diffuse $\mathrm{ZZ}$ surface wave arrival (Figure 3.11, top panels). The presence of the first higher mode does not result in a faster moveout speed. With the models of Eberhart-Phillips et al. (2010), a single dominant moveout of approximately $2.5-3.0 \mathrm{~km} . \mathrm{s}^{-1}$ is seen on both components, even when the first higher mode was included in the synthetic Green's function generation. The lack of a clear higher mode, as opposed to the synthetics of Savage et al. (2013), is due to a more complex velocity model at depth, with less shallow subsurface resolution. The velocity contrast with the uppermost layers was also less than that of Savage et al. (2013). If the synthetics are again compared to the real cross-correlations for the same frequency band (Figure 3.9), we can see that the $\mathrm{ZZ}$ component has a diffuse moveout, whereas the radial-radial component has a sharp, fast moveout of approximately $2.0 \mathrm{~km} \cdot \mathrm{s}^{-1}$.

It is therefore likely that the dominant surface wave packet in the radial-radial component is the fundamental mode in the frequency band of interest. The real cross-correlations also most resemble the synthetics when a low-velocity surface layer is present, which will be an important consideration for later shear-wave inversion processing. 

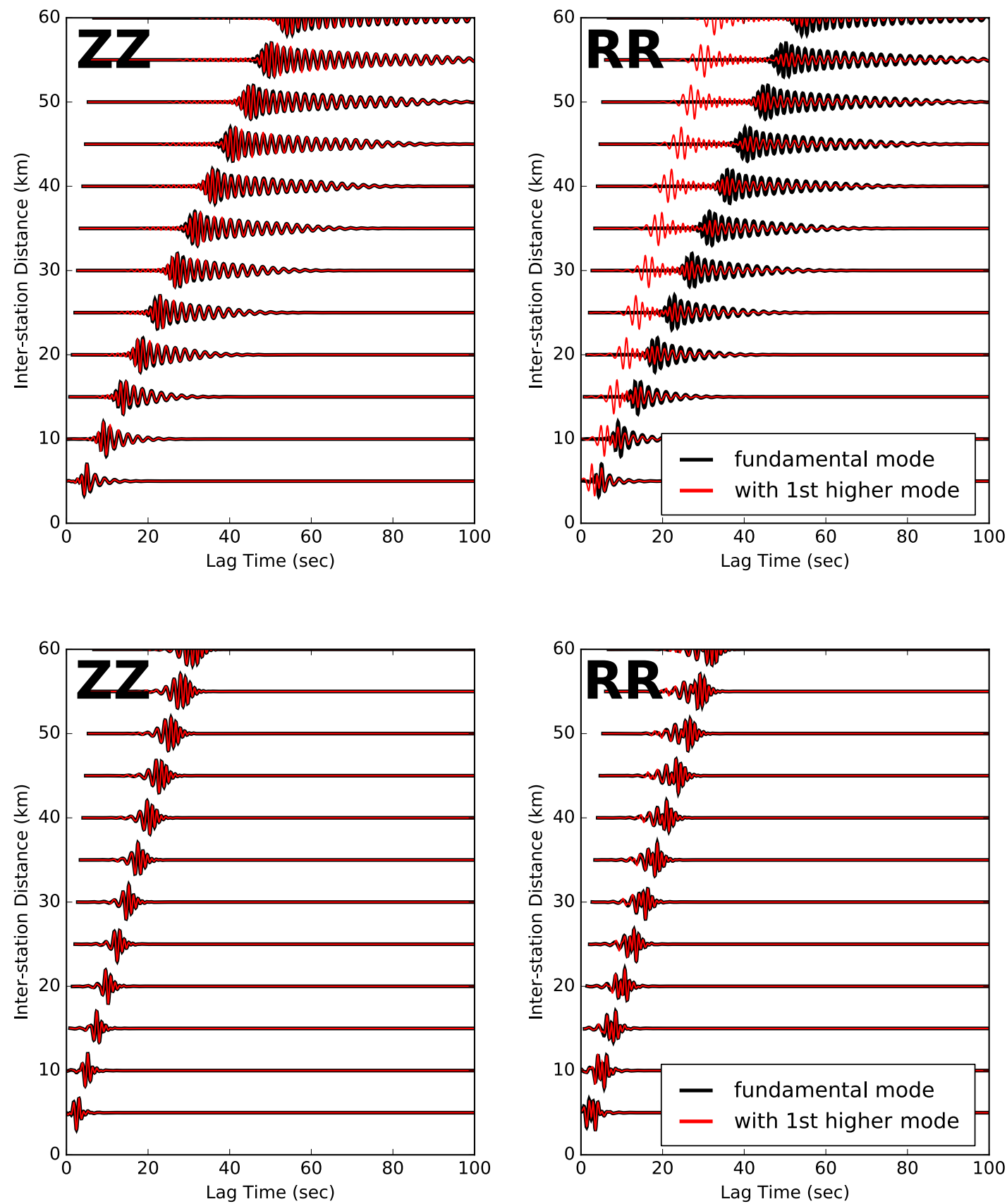

Figure 3.10: High frequency synthetic Green's functions for the vertical and radial components, filtered between $0.4-1 \mathrm{~Hz}$, to look for potential evidence of higherorder Rayleigh wave modes. Top panels are generated from the model of (Savage et al., 2013) and lower panels are based on a multi-layer average regional velocity model (Appendix B), synthetic Green's functions are shown to contain the fundamental mode Rayleigh wave only (black) and with the first-order higher mode present (red). 

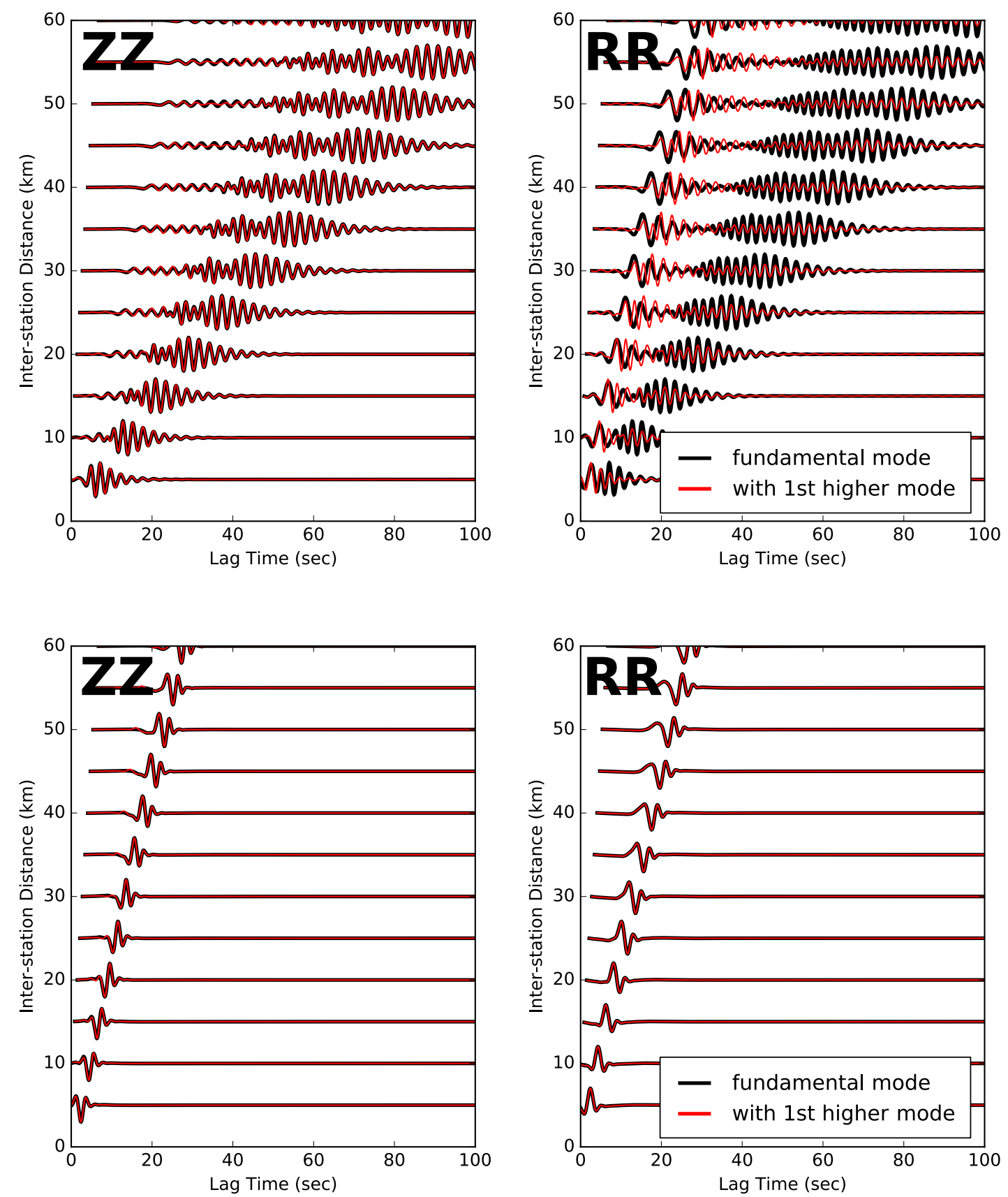

Figure 3.11: Low frequency synthetic Green's functions for the vertical and radial components, filtered between $0.05-0.5 \mathrm{~Hz}$, to look for potential evidence of higherorder Rayleigh wave modes. Top panels are generated from the model of (Savage et al., 2013) and lower panels are based on a multi-layer average regional velocity model (Appendix B), synthetic Green's functions are shown to contain the fundamental mode Rayleigh wave only (black) and with the first-order higher mode present (red). 


\subsubsection{Canterbury dispersion}

\section{Signal-to-noise ratios across the Canterbury Plains}

The signal-to-noise ratio (SNR) of each cross-correlation function is calculated as a measure of quality control prior to dispersion analyses. Two approaches were considered, firstly the SNR of the whole function was computed, and secondly the functions were filtered in several narrow bands and a SNR is calculated for each band (for method see Section 2.6). Considering the SNR of the whole function, between $0.1-2 \mathrm{~Hz}$, there is a wide distribution of values up to 26 (Figure 3.12). Any station pairs giving a SNR of less than 4 were discarded from dispersion analysis. This threshold was chosen after a visual inspection of low SNR correlations, to allow the maximum number of cross-correlations for dispersion analysis, whilst still ensuring high quality surface wave signals. Figure 3.13 indicates that the SNR is typically highest at periods of approx $4-8 \mathrm{~s}$. It was decided that below $4 \mathrm{~s}$ the SNR is too low to be confident of picked dispersion, so measurements are not computed in this range. The SNR for different cross-correlation components for individual pairs is examined. Some examples are presented in Figure 3.14. The transverse-transverse component commonly contains the lowest signal-to-noise ratios, and all components have low-amplitude signals below $4 \mathrm{~s}$.
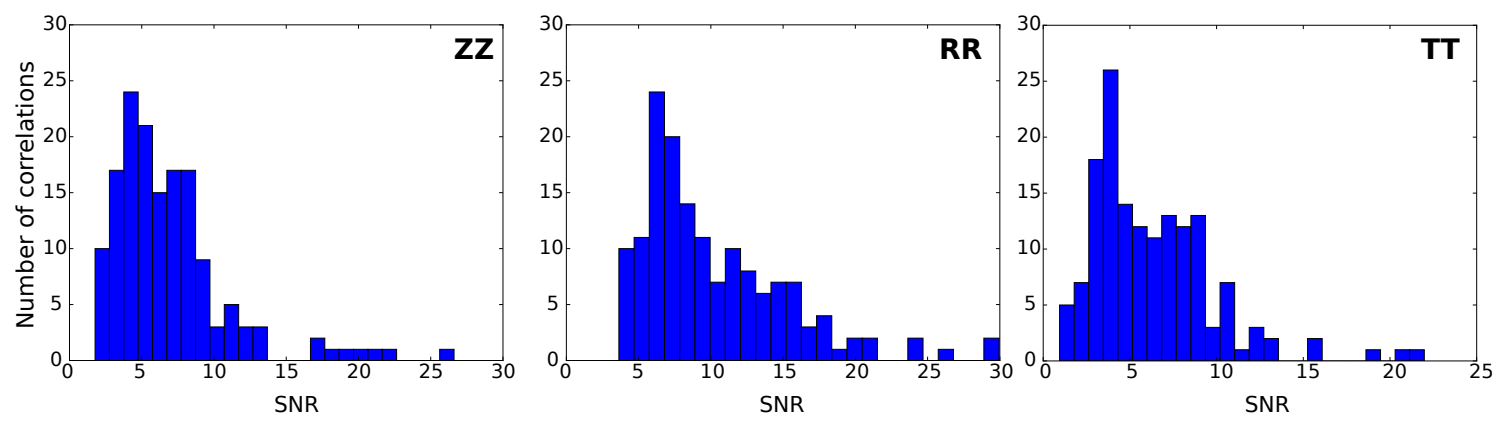

Figure 3.12: Histograms of SNR's versus the number of station pairs for the ZZ, RR and TT components, illustrating the distribution of signal-to-noise ratios.

All computed group and phase velocity dispersion curves are presented in Figure 3.15 for periods between 4-10 s. There is a wide distribution of group velocity curves (left panels). Following examination of all dispersion curves in conjunction with signal-to-noise ratios, several stations consistently gave unreliable and low quality results. Picked phase velocity curves show two distinct velocity trends; below and above $1.5 \mathrm{~km} . \mathrm{s}^{-1}$. It was decided to remove all stations pairs with stations CTND and MCHD of the GeoNet temporary deployment and the permanent RPZ station. CTND and MCHD were extremely noisy stations, which resulted in the low signal-tonoise ratios of any Green's functions from them. RPZ is situated outside the region 


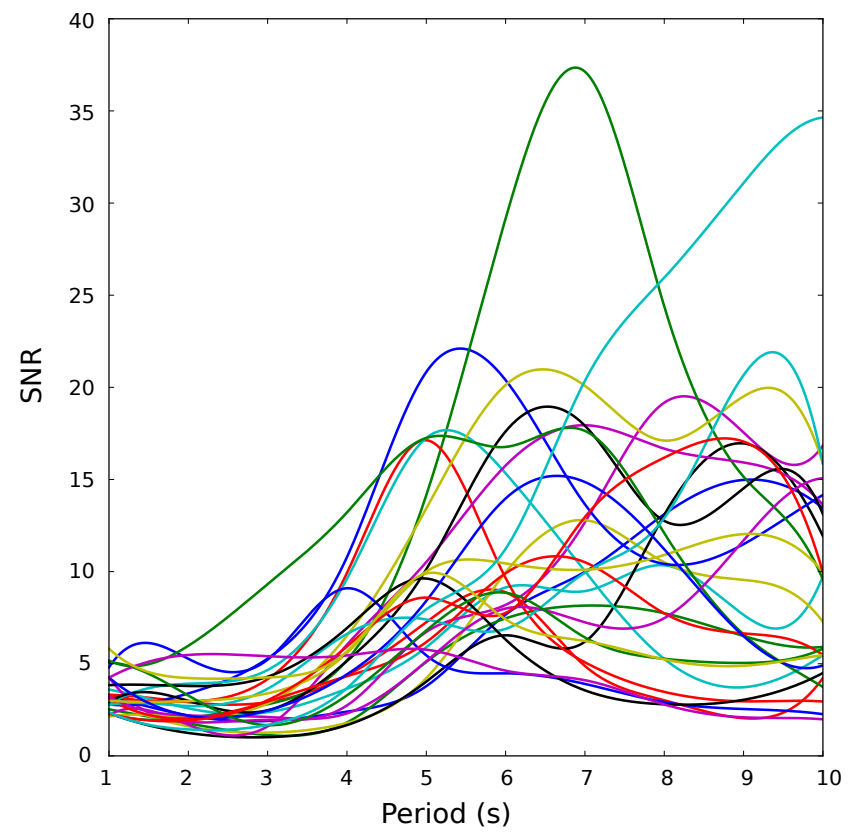

Figure 3.13: Signal-to-noise ratios as a function of period for vertical crosscorrelations of all station pairs of DAR01 to DAR08 for 1 - $10 \mathrm{~s}$. SNRs are consistently low for periods below $4 \mathrm{~s}$ and thus are not considered in dispersion analyses.
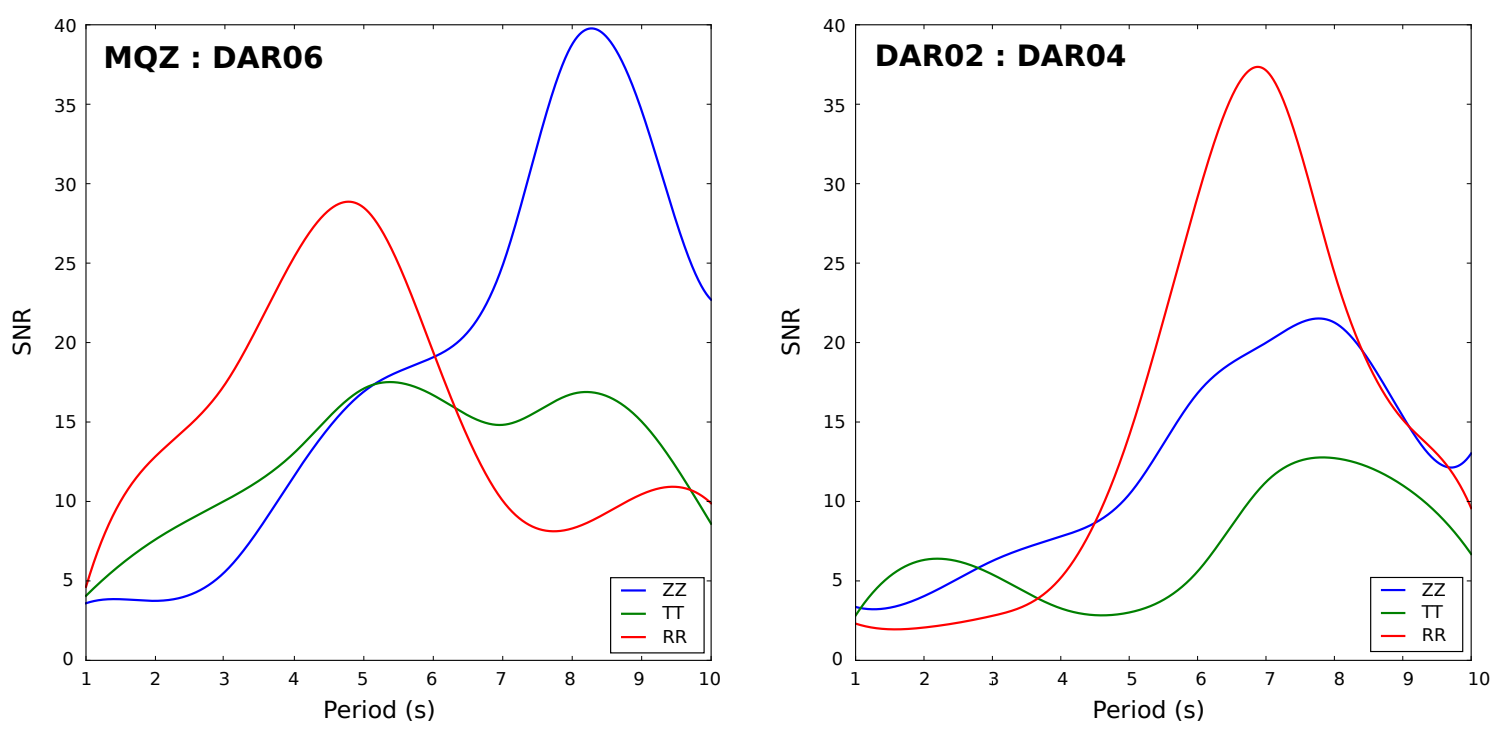

Figure 3.14: Comparison of SNR as a function of period, for the three crosscorrelation components. Two example station pairs are presented; MQZ-DAR06 (left panel) and DAR02-DAR04 (right panel) showing the ZZ (blue), RR (red) and TT (green) components. 
where all other stations are located, approximately $40 \mathrm{~km}$ from DAR01, which is the next westernmost station. Having a station located far away from all others creates velocity smearing along the station-paths in the tomography models, in the case of $\mathrm{RPZ}$, this would be in an E-W direction, in addition to low resolution from the small number of station pairs in the region to the west of DAR01. The most likely reason for the poor quality dispersion measurements from RPZ is the larger interstation distances coupled with the short duration of the stacked correlation functions. The further surface waves travel, the greater the effect of attenuation of the signal.

As discussed in Section 2.6, a wavelength constraint was also placed on the crosscorrelations. The dispersion curves for station pairs with an interstation distance less than two wavelengths apart were removed from subsequent processing.

After application of the quality control measures, the dispersion curves shown in Figure 3.16 were taken forward to the tomographic inversions. Considering the group velocity curves for all components (left panels), the range of measured velocities has not changed drastically from Figure 3.15, however curves with spurious points have been removed. The number of phase velocity curves has been greatly reduced (Figure 3.16 , right panels), however the spread of velocities has been reduced and all curves that previously showed velocities below $1.5 \mathrm{~km} . \mathrm{s}^{-1}$ have been removed.

A measure of the uncertainty in the dispersion curves can be approximated, by examining the picked dispersion curves for cross-correlations stacked over the whole study period, compared to stacks of shorter duration. Figure 3.17 shows such examples for two station pairs: DAR02-DAR07 and MQZ-OXZ. The vertical component dispersion curves for monthly stacks (black lines) are compared to that of the overall stacked cross-correlation (red lines). For both station pairs the spread of picked velocities is approximately $0.1 \mathrm{~km} . \mathrm{s}^{-1}$, for periods below $7 \mathrm{~s}$. Higher periods demonstrate larger variations.

\section{Spatial variation}

Prior to tomographic and shear wave inversions, the dispersion curves are examined in subsets to try to see spatial differences in seismic velocities across the region. Several subsets of ZZ group velocity curves are shown in Figure 3.18. Comparing correlations with station pairs of different networks, such as the VUW-UWM-UA (top left panel) and GeoNet permanent (bottom left) and GeoNet temporary (bottom right) there do not seem to be any systematic differences present. There is a wide spread for cross-correlations across different networks (e.g. Figure 3.18, bottom left) suggesting that instrument responses were correctly removed. Station paths that run along the Greendale Fault tend to have lower than average group 

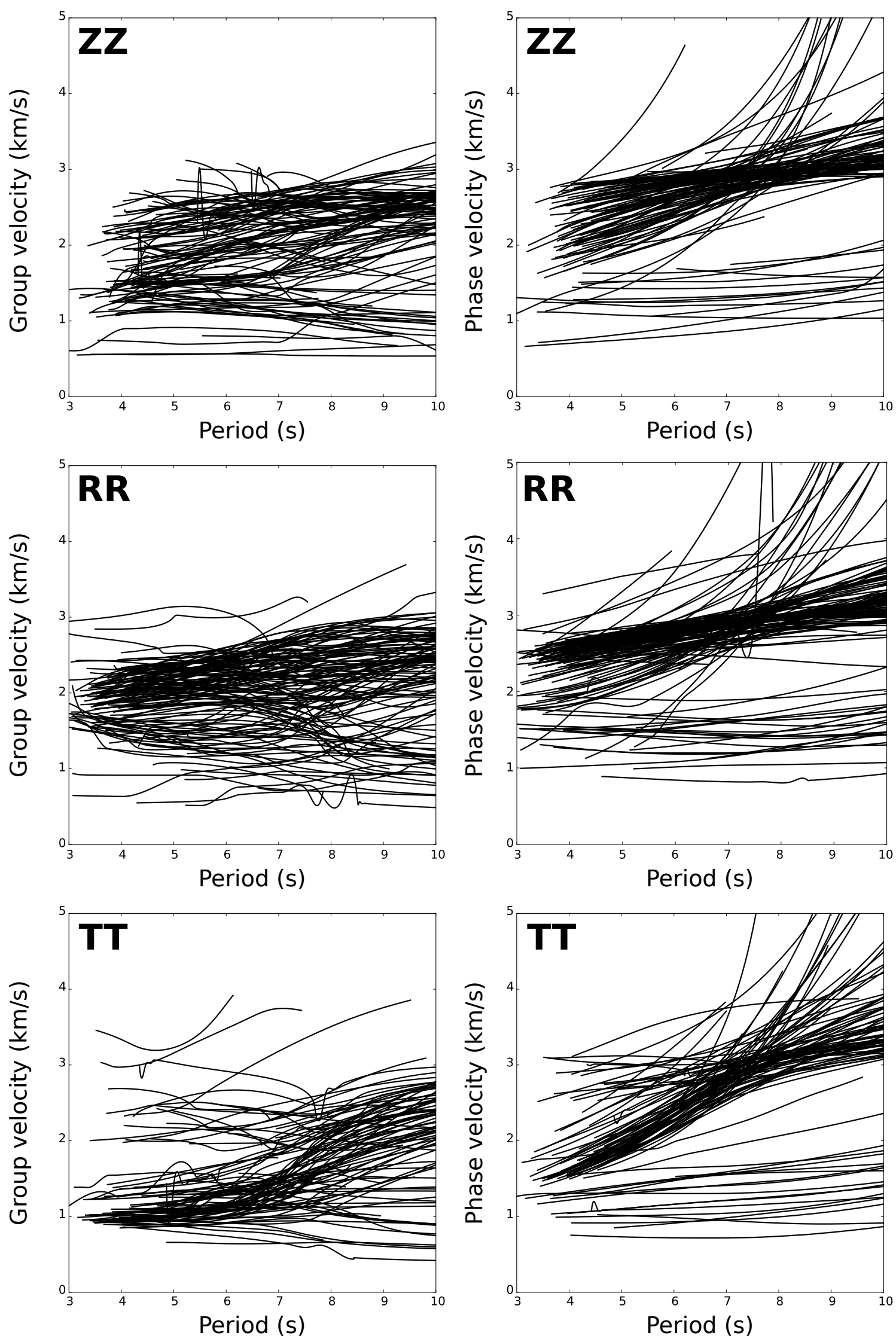

Figure 3.15: Dispersion curves computed from AFTAN processing. Group (left panels) and phase (right panels) velocities as a function of period are plotted for all station pairs, for cross-correlation components ZZ, RR and TT. All calculated dispersion curves are shown here, prior to applying quality control critera. 

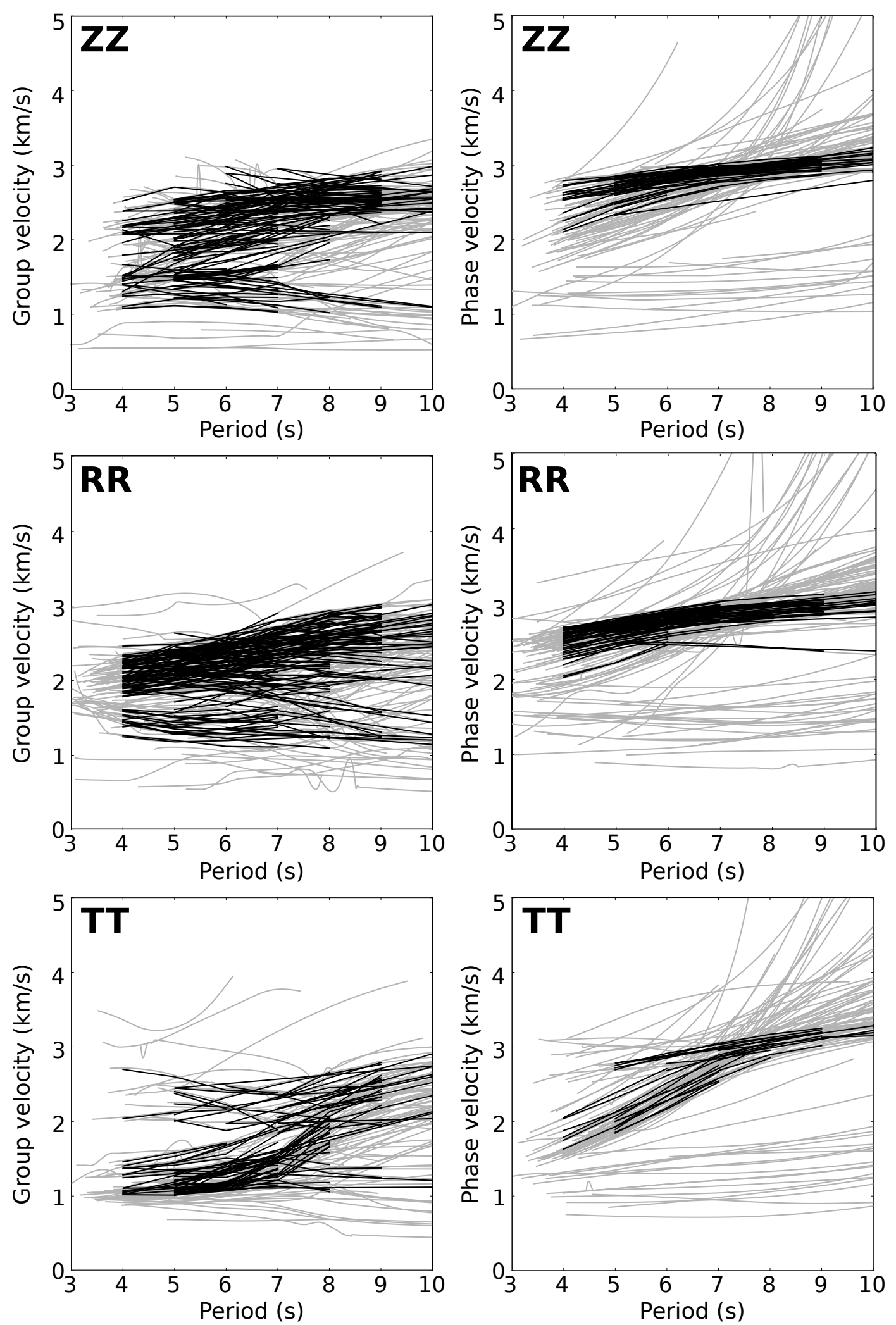

Figure 3.16: Group (left) and phase (right) velocity dispersion curves to be used in tomographic inversions are shown in black, following several quality control measures. A minimum SNR of 4 was required for all station pairs in addition to cut-off periods greater than 2 wavelengths, up to a maximum period of $10 \mathrm{~s}$. Light grey lines show the rejected dispersion curves. 

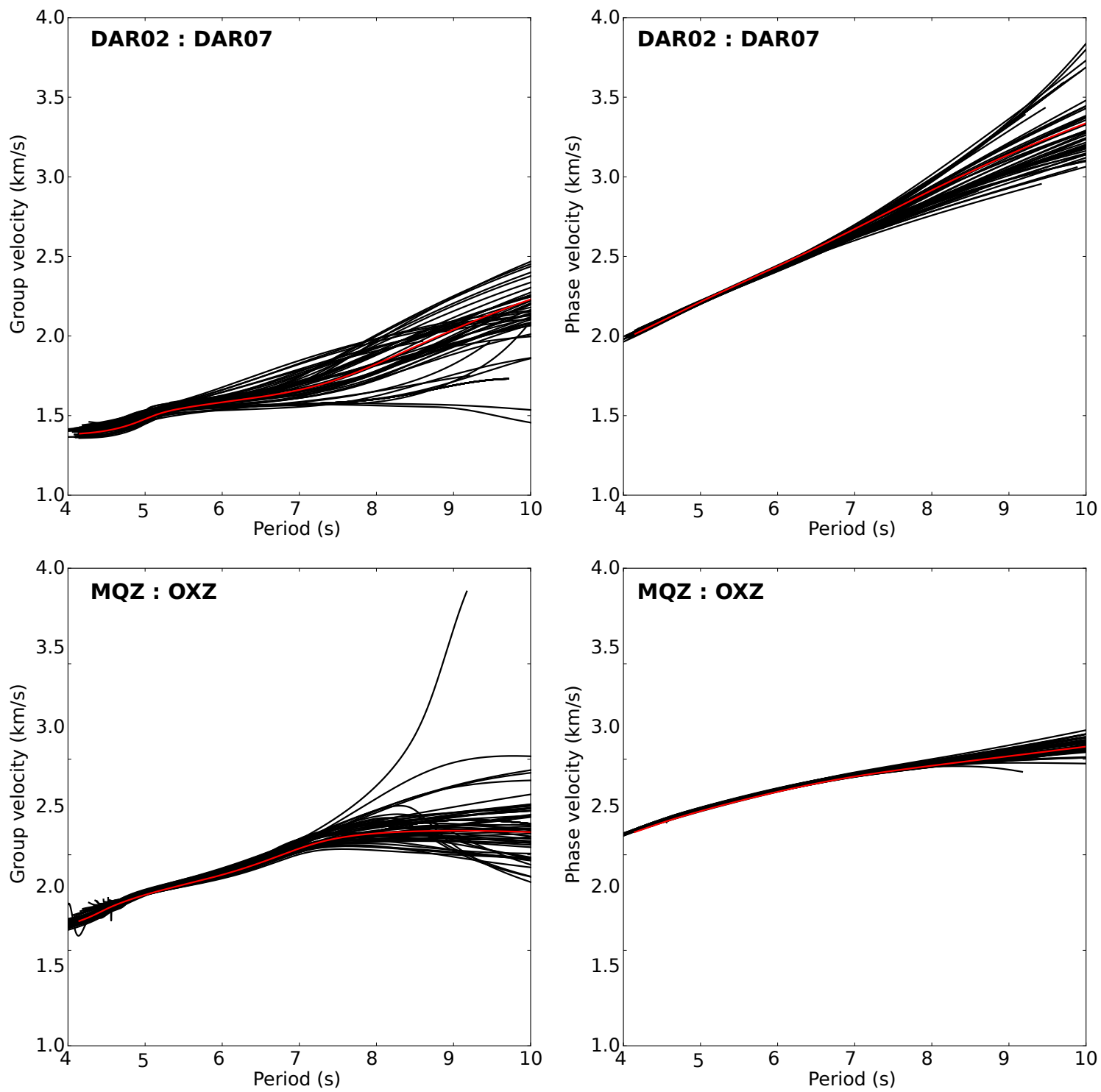

Figure 3.17: Dispersion curves for month-long stacks for station pairs DAR02DAR07 and MQZ-OXZ. Group (left) and phase (right) velocity measurements are shown for each monthly vertical component cross-correlation function. The final picked dispersion curve from the stack spanning the whole study period is plotted in red. 
velocities, and although only 4 station pairs passed the quality control measures, the observation could be suggestive of a low-velocity region along the fault.

\subsubsection{Surface wave tomography}

Following application of all quality control measures, the accepted dispersion curves are taken forward for tomographic inversions. Due to the wavelength constraint, which requires a minimum interstation distance of 2 wavelengths, at longer periods, less dispersion measurements are accepted as the number of paths with distances greater than 2 wavelengths decreases. This results in decreased path coverage, and therefore lower spatial resolution in the tomography models is to be expected with increasing period. Figure 3.19 shows all station paths highlighted where a measurement for Rayleigh wave phase velocity for each period is available, with a markedly decreased path coverage at longer periods. In addition to the quality controls described above, bounds are placed on expected surface wave velocities, to further remove any spurious dispersion measurements. Velocities less than $1.0 \mathrm{~km} . \mathrm{s}^{-1}$ and greater than $5.0 \mathrm{~km} . \mathrm{s}^{-1}$ are removed.

As described in Section 2.4.2 and in greater detail by Barmin et al. (2001), two penalty functions are minimised during the inversion to achieve the lowest model misfits and highest spatial resolution by down-weighting nodes with low path coverage The damping parameters; $\alpha$ and $\sigma$ are chosen based on systematic testing of various combinations of the two, and examination of the output models and associated misfits. Smearing, spatial resolution and the ability of each model to resolve expected velocity features are considered. The values chosen to be most appropriate are $\alpha=200$ and $\sigma=25$. The inversion uses a $0.1^{\circ} \times 0.1^{\circ}$ grid throughout the study region.

A resolution matrix for the models is calculated with each inversion, using the method of Barmin et al. (2001). At each nodal point a cone, which is the response of the inversion to a $\delta$-shaped perturbation, is fitted to the resolution matrix. The spatial resolution is given by the radius of the cone. In simpler terms, the resolution gives the distance at which two $\delta$ perturbations can be fully resolved Barmin et al. (2001). In contrast to the commonly used checkerboard resolution test, the spatial resolution at each nodal point in the tomographic inversion is quantified. Figure 3.20 shows the resolution analysis for periods $5-10 \mathrm{~s}$ for Rayleigh phase velocities. The spatial resolution in kilometres is shown to a maximum of $40 \mathrm{~km}$, and a minimum of twice the distance between nodes, which in this case is $2 \times 0.1^{\circ} \approx 22 \mathrm{~km}$. Where resolutions in kilometres are low, there is high spatial resolution in the tomographic inversions. For all periods there is high spatial resolution within the extent of seismic 

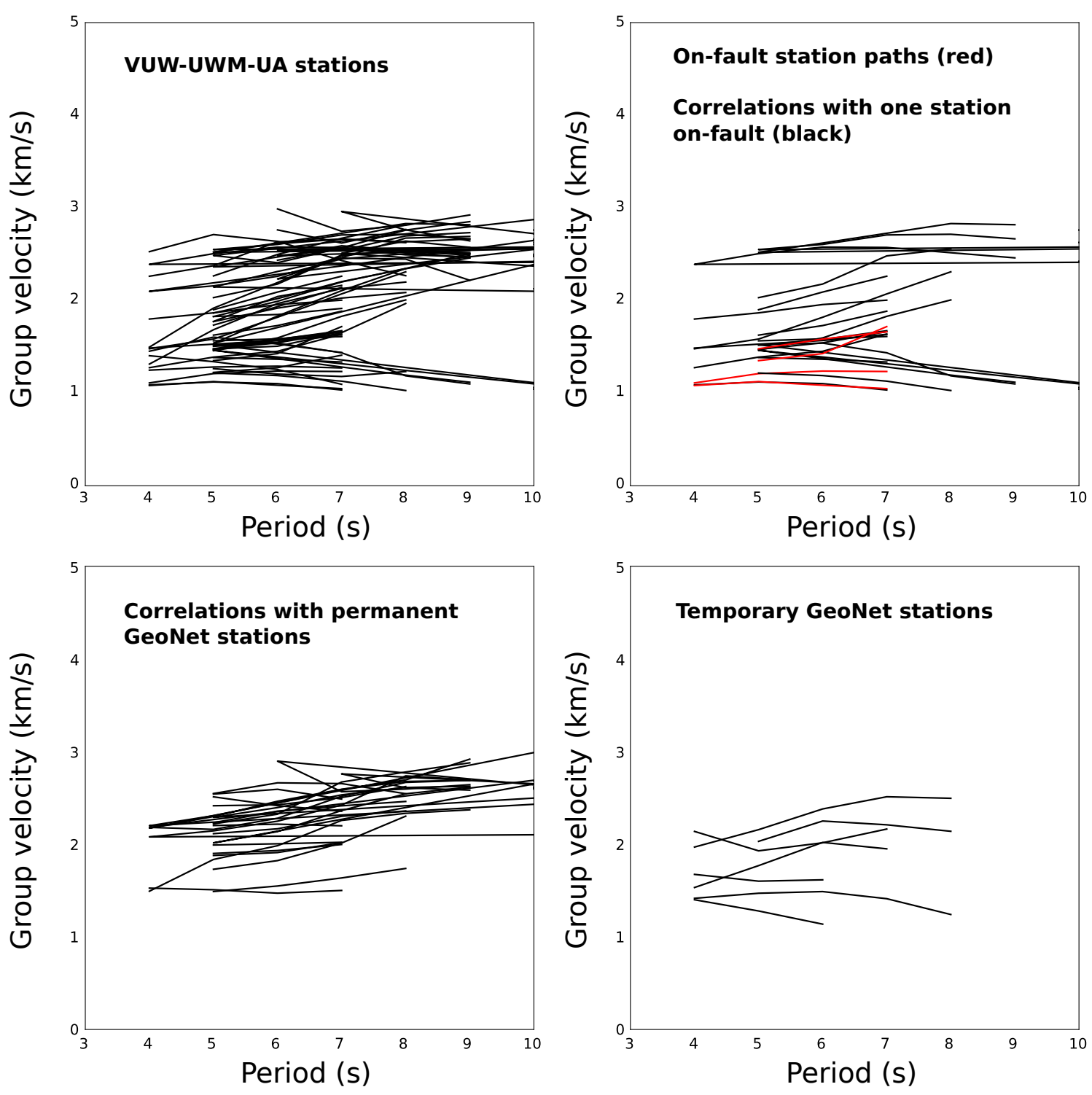

Figure 3.18: Vertical component group velocity measurements for several station pair subsets. Dispersion curves are shown grouped into several subsets to examine systematic variations throughout the region. In the top right panel, dispersion curves from correlations of on-fault station pairs are highlighted in red, alongside curves with one station situated on the Greendale Fault. 

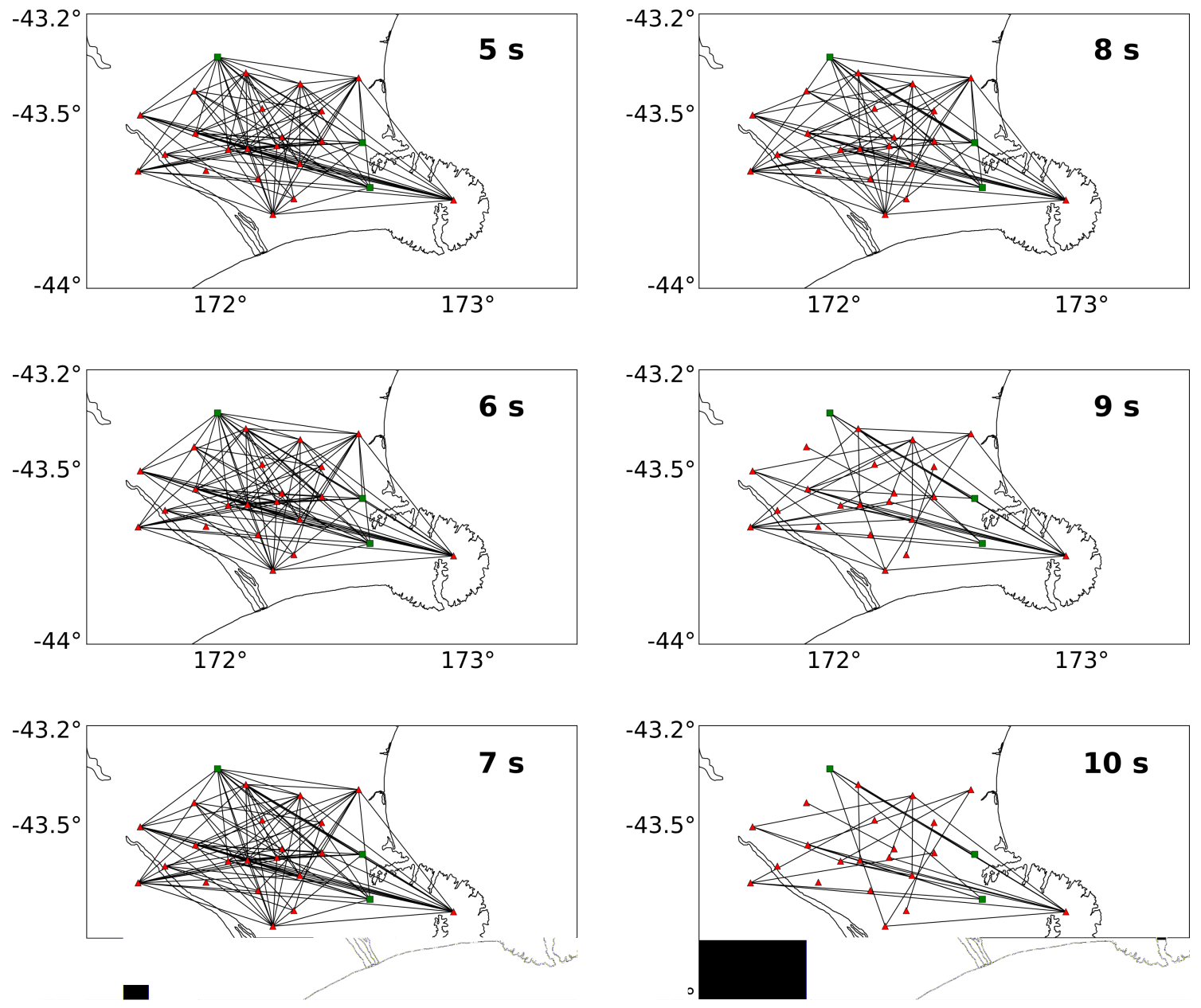

Figure 3.19: Interstation path coverage for the Canterbury region, as a function of period. Each available interstation path is plotted for periods $5-10 \mathrm{~s}$. It is seen that for increasing periods, fewer measurements are allowed to tomographic inversions, due to measurements not meeting quality control measures. 

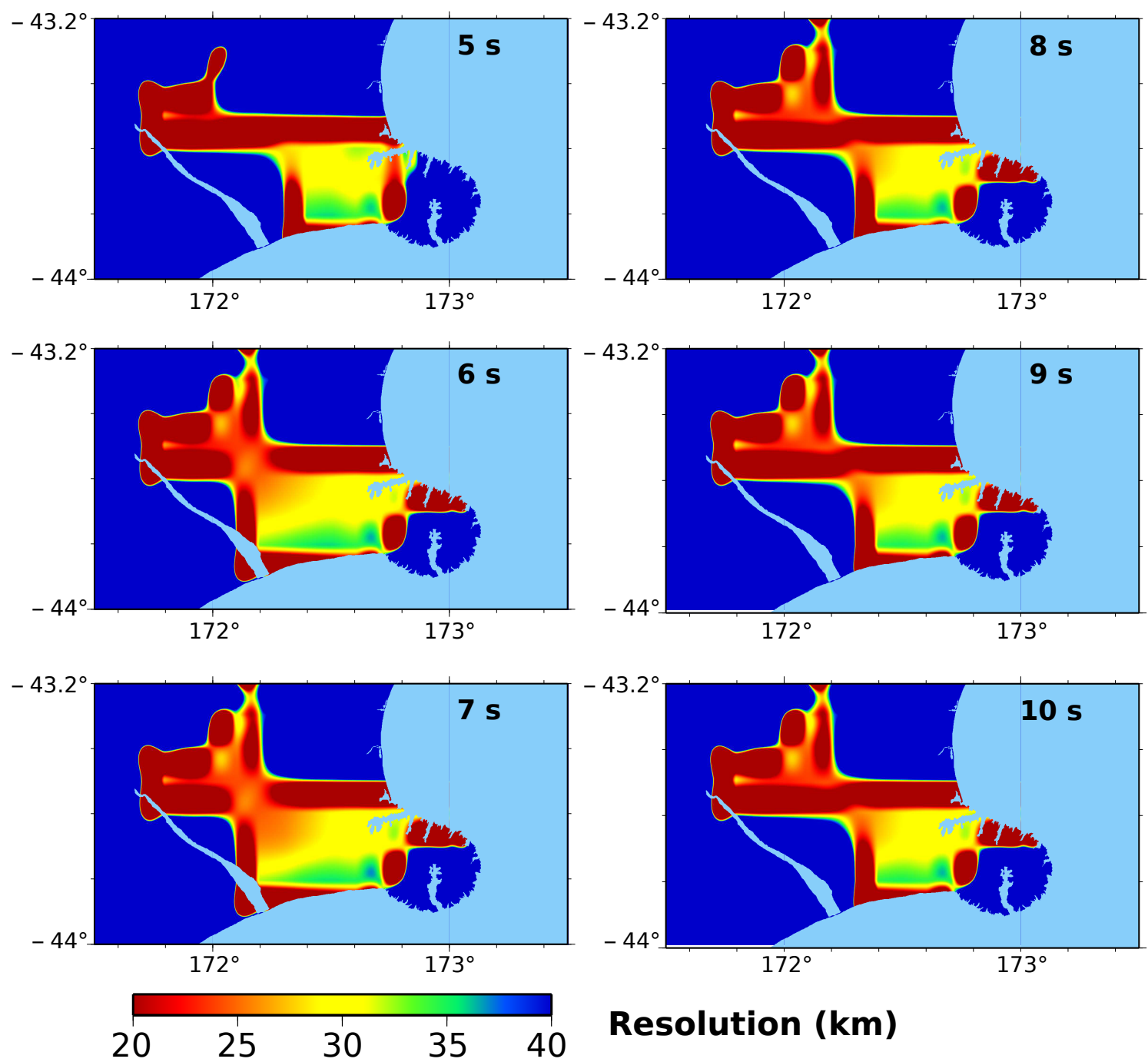

\section{Resolution (km)}

Figure 3.20: Spatial resolution analysis of Canterbury tomography maps. For periods of $5-10 \mathrm{~s}$, the spatial resolution is calculated as described in Section 3.3.4 and mapped, to a maximum spatial resolution of $40 \mathrm{~km}$. The resolution in kilometres describes the minimum distance at which a $\delta$-perturbation can be fully resolved. Resolutions at points greater than $40 \mathrm{~km}$ have been set at this maximum in the figure, to easily visualise the regions of good resolution.

stations. There is some smearing in an E-W direction through the centre of the region, following the approximate location and strike of the Greendale Fault. This smearing is due to the high density of stations located on the fault, resulting in a large number of interstation paths along strike.

Another method to quantify the resolution of the tomographic inversions is to examine the azimuthal coverage throughout the region. The range of azimuths from 0 to $180^{\circ}$ of paths travelling through each model cell is calculated and presented in Figure 3.21. The highest azimuthal sampling of up to $80^{\circ}$ is seen to the north of the region. Lower azimuthal coverage in the south of the area, below approximately $-43.6^{\circ}$ latitude, is due to fewer stations to the south of the Greendale Fault, and 

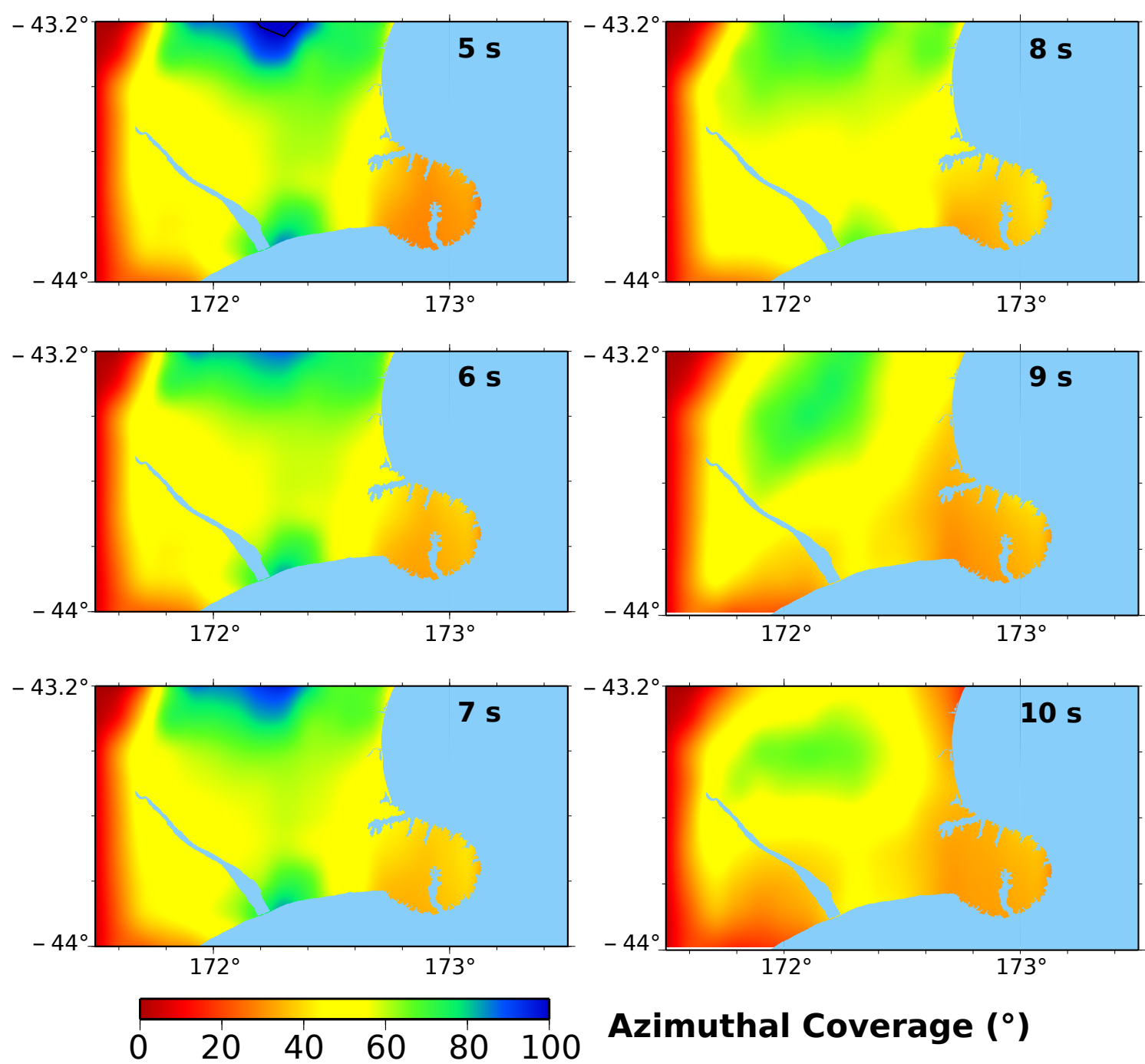

\section{Azimuthal Coverage $\left({ }^{\circ}\right)$}

Figure 3.21: The azimuthal path coverage at each nodal point in the tomographic inversions is calculated for periods $5-10 \mathrm{~s}$.

most station paths here striking E-W (see also figure 3.19).

\subsubsection{Shear wave inversion}

Inversions for shear velocity can be approached in several ways. Dispersion curves measured directly from the output of AFTAN analyses (Section 3.3.3) can be inverted, however the resulting models are averages for the interstation path, and so are imprecise where the interstation path is large (see Section 2.4.3). A second approach is to obtain dispersion curves at each nodal point by interpolating between tomography models. This results in dispersion curves every $0.1^{\circ}$ which are inverted for 1-D shear velocity models. Inversions for some station pairs that lie close to each other have been produced and these path-averaged models are compared to those of nearby grid points as a quality control measure. 
The inversion procedure relies heavily on the parameterisation of the starting model. It is desirable to set parameters with bounds that are wide enough to search the whole parameter space and not get trapped within local minima, but narrow enough to allow the models to converge to the minimum misfit and avoid unstable inversions. It is also important to avoid over-parameterising the model and placing too many constraints on the inversions. A priori knowledge of average expected shear velocities from previous models (e.g. Eberhart-Phillips et al., 2010; Syracuse et al., 2013), in addition, the synthetic seismograms were used to help decide on appropriate parameters. Two layers over a halfspace are modelled to a depth of $10 \mathrm{~km}$, with a shallow, low-velocity layer representing the sedimentary cover throughout the Canterbury Plains. Table 3.4 summarises the input for inversions within the region. Wide ranges for $\mathrm{V}_{S}$ and layer thickness were provided. Density was also allowed to vary over a wide range, but did not have significant effects on the inversions as it was allowed to vary in the model space, independently of $\mathrm{V}_{S}$. The parameterisation is the same for the whole region as there are not significant spatial differences in the sedimentary cover or the basement rock (Mortimer, 2004; Browne et al., 2012).

Before the dispersion curves are inverted, they are converted to slowness vs. frequency and resampled to have 50 points in log-log space, so that each period has equal weighting in the inversion and steps resulting from the interpolation between tomography models are smoothed, allowing for better model fits to the data.

At each location, inversions were run several times and compared, to ensure that the maximum possible amount of the parameter space is searched for models. Each inversion performs 50000 iterations and a preferred model calculated from a weighted average of all models below a misfit threshold. For single mode inversions this was set to a misfit below 0.05 .

Table 3.4: Canterbury shear-wave inversion initial model parameterisation

\begin{tabular}{cccc}
\hline $\mathrm{V}_{P}\left(\mathrm{~km} . \mathrm{s}^{-1}\right)$ & $\mathrm{V}_{S}\left(\mathrm{~km} . \mathrm{s}^{-1}\right)$ & Depth to layer base $(\mathrm{km})$ & Density $\left(\mathrm{kg} . \mathrm{cm}^{3}\right)$ \\
\hline $1.0-5.0$ & $1.0-2.5$ & $0.1-3.0$ & $2.0-4.0$ \\
$1.0-7.0$ & $1.9-3.5$ & $2.0-12.0$ & $2.0-4.0$ \\
$4.0-9.2$ & $2.5-4.0$ & - & $2.0-4.0$ \\
\hline
\end{tabular}




\subsection{Results}

The following section presents the tomographic inversions of dispersion curves as a series of 2-dimensional slices at periods between $5-10 \mathrm{~s}$. Each period map samples a depth range, which is approximated through surface wave sensitivity kernels, and this will be examined further in Section 3.4.3.

\subsubsection{Rayleigh group and phase velocity maps}

The tomographic models of Rayleigh wave velocities as a function of period are calculated as described in Section 3.3.4 and presented in Figures 3.22 to 3.25. The group velocity dispersion curves from both vertical ZZ (Figure 3.22) and horizontal RR (Figure 3.23) cross-correlation functions were separately inverted, and similarly for phase velocities (Figures 3.24 and 3.25), in order to compare how well Rayleigh wave velocities are resolved. As expected, the Rayleigh wave velocities increase with increasing period, and phase velocities are consistently higher than group velocities (Stein and Wysession, 2003).

At all periods group velocities decrease towards the north and west of the region, with faster velocities shown to the south of the Greendale Fault and towards Banks Peninsula. Group velocities computed from ZZ correlations are slightly lower than those of RR correlations. At $5 \mathrm{~s}$ period, to the northeast of the region velocities are approximately $1.75-2.0 \mathrm{~km} . \mathrm{s}^{-1}$ for ZZ (Figure 3.22 ) compared to $2.0-2.2 \mathrm{~km} . \mathrm{s}^{-1}$ for RR (Figure 3.23). At longer periods of $8-10 \mathrm{~s}$ the models are in agreement, apart from a slightly higher velocity zone up to $3.0 \mathrm{~km} . \mathrm{s}^{-1}$ for the RR models.

Rayleigh phase velocities for periods $5-8 \mathrm{~s}$ are shown in figures 3.24 and 3.25. It was not possible to model to longer periods as the path coverage was not sufficient to give good spatial resolution. Seismic velocities for both sets of correlations are consistent, with Rayleigh phase velocities ranging from $2.5 \mathrm{~km} . \mathrm{s}^{-1}$ at $5 \mathrm{~s}$ to 3.1 $\mathrm{km} . \mathrm{s}^{-1}$ at $8 \mathrm{~s}$. Similar to group velocities, Rayleigh phase speeds decrease to the north and west of the model region. The RR maps (Figure 3.25) show slightly increased velocities to the northeast of the region, for example up to $3.2 \mathrm{~km} . \mathrm{s}^{-1}$ at $8 \mathrm{~s}$, however this is at the edge of the region with best resolution (white contour) and suffers from a lack of seismic stations. 

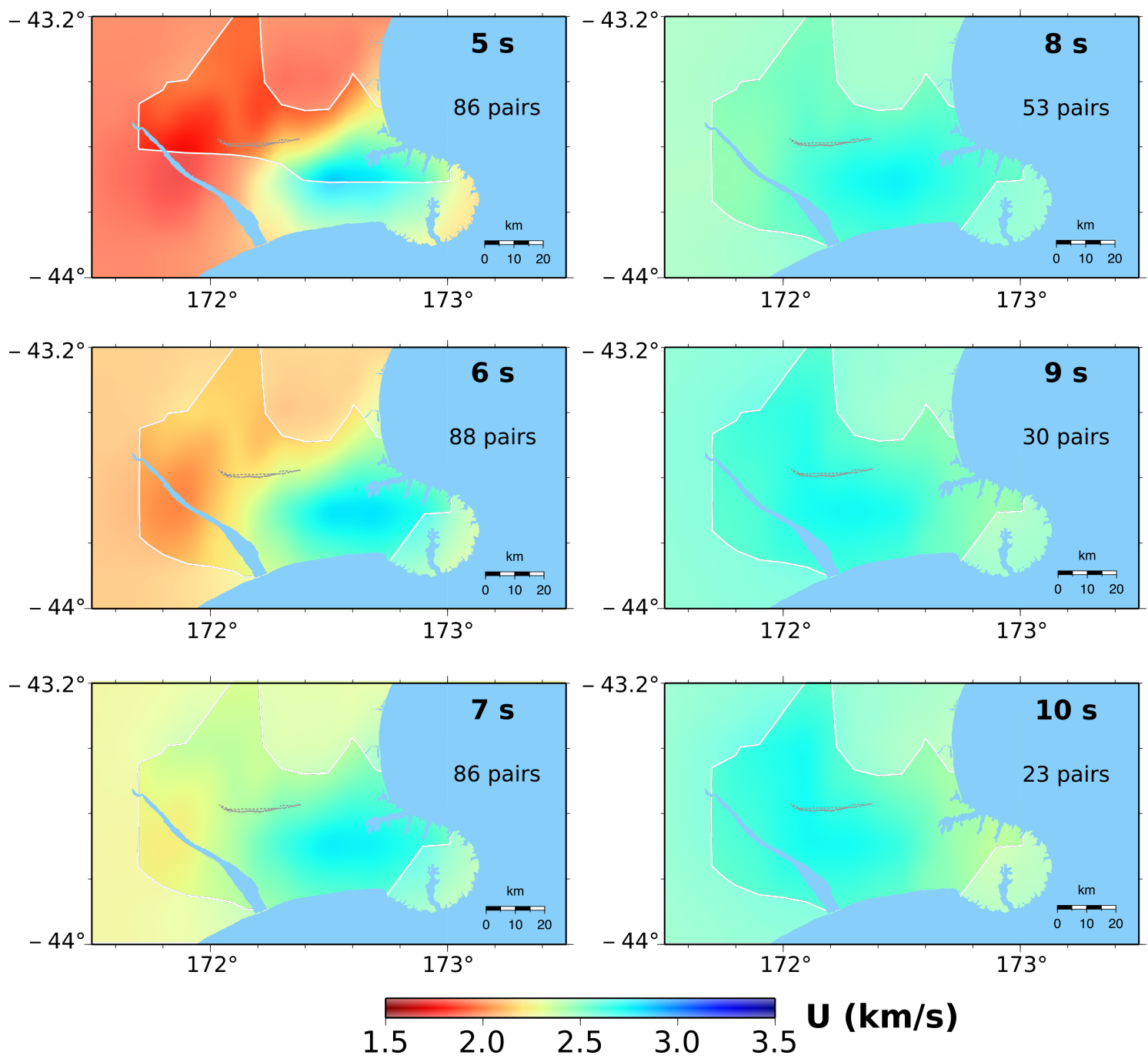

Figure 3.22: Rayleigh wave group velocity maps for 5 to 10 s periods from ZZ crosscorrelations. Bold colours denote the model region where spatial resolution is $40 \mathrm{~km}$ or lower, with white contours outlining this region and colours outside the region masked. The surface expression of the Greendale Fault is indicated by the dashed grey lines. 

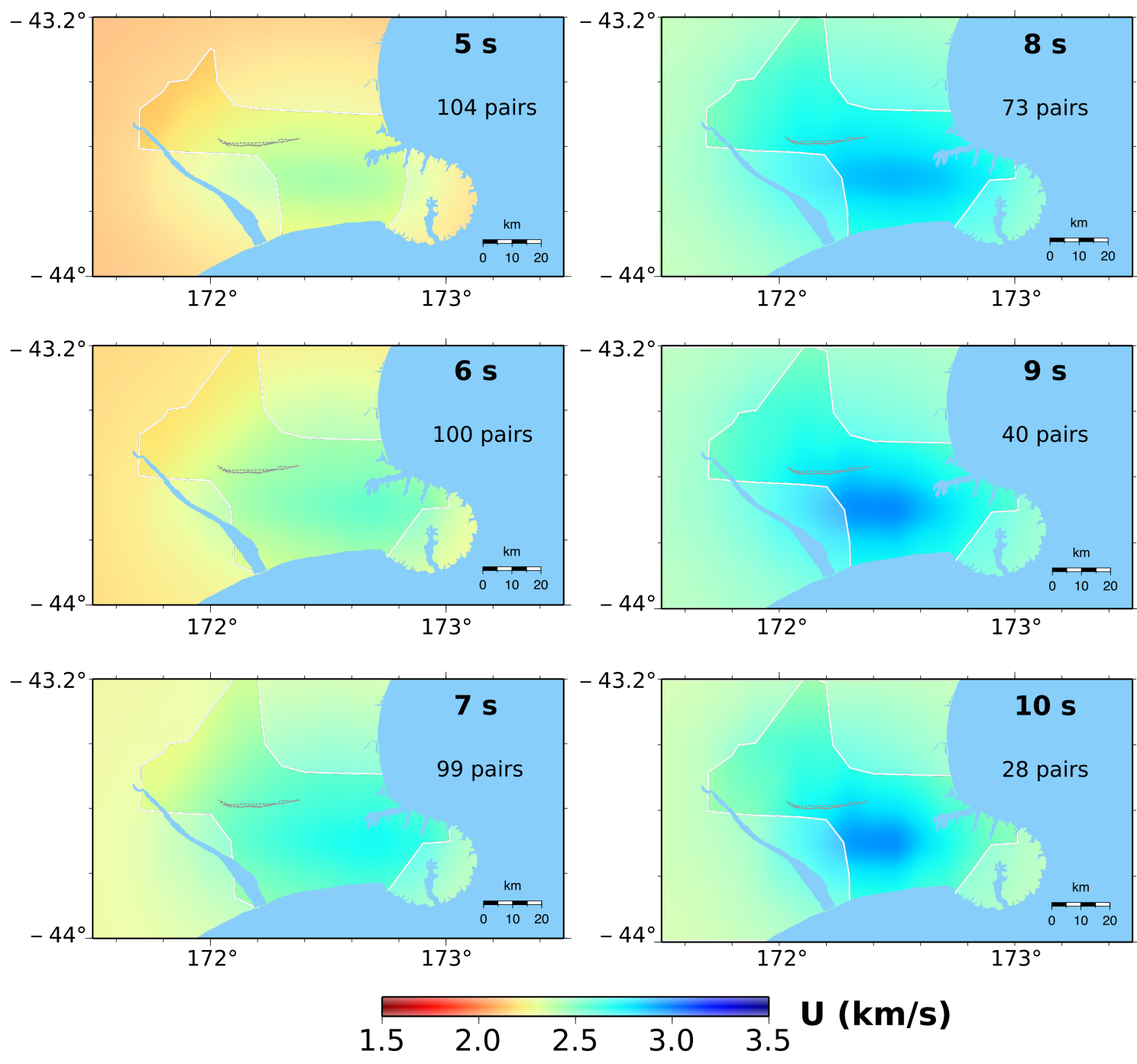

Figure 3.23: Rayleigh wave group velocity maps for 5 to $10 \mathrm{~s}$ periods from RR crosscorrelations. Bold colours denote the model region where spatial resolution is $40 \mathrm{~km}$ or lower, with white contours outlining this region and colours outside the region masked. The surface expression of the Greendale Fault is indicated by the dashed grey lines. 

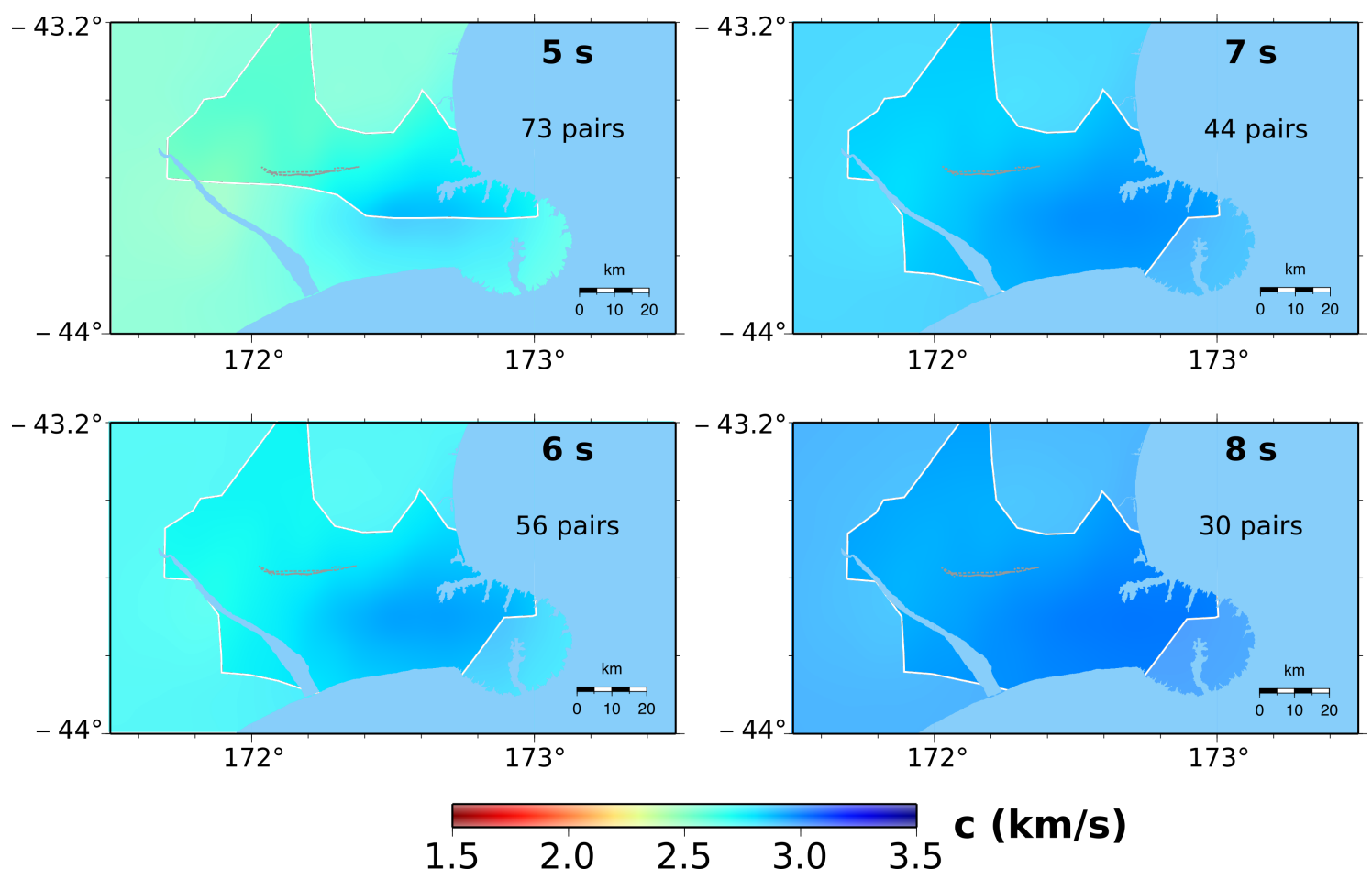

Figure 3.24: Rayleigh wave phase velocity maps for 5 to 8 s periods from ZZ crosscorrelations. Bold colours denote the model region where spatial resolution is $40 \mathrm{~km}$ or lower, with white contours outlining this region and colours outside the region masked. The surface expression of the Greendale Fault is indicated by the dashed grey lines. 

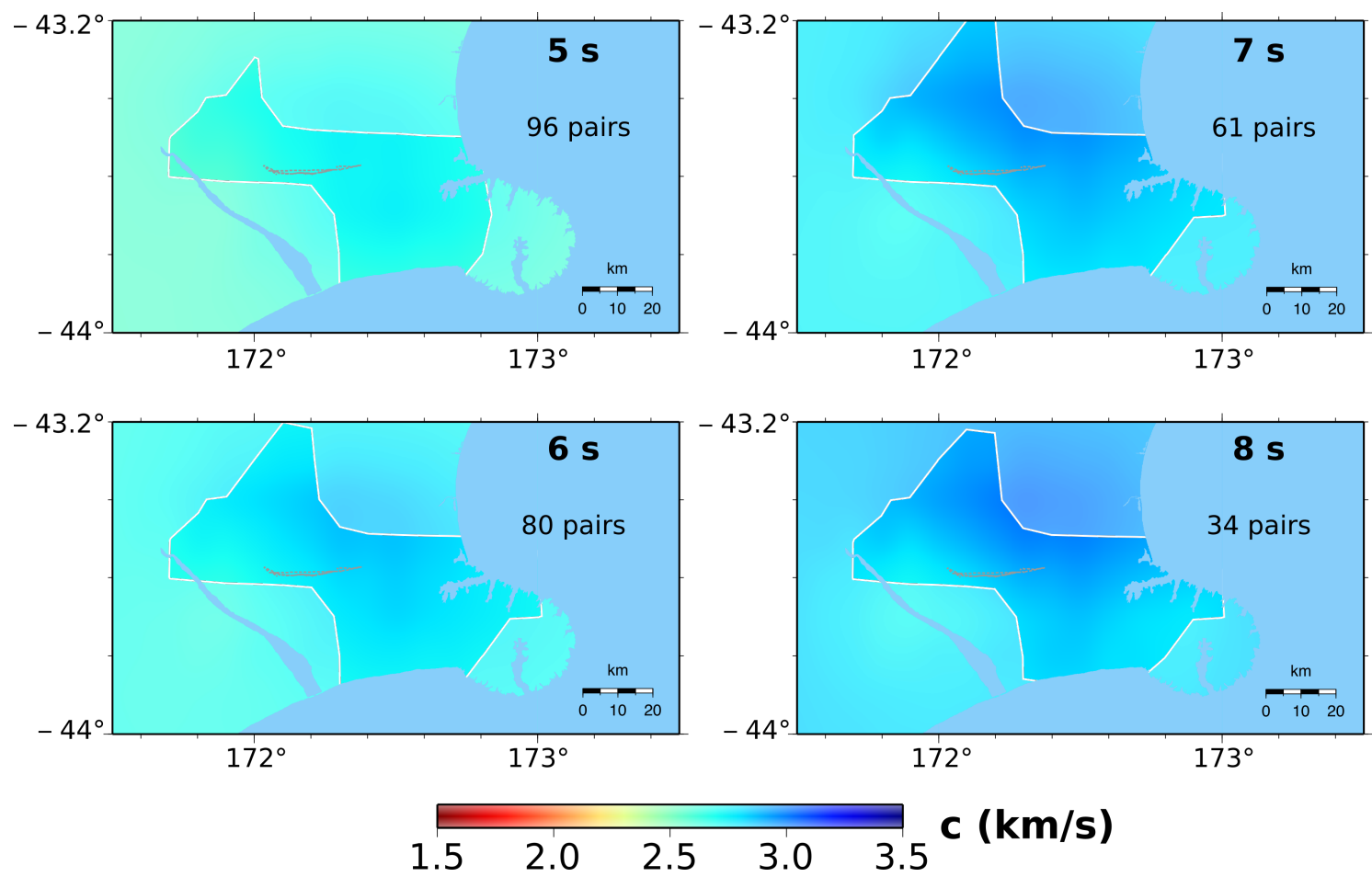

Figure 3.25: Rayleigh wave phase velocity maps for 5 to $10 \mathrm{~s}$ periods from RR crosscorrelations. Bold colours denote the model region where spatial resolution is $40 \mathrm{~km}$ or lower, with white contours outlining this region and colours outside the region masked. The surface expression of the Greendale Fault is indicated by the dashed grey lines. 


\subsubsection{Love group and phase velocity maps}

The region of good spatial resolutions of less that $40 \mathrm{~km}$ is greatly reduced for Love waves compared to Rayleigh. This is due to lower signal-to-noise ratios for TT cross-correlations. This caused far fewer cross-correlations passing the constraints applied prior to tomographic inversions, resulting in a decreased path coverage compared to RR and ZZ correlations. In addition, the fewer available station paths are concentrated in a smaller region surrounding the Greendale Fault.

Velocity maps for Love group and phase are shown in Figures 3.26 and 3.27. Group velocities as low as $1.3 \mathrm{~km} . \mathrm{s}^{-1}$ are observed at $5 \mathrm{~s}$, and remain less than $2.0 \mathrm{~km} . \mathrm{s}^{-1}$ up to $7 \mathrm{~s}$ (note the slightly altered scale in Figure 3.26 to accomodate low velocities). At $5-7 \mathrm{~s}$ there is a high velocity zone to the south of the Greendale Fault, reaching velocities of up to $2.4 \mathrm{~km} . \mathrm{s}^{-1}$. This feature is not present at $8 \mathrm{~s}$ however, with consistent velocities of $2.2-2.3 \mathrm{~km} . \mathrm{s}^{-1}$. Phase velocities are near constant throughout the region, ranging from $2.2 \mathrm{~km} . \mathrm{s}^{-1}$ at $5 \mathrm{~s}$ to $2.7 \mathrm{~km} . \mathrm{s}^{-1}$ at $7 \mathrm{~s}$. Some patches of slightly increased velocities are seen at the edges of the region of high model resolution and so are likely insignificant and artefacts of the inversion.
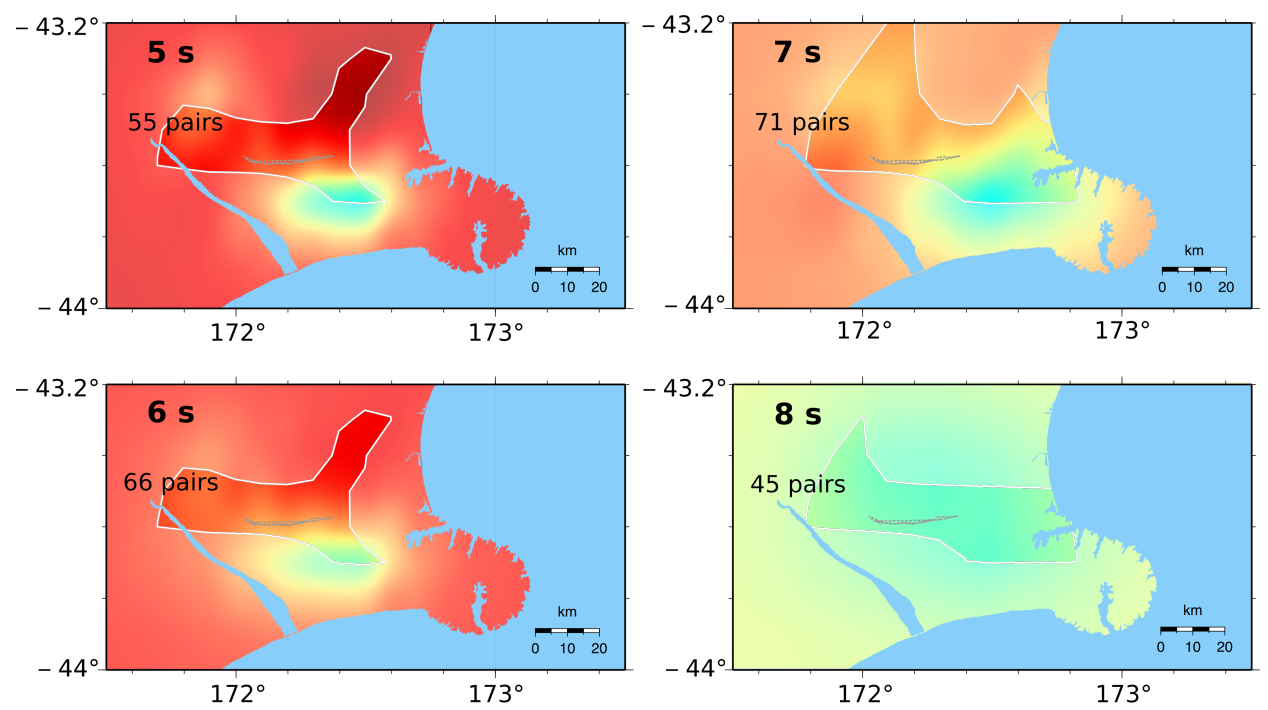

$\begin{array}{lllllll}1.0 & 1.5 & 2.0 & 2.5 & 3.0 & 3.5\end{array}$

Figure 3.26: Love wave group velocity maps for 5 to $10 \mathrm{~s}$ periods from TT crosscorrelations. Bold colours denote the model region where spatial resolution is $40 \mathrm{~km}$ or lower, with white contours outlining this region and colours outside the region masked. The surface expression of the Greendale Fault is indicated by the dashed grey lines. 

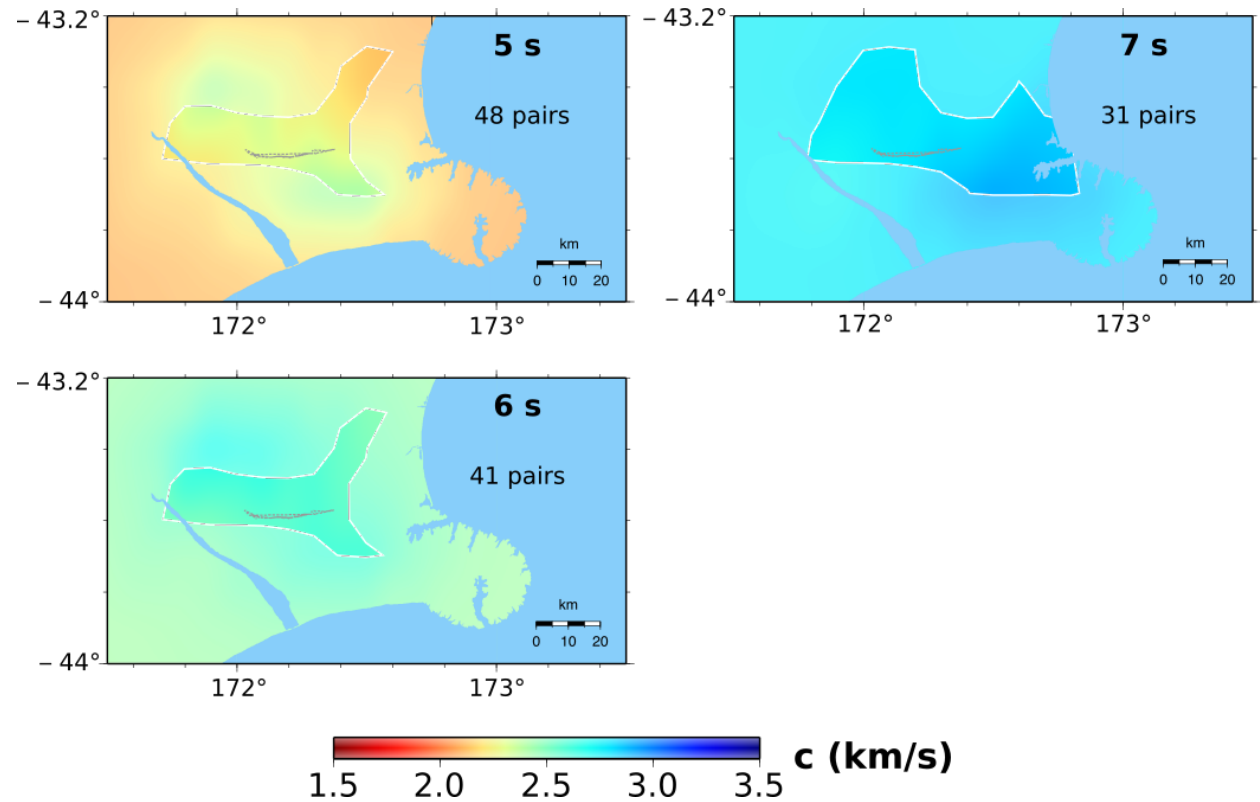

Figure 3.27: Love wave phase velocity maps for 5 to $7 \mathrm{~s}$ periods from TT crosscorrelations. Bold colours denote the model region where spatial resolution is $40 \mathrm{~km}$ or lower, with white contours outlining this region and colours outside the region masked. The surface expression of the Greendale Fault is indicated by the dashed grey lines.

\subsubsection{Surface wave sensitivity kernels}

The examination of surface wave sensitivity kernels can give an indication of the approximate depths that Rayleigh and Love waves are most sensitive to, or in other words, sampling, as a function of period, as explained in Section 2.1.1. Sensitivity kernels were produced for the Canterbury Plains, using an average regional model calculated from the models of Eberhart-Phillips et al. (2010) and additionally including a shallow low velocity layer (see Appendix B for the 1-D model used). Figure 3.28 illustrates the kernels for both Rayleigh and Love (top and bottom panels), group and phase velocities (right and left panels). Curves are shown for $5-10 \mathrm{~s}$ for each surface wave phase. Group velocities have higher sensitivities compared to phase velocities, however phase velocities are sensitive over greater depth ranges and penetrate deeper than group velocities. The shallow sensitivity for all surface wave phases decreases with increasing period. At depths below $8 \mathrm{~km}$, longer periods (e.g. 10 s) have higher sensitivity than short periods.

A clear feature of the curves is that Love velocities are highly sensitive to the shallow subsurface, whereas Rayleigh waves sample deeper, to $14 \mathrm{~km}$ for $10 \mathrm{~s}$ period. The Rayleigh group and phase velocities are mostly sensitive to $3-6 \mathrm{~km}$ depth, but still have sensitivity deeper and in the uppermost $500 \mathrm{~m}$ to $1 \mathrm{~km}$. Love waves are highly sensitive to the uppermost $2 \mathrm{~km}$, with a second lesser peak at depths of $3-6 \mathrm{~km}$ as 
for Rayleigh waves.

The slower velocities for Love compared to Rayleigh waves observed for the short periods, in particular 5 and $6 \mathrm{~s}$, can be explained by the fact that the Love waves are mostly excited at much shallower depths below $2 \mathrm{~km}$ for shorter periods.

The high velocity region south of the Greendale Fault is approximately $25-30 \mathrm{~km}$ in diameter. There are stations that are situated here, CCH04 and MQZ, however it lies in a region where the spatial resolution is over $34 \mathrm{~km}$ and so is likely not a real feature but an artefact of the inversion process, caused by higher velocities from correlations from station BANK1, which is situated above the volcanic rocks of Banks Peninsula.

\subsubsection{1-D shear velocity models}

Results of 1-dimensional shear velocity inversions are presented in the following section. Higher confidence can be placed in the phase velocity dispersion measurements as they had far less variation in the month-long cross-correlations, and therefore uncertainties in the overall stacked dispersion measurements are lower. Additionally, cross-correlations of the radial-radial component had higher signal-to-noise ratios than vertical components, resulting in a denser path coverage in tomographic models. For these reasons inversions for shear velocity models were performed on phase velocities of RR-derived Rayleigh and TT-derived Love dispersion curves. Figure 3.29 shows an example of shear-wave inversion models from Rayleigh and Love dispersion curves, at point C, situated centrally on the Greendale Fault (for location see map inset in Figure 3.29). Grey lines are models with misfits higher than the accepted threshold of 0.05 and the solid black line is the preferred model, calculated as an average of accepted models, weighted by misfit. Both models are in agreement for the uppermost $4 \mathrm{~km}$, with a $500 \mathrm{~m}$ thick low velocity layer of 1.0 - $1.2 \mathrm{~km} . \mathrm{s}^{-1}$ overlying a layer with a velocity of $2.6 \mathrm{~km} . \mathrm{s}^{-1}$. Discrepancies arise below $4 \mathrm{~km}$, as models derived from Rayleigh phase measurements require this layer to extend to $7 \mathrm{~km}$ depth, compared to $4 \mathrm{~km}$ for the Love wave inversion. The most likely reason for this is that the input Love phase dispersion curves have measurements within a narrower period band of $4-8 \mathrm{~s}$, compared to the $4-10 \mathrm{~s}$ of Rayleigh phase measurements. This was due to Love dispersion curves having far larger uncertainties at longer periods, and so measurements over $8 \mathrm{~s}$ were removed from subsequent analyses. In addition, as explained previously, the sensitivity of Love waves is concentrated in the uppermost $6 \mathrm{~km}$ of the subsurface, so realistic $\mathrm{V}_{S}$ inversion measurements cannot be obtained below this depth. Joint inversions of Love and Rayleigh waves were attempted, however resulting models were not ac- 
ceptable as they were weighted towards Rayleigh waves too highly. Rayleigh waves have higher sensitivity at periods and depths compared to Love waves, which may explain this.

Figure 3.30 presents 1-D $V_{S}$ models for several locations throughout the region. Points A and B, located to the northern edge of the Canterbury plains, and towards the foothills of the Southern Alps, have a low velocity layer at the immediate subsurface, with $\mathrm{V}_{S}=1.2-1.6 \mathrm{~km} \cdot \mathrm{s}^{-1}$, to a depth of $1 \mathrm{~km}$ at $\mathrm{A}$ and $1.6 \mathrm{~km}$ at B. They consist of a $7-8 \mathrm{~km}$ thick layer above with velocity $2.5-2.7 \mathrm{~km} . \mathrm{s}^{-1}$ which increases to $3.2-3.4 \mathrm{~km} . \mathrm{s}^{-1}$ below the boundary. A thin and slow $500 \mathrm{~m}$ thick subsurface layer exists at point $\mathrm{C}$, at the midpoint of the Greendale Fault. Below this the model shows the same velocities as A and B to the northwest. Point D, at the northern edge of Banks Peninsula consists of a $3 \mathrm{~km}$ thick layer with velocity $1.7-2.1 \mathrm{~km} . \mathrm{s}^{-1}$ overlying a layer with shear velocity $3.4-3.8 \mathrm{~km} . \mathrm{s}^{-1}$. This model and nearby locations were the least constrained of all, as they are located to the southeastern extent of the model area, and located where spatial resolution is lowest.

\subsection{Discussion}

The results from the $\mathrm{V}_{S}$ 1-D inversions show trends and features which can be attributed to the geology and structures of the Canterbury Plains. The separate inversions of Rayleigh and Love waves show a thinning of the uppermost layer from over $1.6 \mathrm{~km}$ thick to $1.0 \mathrm{~km}$ towards the edges of the Canterbury Plains. This layer is sedimentary cover decreasing in thickness towards the foothills of the Southern Alps, in agreement with regional geological maps (Browne et al., 2012; Ghisetti and Sibson, 2012) showing sedimentary cover of sandstone, limestone and mudstone successions overlying basement rocks of greywacke. This observation is consistent with Syracuse et al. (2013) who observed increased shear velocities at all depths to the northwest of the Plains.

The Darfield Fault exhibits a thin, $500 \mathrm{~m}$ thick, subsurface layer with greatly decreased velocities, as low as $1.0 \mathrm{~km} . \mathrm{s}^{-1}$. This layer is present at all locations for the length of the surface expression of the fault, and extending up to $30 \mathrm{~km}$ further east, towards the city of Christchurch (Figure not shown). It exists in models inverted from the results of tomographic inversions and from dispersion curves obtained directly from cross-correlation functions, allowing high confidence that this feature is a real observation. It is likely to be caused by a highly damaged zone in the immediate vicinity of the fault where sediments have been disrupted by the 
Darfield earthquakes and the aftershocks. Reyners et al. (2014) observed a well constrained region of low $\mathrm{V}_{P} / \mathrm{V}_{S}$ ratios following the Darfield earthquake and its location matches well with the low damage layer observed in this study. The result is also in agreement with the depth extent of the temporal velocity changes of Chapter 4 , where velocities were decreased in the uppermost $2 \mathrm{~km}$ due to cracking. This feature is not present in the models of Syracuse et al. (2013), however the depth resolution of their models is not as fine as here.

The boundary of the deepest layer of our inversions rapidly decreases in depth in the southwest of the region. The tomography models additionally show increased velocities in the area at long periods. Syracuse et al. (2013) found velocities increased up to $48 \%$ here, and interpreted this as an extension of the volcanic rocks of the Port Hills and Banks Peninsula (Browne et al., 2012). It is not possible however, to confidently interpret the models of this study as such. It has previously briefly been touched upon in Section 3.3.4, that in this area there is decreased path coverage and spatial resolution. There is only one seismic station located nearby; BANK1, and the volcanics of Banks Peninsula are likely to be biasing the velocities measured on paths crossing this area from BANK1.

Discrepancies between the 1-D $\mathrm{V}_{S}$ inversions of different cross-correlation components were observed for many locations throughout the region. This is attributed to differing resolutions in the tomography models and the sensitivity of surface waves to shear waves at depth. Love group and phase velocity measurements could not be reliably obtained for periods longer than $8 \mathrm{~s}$, and so the depth extent of the models is not as great. Signal-to-noise ratios were highest for radial-radial correlation functions, and resulted in more station pairs available for tomographic inversions than for other cross-correlation components. This mostly affects the size of the region with resolutions better than $40 \mathrm{~km}$ due to an increased number of long path-length pairs. The calculated resolutions within the model regions were still good for the other components, with the majority of the model space having a minimum possible size of resolvable features of $\sim 22 \mathrm{~km}$.

A final point of discussion is the lack of higher modes in the cross-correlation functions. As shown in the synthetic Green's functions (Section 3.3.2), first higher-mode Rayleigh waves could only be generated where a thin low velocity layer near the surface was present. The contrast between this layer and the one beneath it was required to be very sharp and large. Using a smoother multi-layer velocity model of Eberhart-Phillips et al. (2010) produced only fundamental mode Rayleigh waves for both ZZ and RR cross-correlations. The $\mathrm{V}_{S}$ inversions showed this high velocity contrast only in the vicinity of the Greendale Fault, however for these station pairs only fundamental mode Rayleigh waves were observed with low velocities (Figure 
3.18). It is possible that higher-modes were generated for the on-fault station paths, however the AFTAN procedure picks the highest amplitude arrivals, and so they would not have had enough energy to be selected over fundamental-mode Rayleigh waves. 

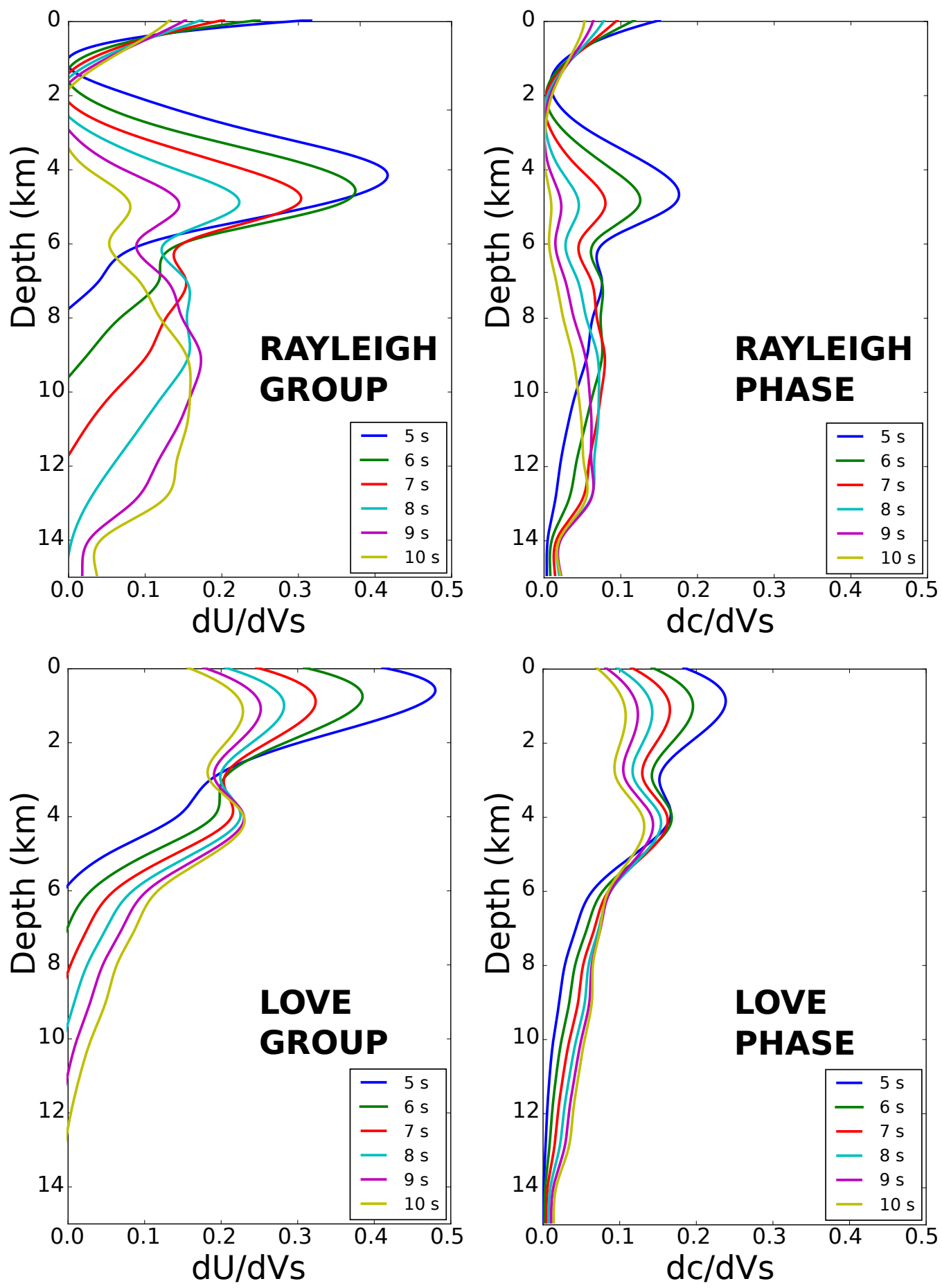

Figure 3.28: Sensitivity kernels of fundamental mode surface waves with respect to shear velocity as a function of depth for 5-10 s. An average regional velocity model of Eberhart-Phillips et al. (2010) was used to compute partial derivatives (see Appendix B) for Rayleigh (top panels) and Love (lower panels), group and phase velocities (left and right panels respectively. 

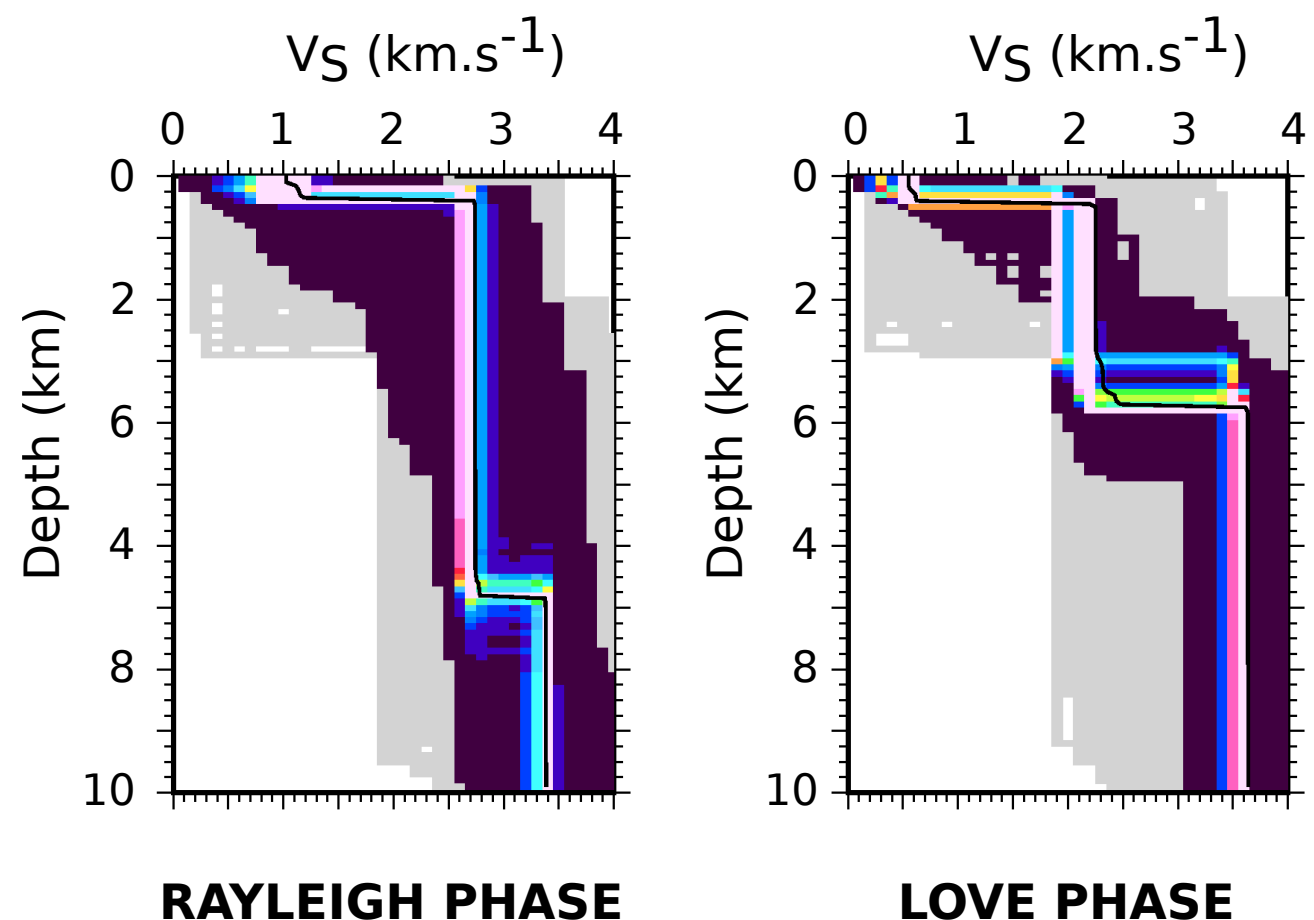

RAYLEIGH PHASE

\section{LOVE PHASE}

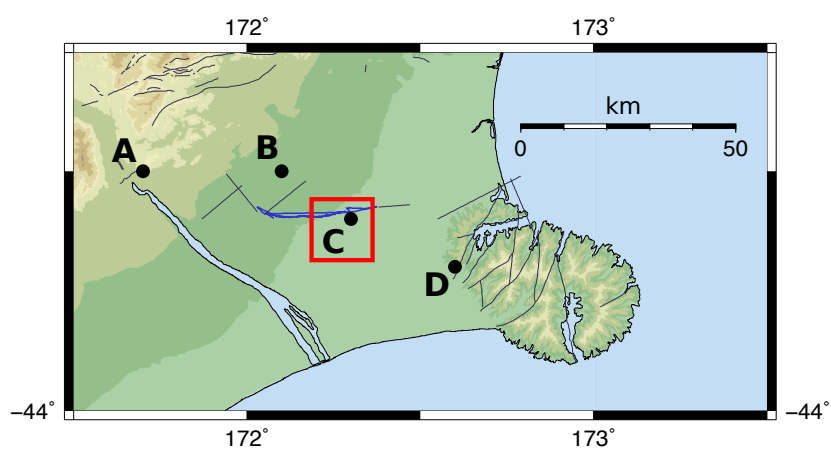

Figure 3.29: One-dimensional $\mathrm{V}_{S}$ models for Point $\mathrm{C}$, located at -43.6, 172.3 (see map inset). Inversions from Rayleigh phase velocity (left) and Love phase velocity (right) dispersion curves are presented separately. The preferred model, from all models with misfits below 0.05 , is shown as a black line, with coloured models increasing in warmth with decreasing misfit. Models in grey are those that were disregarded due to high model misfit values. 

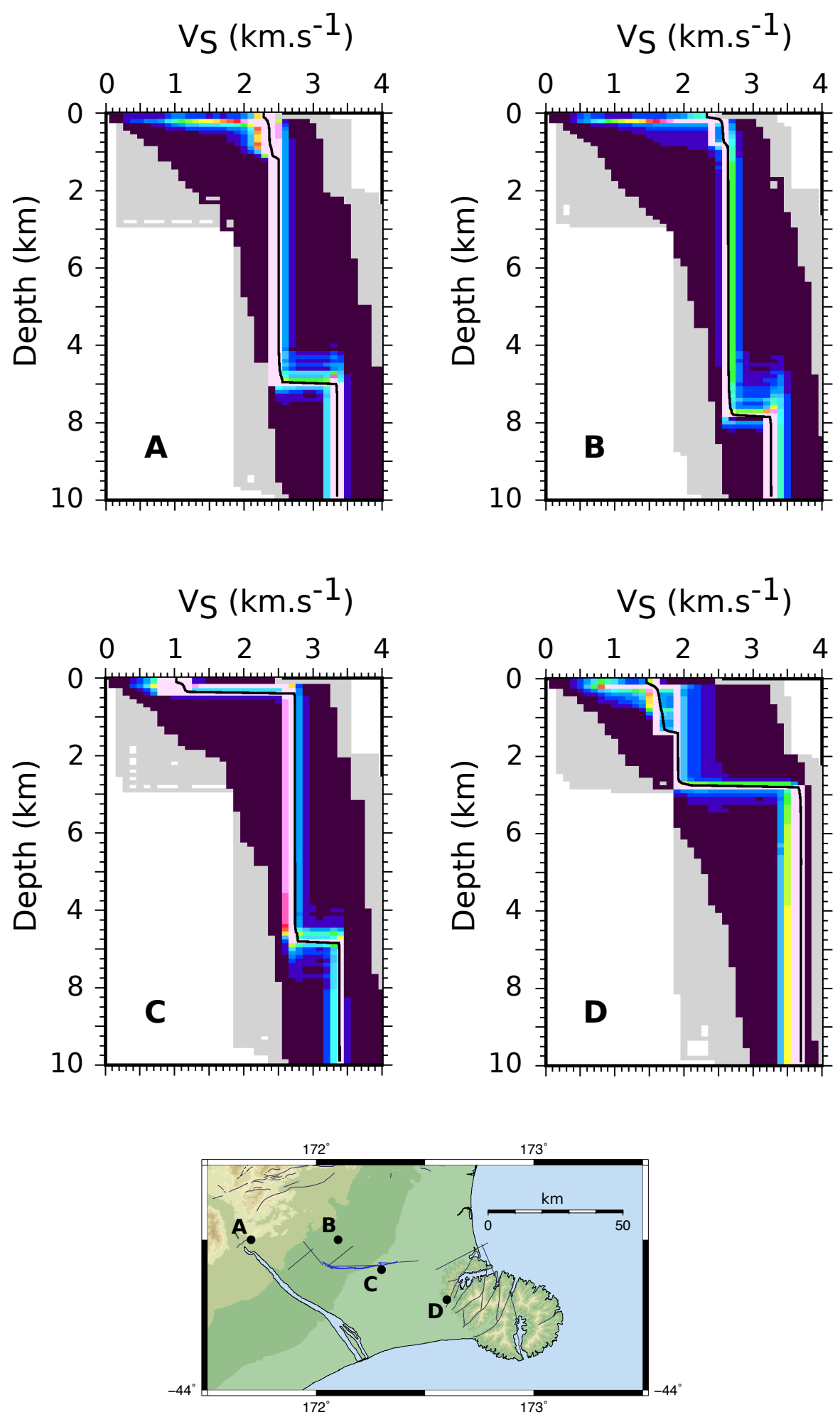

Figure 3.30: One-dimensional $\mathrm{V}_{S}$ models for Canterbury from Rayleigh phase velocity measurements. For locations A - D (see map inset) inversions from Rayleigh phase velocity dispersion curves are presented. The preferred model, from all models with misfits below 0.05, is shown as a black line, with coloured models increasing in warmth with decreasing misfit. Models in grey are those that were disregarded due to high model misfit values. 


\section{Investigation into Time-Varying Proper- ties of Surface Waves and Noise Charac- teristics in Canterbury}

\subsection{Abstract}

Quantifying seismic velocity changes following large earthquakes can provide insights into fault healing and reloading processes. This study presents temporal velocity changes detected following the September $2010 \mathrm{M}_{w}$ 7.1 Darfield event in Canterbury, New Zealand. We use continuous waveform data from several temporary seismic networks lying on and surrounding the Greendale Fault, with a maximum inter-station distance of $156 \mathrm{~km}$. Nine-component, day-long Green's functions were computed for frequencies between 0.1 and $1.0 \mathrm{~Hz}$ for continuous seismic records from immediately after the 4 September 2010 earthquake until 10 January 2011. Using the movingwindow cross-spectral method seismic velocity changes were calculated. Over the study period, an increase in seismic velocity of $0.14 \% \pm 0.04 \%$ was determined near the Greendale Fault, providing a new constraint on postseismic relaxation rates in the region. A depth analysis further showed that velocity changes were confined to the uppermost $5 \mathrm{~km}$ of the subsurface. We attribute the observed changes to postseismic relaxation via crack-healing of the Greendale Fault and throughout the surrounding region.

\subsection{Introduction}

When considering hazards in earthquake-prone regions, knowledge of subsurface material properties is important. The monitoring of crustal properties, such as seismic surface wave velocities can elucidate valuable information about the regional stresses. Ambient seismic noise is increasingly being used to evaluate crustal seismic properties. Cross-correlations of long-duration seismic records yield information 
about the propagation velocities of scattered surface waves (Shapiro and Campillo, 2004). For a pair of seismic stations, the cross-correlation functions contain positive and negative time lags, which are analogous to the causal and acausal Green's functions (Bensen et al., 2007; Yang et al., 2008). That is, the positive time lags represent arrival times of waves travelling in one direction as if one station is a source and the other the receiver. Negative time lags represent waves travelling in opposite directions, with roles reversed.

A method for detecting seismic velocity variations, known as Moving-Window CrossSpectral (MWCS) analysis, using earthquake multiplets, was proposed by Ratdomopurbo and Poupinet (1995) and has been modified for ambient seismic noise (Clarke et al., 2011; Lecocq et al., 2014). Relative delay times between long-duration cross-correlation stacks and those of shorter duration are computed and can be used to calculate relative velocity changes. This technique has since been used to successfully detect seismic velocity variations following large earthquakes. Brenguier et al. (2008) first used the MWCS method to examine earthquake-induced seismic velocity changes with ambient noise cross-correlations. A $0.08 \%$ increase in velocity was recorded over three years following the $\mathrm{M}_{w}$ 6.0 Parkfield earthquake in California and velocities returned to pre-seismic levels at the same rate as GPS displacements over several years.

The use of ambient seismic noise in New Zealand is already well established. The first major study into the ambient seismic noise field in New Zealand produced Rayleigh wave group velocity maps, and highlighted the Canterbury basin as a lowvelocity region (Lin et al., 2007). Smaller-scale studies considered several regions of New Zealand's North Island. Behr et al. (2010) estimated a Moho depth of $28 \mathrm{~km}$ for the Northland Peninsula from surface wave dispersion curves, in agreement with active-source methods. Beamforming analyses of the ambient noise field in New Zealand highlighted the first higher-mode Rayleigh waves (Brooks et al., 2009) and suggested several possible sources concerning ocean-wave movements (Behr et al., 2013).

The $\mathrm{M}_{w}$ 7.1 Darfield earthquake of 4 September 2010 was the first and largest event in a damaging earthquake sequence that struck the Canterbury region in the South Island of New Zealand between 2010 and 2012. The earthquake occurred west of Christchurch on the previously unrecognised Greendale Fault (Bannister and Gledhill, 2012). A surface rupture of nearly $30 \mathrm{~km}$ was observed, exhibiting predominantly right-lateral strike-slip motion (Quigley et al., 2012). The aftershocks that followed revealed a broad pattern of eastwards hypocentral migration and included several earthquakes larger than $\mathrm{M}_{L} 5$ (Syracuse et al., 2012, 2013). This sequence included the $\mathrm{M}_{w} 6.3$ Christchurch earthquake of 22 February 2011, which struck 
at a depth of $3-4 \mathrm{~km}$ (Kaiser et al., 2012), $6 \mathrm{~km}$ south of the central business district (Bannister and Gledhill, 2012), causing widespread damage throughout the city, resulting in 185 deaths. Recorded ground accelerations of over $1.25 \mathrm{~g}$ during the Darfield earthquake were the largest recorded in New Zealand (Fry and Gerstenberger, 2011) to that point and apparent stresses of almost $16 \mathrm{MPa}$ are extremely high compared to global averages (Fry and Gerstenberger, 2011). Fry et al. (2014) used ambient seismic noise on the permanent GeoNet network to measure anisotropy from surface wave dispersion of fundamental mode Rayleigh waves throughout Canterbury. At upper crustal depths, east-west trending fast axes parallel to the Greendale Fault and Cretaceous faults were recorded. At lower-crustal depths, fast axes were shown to be parallel to the present plate boundary strain direction (Fry et al., 2014). Ambient noise cross-correlations within the region contain higher-mode signals (Savage et al., 2013) and comparisons of horizontal and vertical component correlation functions show strong first-higher-mode Rayleigh waves on paths parallel to nearby ocean wave directions. A basement resonance frequency of approximately $0.4 \mathrm{~Hz}$ was obtained from $\mathrm{H} / \mathrm{V}$ ratios of higher-mode Rayleigh waves (Savage et al., 2013).

Several studies have focussed on observing the co- and post-seismic responses of Canterbury. Beavan et al. (2012) observed post-seismic deformation following the Darfield earthquake using continuous GPS measurements and detected deformation to the east of the Darfield epicentre, close to the epicentre of the subsequent Christchurch earthquake. In addition, several distinct fault segments of the Greendale Fault were delineated. These findings were supported by Syracuse et al. (2012, 2013), who relocated aftershock hypocentres and computed focal mechanisms to highlight eight fault segments. Inversions of focal mechanisms and shear wavesplitting analysis yields an average fast direction of $116 \pm 18^{\circ}$ (Holt et al., 2013), similar to the azimuth of maximum horizontal compressive stress (Townend et al., 2012). Some stations gave fast directions subparallel to the Greendale Fault, suggesting either structure dependent anisotropy, or stress changing near the fault Holt et al. (2013). Inversions of stress from focal mechanisms showed even clearer rotations of stress near the fault trace compared to those further from the trace. Assuming the rotations along the fault were caused by rotation due to the earthquake stress drop, Holt et al. (2013) inferred that $40 \%$ of preseismic differential stress on the Greendale Fault was released in the Darfield earthquake. A high coseismic stress drop was reported (Quigley et al., 2012; Beavan et al., 2012). Reyners et al. (2014) measured low seismic P- to S-wave ratios of 1.60 post-seismically, decreasing from 1.71 prior to the earthquake. They interpreted the cause to be weakened greywacke producing fault-zone cracking. Reyners et al. (2014) further suggested that the long 
delay between the Darfield earthquake and the later Christchurch event was a result of recovering rock strength through crack-healing. Sustained hydrological effects in river discharge and groundwater levels were observed over an interval of a year after the earthquake, with the majority of recovery in the hours immediately following the event (Cox et al., 2012).

We report here on temporal velocity changes detected in the four months following the Darfield earthquake. Several temporary datasets were analysed in order to examine the short-term response of the seismic velocities in the Canterbury region.

\subsection{Data and Methods}

The data used in this study were acquired using two temporary, rapid-response networks deployed across the Canterbury region following the Darfield event to record the aftershock sequence (Figure 4.1, top panel). The GeoNet rapid-response network consisting of nine short-period sensors was operational from 5 September 2010 to 29 September 2010 (Gledhill et al., 2011). Thirteen seismometer stations were deployed by Victoria University of Wellington (VUW), the University of Wisconsin-Madison (UWM) and the University of Auckland (UA) (Syracuse et al., 2012; Savage et al., 2013). These broadband and short-period seismometers were in operation for four months from 18 September 2010 until 13 January 2011. The two temporary networks consisted of stations deployed on and surrounding the Greendale Fault. Interstation paths ranged from $6 \mathrm{~km}$ to $156 \mathrm{~km}$ (Figure4.1, top panel). The two datasets were processed separately and augmented by data from several nearby permanent broadband stations of the national GeoNet network (Petersen et al., 2011).

Daily cross-correlation functions were computed following the methodology of Bensen et al. (2007) using the MSNoise package (Lecocq et al., 2014) for the duration of both temporary deployments. Several pre-processing steps enhanced the data for ambient noise analyses. Day-long vertical and horizontal component seismograms were resampled to $25 \mathrm{~Hz}$ and a $0.01-12 \mathrm{~Hz}$ bandpass filter was applied. The mean, trend and instrument responses were removed to allow cross-correlations across different networks, as several different instrument types were used. Spectral whitening between 0.05 and $10.0 \mathrm{~Hz}$ was applied to all daily traces, prior to one-bit normalisation, suppressing higher-amplitude signals from aftershocks and enhancing scattered waves. Although several methods of amplitude normalisation exist (Bensen et al., 2007), one-bit normalisation is employed here as it is most robust in removing signals from aftershocks of all magnitudes, which dominate the records in the this time period. For each pair of stations, the radial $(\mathrm{R})$, transverse $(\mathrm{T})$ and vertical $(\mathrm{Z})$ 

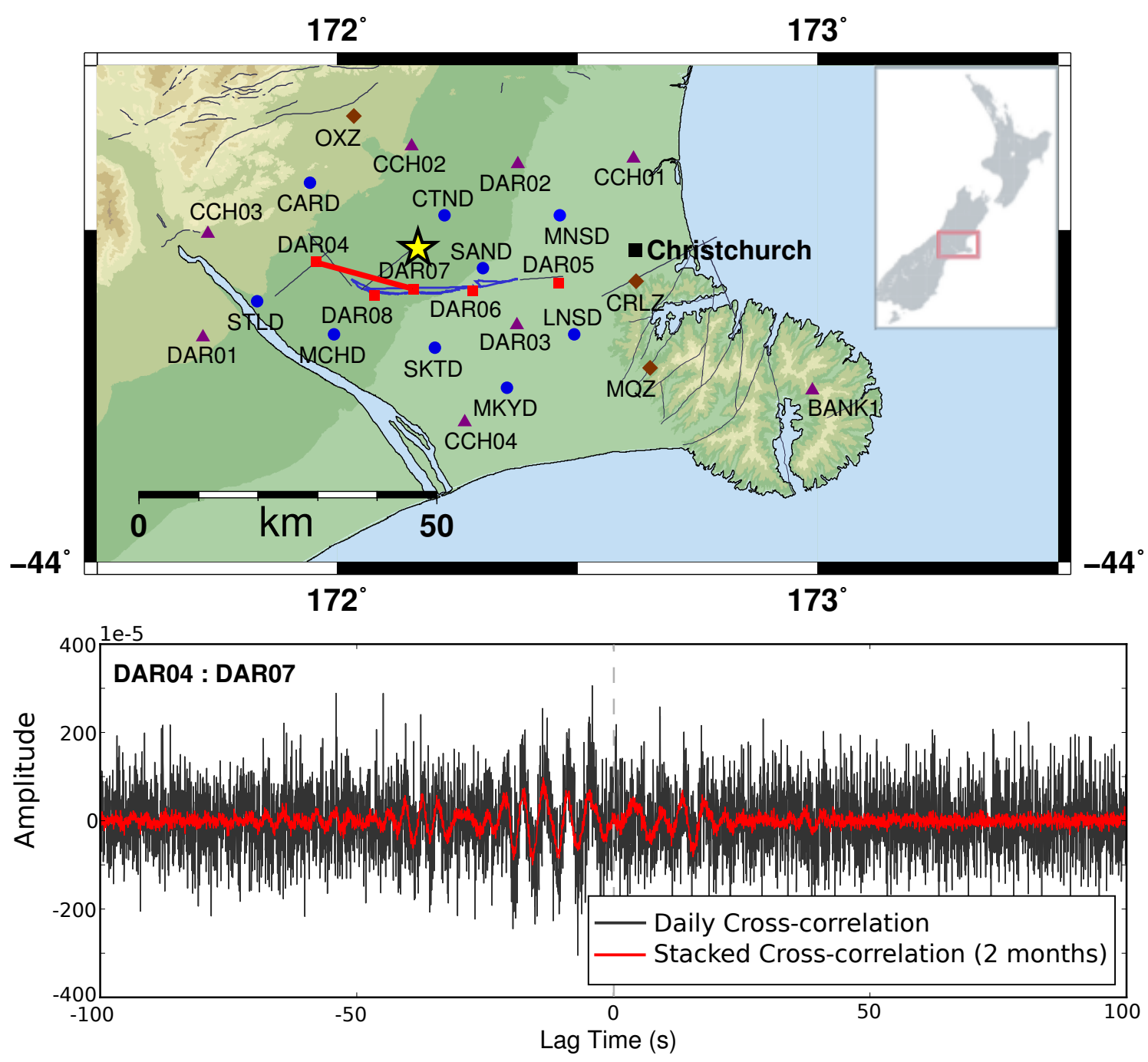

Figure 4.1: Top panel: Map of Canterbury area showing the epicentre of the Darfield earthquake (yellow star). Mapped faults are shown by thin grey lines and the Greendale Fault (Quigley et al., 2012) is highlighted in blue. Coloured symbols show locations of GeoNet permanent broadband stations (brown diamonds), GeoNet rapid-response temporary stations (blue circles), and VUW-UWM-UA (red squares and purple triangles for on- and off-fault stations, respectively). Inset shows the location of the study area within New Zealand. Bottom panel: Effect of stacking on cross-correlation functions. The example shown is for the vertical-vertical correlation function for on-fault station pair DAR04-DAR07 (path highlighted on top panel). The black trace is an individual daily correlation for 19 November 2010 and the red line is the corresponding average stack. 


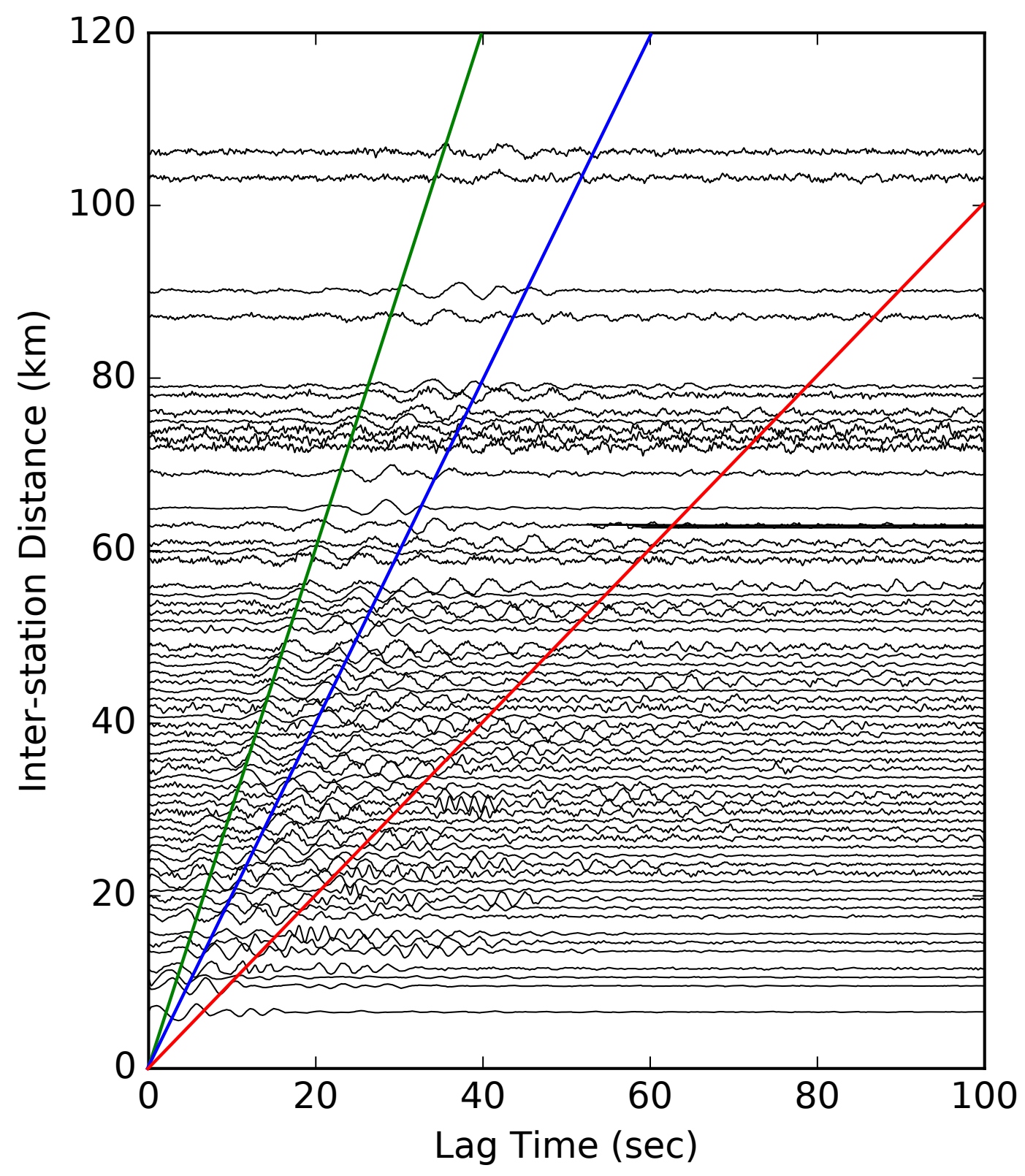

Figure 4.2: Vertical component cross-correlations as a function of inter-station distance. Broadband ZZ cross-correlation functions for all stations pairs are shown for positive lag times, to $100 \mathrm{~s}$. Lines with moveout velocities of $1 \mathrm{~km} . \mathrm{s}^{-1}$ (red line), 2 $\mathrm{km} . \mathrm{s}^{-1}$ (blue) and $3 \mathrm{~km} . \mathrm{s}^{-1}$ (green) are shown as a guide to moveouts present across the study region. 
seismograms from one station were cross-correlated with the other's to yield nine cross-correlation functions (ZZ, ZR, ZT, RR, RT, RZ, TT, TR, TZ), approximating the nine-component Green's function tensor. The two signals were cross-correlated in fifteen-minute windows and stacked to produce daily cross-correlation functions (Fig. 4.1, bottom panel). For the resulting signals with positive time lags, the first letter represents the response of a force in that polarisation at the first station, to be recorded by the corresponding component of the second station, denoted by the second letter. This is reversed for negative time lags (Shapiro and Campillo, 2004). The daily functions are stacked for each station pair over the whole study period, of 4 months, to produce reference stacks in the 0.1 to $1.0 \mathrm{~Hz}$ frequency band. For every consecutive 10 days, shorter stacks were calculated for the same frequency band, herein referred to as moving stacks. Figure 4.2 plots the cross-correlations of the ZZ components for the entire dataset as a function of time and inter-station distance.

The MWCS method (Clarke et al., 2011) was used to examine velocity variations. The short duration 10-day stacks of cross-correlations were compared to the reference stacks. The choice of moving stack length is determined by considering the length of the reference stack and the time scale on which velocity changes are expected to occur. The moving stack should be sufficiently short in comparison to the reference stack so that changes can be detected as we are considering the long-term recovery of the region. Taking this into account and to ensure that the moving stacks are stable through time, a 10-day stack was considered appropriate. A value of coherence and time delay between the reference stack and the moving stacks for each 10 second segment was computed (Figure 4.3 inset). For coherence values below 0.7 the time delay measurement was discarded from subsequent processing. The slope of the time delays versus lag time for each moving stack gives an overall percentage time delay from the reference stack for each moving stack (Figure 4.3). The inverse of the slope of the delay time versus lag time gives a 10-day value of relative velocity change, which is the percentage velocity deviation from the reference stack (Clarke et al., 2011). The MWCS method is performed on each of the nine cross-correlation components for each station pair. For each component, results from all station pairs on a given day are combined to give a network-wide average weighted by delay time uncertainty. The two temporary deployments (both augmented by permanent stations) were processed separately as there was only a 10-day overlap when both stations were recording. The broadband deployment was broken into two sets of measurements because there was a six week gap between the end of November and mid-December when data from several stations were missing or unsuitable for processing. As the MWCS measurements only give relative velocity measurements, 
we needed to correct for the likely absolute differences in velocities for the three sets of deployments. For the short period and broadband deployments there was an overlap of ten days, so we assumed that the average velocities within the two sets of stations were the same, and corrected the broadband station's velocity changes by the average difference between the two sets of velocities over the common deployment time. Correcting for the last set of data was more difficult because there was no closely-spaced network operating with which to compare. Therefore we made an assumption that processes controlling the increase in velocity during the first two deployments continued at the same rate. We then fit the increase in velocity by a curve of the form $y=\operatorname{alog}(x / b)$ and extrapolated the curve to the time of the last set of velocity changes, fixing the zero for the last set to the extrapolated velocity change. We also extrapolate the curve backwards in time to determine an estimate of the possible total velocity change since the earthquake. These last extrapolation steps are considered most tenuous, so we do not interpret it except to compare the consistency of the assumption with the measured velocity changes to other studies.

Several quality control measures were implemented through the processing procedure. Only station pairs with a minimum inter-station distance of three wavelengths were considered, so that several full surface wave cycles were completed between stations (Lin et al., 2007). In addition, when computing MWCS delay times, to examine only the surface wave, cross-correlation lag times that give surface wave velocities outside the range $0.7-4.0 \mathrm{~km} . \mathrm{s}^{-1}$ are discarded, dependent on inter-station distance and assuming a simple speed $=$ distance $\mathrm{x}$ time relationship.

\subsection{Results}

Figure 4.1, bottom panel, shows an example daily cross-correlation function for the on-fault station pair DAR04-DAR07 compared to its corresponding reference function for the two months with the longest continuous duration. Incoherent noise signals at longer lag times have been suppressed in the stacked trace and the surface wave arrivals are emergent and less contaminated with random noise. For this representative station pair (Figure 4.1, bottom panel), signal amplitudes on the negative Green's function, corresponding to waves travelling from east to west, are systematically larger than the positive response. The inter-station path is orthogonal to the coastline, and so ocean waves travelling in from the coast towards the west dominate the noise field (Brooks et al., 2009; Behr et al., 2013). Cross-correlations as a function of interstation distance confirm average group velocities of $1-3 \mathrm{~km} / \mathrm{s}$ (Figure 4.2), consistent with earlier studies covering the region using subsets of this 
data (Savage et al., 2013; Fry et al., 2014).

Relative velocity variations are shown in Figure 4.4 for 0.1 to $1 \mathrm{~Hz}$ frequencies. The variations have been plotted relative to the beginning of the study period in midSeptember 2010. The output of MWCS gives velocity differences from the average reference cross-correlation of all components for each station pair, shown in Figure 4.4 as the dashed black and solid blue lines.

The velocity increases for each component are plotted separately (thin grey lines). There is an overall increase of $0.13 \pm 0.04 \%$ and variations between components can be seen (Figure 4.4). As explained in the previous methods section, in order to examine the overall regional velocity change following the earthquake, the results for the second dataset are corrected by a constant to follow the trend of the GeoNet rapid response results, giving a pseudo-continuous velocity change curve. Although the stations sample the same overall region, the two temporary networks do not have the same lateral sensitivity. The correction assumes that the crustal seismic velocity recovery is laterally homogeneous over the entire region as sampled by the seismic networks. Spatial changes in velocity variations across the region are not strongly apparent. Figure 4.5 shows velocity change curves for interstation paths including OXZ and DAR06. Station OXZ lies in the northwest corner of the study area, approximately $30 \mathrm{~km}$ to the north of the fault trace. DAR06 is situated in the centre of the fault trace. The stations do not have obvious differences in their trend, despite the differing locations. Using a longer stack does not provide enough measurements to confidently examine spatial variations. Once average velocity increases for each component (Figure 4.4) are calculated, clearer trends are visible. This is due to each $d t / t$ measurement being weighted according to time delay coherence and measurement uncertainty.

\subsection{Discussion}

The surface wave velocity change of $0.13 \pm 0.04 \%$ over the two and a half month study period is comparable to that observed in other similar studies elsewhere. A $0.02 \%$ increase in velocity was recorded in the two months immediately following the lower magnitude $\mathrm{M}_{w}$ 6.0 Parkfield earthquake in California (Brenguier et al., 2008). Pre-seismic levels were measured using continuous seismic waveforms from several years prior to the earthquake and velocities returned to pre-seismic levels at the same rate as GPS displacements over several years (Brenguier et al., 2008). Using auto-correlations of seismic waveforms following the 2011 Tohoku-Oki $\mathrm{M}_{w}$ 9.0 event, Minato et al. (2012) observed a $1.5 \%$ velocity increase for the same 


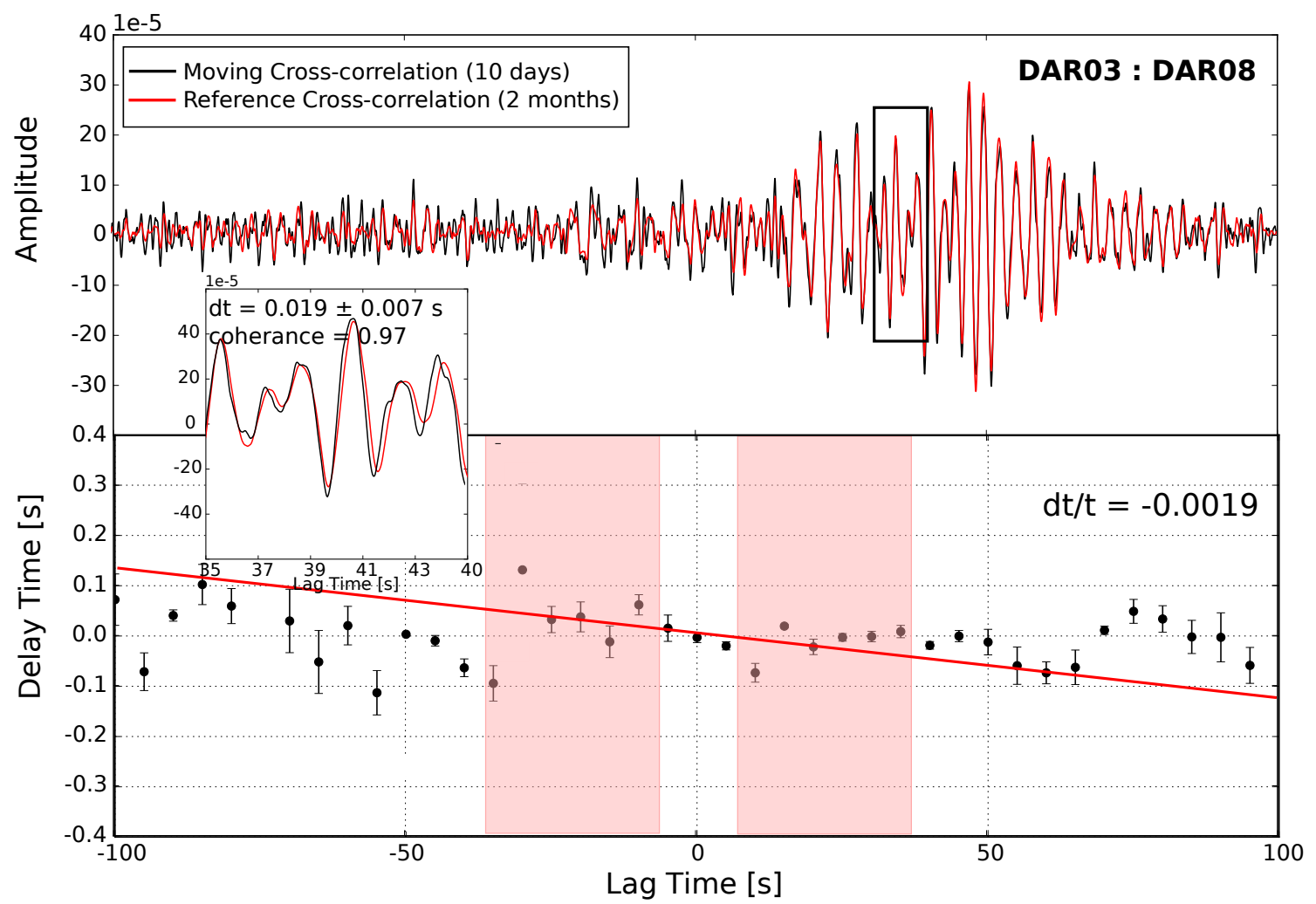

Figure 4.3: Example of delay time versus lag time using the MWCS method for a representative 10-day stack for station pair DAR03-DAR08. Top panel shows the reference stack (red) and a representative 10-day stack for the 10 days prior to and including 21 November 2010 (black line). The inset shows an enlarged section of the cross-correlations highlighted by the black box. For the 10 second period, the delay time and corresponding coherence are calculated. This result contributes a point on the delay time versus lag time (lower panel) for the same day. The slope of a linear regression for lag times within the pink boxes, which are selected according to interstation distances, gives the relative delay time for that day. 


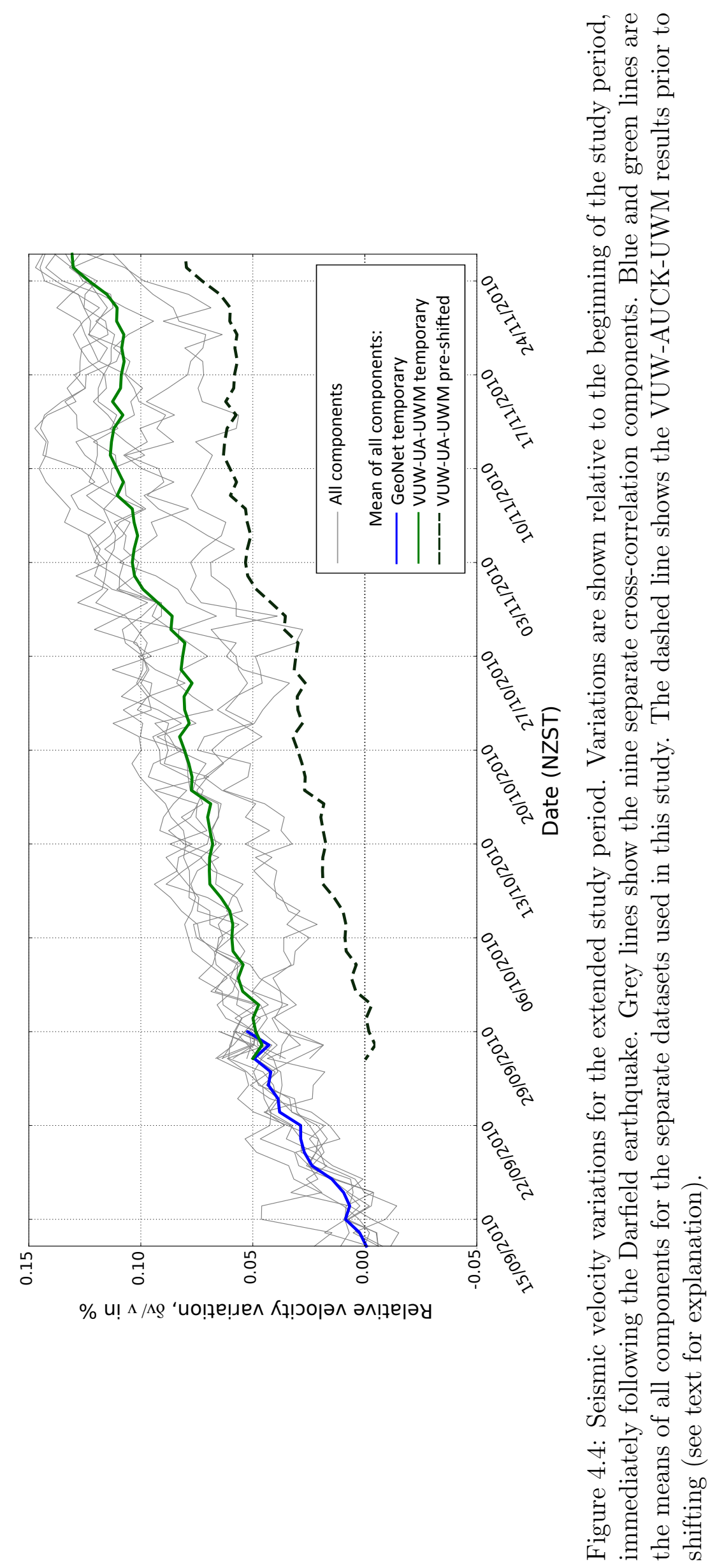


Table 4.1: Relative velocity increases by cross-correlation component. All measurements have $95 \%$ confidence intervals of $\pm 0.01 \%$.

\begin{tabular}{c|c}
\hline Component & Velocity Increase (\%) \\
\hline ZZ & 0.13 \\
RR & 0.15 \\
TT & 0.13 \\
ZR & 0.15 \\
RZ & 0.14 \\
ZT & 0.12 \\
RT & 0.14 \\
TR & 0.12 \\
TZ & 0.14 \\
\hline
\end{tabular}

two month time period. In this study, it was assumed that the seismic velocities throughout the region were increasing steadily following an initial rapid co-seismic decrease from average seismic velocities, as has been observed in other locations (e.g. Brenguier et al., 2008; Minato et al., 2012). Pre- and co-seismic velocity levels cannot be determined in this study as the majority of the data are from rapidresponse networks. Analysis was performed on several of the permanent continuous national stations in an attempt to seek a pre-seismic signal, but it was found that these stations were too distant from the Greendale Fault to achieve a high enough signal-to-noise ratio, to record any short-term velocity changes. Thus we do not have enough data to determine pre- versus post-earthquake changes.

If the Green's function tensor components are examined separately (Figure 4.6, Table 4.1), cross-correlations with a transverse component (i.e. TT, ZT, TR) show slightly smaller increases in velocity (up to $0.03 \%$ ) compared to those without (i.e. RR, ZZ, RZ, ZR), which predominately record Rayleigh waves. This could suggest that Rayleigh waves are perturbed by subsurface structures more than Love waves. However, these variations are all within the bounds of uncertainty for the delay time measurements (Table 4.1). There are some small, erratic, short term velocity changes seen on all components that are not accounted for by measurement error and do not correlate with time across components. They are likely errors introduced by the recorded raw data, either by missing data or recording glitches that were not sufficiently suppressed during pre-processing.

Figure 4.7 shows the modelled fit to the data, with $95 \%$ confidence intervals. The data used for modelling were the GeoNet temporary network results and the VUWUA-UWM results until the end of November 2010 (red closed and open circles). Results for the end of December 2010 and beginning of January 2011, follow a 6-week gap, where data from several stations were missing or unsuitable for processing. The 


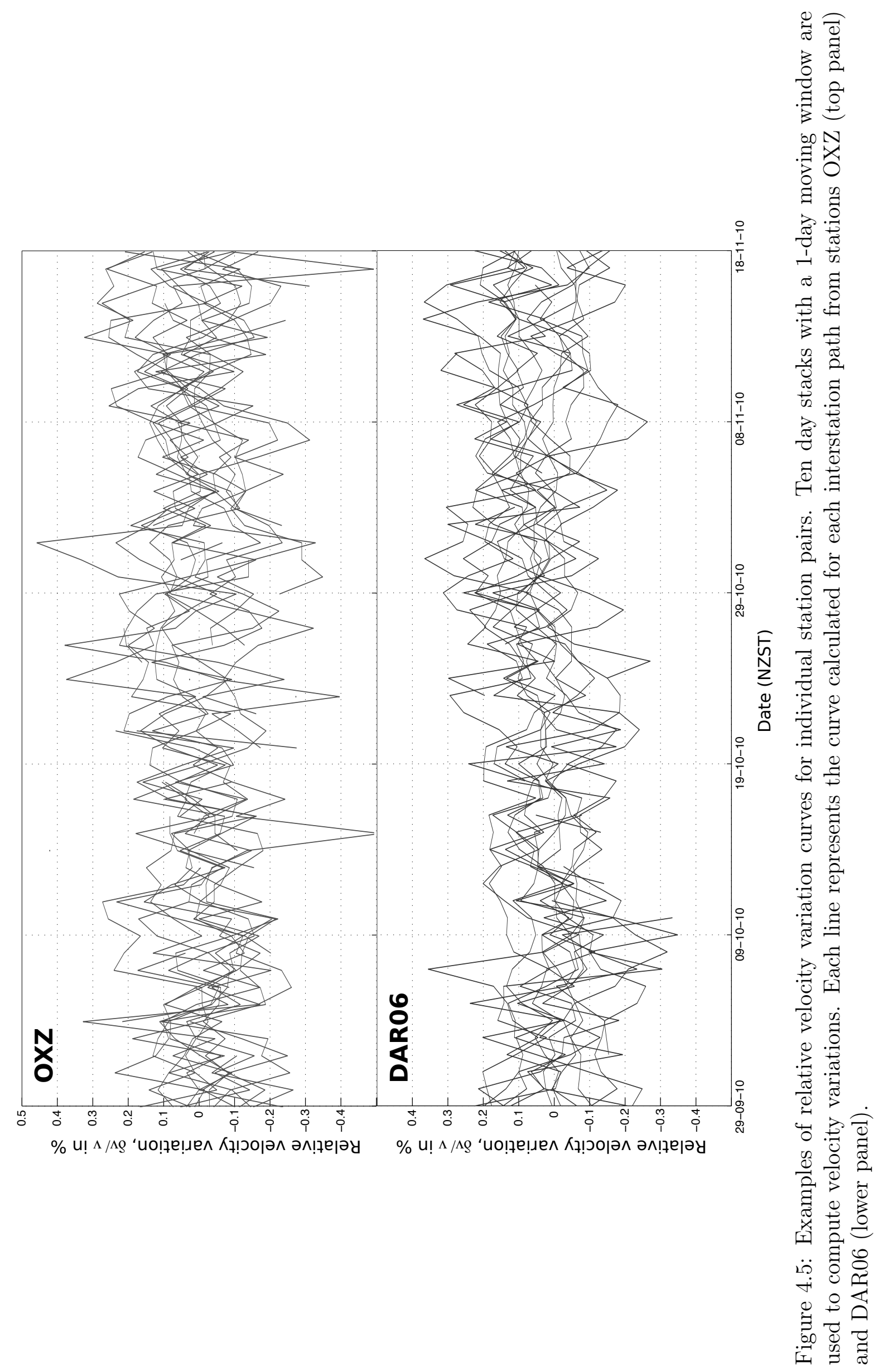




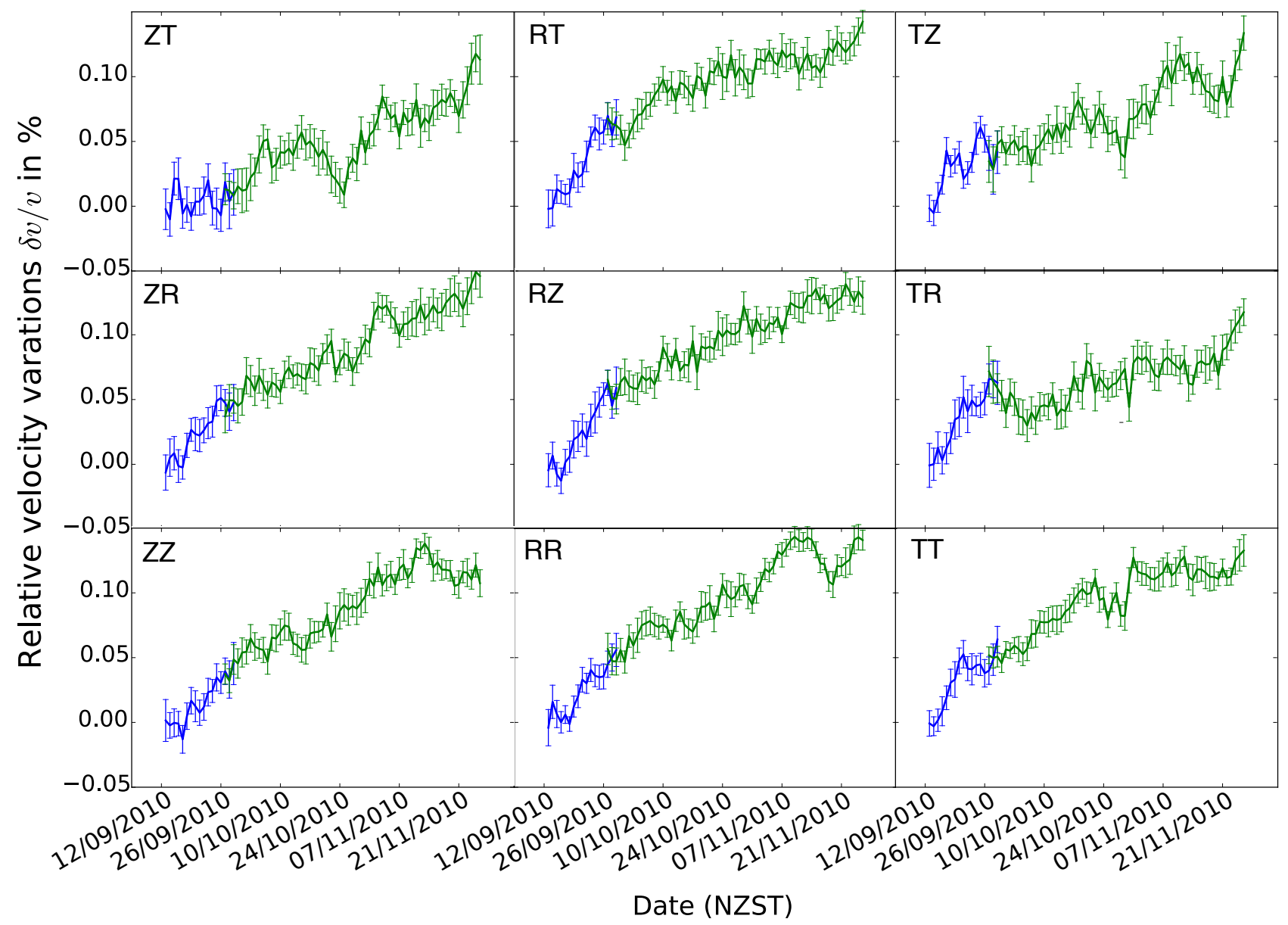

Figure 4.6: Seismic velocity variations for the nine cross-correlation components for the GeoNet (blue) and VUW-UA-UWM (green) datasets with associated 95\% confidence intervals. 
results for this later time period were processed separately from the other datasets (Fig. 4.7, closed blue circles), and then shifted to fit the model, according to the average difference between the data points and the curve, as discussed in the methods section. The total seismic velocity increase for the 140 days following the earthquake is estimated to be $0.14 \%$. The seismic velocity increase over the period for which we have most control is $0.1 \pm 0.01 \%$ for the times between 10 and 85 days after the earthquake. The slope of the velocity changes between 110 and 130 days are consistent with extrapolation of the curve between 10 and 85 days, which would suggest a $0.14 \%$ change between 10 and 130 days after the earthquake.

To assess possible mechanisms for the postseismic velocity changes, the results for vertical-component cross-correlations have been divided into several narrow frequency bands (Figure 4.8, left panel). Velocities increase steadily for frequencies higher than $0.2 \mathrm{~Hz}$, with the largest increases of up to $0.25 \%$ occurring within $0.5-$ $1 \mathrm{~Hz}$, with velocities increasing over the whole time period considered. The $0.2-0.5$ $\mathrm{Hz}$ results show a steady increase to $0.12 \%$ until the end of October, then seismic velocities stabilise at these frequencies. Lower frequencies of $0.1-0.2 \mathrm{~Hz}$ do not show any increase in velocity over the study period. Figure 4.8 (right panel) gives fundamental mode Rayleigh wave sensitivity kernels for frequencies considered in this study, generated using an average regional velocity model (?) and the codes of Herrmann and Ammon (2004). At shallow depths the depth of maximum sensitivity in kilometres is approximately equal to the inverse of the surface wave frequency. Therefore the highest velocity increases of $0.25 \%$ are occurring in the uppermost 2 $\mathrm{km}$. The waves in the analysed frequency range have little sensitivity below $5 \mathrm{~km}$ depth. Comparable results have been seen for other large earthquakes. The 2003 San Simeon and 2004 Parkfield earthquakes in California showed that seismic velocity increases were largest for short periods less than $1 \mathrm{~s}$, or $1 \mathrm{~Hz}$ frequency, up to 0.2 $\%$, with periods greater than $1.6 \mathrm{~s}(0.625 \mathrm{~Hz})$ showing very little long term increase (Wu et al., 2016). Liu et al. (2014) and Hobiger et al. (2012) demonstrated similar results with negligible velocity increases at long periods for the 2008 Wenchuan and 2008 Iwate-Miyagi Nairiku events respectively.

Several possible mechanisms for velocity changes following earthquakes have been proposed, as summarised by Xu and Song (2009). Firstly, seismic velocities could be affected by short-term groundwater responses and other fluid movements. Cox et al. (2012) and Gulley et al. (2013) observed groundwater responses following the Darfield earthquake throughout the region, on timescales of hours to days. Here, a moving window length of 10 days was considered, so any short-term changes would not be recorded. A second possible mechanism is through damage of shallow crust from strong ground shaking. This is one likely mechanism present here, as the 


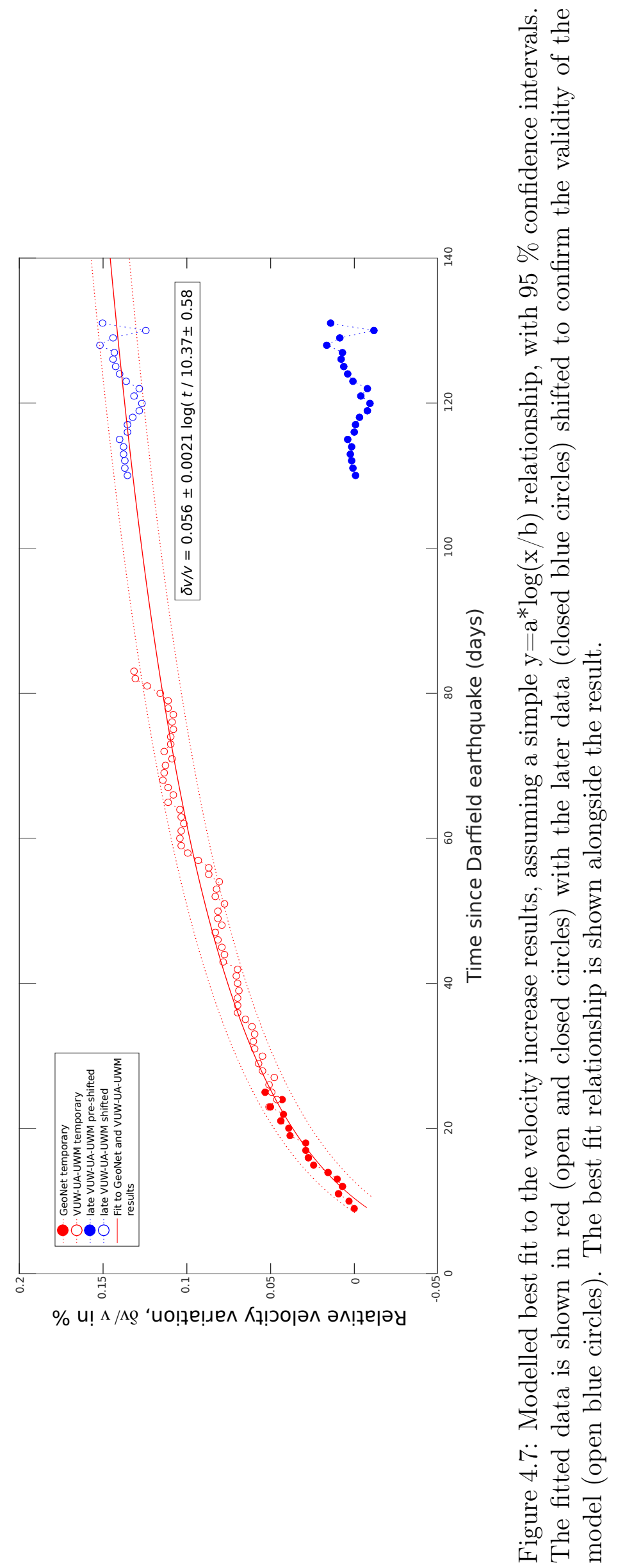




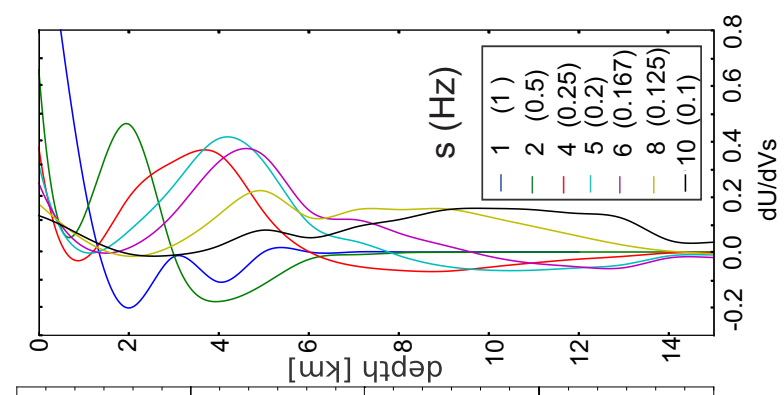

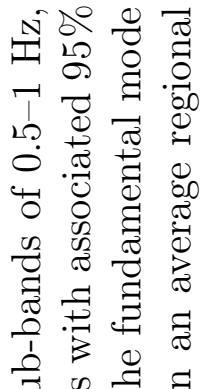

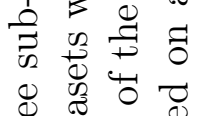

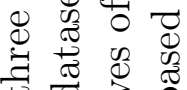

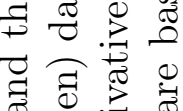

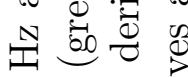

十之空

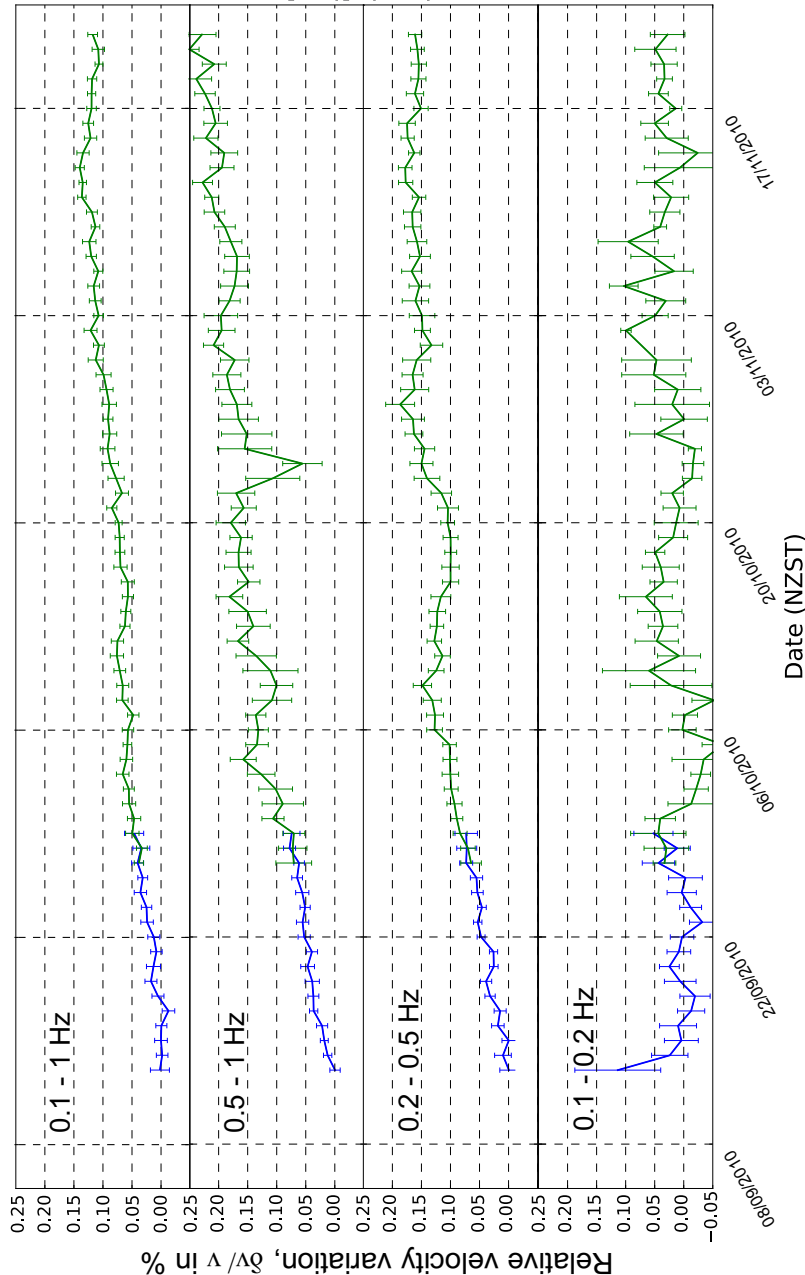

它宫 总

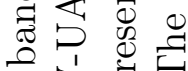

$B D E$

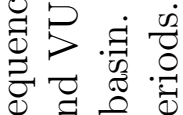

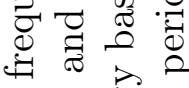

일

$\exists \overline{\mathrm{e}}$

青艺芯

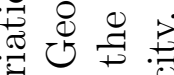

ॠँ

ปั

령ㅎㅇ

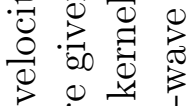

○.

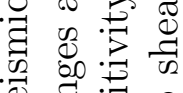

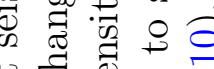

킹 छे

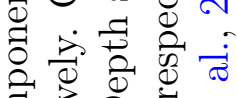

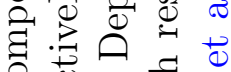

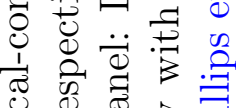

.

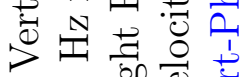

뭉.

藏

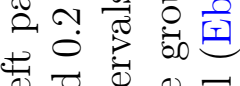

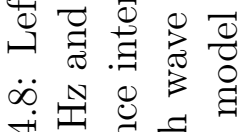

○

品 
frequencies of 0.1 to $1 \mathrm{~Hz}$ analysed equate to relatively shallow crustal depths of approximately $1-10 \mathrm{~km}$. One contributing mechanism likely is damage from the fault-zone rupture and subsequent healing of cracks. Velocity changes are seen across the region, not just for station pairs with paths crossing the Greendale Fault, indicating that the healing of micro-cracks in addition to crack-healing of the Greendale Fault, was the dominant factor for change. The lack of difference between horizontal and vertical components suggests that there is an isotropic stress state throughout the region following the earthquake.

This work has successfully shown an increase in surface wave velocities throughout the Canterbury region following the Darfield earthquake using the moving-window cross-spectral method. Average changes of up to $0.15 \%$ were seen down to 10 $\mathrm{km}$ depth across all 9 components of the Green's function tensor, with the largest velocity increases occurring in the uppermost $2 \mathrm{~km}$. This method complements other studies following large earthquakes to examine the post-seismic stress relaxation and recovery of fault zones.

\subsection{Acknowledgements}

This work was funded by grant VUW1312 from the NZ Marsden fund and a Victoria University scholarship. The broadband temporary station data were collected using funding from NSF grant EAR-1102767 and grant 10/CE618 from the New Zealand Earthquake Commission, and are available from the IRIS data management centre. Instrumentation for the temporary deployments was provided by PASSCAL, GNS Science Wairakei and the University of Auckland. GeoNet provided permanent and temporary seismic station data. Cross-correlations and velocity variations were computed using the MSNoise processing package. Comments on earlier versions of the manuscript were provided by F. Brenguier, A. Ferreira and two anonymous reviewers. 


\section{Surface and Shear Wave Velocity Models of the Southern Alps, New Zealand}

\subsection{Introduction}

The Alpine Fault runs northeast-southwest, with an average strike of $055^{\circ}$, down the west coast of the South Island, New Zealand. It is a mature dextral transform fault with a surface trace of over $800 \mathrm{~km}$ (Sutherland et al., 2007). The fault represents the continental part of the boundary between the Pacific and Australian continental plates, with subduction of opposite polarities to the north and south (Walcott, 1998; Okaya et al., 2007). To the north of South Island is the Hikurangi subduction zone dipping to the West, changing to the east-dipping Puysegar subduction zone further South. The Alpine Fault marks the transition between these two regions, accomodating up to $75 \%$ of the strike-slip component of the relative plate motion and almost all of the dip-slip component (Norris and Cooper, 2001).

There are no historical records of large earthquakes occurring on the fault since c. $1800 \mathrm{AD}$, however paleoseismicity has shown an average recurrence interval of 350 years for events $\mathrm{M}>8.0$ (Berryman et al., 2012). Tree ring data suggested the surface ruptures of the most recent events in 1430, 1620 and 1717 AD were several hundred kilometres, each with magnitudes greater than 7.5 (Sutherland et al., 2007, Figure 5.1). Norris and Cooper (2001) observed a current slip rate of $2-3$ cm. $\mathrm{yr}^{-1}$.

As the Alpine Fault is considered to be late in its earthquake cycle (Sutherland et al., 2009), and poses one of the largest seismic hazard currently for New Zealand, interest in the fault and surrounding region has greatly increased in recent years. Several large scale projects have sought to understand and model the crustal structure and fault properties. The South Island Geophysical Transect (SIGHT) project used active source methods to image the seismic velocity structure (Okaya et al., 2007; Stern et al., 2007). Using seismic reflection methods for two transects running onand off-shore perpendicular to the fault, P-wave velocity models were produced 


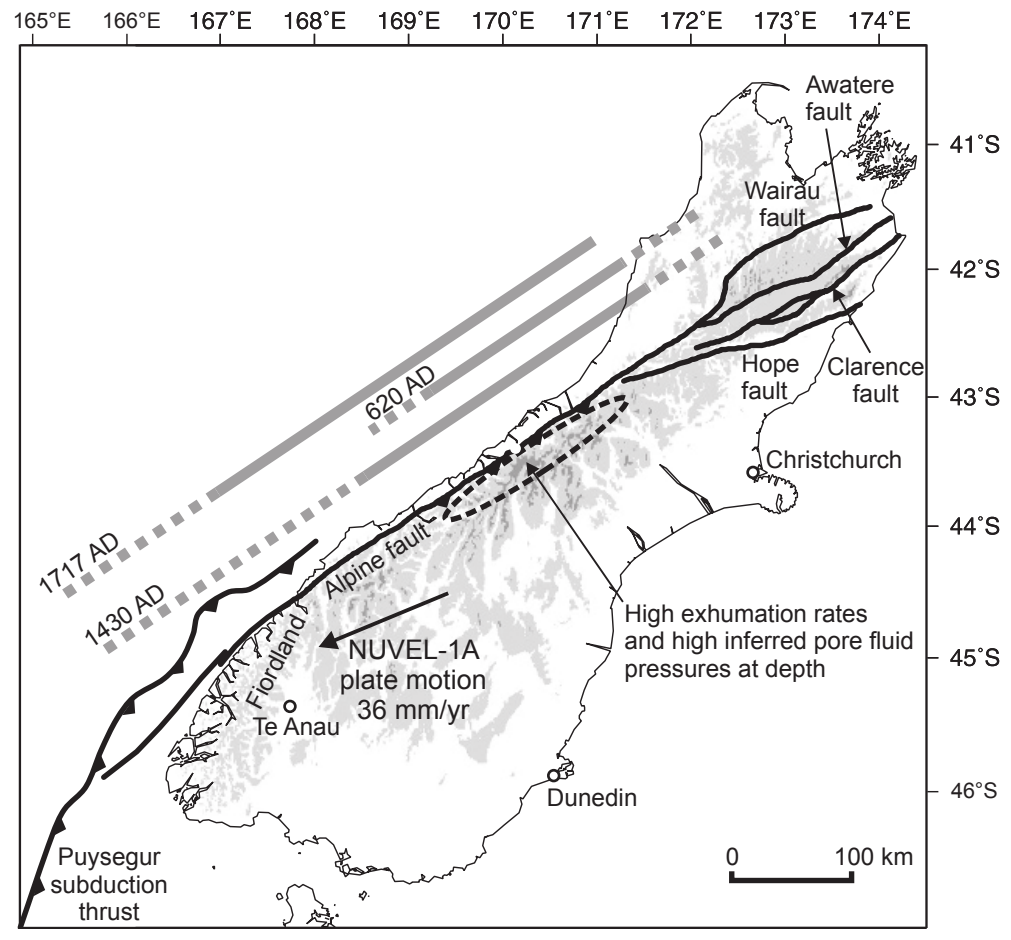

Figure 5.1: Location of the Alpine Fault in South Island, New Zealand. The surface rupture extents of inferred historic large earthquakes are highlighted. After Sutherland et al. (2007).

(Stern et al., 2007; Van Avendonk et al., 2004). The Alpine Fault was traced at depth, with an apparently listric structure. Stern et al. (2007) found a zone of reduced velocities in the hangingwall of the fault, which coincided with an area of low resistivity. These results were in agreement with those of the Southern Alps Passive Seismic Experiment, or SAPSE (Eberhart-Phillips and Bannister, 2002). This study inverted $\mathrm{P}$ and $\mathrm{P}-\mathrm{S}$ arrival times from earthquake and active shot data to obtain $\mathrm{V}_{P}$ and $\mathrm{V}_{P} / \mathrm{V}_{S}$ ratio models. $\mathrm{P}-$ wave velocities obtained for $5-25 \mathrm{~km}$ depth were found to be fairly uniform and typical of greywacke and schists. The Alpine Fault was characterised by a low-velocity zone to at least $15 \mathrm{~km}$ depth (Eberhart-Phillips and Bannister, 2002).

Lin et al. (2007) shows at long periods of 18 and $23 \mathrm{~s}$, a well defined region of high Rayleigh-wave group velocities, ranging between $3.0-3.5 \mathrm{~km} . \mathrm{s}^{-1}$. This was interpreted as the Southern Alps and Fiordland. However to date the ambient seismic noise field of the Southern Alps has not been investigated on a small-scale, and an accurate shallow subsurface S-wave velocity model is lacking for the central Alpine Fault region. 


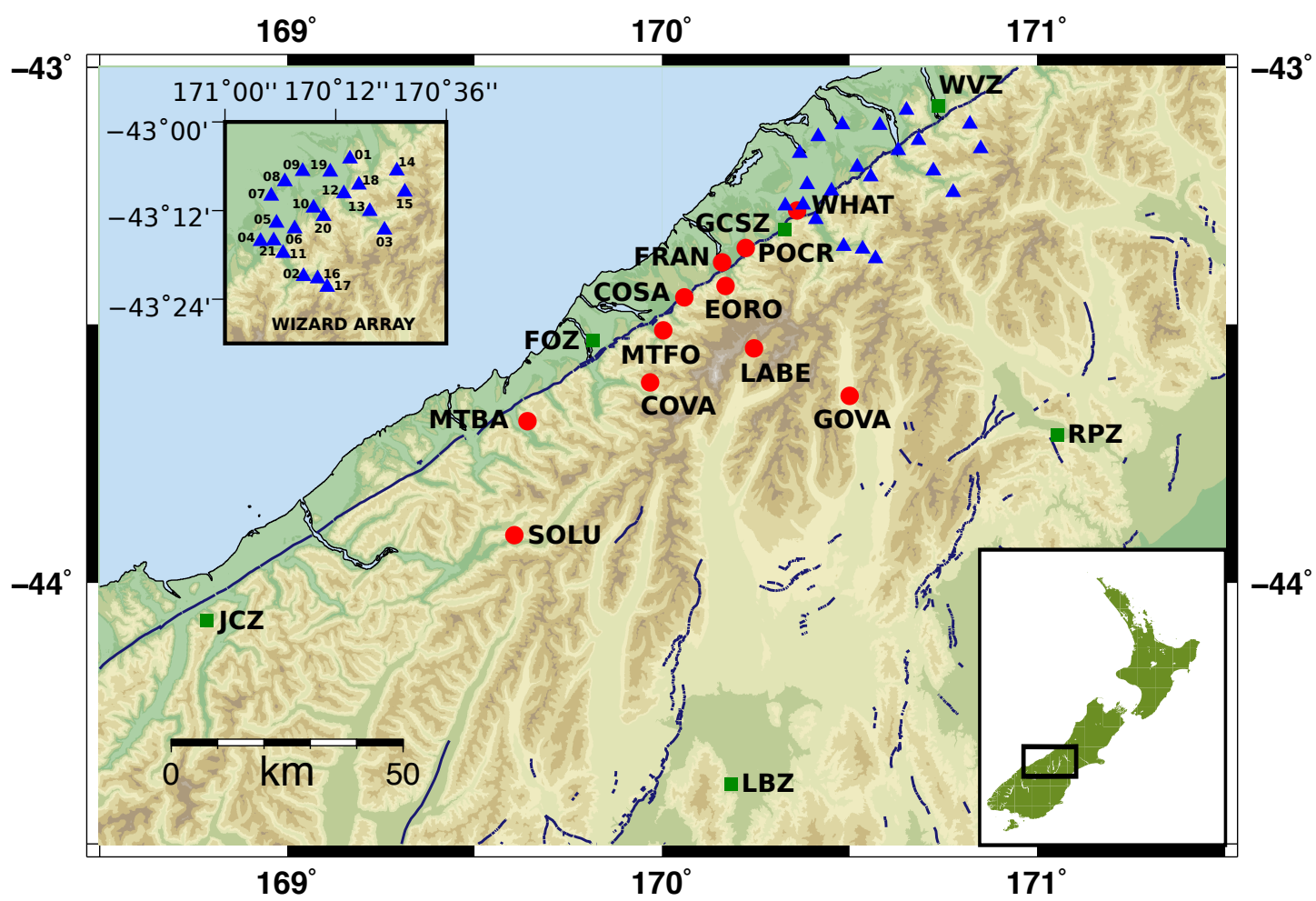

Figure 5.2: Map of the central Southern Alps region. Mapped faults are shown by thin dark grey lines. Coloured symbols show locations of GeoNet permanent broadband stations (green squares), SAMBA network stations (red circles), and the WIZARD array (blue triangles). Insets show the numbering of the WIZARD array stations and the location of the study area within New Zealand (top left and lower right respectively.

\subsection{Data}

The central locked region of the Alpine Fault has a wealth of seismic data available. The data used in this study are continuous waveform data from 2013 recorded on several seismic networks (Figure 5.2).

The SAMBA (Southern Alps Microseismic Borehole Array) network (Boese et al., 2012) was first deployed in 2008 to record microseismicity in the region and has since been used to identify low frequency earthquakes (Chamberlain et al., 2014, 2017). The network consists of ten borehole and four surface sensors. The stations are mostly concentrated along the surface expression of the central Alpine Fault, in addition to several seismic stations located in the Alpine mountain ranges (Figure 5.2 , red circles).

Thurber et al. (2012) deployed the WIsconsin New Zealand And Renssalaer Deployment, or WIZARD, array in 2012, which was operational for two years. Twenty-one broadband and short-periods stations recording in 2013 were used in this study. 
The WIZARD network is concentrated around the site of the DFDP-1 (Deep Fault Drilling Project) borehole (Sutherland et al., 2009) and was deployed with the aims of recording regional seismicity, microseismicity and undertaking earthquake tomography (Thurber et al., 2012).

The nearby regional stations of the national permanent GeoNet network (Petersen et al., 2011), FOZ, GCSZ, LBZ, RPZ and WVZ supplemented the other networks. All GeoNet stations are broadband, except the short-period borehole station GCSZ. The availability of data for all stations throughout 2013 is shown in Figure 5.3. Further information about each deployment is presented in Appendix A.

\subsubsection{Data Preparation}

The quality of the raw data was examined prior to processing. As in section 3.2.1, days that were not $86400 \mathrm{~s}$ in length were removed from the data pool. On receiving the SAMBA data it was discovered that gaps had already been padded with zeros, so it was necessary to remove any such sections. This reduced the usable data somewhat, but as shown in Figure 5.3 there was still a sufficient volume of data to continue with processing. Some stations had issues with systematic electrical spikes, such as POCR. These were removed through filtering and the rglitch function of the SAC codes (Goldstein and Snoke, 2005).

\subsubsection{Rotation of Raw Horizontal Components}

All of the horizontally recorded SAMBA borehole stations had to be rotated to true north and east, as the sensors rotate when lowered into the borehole during installation. Sensor orientations at depth can be estimated using particle motions of direct body waves recorded for teleseismic, regional and local earthquakes (Aster and Shearer, 1991; Chiu et al., 1994). For the SAMBA array, the determined orientations are given with uncertainties in Appendix A (Boese, C. and Zal, H., pers. comm).

Instrument response files were produced from the metadata, or dataless SEED files for both the SAMBA and WIZARD networks, using the IRIS EVALRESP package. The amplitude and phase responses for the instruments considered here are shown in Figure 5.4. The main comparison that can be drawn from the curves is the differing corner frequencies between the broadband (Guralp CMG3-ESP) and short period (Marks L22, Marks L4, Duke GC2) sensors of $0.02 \mathrm{~Hz}$ and $2 \mathrm{~Hz}$.

The mean and trend were removed from each daily trace and a cosine taper of 


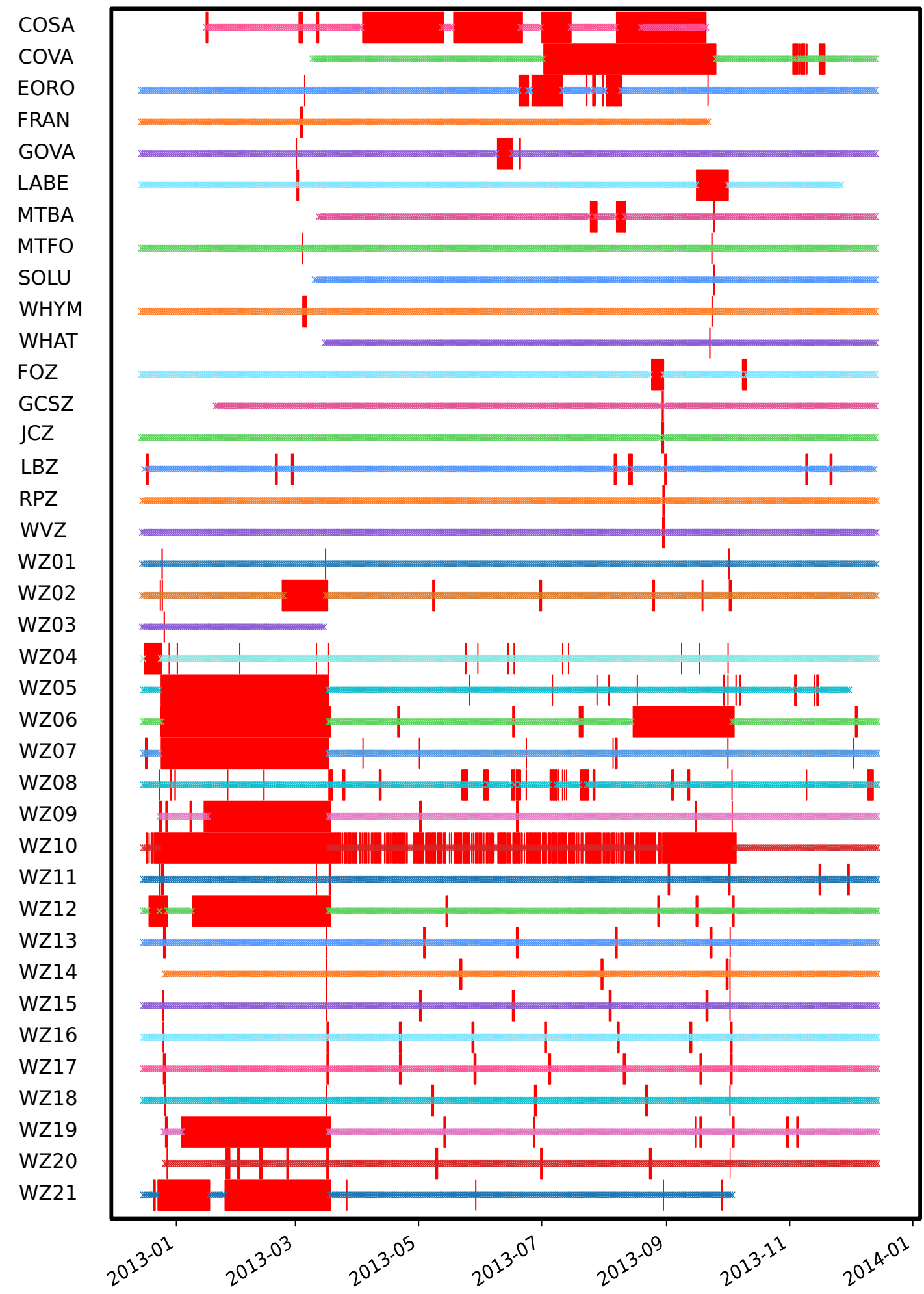

Figure 5.3: Schematic diagram of data availability for the all seismic stations used in this study. Crossed indicate present data for a given day and red blocks show gaps in the dataset. More detailed station information can be found in Appendix A. 
$1 \%$ is applied to each end of the waveform. The instrument response was then removed. As this study uses networks with broadband, short-period, surface and borehole instruments, removal of the phase and amplitude responses is extremely important and the resulting waveforms were heavily scrutinised. The same procedure as in section 3.2.1 is used, by selecting a teleseismic earthquake and examining the effects. In our frequency band of interest, $0.1-2 \mathrm{~Hz}$, we would expect that the amplitudes of signals will not change significantly for broadband stations, but there will be some amplitude increase in the lower frequencies of the short period sensors. As one-bit normalisation is implemented, the phase correction of the instrument response removal is the more important than amplitude corrections.

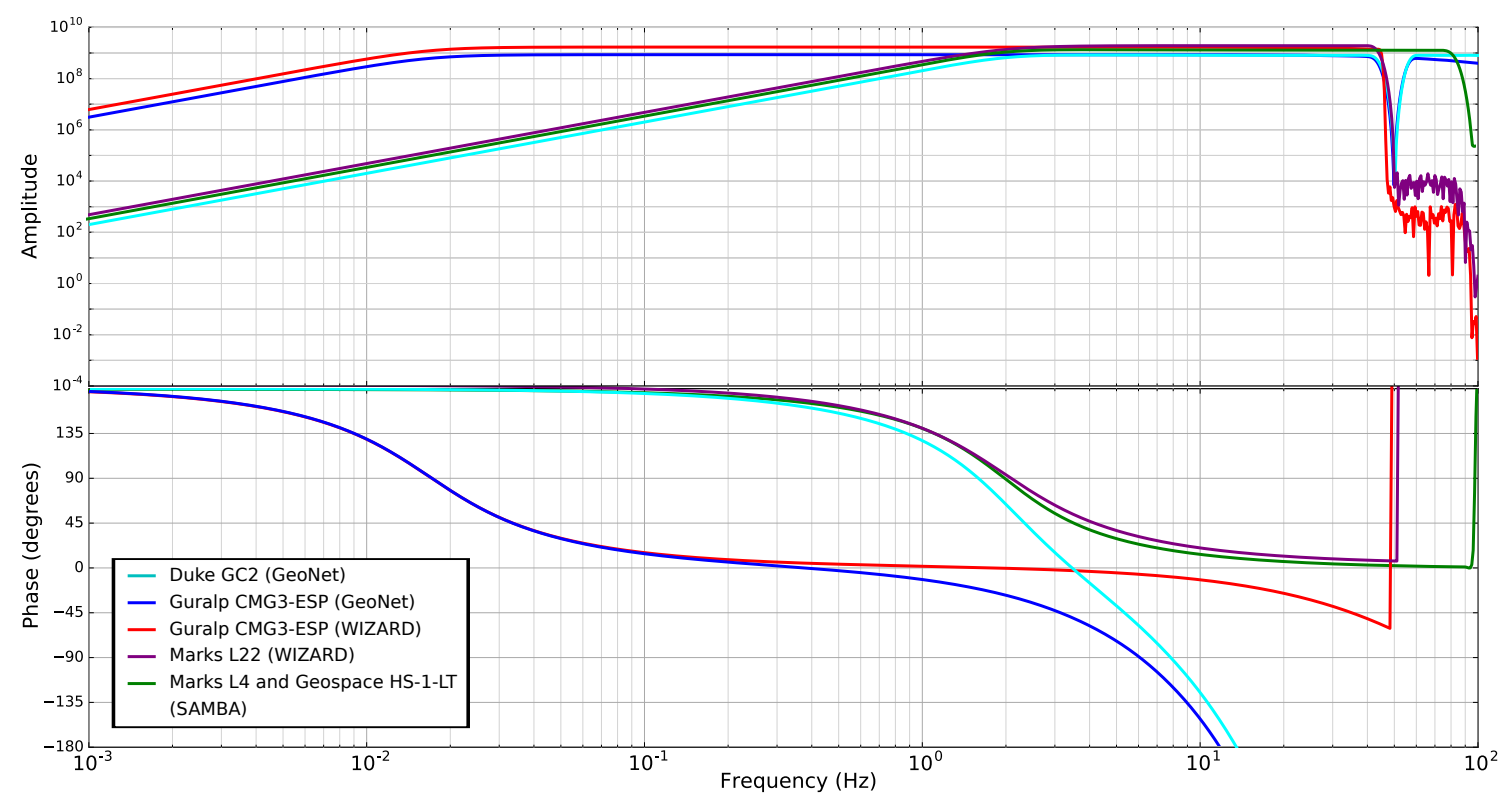

Figure 5.4: Amplitude and phase response curves for the sensors used in this study. GeoNet station GCSZ uses a short period Duke GC2 sensor, all other GeoNet stations use broadband Guralp CMG3-ESP instruments. SAMBA stations are either Marks L4 or Geospace short period sensors (further information given in Appendix A) and the WIZARD deployment comprised Marks L22 and Guralp broadband sensors.

\subsection{Methods}

The processing methods used for this study location largely follows the same scheme as Chapter 3. 
Table 5.1: Cross-correlation processing parameters used for the Alpine Fault study

\begin{tabular}{|l|l|}
\hline Sampling frequency & $25 \mathrm{~Hz}$ \\
\hline Pre-processing bandpass filter & $0.01-12 \mathrm{~Hz}$ \\
\hline Time-domain normalisation & one-bit applied \\
\hline Whitening filter & $0.05-2 \mathrm{~Hz}$ \\
\hline Cross-correlation segment length & 15 minutes \\
\hline Maximum correlation time lag & 120 seconds \\
\hline SNR threshold & 5 \\
\hline
\end{tabular}

\subsubsection{Cross-correlation Computation}

Table 5.1 summarises the parameters used for obtaining the cross-correlations. Stacked cross-correlation functions were computed using the methods explained in Section 2.3 and the MSNoise processing package (Lecocq et al., 2014). Data were resampled to $25 \mathrm{~Hz}$ and bandpass filtered between 0.01 and $12 \mathrm{~Hz}$. Spectral whitening between 0.1 and $2 \mathrm{~Hz}$ and one-bit normalisation were applied to all daily records. Radial and transverse traces were obtained by rotating the correctly oriented horizontal waveforms. The cross-correlation components ZZ, RR and TT were computed by cross-correlating each station pair in 15 minute windows. These are stacked to produce daily functions and monthly stacks. A single cross-correlation function is also produced for each component of each station pair by stacking over all available days. The lengths of the final stacked functions range from 147 days to 365 days. After applying several quality controls which are described in the following section, dispersion curves are obtained from the correlations.

Figure 5.5 shows cross-correlation functions for the ZZ, RR and TT components for station pair LBZ-WZ18. The surface wave arrives approximately $10 \mathrm{~s}$ earlier for TT than the other components. Transverse Green's functions contain Love wave arrivals, so this earlier arrival is as expected, as Love waves typically travel up to $10 \%$ faster than Rayleigh waves (Stein and Wysession, 2003). LBZ-WZ18 has an interstation path of $143.9 \mathrm{~km}$. The Rayleigh wave arrives at a lag time of approximately $48 \mathrm{~s}$ and the Love at $42 \mathrm{~s}$. This gives approximate speeds of $3.0 \mathrm{~km} . \mathrm{s}^{-1}$ and $3.4 \mathrm{~km} . \mathrm{s}^{-1}$. Although crude, simple checks of wavespeeds in the cross-correlation functions can assist in identifying erroneous functions and unexpected velocities in inversions of dispersion curves and tomography. The presence of anisotropy can be assessed in the cross-correlation functions by examining all nine components. In figure 5.6, the positive-lag cross-correlations are shown for station pair LBZ-WZ18. The vertical and radial components (ZZ, RR, RZ, ZR) all show the Rayleigh wave arrival at approximately $48 \mathrm{~s}$, and the amplitudes are consistent across all components. If higher modes we're present, it would be expected that the first arrivals 


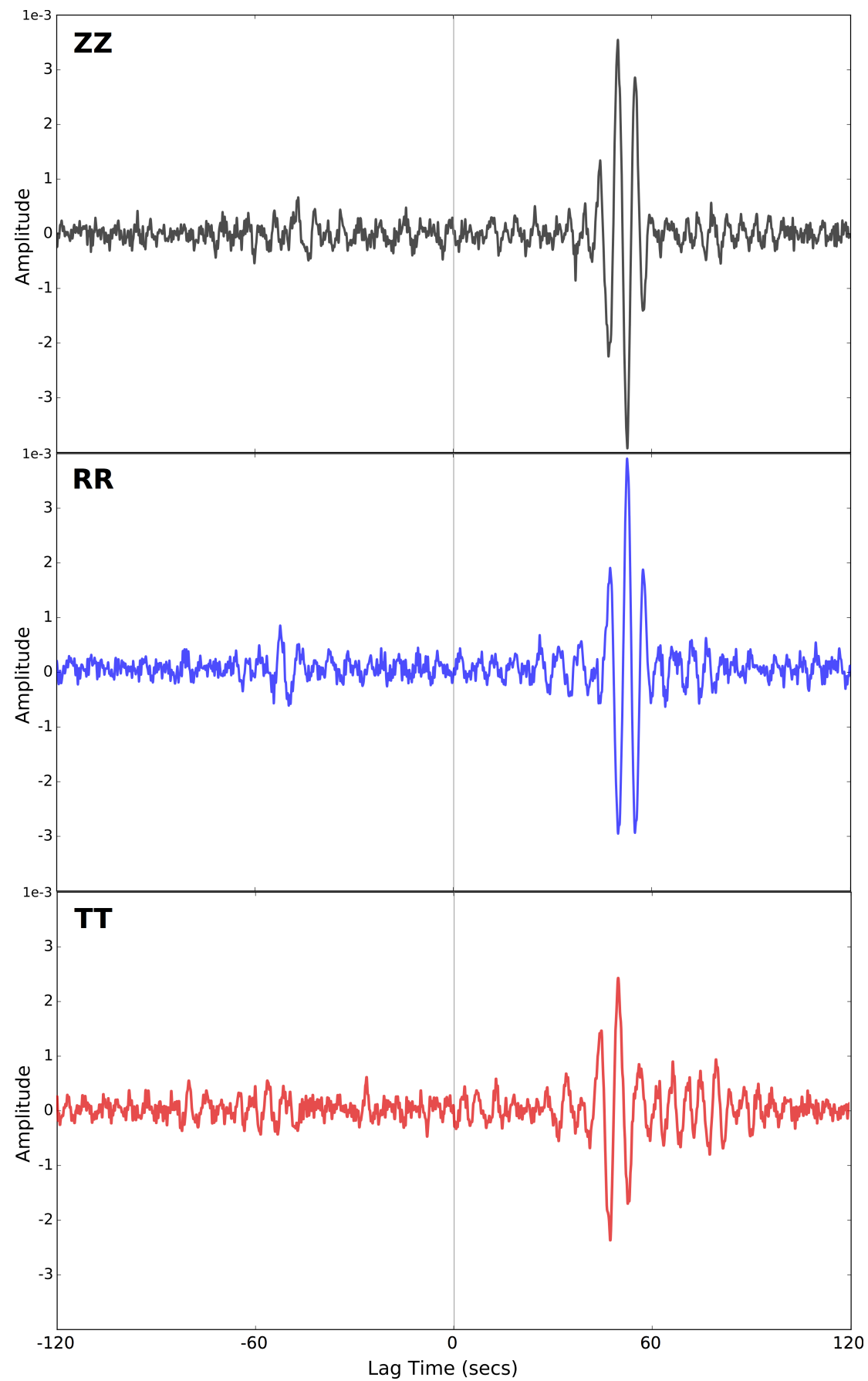

Figure 5.5: Example cross-correlation functions for station pair LBZ-WZ18. For lag times of $-100 \mathrm{~s}-100 \mathrm{~s}$ the cross-correlations for the vertical ZZ (top panel) and horizontal RR and TT (centre and lower panels) components are shown. Figure 5.2 shows the station locations. 


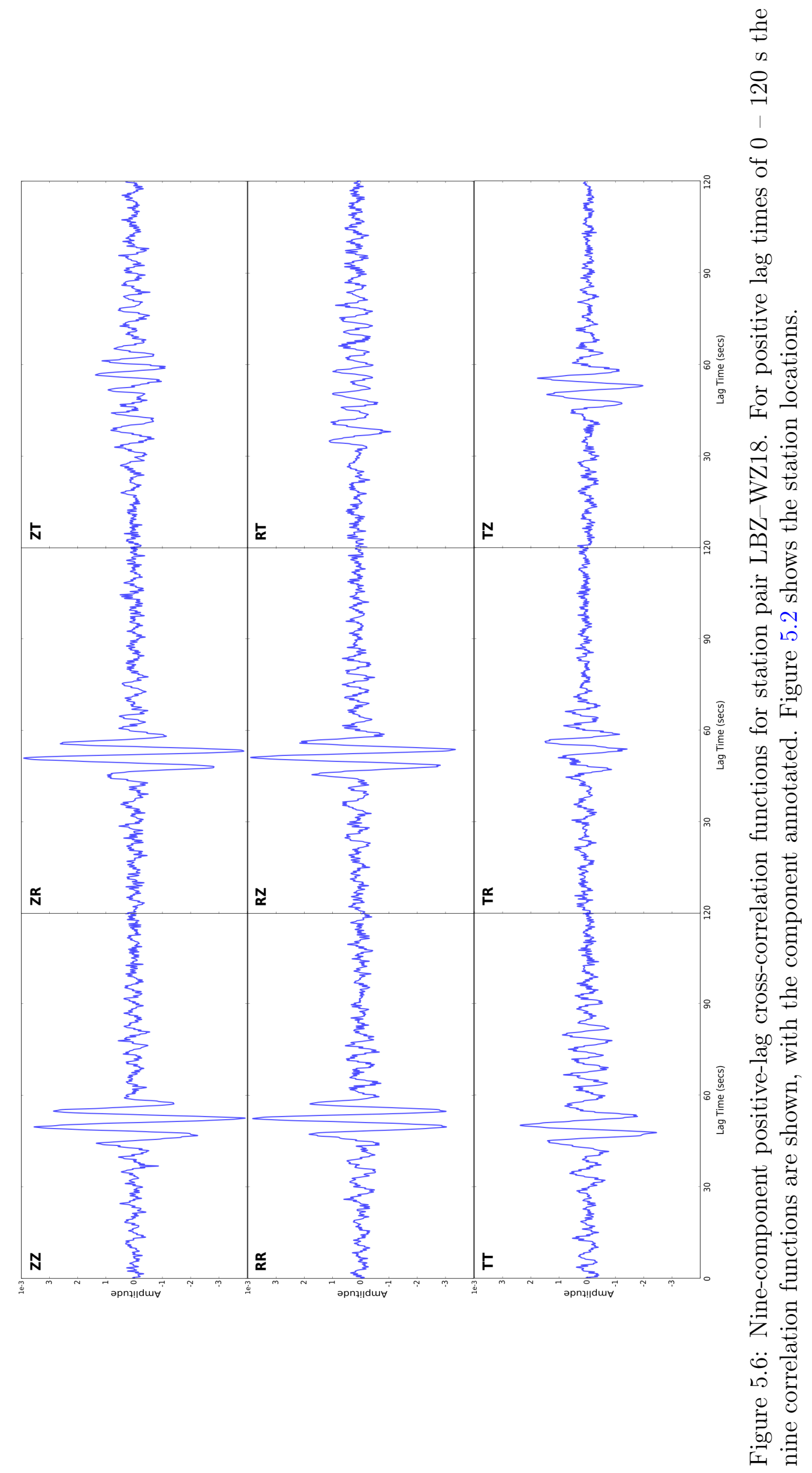




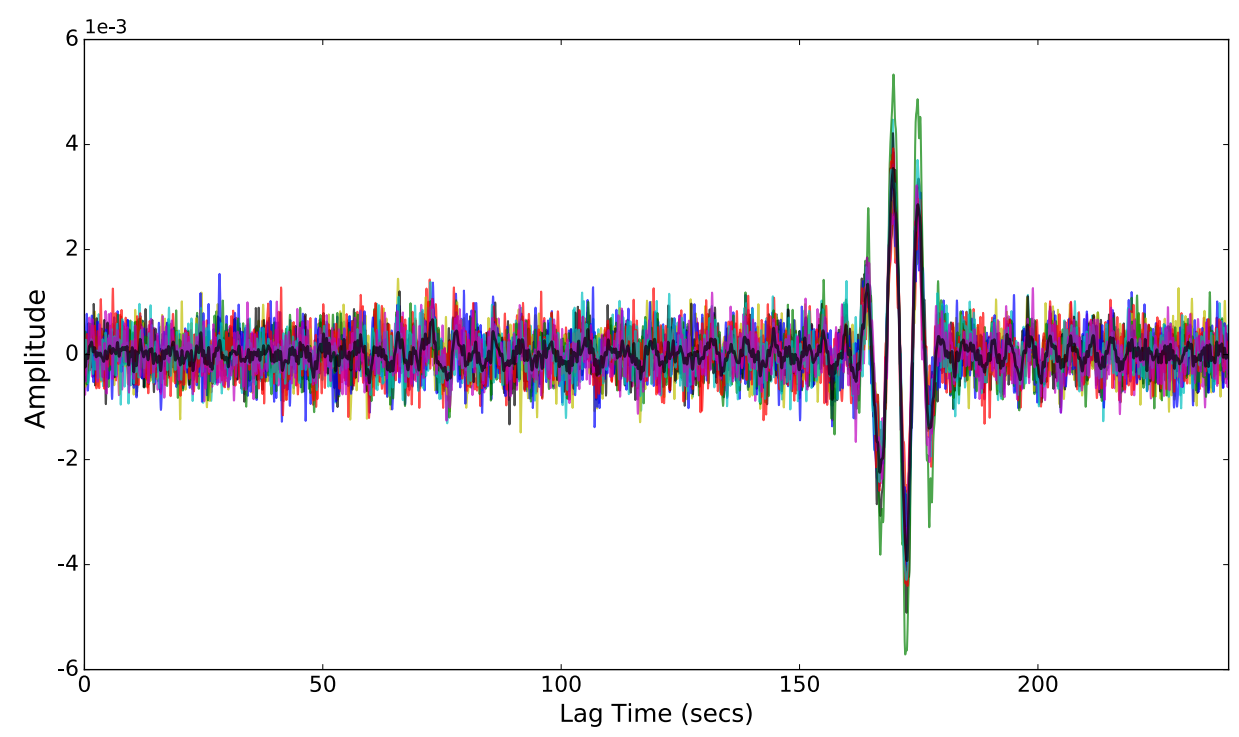

Figure 5.7: Stability of the cross-correlations through time for ZZ functions of station pair LBZ-WZ18. Monthly stacked cross-correlations (coloured) are plotted alongside the reference stack (black).

would be seen on the RR component, and arrive latest on the ZZ component, with the cross-components ZR and RZ recording a superposition of the fundamental and higher-mode. The weakest surface wave arrivals are seen on the radial-transverse cross-components (RT, TR). As there is an arrival, although low amplitude, we can qualitatively conclude that there is some weak anisotropy present in the region. It is unlikely to cause significant discrepancies between later shear-wave models however, as the amplitudes are comparatively low when single component correlations are considered (RR, ZZ, TT).

The stability of the cross-correlations through time is examined for all station pairs. Stacks of 30-day duration for a given pair are compared to each other, and to the overall stack. Figure 5.7 shows this visual check for station pair LBZ-WZ18. When stacked over the whole time period, the surface wave arrival dominates the crosscorrelation and incoherent noise is suppressed at all other lag times.

\subsubsection{Alpine Region Dispersion}

\section{Quality control}

The SNR of symmetric cross-correlations were computed to identify low-quality functions (Figure 5.8, Section 2.6 ). Considering the whole cross-correlation function, between $0.05-2 \mathrm{~Hz}$ (or $0.5-20 \mathrm{~s}$ ), SNRs between 2 and 64 were calculated. Signal- 

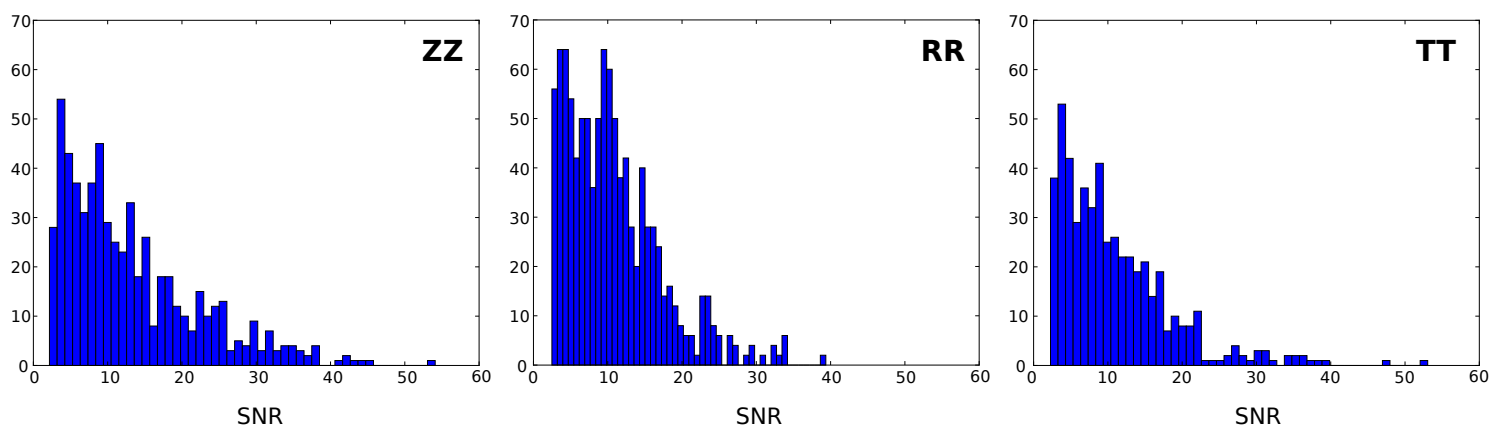

Figure 5.8: Histograms of signal-to-noise ratio for all symmetric cross-correlation functions. SNRs are calculated as outlined in section 2.6. All cross-correlations with a noise ratio less than 8 are discarded.

to-noise ratio as a function of frequency was also examined to determine the most appropriate cut-off period for dispersion analysis. The SNR was highest at $4-8$ $\mathrm{s}$ and quickly decreased at periods greater than $15 \mathrm{~s}$. It was decided that a cutoff period of $12 \mathrm{~s}$ and a SNR threshold of 5 was appropriate for cross-correlation functions to be used in dispersion analyses, to allow high amplitude surface-wave signals and still have a sufficient number of cross-correlations for dispersion and tomographic analyses. This resulted in c. 500 cross-correlations per component going forward.

Detailed examination of the dispersion curves allowed certain stations to be identified that consistently produced poor quality dispersions curves, through velocities anomalously high or low, or erratic picked curves. Such stations were FRAN, MTBA and POCR (Figure 5.2 for station locations). These stations are borehole sensors of the SAMBA network, and so it is possible that the determined orientations are not accurate enough, or the sensors rotated over time. The sensors may not be sufficiently coupled with the borehole and surrounding rock to record the longer period surface waves. It was decided that all cross-correlations including these three stations would be removed from subsequent analyses.

In addition to a SNR threshold of 5 and the removal of low-quality curves from a visual inspection, a wavelength constraint was applied to each curve. Dispersion measurements were removed for periods where the interstation distance was less than 2 wavelengths. This constraint affected cross-correlations between stations of the WIZARD network the most, as these have the shortest interstation paths. Nonetheless in the period band of interest in this study, 4-12 s, the majority of measurements met this criterion.

Short duration cross-correlation stacks of 1 month were processed with the AFTAN method to obtain dispersion curves. The spread of computed velocities for the curves allows an approximation of the uncertainty in the dispersion measurements. 

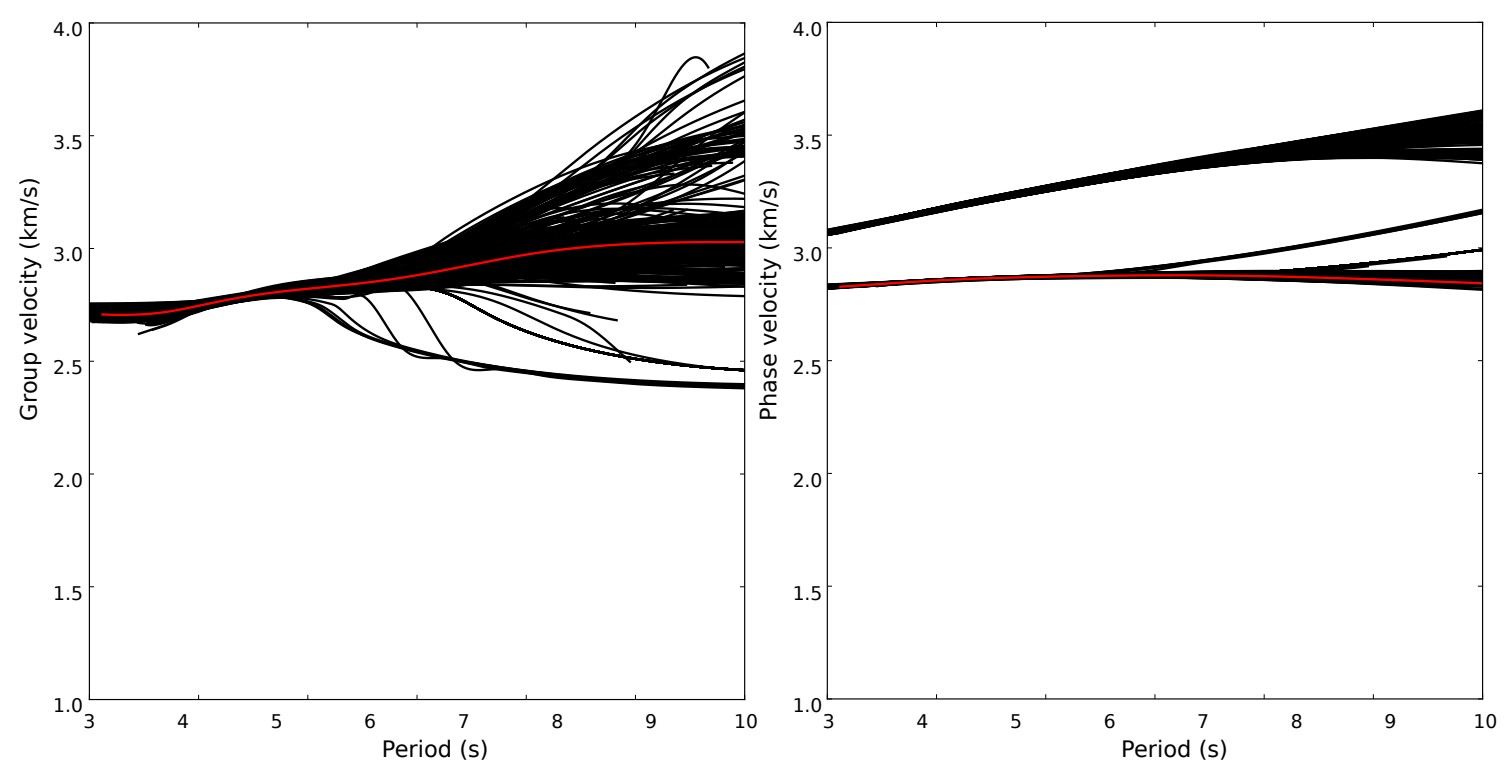

Figure 5.9: Dispersion curves for month-long stacks for station pairs LBZ-WZ18. Group (left) and phase (right) velocity measurements are shown for each monthly vertical component cross-correlation function. The final picked dispersion curve from the stack spanning the whole study period is plotted in red.

An example of vertical component dispersion curves for station pair LBZ-WZ18 is shown in Figure 5.9. The dispersion curves from month-long stacks of crosscorrelations (black) are compared to the full stack's curve (red line). Group velocity curves deviate increasingly from the overall stacked cross-correlation with increasing period. At periods greater than $7 \mathrm{~s}$ the difference in picked velocities reaches 1.0 $\mathrm{km} . \mathrm{s}^{-1}$, however the curve of the full stack appropriately represents the average of the monthly stacks. Phase dispersion measurements typically have a far lower range of velocities, of up to $0.2 \mathrm{~km} . \mathrm{s}^{-1}$. The uncertainties determined for each cross-correlation are incorporated into the following shear-velocity analysis.

\subsubsection{Surface wave tomography}

Following the same procedure in the central Southern Alps as in Section 3.3.4, the dispersion curves that met the quality control procedures are brought forward to the tomographic inversions. A SNR threshold of 5 or greater is required, in addition to the removal of spurious velocity measurements outside the range $1.0 \mathrm{~km} . \mathrm{s}^{-1}$ to 5.0 $\mathrm{km}^{-1}$ and the wavelength constraint is as described previously.

The study area is divided into a $0.1^{\circ} \times 0.1^{\circ}$ grid and following testing of the damping parameters, values of $\alpha=200$ and $\sigma=25$ are selected as appropriate. Tomographic inversions are performed for periods of $4-15 \mathrm{~s}$, however at periods longer than 12 s the path coverage is too sparse to sufficiently resolve the surface velocity models, 

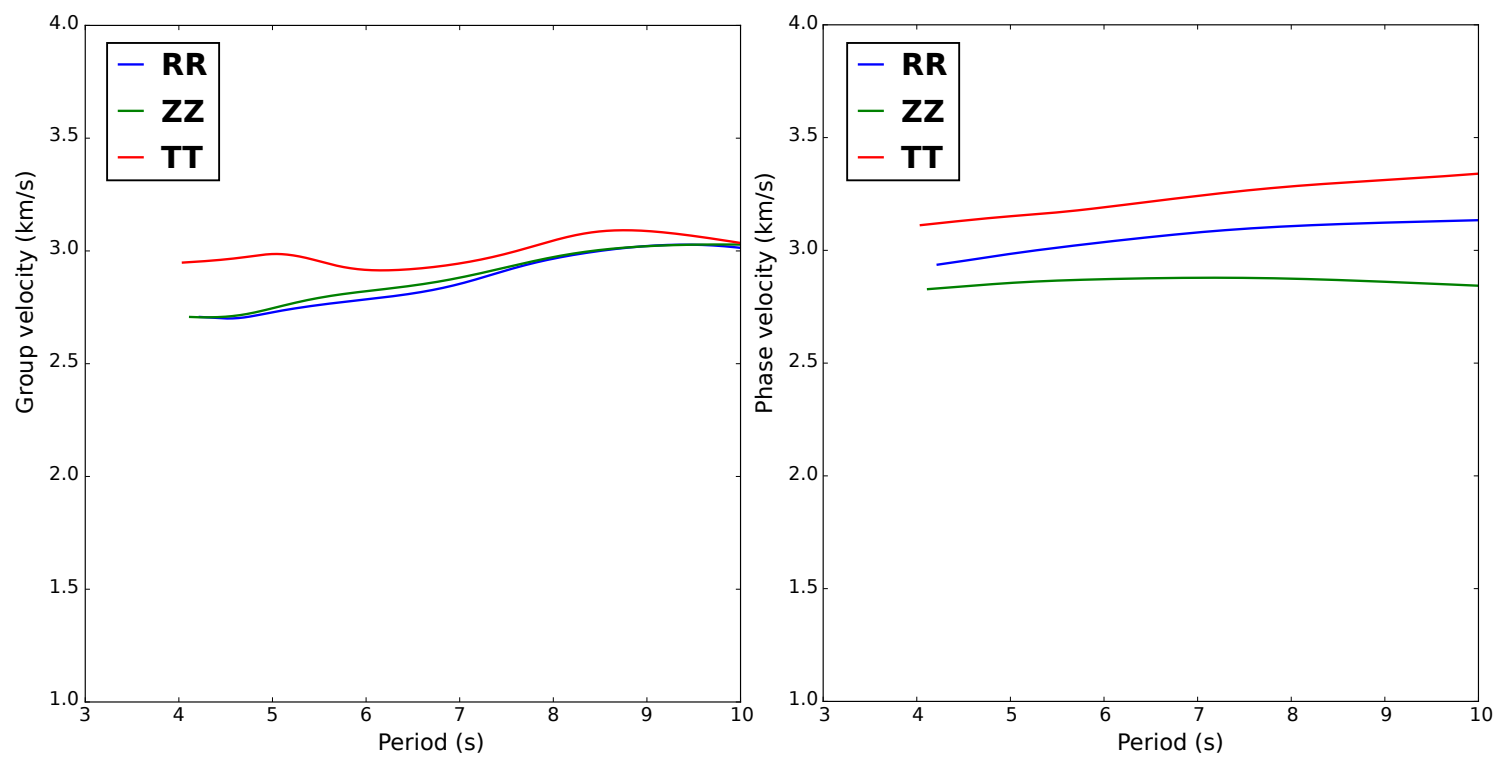

Figure 5.10: Comparison of dispersion curves for RR (blue), ZZ (green) and TT (red) components. For periods of $4-10 \mathrm{~s}$ the group and phase dispersion curves are shown for example station pair LBZ-WZ18.

and so they are not presented in this study.

The path coverage for $R R$ group dispersion curves is shown in Figure 5.11. As expected the densest coverage is to the north of the area, within the WIZARD network. There is some bias from paths trending NE-SW along the surface trace of the Alpine Fault, however there are still over 100 station pairs throughout the region at $12 \mathrm{~s}$, and up to 390 at $5 \mathrm{~s}$. Figure 5.12 illustrates this directional bias, with high azimuthal coverage to the northeast of the region, at the location of the WIZARD array. To the south of $-43.9^{\circ}$ there are only 3 seismic stations; LBZ, JCZ and SOLU (see Figure 5.2 for locations). Most paths from these stations strike northeast, along strike of the fault, resulting in an azimuthal coverage as low as $20^{\circ}$ in some locations.

The output of the tomographic resolution matrix is shown in Figure 5.13. As explained in section 3.3.4, the resolution is defined as the distance at which a $\delta$-shaped perturbation in the velocity model at each nodal point can be fully resolved, to a minimum distance of twice the inversion grid spacing. In this study as in Chapter 3 a grid size of $0.1^{\circ} \times 0.1^{\circ}$ allows for a minimum spatial resolution of approximately $22 \mathrm{~km}$. The spatial resolution is similar at all periods (Figure 5.13) and is high throughout the region. As expected, the highest resolutions of $\sim 22$ are located to the northeast, but resolutions do not exceed $35 \mathrm{~km}$ even where path coverage is sparse, to the southwest of the study region. The model space is limited to the region within which spatial resolution is less than $40 \mathrm{~km}$. 


\subsubsection{Shear wave inversion}

Dispersion curves at each nodal point are obtained by interpolating between tomography models, as in section 3.3.5. This results in dispersion curves every $0.1^{\circ}$, which are inverted for 1-D shear velocity models. Thorough testing of the inversion parameterisation is undertaken to obtain the most appropriate starting model for the region. To ensure that the maximum amount of the parameter space is searched during inversions, the shear-velocities are allowed to vary over wide ranges. A threelayer over a half-space model was implemented, with a thin surface-layer (Table 5.2). During testing this upper layer was allowed to have a very large range of between 0.1 - $3.0 \mathrm{~km} . \mathrm{s}^{-1}$. It was possible to resolve a low-velocity surface layer in near-coastal locations and to the east of the Alpine ranges, but it was not always present in final models within the southern Alps. Density and $V_{P}$ were set to vary, dependent on $\mathrm{V}_{S}$, and allowed to vary over wide ranges.

The dispersion curves between 5 and 12 s periods were converted to slowness vs. frequency and resampled to have 50 points in log-log space. This smoothed the curves, improving the inversions and allowing better model fits to the data. At each location in the gridded region several sets of inversions of 50000 iterations were performed. This ensures that the parameter space is sufficiently searched and models do not get trapped in local misfit minima. Misfit thresholds of 0.05 for single mode inversions is allowed. The preferred model is calculated from a weighted average of all models with misfits below the chosen thresholds.

Table 5.2: Southern Alps shear-wave inversion initial model parameterisation

\begin{tabular}{cccc}
\hline $\mathbf{V}_{P}\left(\mathrm{~km} . \mathbf{s}^{-1}\right)$ & $\mathbf{V}_{S}\left(\mathrm{~km} . \mathbf{s}^{-1}\right)$ & Depth to layer base $(\mathrm{km})$ & Density $\left(\mathrm{kg} . \mathrm{cm}^{3}\right)$ \\
\hline $1.0-5.0$ & $1.0-3.0$ & $0.1-3.0$ & $2.0-4.0$ \\
$1.0-7.0$ & $1.9-4.0$ & $1.0-8.0$ & $2.0-4.0$ \\
$3.0-8.0$ & $2.5-4.5$ & $5.0-12.0$ & $2.0-4.0$ \\
$4.0-9.2$ & $3.0-5.0$ & - & $2.0-4.0$ \\
\hline
\end{tabular}

\subsection{Results}

Surface wave velocity maps for 5 - 12 s periods are shown in Figures 5.14 to 5.19. The region of high spatial resolution below $40 \mathrm{~km}$ is outlined by the white contour and velocities within are brightened. A scale bar for $20 \mathrm{~km}$ is shown as the minimum possible size for real features to be resolved in the inversions. The surface expression of the Alpine Fault is indicated by the dashed grey line on each map. 


\subsubsection{Rayleigh and Love Group Velocity Maps}

Rayleigh wave group velocity maps from vertical cross-correlations are presented in Figure 5.14. At 5-7 s periods there is a SW-NE trending high velocity zone of $3.4-$ $3.7 \mathrm{~km} . \mathrm{s}^{-1}$, with the same strike as the Alpine Fault, underneath the Southern Alps mountain range. This is flanked to either side by zones of lower velocities between $2.4 \mathrm{~km} . \mathrm{s}^{-1}$ to $2.7 \mathrm{~km} . \mathrm{s}^{-1}$. The contrast between these regions is sharp, with the Alpine Fault approximately marking the boundary to the northwest of the high velocity zone. At longer periods the range of modelled velocities is less, with the background lower velocities increasing to approximately $3.0 \mathrm{~km} . \mathrm{s}^{-1}$. There is a small low-velocity feature to the northeast of the model region, at $\sim-43.5,170.8$. There are no seismic stations in the area, but it is crossed by all station paths travelling from stations of the WIZARD array to RPZ.

Figure 5.15 also shows Rayleigh group velocities, however they are the results of inverting dispersion curves of RR cross-correlations. For periods of $5-7 \mathrm{~s}$ the background velocities range from $2.4 \mathrm{~km} . \mathrm{s}^{-1}$ to $2.8 \mathrm{~km} . \mathrm{s}^{-1}$, highlighting the Southern Alps. The high velocity zone to the south of the Alpine Fault's surface trace is approximately $40-50 \mathrm{~km}$ wide, striking NE-SW with velocities of $3.2-3.6 \mathrm{~km} . \mathrm{s}^{-1}$. The low velocity anomaly seen for the ZZ Rayleigh maps is also present in the RR Rayleigh group tomography.

Love group velocities show a large low velocity region striking N-S through the centre of the study region, especially at short periods (Figure 5.16). There are regions of higher velocities between $3.0-3.6 \mathrm{~km} . \mathrm{s}^{-1}$. Looking at the Love wave map for 10 and $12 \mathrm{~s}$, high and low velocities appear patchy and somewhat smeared, creating narrow bands E-W and N-S. These features are on the same order of the spatial resolution in the region (Figure 5.13). They are in regions of low path coverage (Figure 5.11), radiating outwards from seismic stations LBZ and RPZ (see Figure 5.2 for station locations), with no other stations within at least $50 \mathrm{~km}$ of either. It is therefore likely they are artefacts of the inversion procedure. 


\subsubsection{Rayleigh and Love Phase Velocity Maps}

Overall, the phase velocity maps at periods $5-12 \mathrm{~s}$ exhibit faster velocities than group at equivalent periods, as expected. Rayleigh phase velocities from vertical components show relatively homogeneous velocity models throughout the region, ranging between $2.9-3.4 \mathrm{~km} . \mathrm{s}^{-1}$ for $5-8$ s periods (Figure 5.17). The boundary between high and low velocities at the Alpine Fault is not as clear as in Rayleigh group maps (Figure 5.14). The low velocity patch seen in Rayleigh group velocities to the northeast of the model space is present in the phase maps. Unexpectedly it decreases in velocity with increasing period, to less than $2.5 \mathrm{~km} . \mathrm{s}^{-1}$ at $12 \mathrm{~s}$. This feature does not appear in phase velocity maps from horizontal RR components, which exhibit far more homogeneous velocities with no striking features (Figure 5.18). Rayleigh phase velocities to the south of the region reach $3.7 \mathrm{~km} . \mathrm{s}^{-1}$.

Comparing the Rayleigh wave velocity maps obtained through inversion of vertical ZZ and horizontal RR dispersion curves is important as it gives an estimation of error and an indication of the success and suitability of the quality controls placed throughout processing. It can also give confidence that the horizontal component raw data were accurately reoriented. The similarity of the Rayleigh group velocity maps allows confidence in the reoriented data and subsequent models. The anomalous low velocity zone in the ZZ Rayleigh phase maps suggests some anomalous dispersion measurements, particularly at long periods. These are likely to have come from cross-correlations involving station RPZ. This station is located on the southeastern extent of the Southern Alps, bordering the Canterbury Plains.

Love phase velocities throughout the region are faster than Rayleigh phase at equivalent periods. For periods $5-8 \mathrm{~s}$ velocities range from $3.1 \mathrm{~km} . \mathrm{s}^{-1}$ to $3.5 \mathrm{~km} . \mathrm{s}^{-1}$. Velocity variations appear patchy and do not appear to correspond to the Alpine Fault or Southern Alps mountains (Figure 5.19).

\subsubsection{Sensitivity Kernels}

Depth sensitivity kernels for an average regional velocity model are calculated to give an indication of the depths of the major features visible in the tomographic period maps. The sensitivity is given by the partial derivates of phase and group velocities with respect to shear wave velocities as a function of depth. The average regional model is given in Appendix B. The resulting kernels are shown in Figure 5.20 and are largely comparable to those produced for the Canterbury Plains in Figure 3.28. Curves are given for periods $5-10 \mathrm{~s}$ for group and phase velocities (left and right panels) for Rayleigh and Love fundamental modes (top and bottom panels). The 
only obvious difference between the sensitivities of the Alps and Canterbury is the Rayleigh waves are slightly less sensitive to shear velocities between $3-6 \mathrm{~km}$ in the Alps. Group velocities have higher sensitivities than phase velocities, but are not sensitive to as large depth ranges. As period increases, surface wave sensitivities are of lower amplitudes at equivalent depths until $3-7 \mathrm{~km}$ for group velocities, after which longer periods become more sensitive to shear velocities. The same trend is seen after $5-7 \mathrm{~km}$ depth for phase velocities. Love waves are again most sensitive to the uppermost $3 \mathrm{~km}$, but exhibit a second sensitivity peak at approximately 4 $5 \mathrm{~km}$ depth. Rayleigh group and phase velocities are sensitive to the very shallow subsurface (for depths less than $\sim 1 \mathrm{~km}$ ), and show a stronger peak at $4-6 \mathrm{~km}$.

\subsubsection{Shear velocity Models}

Higher confidence can in general be placed in the phase velocity dispersion measurements since they had far less variation in the month-long cross-correlations, and therefore uncertainties in the overall stacked dispersion measurements are lower. Additionally, cross-correlations of the horizontal radial-radial component had higher signal-to-noise ratios than vertical components, resulting in a denser path coverage in tomographic models. One-dimensional $\mathrm{V}_{S}$ models are therefore obtained from Love and Rayleigh phase dispersion measurements obtained from TT and RR crosscorrelation components. The models presented show the preferred profile from all inversions giving misfits less than 0.05. This preferred is calculated as in Section 3.4.4 by a weighted average of all accepted models.

Figure 5.22 presents the models obtained at 6 locations within the central Southern Alps (Figure 5.21 highlights points $\mathrm{A}-\mathrm{F}$ ). Locations A and D are to the northwest of the Alpine Fault, close to the coast. B and E lie within the Southern Alps mountain range, with $\mathrm{B}$ situated within the WIZARD array, and $\mathrm{E}$ to the south, close to seismic station SOLU. C and F lie on the southeastern foothills of the Alps, to the edge of the tomographic inversion region. C additionally lies at the centre of the velocity low apparent in Rayleigh-wave tomographic models (Figures 5.14 $5.18)$.

Location A consists of a thin layer up to $1 \mathrm{~km}$ thick with low velocities of 0.7 $1.1 \mathrm{~km} . \mathrm{s}^{-1}$ overlying a basement rock with velocities between $1.8-2.6 \mathrm{~km} . \mathrm{s}^{-1}$. At approximately $5 \mathrm{~km}$ depth, a fast layer of $3.3 \mathrm{kms}^{-1}$ begins. Location D appears less constrained at the shallow subsurface below $2 \mathrm{~km}$ depth. The preferred model has a slow subsurface layer present; however it is extremely thin, possibly below the minimum thickness that can confidently be resolved. The velocity of this layer is somewhat faster than at A, at $1.5 \mathrm{~km} . \mathrm{s}^{-1}$. A faster layer of $2.7-3.2 \mathrm{~km} . \mathrm{s}^{-1}$ begins 
at a much shallower depth of approximately $1.6-2.0 \mathrm{~km}$.

Locations B and E give extremely homogeneous shear-wave velocity models. Aside from a very thin low-velocity near-surface layer up to $300 \mathrm{~m}$ thick, the shear velocity near constant with depth. Both locations show a velocity of $3.1-3.4 \mathrm{~km} . \mathrm{s}^{-1}$ over most of the model space. The models are well constrained at depth, especially below $2 \mathrm{~km}$.

$\mathrm{C}$ and $\mathrm{F}$ both show a slow surface layer up to $500 \mathrm{~m}$ thick. At $\mathrm{C}$ velocities increase to $2.3-2.6 \mathrm{~km} . \mathrm{s}^{-1}$ for $3 \mathrm{~km}$, although the model is not well constrained at this depth. Below $3.5 \mathrm{~km}, \mathrm{~V}_{S}$ further increases to $3.1 \mathrm{~km} \cdot \mathrm{s}^{-1}$. Location $\mathrm{F}$ is poorly constrained in the uppermost $3 \mathrm{~km}$ but shows velocities of $3.1-3.4 \mathrm{~km} . \mathrm{s}^{-1}$ below this depth.

To give an indication of the variability of possible velocity models at a given location, and to assess depth resolution, different surface wave phases are inverted and compared. Figure 5.23 presents the preferred shear-velocity models derived from Love and Rayleigh dispersion measurements, for location B and F, which are situated within and to the southwest of the Southern Alps mountains. For both locations the Love- and Rayleigh-derived models have good agreement. Velocities at depth are consistent, although the Love-derived are less constrained than the Rayleigh ones below $2 \mathrm{~km}$ for location $\mathrm{F}$. The shallow layers are better resolved for both locations by inversions of Love dispersion. At B, a slow $700 \mathrm{~m}$ thick layer with velocity $0.6-0.8 \mathrm{~km} . \mathrm{s}^{-1}$ at the surface. Location $\mathrm{F}$ shows a very slow $200 \mathrm{~m}$ thick near-surface layer, as with the Rayleigh phase model, with velocities of only several hundred metres per second. This overlies a layer approximately $2 \mathrm{k} \mathrm{m}$ thick with $\mathrm{V}_{S}=2.0-2.4 \mathrm{~km} . \mathrm{s}^{-1}$, which is seen in the Rayleigh models, but is more poorly constrained.

\subsection{Discussion}

Surface-wave tomography maps and $1-\mathrm{D} \mathrm{V}_{S}$ models were successfully obtained for the central Southern Alps. Within the Alpine mountain range, elevated seismic velocities and homogenous shear-velocity profiles with depth are attributed to the presence of little-to-no sedimentary cover overlying the schists of the Alps. This feature is seen in $\mathrm{V}_{P}$ and $\mathrm{V}_{S}$ models from studies using other methods, however the models obtained in this study slightly underestimate $\mathrm{V}_{S}$ in this area compared to the other studies. ?Bourguignon et al. (2015) both obtain shear-velocities between $3.5-3.8 \mathrm{~km} . \mathrm{s}^{-1}$, slightly higher than the $3.3-3.5 \mathrm{~km} . \mathrm{s}^{-1}$. 
Inversions of Love and Rayleigh phase velocities for $\mathrm{V}_{S}$ have excellent agreement at depth, with models extremely well constrained below $2-3 \mathrm{~km}$ (Figure 5.23). On the footwall of the Alpine Fault, a thin layer up to a maximum of $1 \mathrm{~km}$ thick with slow velocities below $1.0 \mathrm{~km} . \mathrm{s}^{-1}$ exists. Bourguignon et al. (2015); ? do not resolve the velocity model at the surface, but at $3 \mathrm{~km}$ show velocities of $2.4-2.7 \mathrm{~km} . \mathrm{s}^{-1}$, in agreement with location A. ? additionally shows some thinning of the uppermost layers below $3 \mathrm{~km}$ ) on the footwall of the Alpine Fault, for points located to the south of the study region. It is possible that the same feature is observed when comparing locations $\mathrm{A}$ and D.

To the southeast of the Southern Alps, a low velocity anomaly is present to the north of the study region. In addition to the thin low velocity layer at the immediate subsurface, which is due to sedimentary cover thickening from the foothills of the Alps and towards the eastern edge of the Canterbury Plains, a layer $4 \mathrm{~km}$ thick is modelled at Point C. The average velocity within this layer is $2.2-2.6 \mathrm{~km} \cdot \mathrm{s}^{-1}$. This anomaly is most likely due to poor quality dispersion measurements for station pairs including RPZ. RPZ is the only station to the northeast of the study region, and so there is likely smearing in the tomographic inversions and the low velocities measured are an artefact of this. The location of RPZ at the edge of the Canterbury Plains, which has a thicker sedimentary cover than within the Alps, is likely further biasing the dispersion measurements and as a result the shear-wave velocity inversions.

Points D - F lie in the southern side of the study region, where path coverage is low and spatial resolution is worst. It was still possible to record spatial resolutions within this region as low as $40 \mathrm{~km}$, and so we can have reasonable confidence in the models obtained here. Feenstra et al. (2016) performed $V_{P}$ tomography from earthquake arrival times, using the WIZARD array and SAMBA stations in conjunction with others. Features observed by Feenstra et al. (2016) agree well with those modelled in this study, with the lowest velocities to the northwest of the Alpine fault. The Southern Alps mountains were clearly defined by a high-velocity zone extending the length of the West Coast.

For inversions of both Rayleigh and Love phase dispersion curves, models with acceptable misfits are well constrained. Rayleigh wave derived models appear to be less well constrained than Love in the uppermost $2-5 \mathrm{~km}$ for all locations. This is due to the surface waves exhibiting shear wave sensitivity at differing depths, as investigated in Section 5.4.3. Inversions of Rayleigh and Love phase velocities allowed the $\mathrm{V}_{S}$ models to be well constrained at differing depths. Love waves were able to model the uppermost $2-3 \mathrm{~km}$ with low model misfits and good convergence, and Rayleigh waves were best constrained below $3 \mathrm{~km}$. These observations are in good agreement with the depth sensitivity kernels and so allow high confidence that the 
input velocity models used throughout processing were appropriate. Although joint inversions were unsuccessful due to differing strengths of surface wave sensitivities, and the inability to weight input dispersion data accordingly, it has been shown that there is great merit in using several surface wave phases separately to obtain shear-velocity models, and they should be examined together to locate the depths of highest model confidence. 

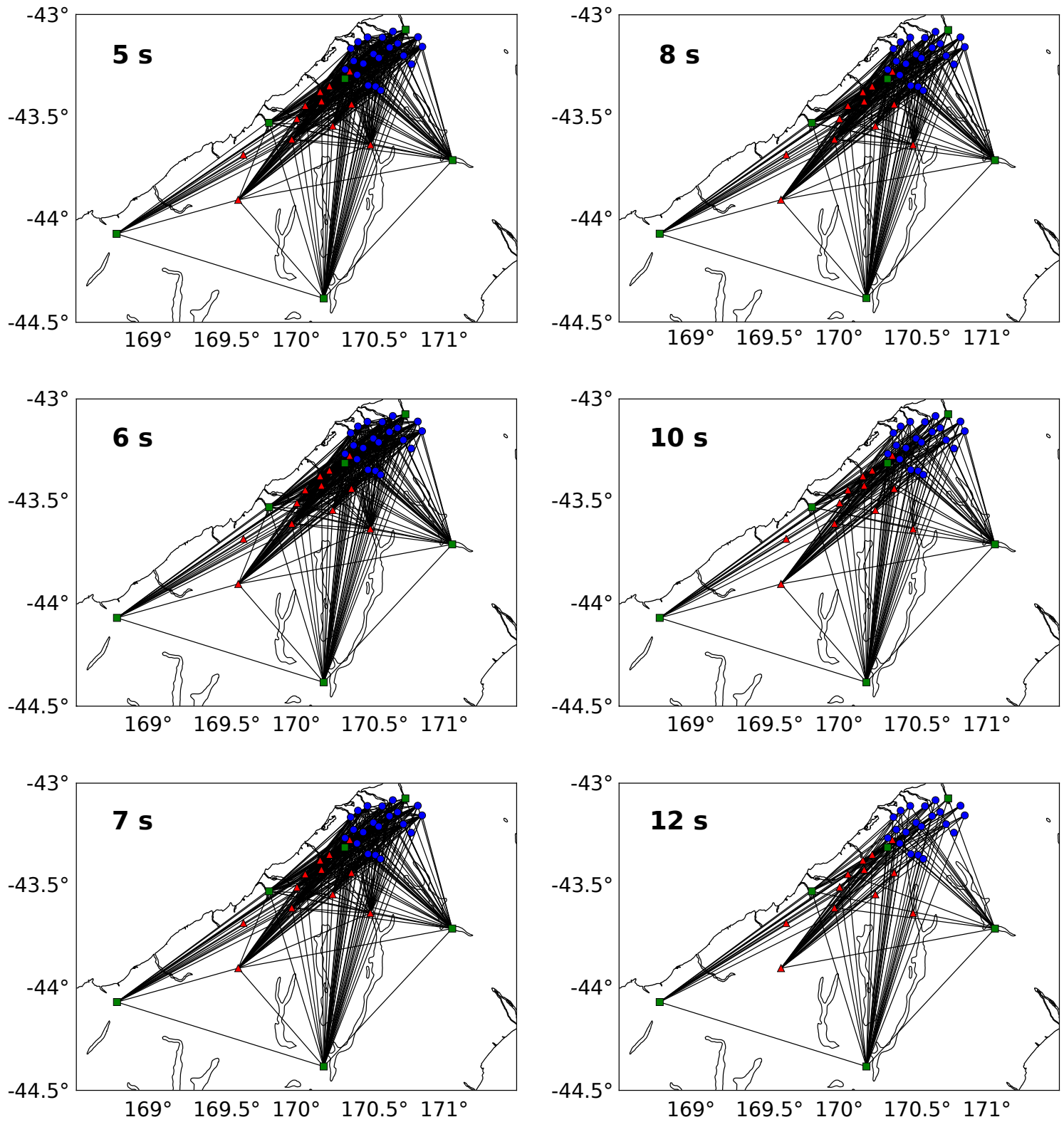

Figure 5.11: Interstation path coverage for the central Southern Alps region, as a function of period. Each available interstation path is plotted for periods $5-12 \mathrm{~s}$. It is seen that for increasing periods, fewer measurements are allowed for tomographic inversions, due to measurements not meeting quality control measures. 

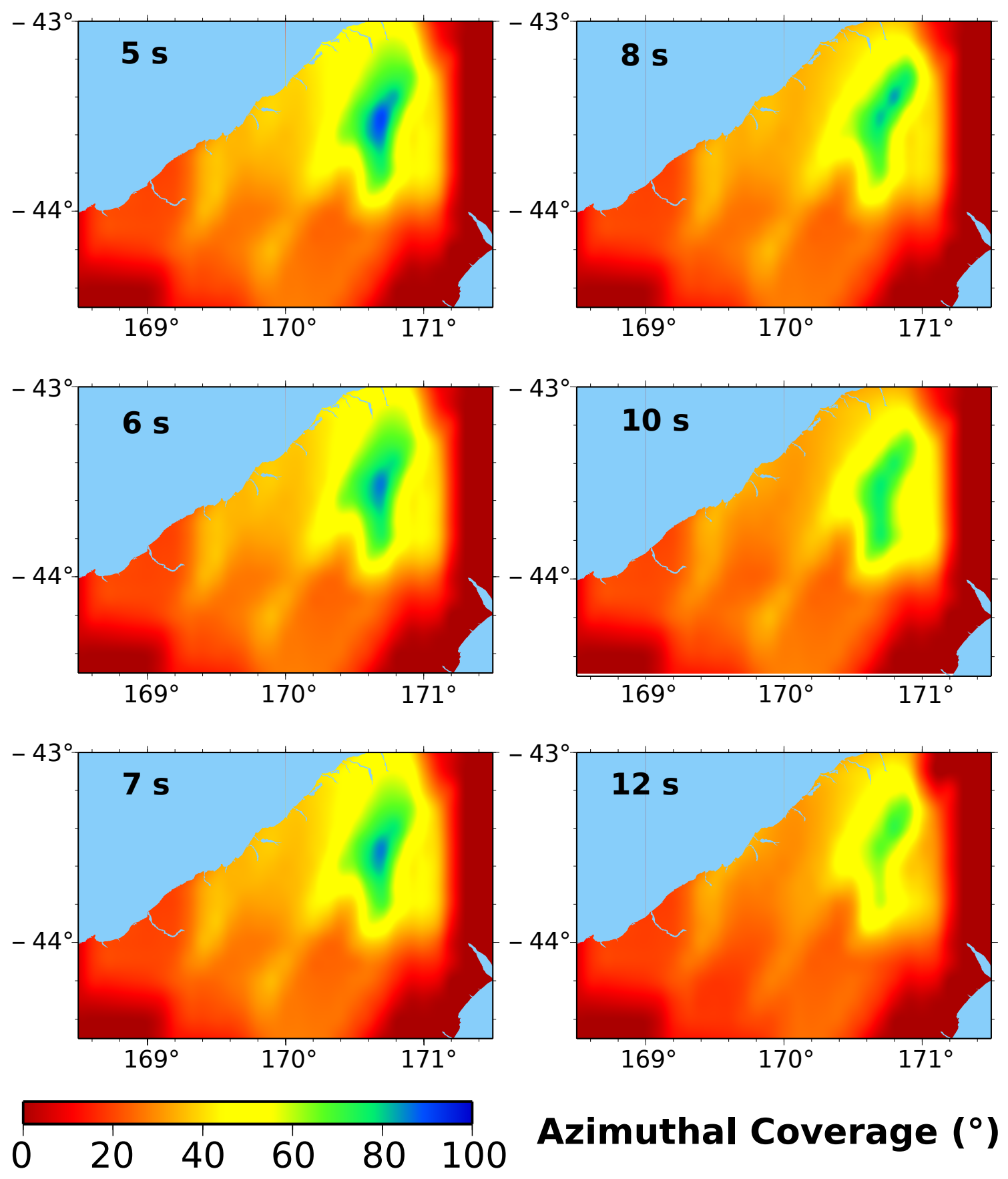

Figure 5.12: The azimuthal path coverage at each nodal point in the tomographic inversions is calculated for periods $5-12 \mathrm{~s}$. 

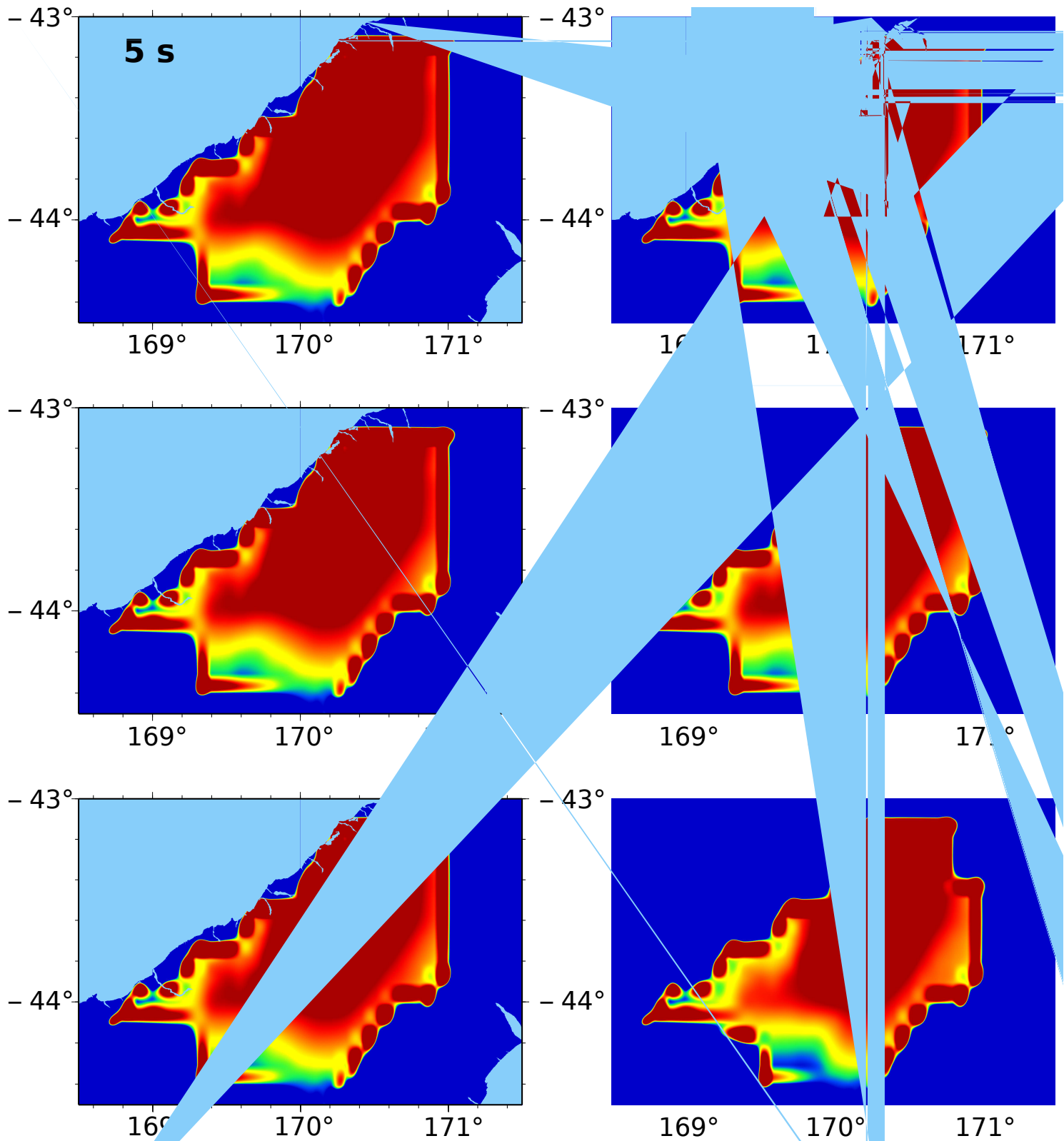

Figure 5.13: Spatial resolution analysis of the Southern Alps tomography maps. For periods of $5-12 \mathrm{~s}$, the spatial resolution is calculated as described in Section 5.3 .3 and mapped, to a maximum spatial resolution of $40 \mathrm{~km}$. The resolution in kilometres describes the minimum distance at which a $\delta$-perturbation can be fully resolved. Resolutions at points greater than $40 \mathrm{~km}$ have been set at this maximum in the figure, to easily visualise the regions of good resolution. 

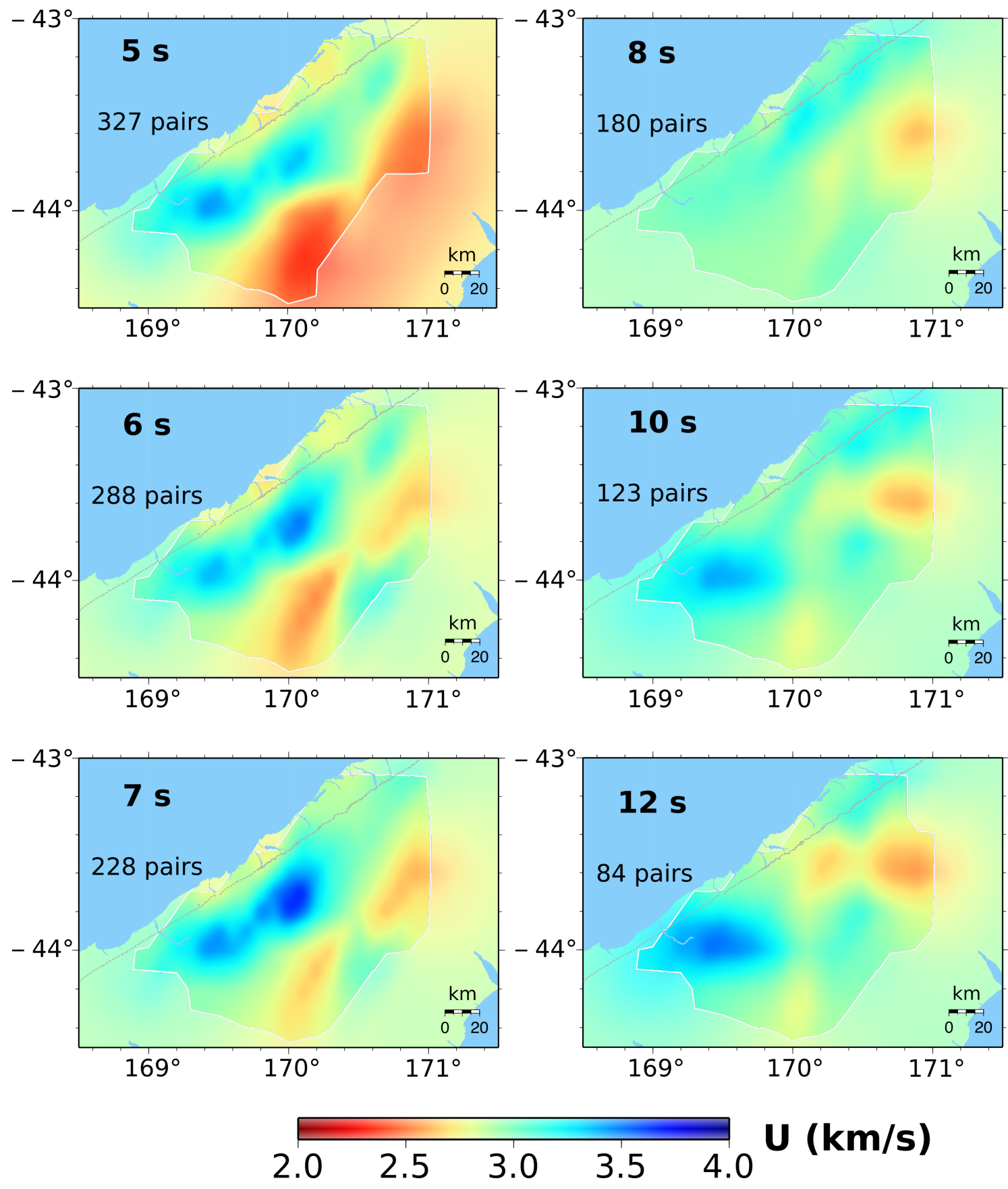

Figure 5.14: Rayleigh wave group velocity maps for 5 to $12 \mathrm{~s}$ periods from ZZ crosscorrelations. Bold colours denote the model region where spatial resolution is $40 \mathrm{~km}$ or lower, with white contours outlining this region and colours outside the region masked. The surface expression of the Alpine Fault is indicated by the dashed grey line. 

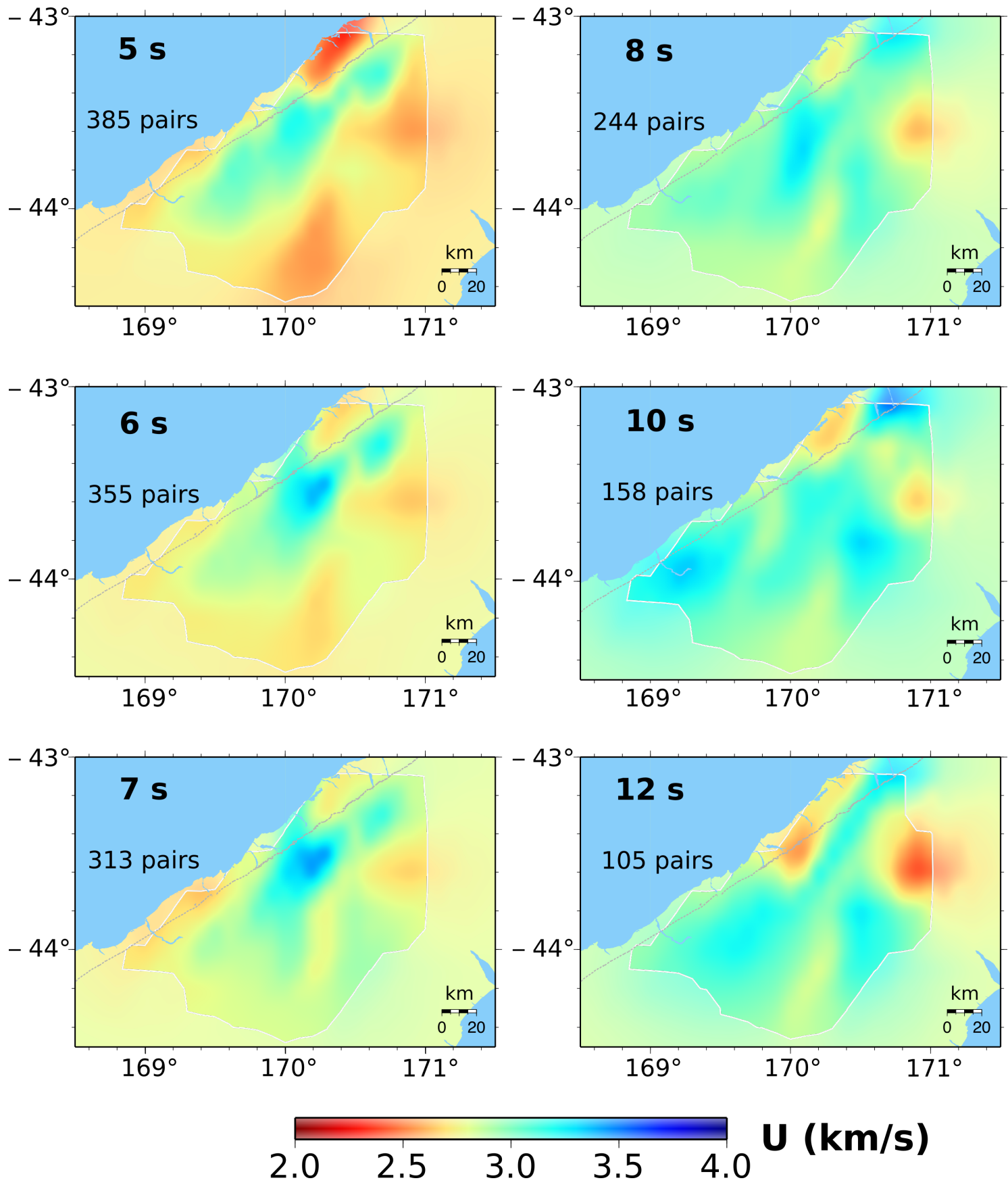

Figure 5.15: Rayleigh wave group velocity maps for 5 to $12 \mathrm{~s}$ periods from RR crosscorrelations. Bold colours denote the model region where spatial resolution is $40 \mathrm{~km}$ or lower, with white contours outlining this region and colours outside the region masked. The surface expression of the Alpine Fault is indicated by the dashed grey line. 

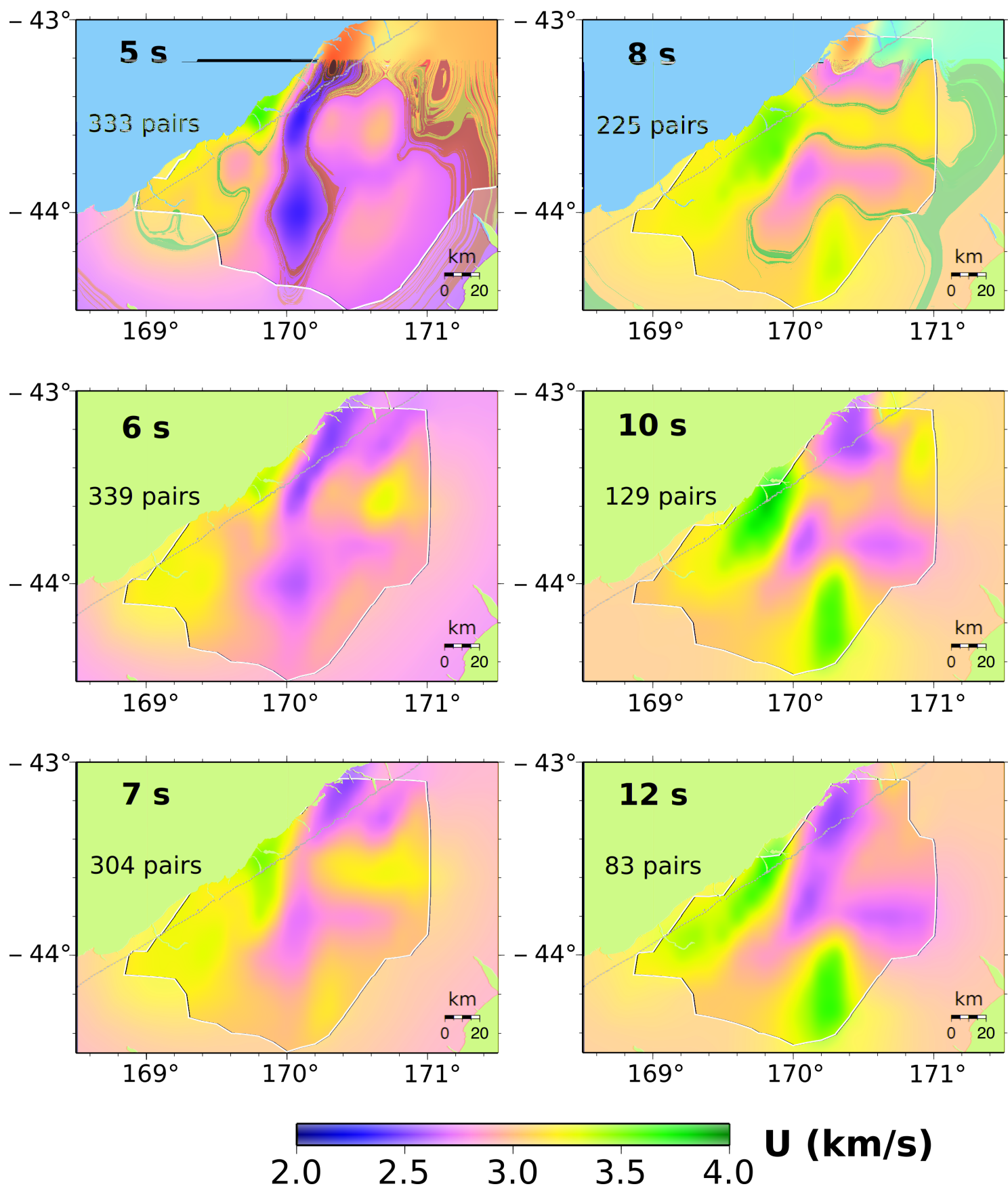

Figure 5.16: Love wave group velocity maps for 5 to $12 \mathrm{~s}$ periods from TT crosscorrelations. Bold colours denote the model region where spatial resolution is $40 \mathrm{~km}$ or lower, with white contours outlining this region and colours outside the region masked. The surface expression of the Alpine Fault is indicated by the dashed grey line. 

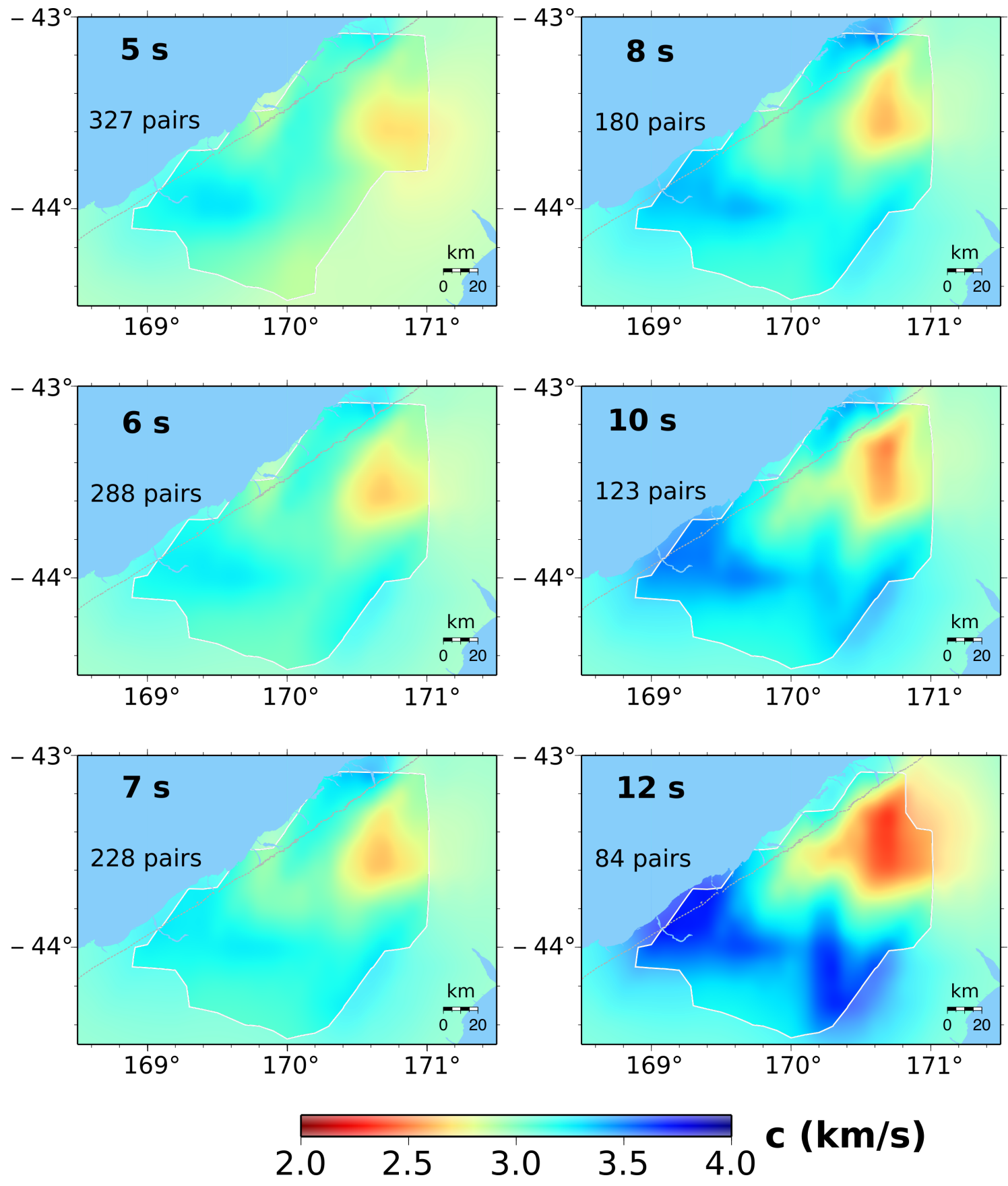

Figure 5.17: Rayleigh wave phase velocity maps for 5 to $12 \mathrm{~s}$ periods from ZZ crosscorrelations. Bold colours denote the model region where spatial resolution is $40 \mathrm{~km}$ or lower, with white contours outlining this region and colours outside the region masked. The surface expression of the Alpine Fault is indicated by the dashed grey line. 

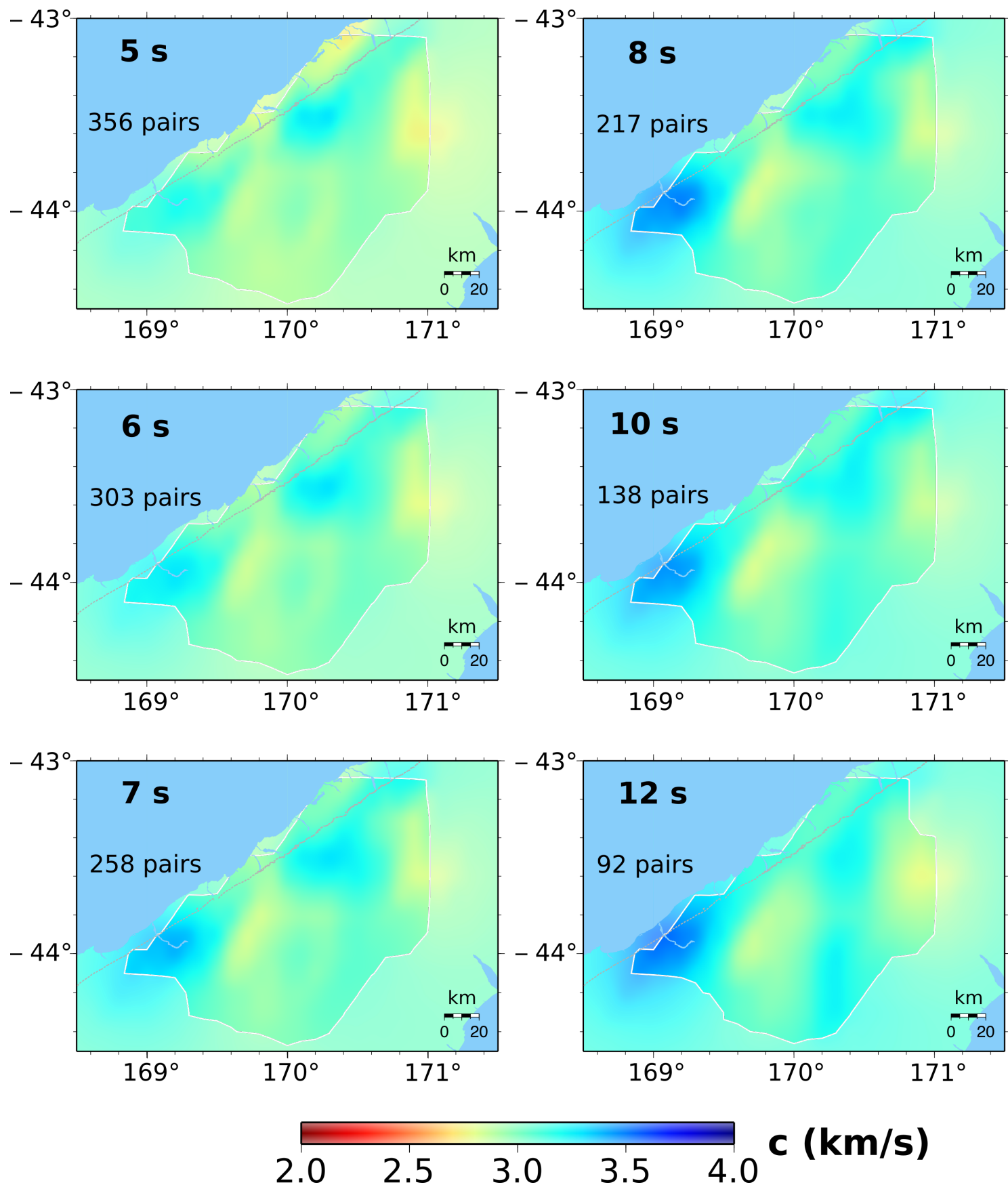

Figure 5.18: Rayleigh wave phase velocity maps for 5 to $12 \mathrm{~s}$ periods from RR crosscorrelations. Bold colours denote the model region where spatial resolution is $40 \mathrm{~km}$ or lower, with white contours outlining this region and colours outside the region masked. The surface expression of the Alpine Fault is indicated by the dashed grey line. 

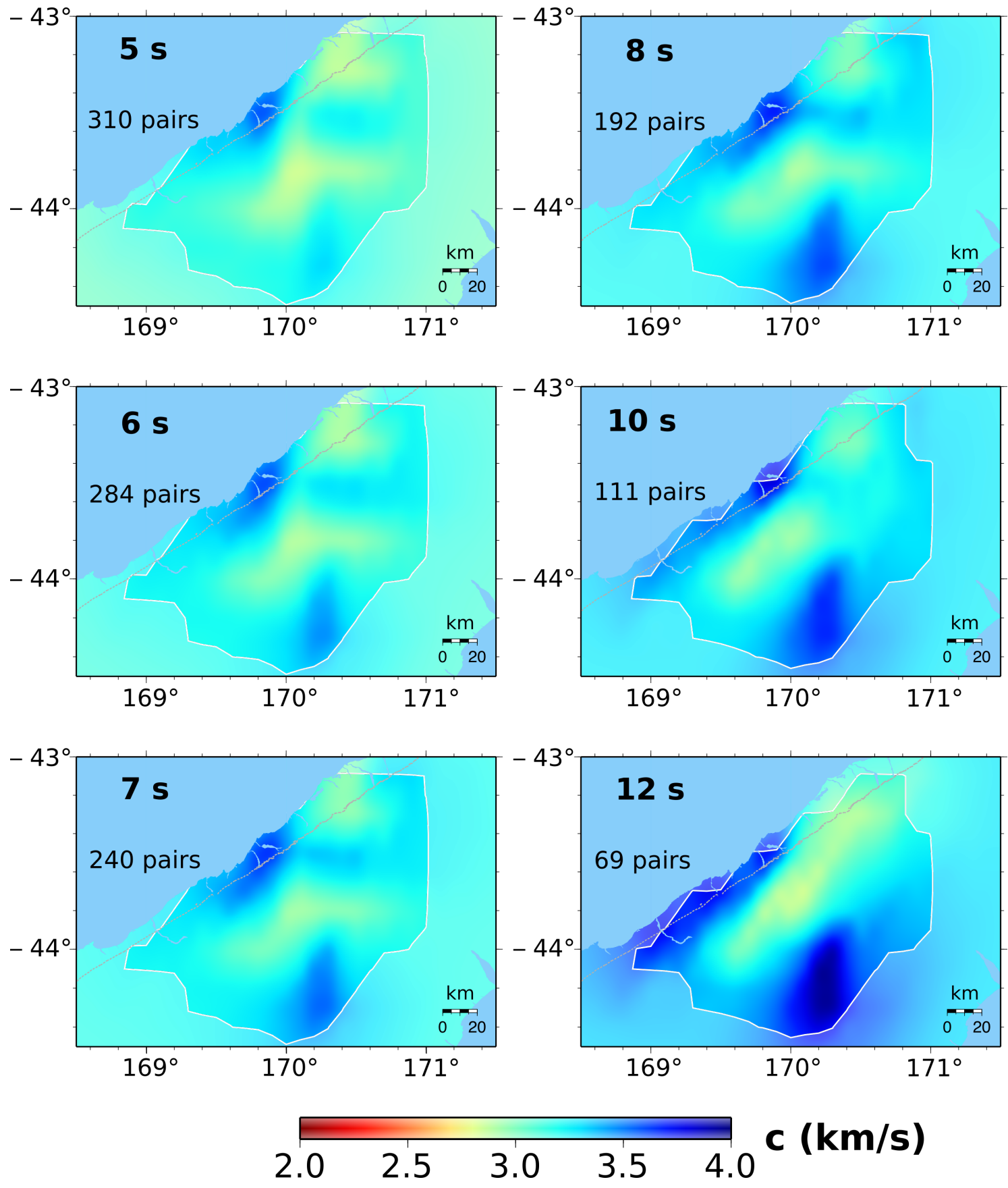

Figure 5.19: Love wave phase velocity maps for 5 to $12 \mathrm{~s}$ periods from TT crosscorrelations. Bold colours denote the model region where spatial resolution is $40 \mathrm{~km}$ or lower, with white contours outlining this region and colours outside the region masked. The surface expression of the Alpine Fault is indicated by the dashed grey line. 

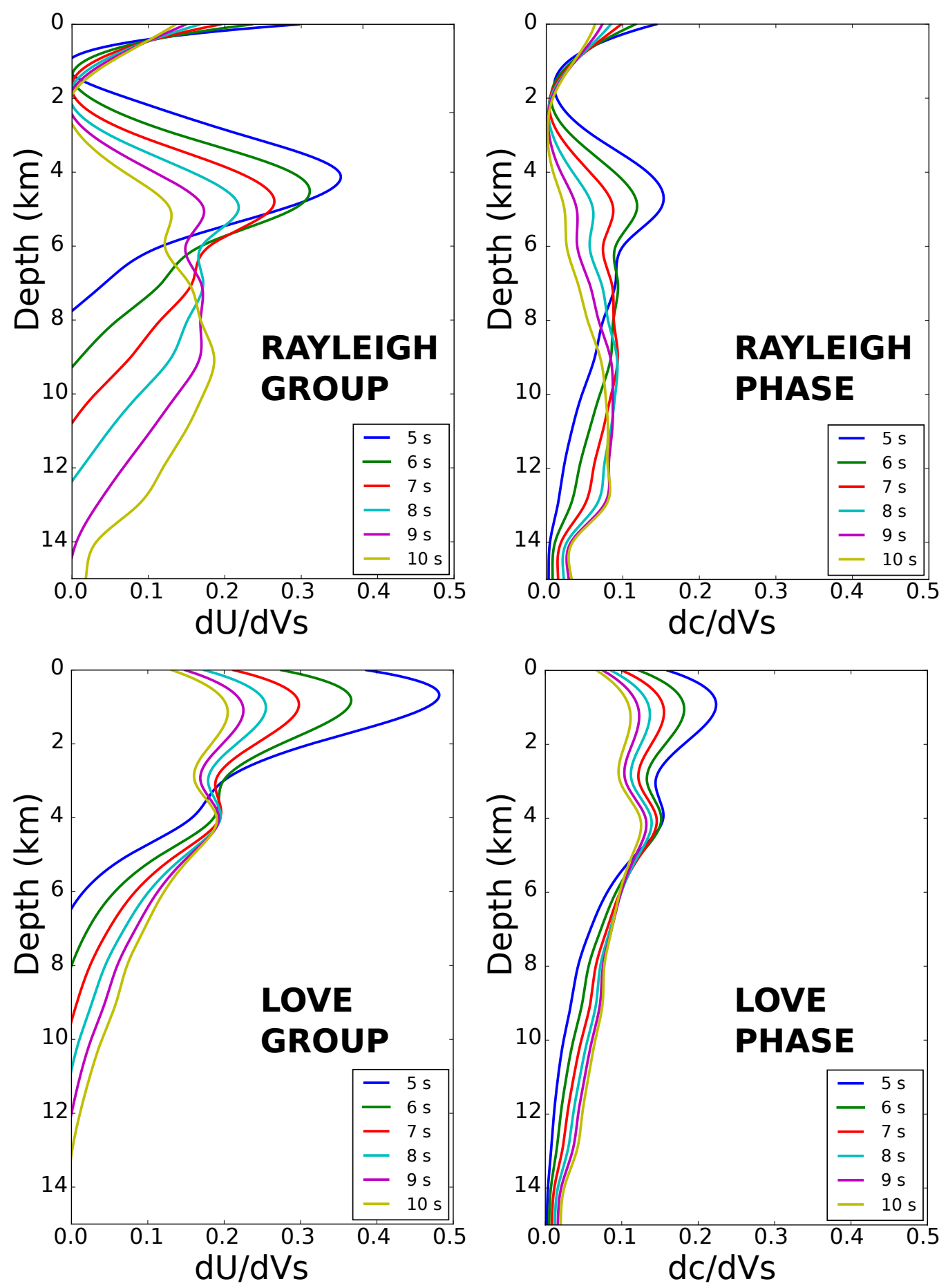

Figure 5.20: Sensitivity kernels for fundamental mode surface waves with respect to shear velocity in the central Alpine Fault region. An average regional velocity model from Eberhart-Phillips et al. (2010) was used to compute regional partial derivatives of surface waves for $5-10$ s periods. Group and phase velocity kernels are presented. 


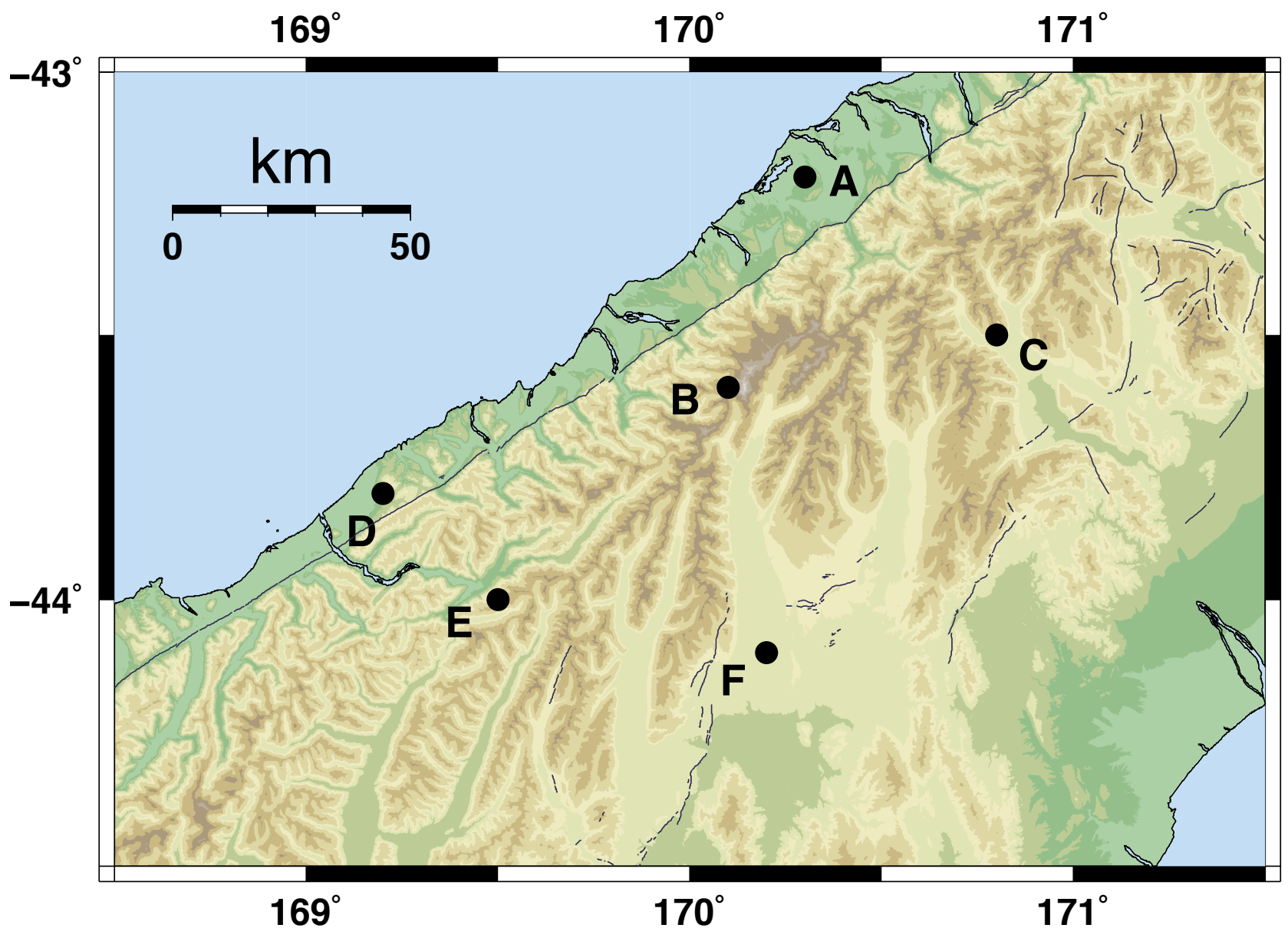

Figure 5.21: Locations of 1-D models A - F presented in Figures 5.22 and 5.23. 

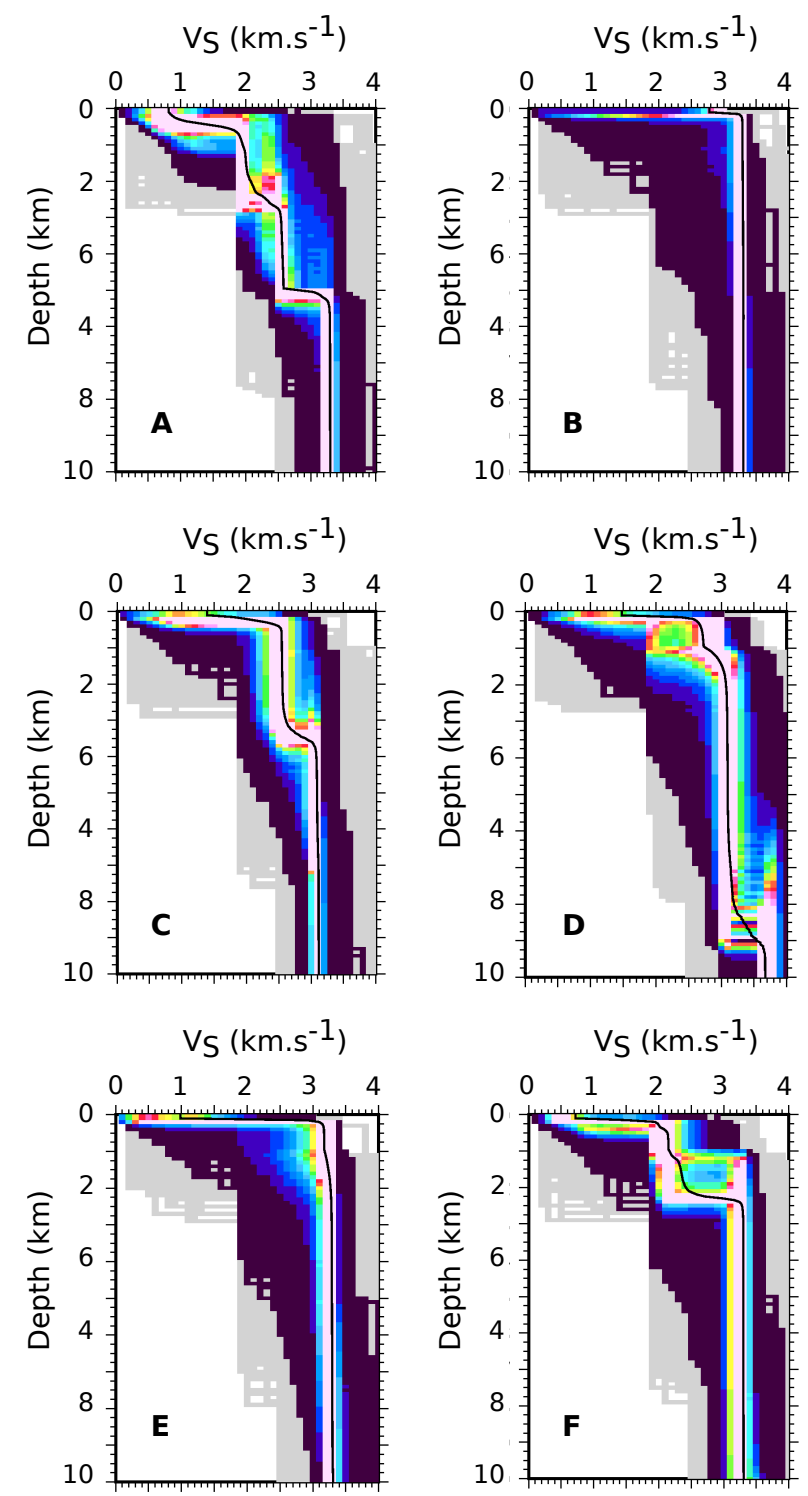

Figure 5.22: One-dimensional $\mathrm{V}_{S}$ models for the central Southern Alps from Rayleigh phase velocity measurements. For locations A - F (see Figure 5.21), inversions from Rayleigh phase velocity dispersion curves are presented. The preferred model, from all models with misfits below 0.05 , is shown as a black line, with coloured models increasing in warmth with decreasing misfit. Models in grey are those that were disregarded due to high model misfit values. 

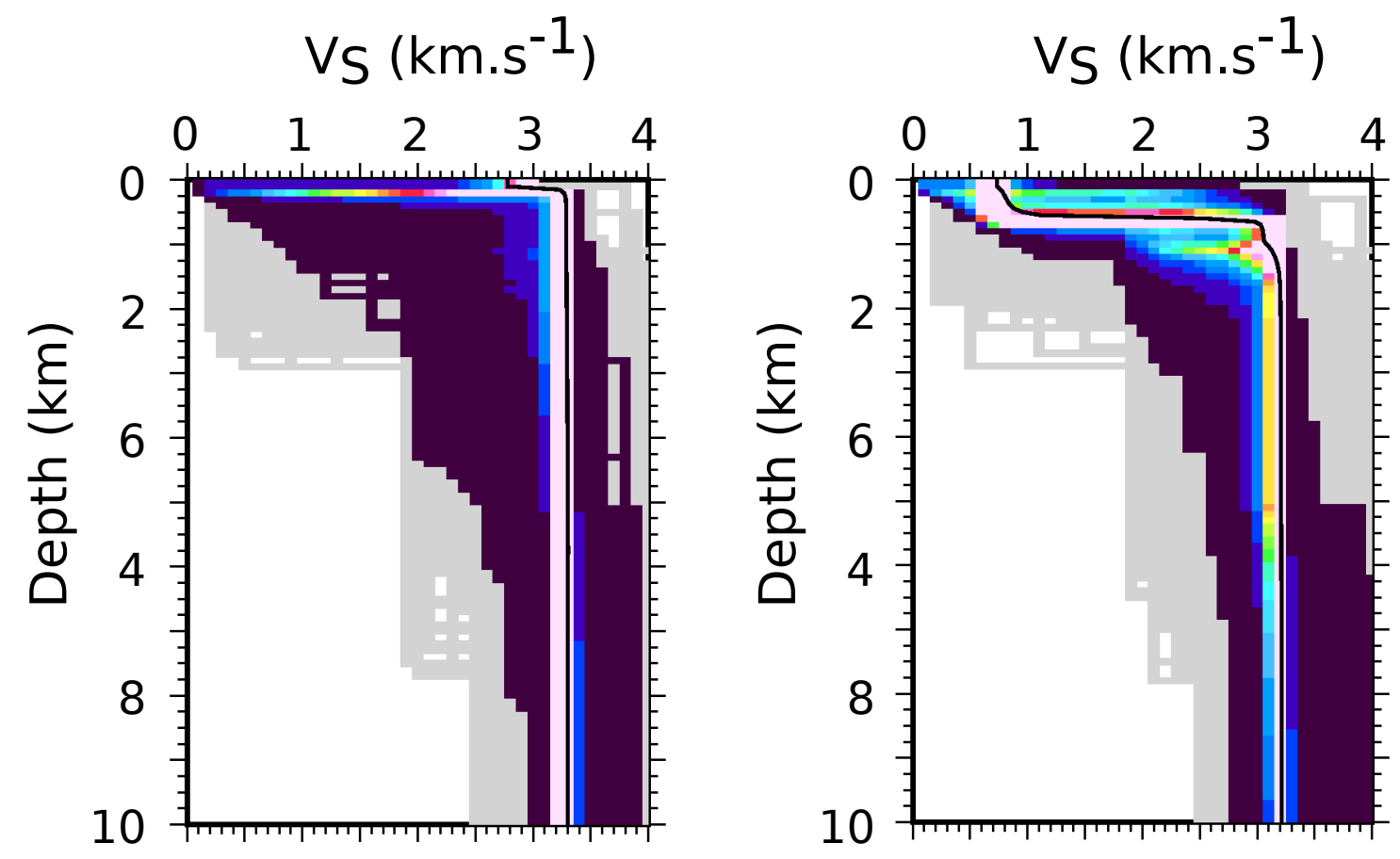

B: RAYLEIGH PHASE

\section{LOVE PHASE}
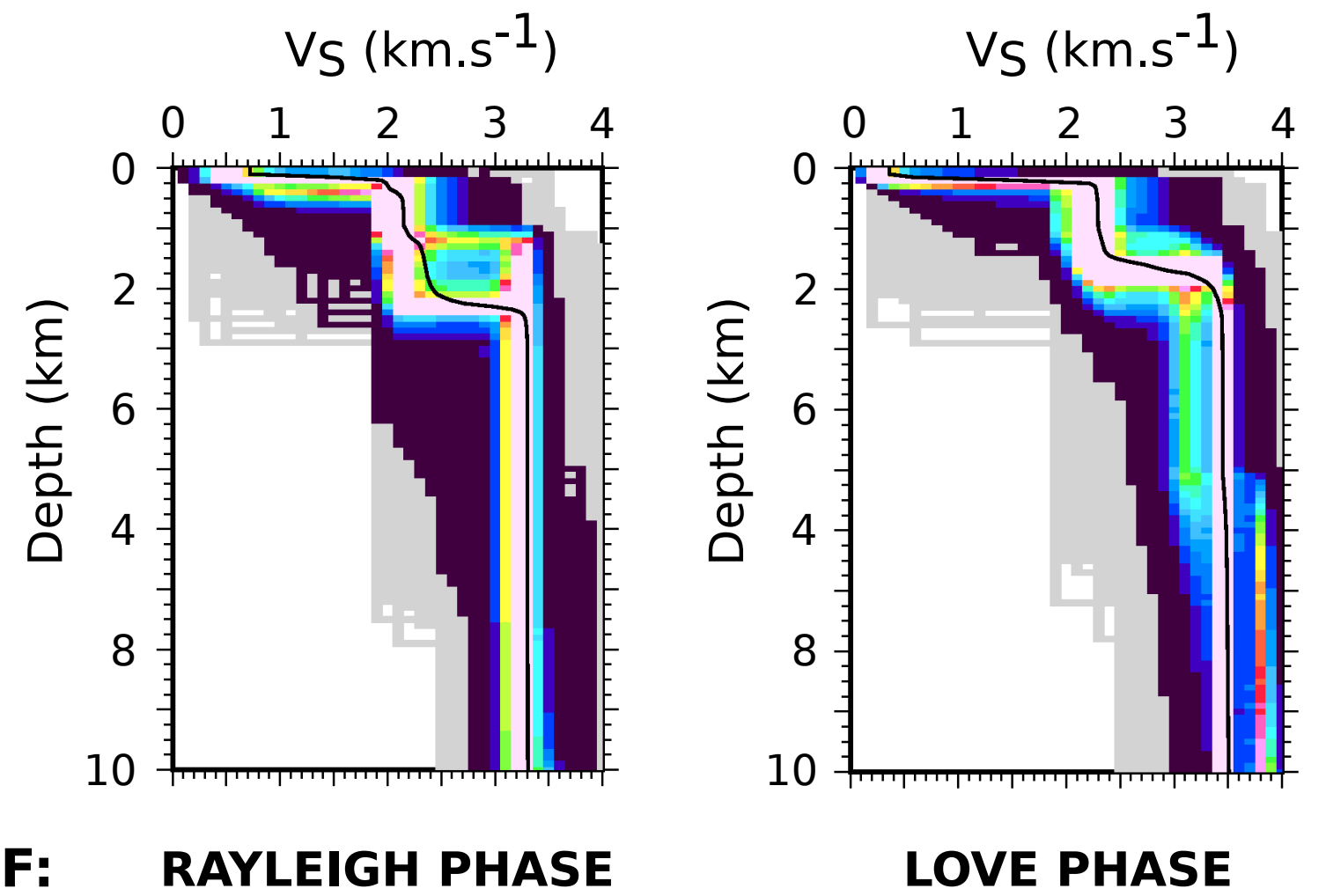

F: RAYLEIGH PHASE

Figure 5.23: One-dimensional $\mathrm{V}_{S}$ models for the central Southern Alps from Rayleigh phase velocity measurements. For locations B $(-43.6,170.1)$ and F (-44.1, 170.2), inversions from Rayleigh phase velocity dispersion curves are presented. The preferred model, from all models with misfits below 0.05, is shown as a black line, with coloured models increasing in warmth with decreasing misfit. Models in grey are those that were disregarded due to high model misfit values. 


\section{Discussion and Conclusions}

\subsection{Cross-correlation quality: comparisons between study regions}

To obtain reliable and well constrained $V_{S}$ models through ambient noise crosscorrelation, there are many procedures performed and so careful consideration of all input models, quality control and uncertainty criteria, and processing parameters is required. This Section gives a short dialogue into the findings of this study, with questions posed, and comparisons between regions will be made.

Firstly, what makes good ambient noise data? Ideally, recorded continuous seismic waveforms will be free from earthquakes and other impulsive seismic events (e.g. rockfalls, landslides), and monochromatic signals, (e.g. from urban machinery). Background noise should be of high amplitude and record a large frequency range. The Canterbury temporary networks were heavily contaminated with aftershocks of the Darfield earthquake, however the effect of these was effectively removed through the application of one-bit normalisation. The central Southern Alps experiences microseismicity and low frequency earthquakes, which have been well-documented in recent years (e.g Boese et al., 2012; Chamberlain et al., 2014). Again one-bit normalisation was extremely effective in removing these signals and is recommended for all study regions where background seismicity levels are high.

What quality control measures should be considered for cross-correlation functions, and how are appropriate thresholds determined? When considering signal-to-noise thresholds for surface-wave dispersion measurements, the calculated noise levels were comparable for both regions. This was somewhat unexpected, as the central Southern Alps study consisted of many more seismic stations, that had been well installed and established for many years prior to the considered time period. The data duration considered was significantly longer (one year compared to 4 months for Canterbury). The interstation paths covered a much larger range, and it would be expected that longer paths would experience higher attenuation and therefore 
have lower SNR's. This was not observed to the expected degree, and high quality dispersion measurements were still able to be obtained for long-path lengths. The SNR threshold was ultimately determined after observing the dispersion measurements for both regions and examining the spread of monthly dispersion curves. Correlations between temporary and permanent broadband stations gave different SNR's but both gave reliable dispersion measurements. So there is a great argument for including temporary deployments irregardless of data quality or deployment length.

Throughout this study it was observed that the greater control on tomography resolution was the wavelength constraint. For both regions a minimum wavelength of 2 was required, so that for velocity measurement computed in dispersion curves, 2 full wave cycles per station pair were required. This resulted in a rapid decrease in path coverage and spatial resolution with increasing period. However, even with a reduced path coverage at long periods, (as low as 30 interstation pairs for Canterbury over $9 \mathrm{~s}$ ), the resolution was still acceptable to proceed with tomography modelling and shear-wave inversions.

\subsection{Summary of key findings}

This work has obtained spatial and temporal velocity models from ambient seismic noise for the Canterbury Plains and the central Southern Alps regions of South Island, New Zealand. The following major observations and conclusions can be drawn from the work:

- Seismic velocity changes were measured for the four months following the 2010 $\mathrm{M}_{w}$ 7.1 Darfield earthquake, using the moving-window cross-spectral method. Increases of up to $0.15 \pm 0.04 \%$ were observed across the Canterbury region. This is comparable to velocity changes measured from ambient seismic noise following similar events. A depth analysis in conjunction with surface wave sensitivity kernels found that the temporal changes were isolated to the uppermost $5 \mathrm{~km}$ of the subsurface.

- Surface wave tomography and $\mathrm{V}_{S}$ models were obtained from surface wave dispersion measurements of cross-correlation functions throughout the Canterbury Plains. Models are in excellent agreement with previous velocity models and known geological features in the region. The uppermost subsurface layer is gives low velocities consistent with the sedimentary cover in the Canterbury Basin. The thickness of this shallow layer thins towards the foothills of the Southern Alps. The shear-wave velocities observed for the basement 
rock are consistent with average speeds for the known basement lithologies of greywackes and schists. High velocities are observed for basement rock in the southeastern extent of the modelled region. This is attributed to the volcanics of Banks Peninsula, although resolution is reduced here.

- One-dimensional shear velocity models located along the length of the Greendale Fault showed a surface damage layer, with $\mathrm{V}_{S}<1.0 \mathrm{~km} . \mathrm{s}^{-1}$ to depth of $500 \mathrm{~m}$. This especially low velocity layer was unique to the Greendale Fault, and confirms the interpretation of crack-healing obtained from the temporal surface-wave velocity monitoring.

- The presence of higher-mode Rayleigh waves investigated in Canterbury, as they have been shown to exist in high frequency cross-correlations (Savage et al., 2013). Synthetic Green's functions were able to approximate the crosscorrelation functions of (Savage et al., 2013). In our lower frequency band of interest, higher-mode Rayleigh waves were not generated. A thin shallow subsurface layer with markedly low velocities was required to produce the synthetic correlations, and this was subsequently shown to exist in 1-D shear models along the length of the Greendale Fault.

- Surface wave tomography and $\mathrm{V}_{S}$ models of the central Southern Alps were obtained. A high velocity region relating to the Southern Alps mountains was observed, with little-to-no sedimentary cover resolvable in the models. To the northwest of the Alpine Fault, in the hangingwall, a thin surface layer with low velocities was observed. To the southwest of the Southern Alps mountains, a thin, low velocity surface layer is also present, to thicknesses of $2 \mathrm{~km}$. Shear-wave inversions of Rayleigh and Love dispersion velocities were obtained separately and gave the best model convergence at different depths. In agreement with surface wave sensitivity kernels, Love waves resolve the uppermost $4-5 \mathrm{~km}$ better than Rayleigh waves, which are more sensitive to deeper structures.

- The dominant source of ambient seismic noise within South Island originates from Southern Ocean swells. Signal-to-noise ratios are consistently higher (up to an order of magnitude for some station pairs) for surface waves recorded travelling northwest. 


\subsection{Recommendations for future study}

Most avenues in future research lie with the SAMBA network and the Southern Alps.

- For this study one year of data from 2013 was processed for the central Southern Alps. A longer time period would increase signal-to-noise ratios and may allow higher quality dispersion measurements to be obtained.

- Seasonal trends in ambient seismic noise recorded in New Zealand have not been characterised. The use of the moving-window cross-spectral data for several years using the SAMBA network, or for New Zealand as a whole by using the GeoNet seismic network, may allow for variations due to atmospheric pressures or ocean current changes to be recorded.

- Temporal changes following the recent 2016-2017 Kaikoura earthquakes could be investigated. Using the moving-window cross-spectral method post-seismic relaxation may be observed. There were several seismic networks operational throughout the Marlborough region prior to the earthquakes, and so pre- and co- seismic velocity trends could be quantified.

- Using the SAMBA network and surrounding GeoNet stations, new constraints on anisotropy within the central Southern Alps could be attempted. Fry et al. (2014) mapped anisotropy throughout the Canterbury Plains using ambient seismic noise and the same method could be attempted on the SAMBA crosscorrelations. 
A Temporary Network Metadata 


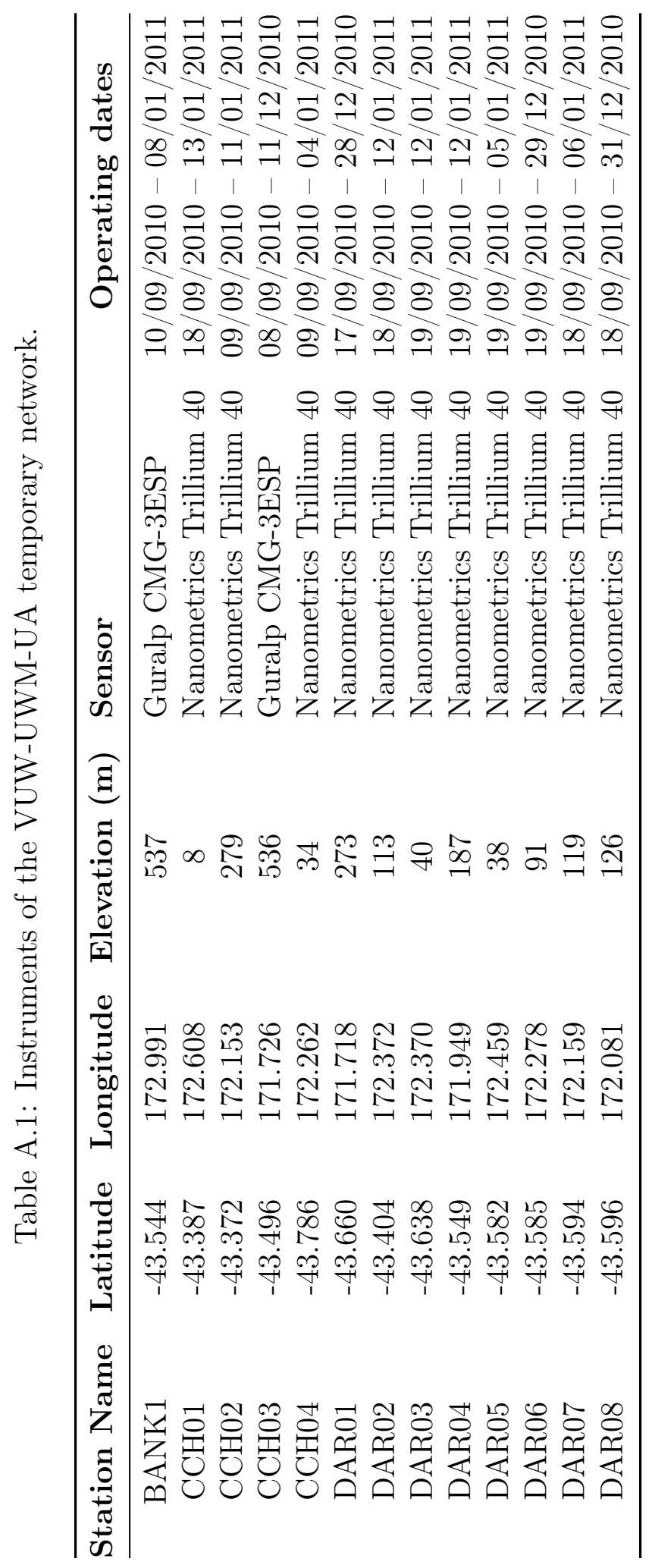




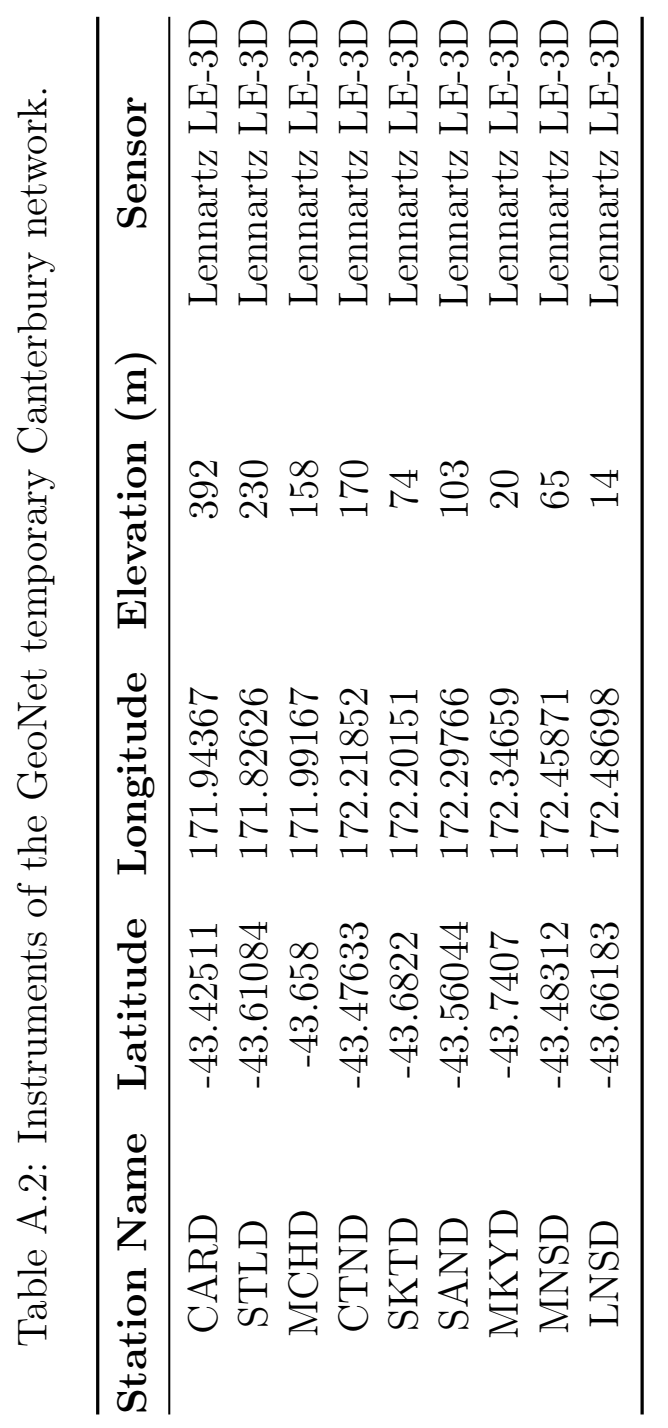




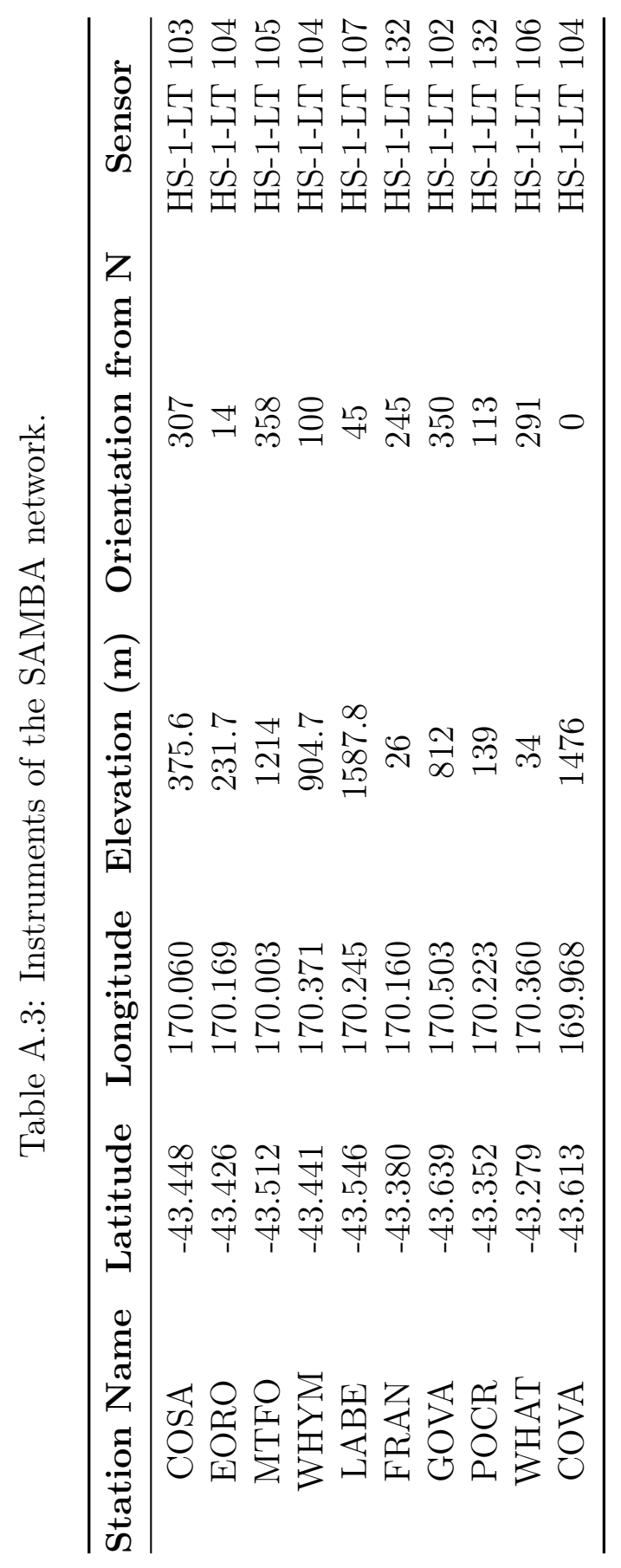


B Parameter and Starting Model Tables 
Table B.1: Average regional velocity model of Eberhart-Phillips et al. (2010) for the Canterbury Plains region

\begin{tabular}{cccc}
\hline Depth $(\mathrm{km})$ & $\mathrm{V}_{P}\left(\mathrm{~km} . \mathbf{s}^{-1}\right)$ & $\mathrm{V}_{S}\left(\mathrm{~km} . \mathbf{s}^{-1}\right)$ & Density $\left(\mathrm{kg} \cdot \mathbf{c m}^{3}\right)$ \\
\hline 1.0 & 3.850 & 2.292 & 2.6 \\
3.0 & 5.012 & 2.923 & 2.6 \\
8.0 & 5.884 & 3.445 & 2.7 \\
15.0 & 6.139 & 3.602 & 2.8 \\
23.0 & 6.696 & 3.904 & 3.0 \\
48.0 & 8.324 & 4.836 & 3.4 \\
65.0 & 8.396 & 4.854 & 3.4 \\
85.0 & 8.405 & 4.859 & 3.4 \\
105.0 & 8.436 & 4.875 & 3.4 \\
130.0 & 8.463 & 4.892 & 3.5 \\
155.0 & 8.420 & 4.865 & 3.4 \\
185.0 & 8.473 & 4.897 & 3.4 \\
225.0 & 8.560 & 4.950 & 3.5 \\
\hline
\end{tabular}

Table B.2: Two-layer velocity model of (Savage et al., 2013).

\begin{tabular}{cc}
\hline $\operatorname{Depth}(\mathrm{km})$ & $\mathrm{V}_{P}\left(\mathrm{~km} . \mathrm{s}^{-1}\right)$ \\
\hline 0 & 2.400 \\
1.5 & 5.400 \\
\hline
\end{tabular}

Table B.3: Average regional velocity model of Eberhart-Phillips et al. (2010) for the central Southern Alps region

\begin{tabular}{cccc}
\hline Depth $(\mathbf{k m})$ & $\mathbf{V}_{P}\left(\mathbf{k m . s ^ { - 1 }}\right)$ & $\mathbf{V}_{S}\left(\mathbf{k m} . \mathbf{s}^{-1}\right)$ & Density $\left.(\mathbf{k g . c m})^{3}\right)$ \\
\hline 1.0 & 3.850 & 2.292 & 2.7 \\
3.0 & 5.197 & 3.097 & 2.6 \\
8.0 & 5.978 & 3.556 & 2.7 \\
15.0 & 6.190 & 3.671 & 2.8 \\
23.0 & 6.462 & 3.806 & 2.9 \\
30.0 & 6.797 & 4.003 & 3.0 \\
38.0 & 7.555 & 4.437 & 3.2 \\
48.0 & 8.288 & 4.816 & 3.4 \\
65.0 & 8.442 & 4.879 & 3.4 \\
85.0 & 8.469 & 4.895 & 3.5 \\
105.0 & 8.451 & 4.892 & 3.4 \\
130.0 & 8.468 & 4.895 & 3.5 \\
155.0 & 8.430 & 4.870 & 3.4 \\
185.0 & 8.480 & 4.900 & 3.5 \\
225.0 & 8.560 & 4.950 & 3.5 \\
\hline
\end{tabular}


Table B.4: Canterbury model parameterisation

\begin{tabular}{cccc}
\hline $\mathrm{V}_{P}\left(\mathrm{~km} . \mathrm{s}^{-1}\right)$ & $\mathrm{V}_{S}\left(\mathrm{~km} . \mathrm{s}^{-1}\right)$ & Depth to layer base $(\mathrm{km})$ & Density $\left(\mathrm{kg} . \mathrm{cm}^{3}\right)$ \\
\hline $1.0-5.0$ & $1.0-2.5$ & $0.1-3.0$ & $2.0-4.0$ \\
$1.0-7.0$ & $1.9-3.5$ & $2.0-12.0$ & $2.0-4.0$ \\
$4.0-9.2$ & $2.5-4.0$ & - & $2.0-4.0$ \\
\hline
\end{tabular}

Table B.5: Alps model parameterisation

\begin{tabular}{cccc}
\hline $\mathrm{V}_{P}\left(\mathrm{~km} . \mathrm{s}^{-1}\right)$ & $\mathbf{V}_{S}\left(\mathbf{k m} . \mathbf{s}^{-1}\right)$ & Depth to layer base $(\mathrm{km})$ & Density $\left(\mathrm{kg} . \mathrm{cm}^{3}\right)$ \\
\hline $1.0-5.0$ & $1.0-3.0$ & $0.1-3.0$ & $2.0-4.0$ \\
$1.0-7.0$ & $1.9-4.0$ & $1.0-8.0$ & $2.0-4.0$ \\
$3.0-8.0$ & $2.5-4.5$ & $5.0-12.0$ & $2.0-4.0$ \\
$4.0-9.2$ & $3.0-5.0$ & - & $2.0-4.0$ \\
\hline
\end{tabular}




\section{Bibliography}

Aki, K. (1957). Space and time spectra of stationary stochastic waves, with special reference to microtremors. Bulletin of the Earthquake Research Institute, 35:415457.

Aki, K. and Richards, P. G. (2002). Quantitative seismology. University Science Books, 2nd edition.

Aster, R. C. and Shearer, P. M. (1991). High-frequency borehole seismograms recorded in the San Jacinto fault zone, southern California. Part 1. Polarizations. Bulletin of the Seismological Society of America, 81(4):1057-1080.

Ball, J. S., Sheehan, A. F., Stachnik, J. C., Lin, F.-C., Yeck, W. L., and Collins, J. A. (2016). Lithospheric shear velocity structure of South Island, New Zealand, from amphibious Rayleigh wave tomography. Journal of Geophysical Research: Solid Earth, 121(5):3686-3702.

Bannister, S. and Gledhill, K. (2012). Evolution of the 2010-2012 Canterbury earthquake sequence. New Zealand Journal of Geology and Geophysics, 55(3):295-304.

Barmin, M., Ritzwoller, M., and Levshin, A. (2001). A Fast and Reliable Method for Surface Wave Tomography. Pure appl. geophys, 158(1351):1375.

Beavan, J., Motagh, M., Fielding, E. J., Donnelly, N., and Collett, D. (2012). Fault slip models of the 2010-2011 Canterbury, New Zealand, earthquakes from geodetic data and observations of postseismic ground deformation. New Zealand Journal of Geology and Geophysics, 55(3):207-221.

Behr, Y. (2011). Imaging New Zealand's crustal structure using ambient seismic noise recordings from permanent and temporary instruments. PhD thesis, Victoria University of Wellington.

Behr, Y., Townend, J., Bannister, S., and Savage, M. (2010). Shear velocity structure of the Northland Peninsula, New Zealand, inferred from ambient noise correlations. Journal of Geophysical Research: Solid Earth (1978-2012), 115(B5).

Behr, Y., Townend, J., Bannister, S., and Savage, M. (2011). Crustal shear wave 
tomography of the Taupo Volcanic Zone, New Zealand, via ambient noise correlation between multiple three-component networks. Geochemistry, Geophysics, Geosystems, 12(3).

Behr, Y., Townend, J., Bowen, M., Carter, L., Gorman, R., Brooks, L., and Bannister, S. (2013). Source directionality of ambient seismic noise inferred from three-component beamforming. Journal of Geophysical Research: Solid Earth, 118(1):240-248.

Bensen, G., Ritzwoller, M., Barmin, M., Levshin, A., Lin, F., Moschetti, M., Shapiro, N., and Yang, Y. (2007). Processing seismic ambient noise data to obtain reliable broad-band surface wave dispersion measurements. Geophysical Journal International, 169(3):1239-1260.

Berryman, K. R., Cochran, U. A., Clark, K. J., Biasi, G. P., Langridge, R. M., and Villamor, P. (2012). Major earthquakes occur regularly on an isolated plate boundary fault. Science, 336(6089):1690-1693.

Boese, C., Townend, J., Smith, E., and Stern, T. (2012). Microseismicity and stress in the vicinity of the Alpine Fault, central Southern Alps, New Zealand. Journal of Geophysical Research: Solid Earth, 117(B2).

Bonnefoy-Claudet, S., Cotton, F., and Bard, P.-Y. (2006). The nature of noise wavefield and its applications for site effects studies: a literature review. EarthScience Reviews, 79(3):205-227.

Bourguignon, S., Bannister, S., Henderson, C. M., Townend, J., and Zhang, H. (2015). Structural heterogeneity of the midcrust adjacent to the central Alpine Fault, New Zealand: Inferences from seismic tomography and seismicity between Harihari and Ross. Geochemistry, Geophysics, Geosystems, 16(4):1017-1043.

Bracewell, R. N. (1978). The Fourier Transform and Its Application. McGraw-Hill, New York.

Brenguier, F., Campillo, M., Hadziioannou, C., Shapiro, N., Nadeau, R. M., and Larose, E. (2008). Postseismic relaxation along the San Andreas fault at Parkfield from continuous seismological observations. Science, 321(5895):1478-1481.

Brenguier, F., Shapiro, N. M., Campillo, M., Nercessian, A., and Ferrazzini, V. (2007). 3-D surface wave tomography of the Piton de la Fournaise volcano using seismic noise correlations. Geophysical research letters, 34(2).

Brooks, L. A., Townend, J., Gerstoft, P., Bannister, S., and Carter, L. (2009). Fundamental and higher-mode Rayleigh wave characteristics of ambient seismic noise in New Zealand. Geophysical Research Letters, 36(23). 
Browne, G., Field, B., Barrell, D., Jongens, R., Bassett, K., and Wood, R. (2012). The geological setting of the Darfield and Christchurch earthquakes. New Zealand Journal of Geology and Geophysics, 55(3):193-197.

Campillo, M. and Paul, A. (2003). Long-range correlations in the diffuse seismic coda. Science, 299(5606):547-549.

Chamberlain, C. J., Boese, C. M., and Townend, J. (2017). Cross-correlation-based detection and characterisation of microseismicity adjacent to the locked, lateinterseismic Alpine Fault, South Westland, New Zealand. Earth and Planetary Science Letters, 457:63-72.

Chamberlain, C. J., Shelly, D. R., Townend, J., and Stern, T. A. (2014). Lowfrequency earthquakes reveal punctuated slow slip on the deep extent of the Alpine Fault, New Zealand. Geochemistry, Geophysics, Geosystems, 15(7):2984-2999.

Chiu, H.-C., Huang, H.-C., Leu, C.-L., and Ni, S.-D. (1994). Application of polarization analysis in correcting the orientation error of a downhole seismometer. Earthquake engineering and structural dynamics, 23(10):1069-1078.

Claerbout, J. F. (1968). Synthesis of a layered medium from its acoustic transmission response. Geophysics, 33(2):264-269.

Clarke, D., Zaccarelli, L., Shapiro, N., and Brenguier, F. (2011). Assessment of resolution and accuracy of the Moving Window Cross Spectral technique for monitoring crustal temporal variations using ambient seismic noise. Geophysical Journal International, 186(2):867-882.

Cox, S., Rutter, H., Sims, A., Manga, M., Weir, J., Ezzy, T., White, P., Horton, T., and Scott, D. (2012). Hydrological effects of the MW 7.1 Darfield (Canterbury) earthquake, 4 September 2010, New Zealand. New Zealand Journal of Geology and Geophysics, 55(3):231-247.

Cox, S. C. and Sutherland, R. (2007). Regional geological framework of South Island, New Zealand, and its significance for understanding the active plate boundary. $A$ continental plate boundary: Tectonics at South Island, New Zealand, pages 19-46.

Davy, B., Hoernle, K., and Werner, R. (2008). Hikurangi Plateau: Crustal structure, rifted formation, and Gondwana subduction history. Geochemistry, Geophysics, Geosystems, 9(7).

DeMets, C., Gordon, R. G., Argus, D. F., and Stein, S. (1994). Effect of recent revisions to the geomagnetic reversal time scale on estimates of current plate motions. Geophysical Research Letters, 21(20):2191-2194. 
Duvall, T., Jeffferies, S., Harvey, J., and Pomerantz, M. (1993). Time-distance helioseismology. Nature, 362(6419):430-432.

Dziewonski, A., Bloch, S., and Landisman, M. (1969). A technique for the analysis of transient seismic signals. Bulletin of the seismological Society of America, $59(1): 427-444$.

Eberhart-Phillips, D. and Bannister, S. (2002). Three-dimensional crustal structure in the Southern Alps region of New Zealand from inversion of local earthquake and active source data. Journal of Geophysical Research: Solid Earth (1978-2012), 107(B10):ESE-15.

Eberhart-Phillips, D., Reyners, M., Bannister, S., Chadwick, M., and Ellis, S. (2010). Establishing a versatile 3-D seismic velocity model for New Zealand. Seismological Research Letters, 81(6):992-1000.

Feenstra, J., Thurber, C., Townend, J., Roecker, S., Bannister, S., Boese, C., Lord, N., Bourguignon, S., and Eberhart-Phillips, D. (2016). Microseismicity and $\mathrm{P}$-wave tomography of the central Alpine Fault, New Zealand. New Zealand Journal of Geology and Geophysics, 59(4):483-495.

Forsyth, P., Barrell, D., and Jongens, R. (2008). Geology of the Christchurch Area, Institute of Geological and Nuclear Sciences 1: 250000 geological map 16. Lower Hutt, New Zealand.

Friedrich, A., Krüger, F., and Klinge, K. (1998). Ocean-generated microseismic noise located with the Gräfenberg array. Journal of Seismology, 2(1):47-64.

Fry, B., Davey, F., Eberhart-Phillips, D., and Lebedev, S. (2014). Depth variable crustal anisotropy, patterns of crustal weakness, and destructive earthquakes in Canterbury, New Zealand. Earth and Planetary Science Letters, 392:50-57.

Fry, B. and Gerstenberger, M. (2011). Large apparent stresses from the Canterbury earthquakes of 2010 and 2011. Seismological Research Letters, 82(6):833-838.

GeoNet (2016). Continuous Waveform Buffer. http://info.geonet.org.nz/ display/appdata/Continuous+Waveform+Buffer. Last accessed 7 October 2016 .

Ghisetti, F. and Sibson, R. (2012). Compressional reactivation of E-W inherited normal faults in the area of the 2010-2011 Canterbury earthquake sequence. New Zealand Journal of Geology and Geophysics, 55(3):177-184.

Giles, P., Duvall, T., Scherrer, P., and Bogart, R. (1997). A subsurface flow of material from the Sun's equator to its poles. Nature, 390(6655):52-54. 
Gledhill, K., Ristau, J., Reyners, M., Fry, B., and Holden, C. (2011). The Darfield (Canterbury, New Zealand) Mw 7.1 earthquake of September 2010: A preliminary seismological report. Seismological Research Letters, 82(3):378-386.

Godfrey, H. J., Fry, B., and Savage, M. K. (2017). Shear-wave velocity structure of the Tongariro Volcanic Centre, New Zealand: Fast Rayleigh and slow Love waves indicate strong shallow anisotropy. Journal of Volcanology and Geothermal Research.

Goldstein, P. and Snoke, A. (2005). SAC availability for the IRIS community. Incorporated Institutions for Seismology Data Management Center Electronic Newsletter, $7(1)$.

Gubbins, D. (2004). Time series analysis and inverse theory for geophysicists. Cambridge University Press, Cambridge, UK.

Gulley, A. K., Ward, N. F. D., Cox, S. C., and Kaipio, J. P. (2013). Groundwater responses to the recent Canterbury earthquakes: a comparison. Journal of Hydrology, 504:171-181.

Gutenberg, B. (1958). Microseisms. Advances in Geophysics, 5:53-92.

Harmon, N., Forsyth, D., and Webb, S. (2007). Using ambient seismic noise to determine short-period phase velocities and shallow shear velocities in young oceanic lithosphere. Bulletin of the Seismological Society of America, 97(6):2009-2023.

Hasselmann, K. (1963). A statistical analysis of the generation of microseisms. Reviews of Geophysics, 1(2):177-210.

Havskov, J. and Alguacil, J. (2004). Instrumentation in Earthquake Seismology Springer. Dordrecht, The Netherlands.

Heckels, R. E. G., Savage, M., and Townend, J. (submitted 2017). Postseismic velocity variation following the $2010 \mathrm{M}_{w} 7.1$ Darfield earthquake, New Zealand, revealed by ambient seismic noise analyses. Geophysical Journal International.

Herrmann, R. and Ammon, C. (2004). Surface waves, receiver functions and crustal structure. Computer Programs in Seismology, Version, 3:30.

Hillers, G., Ben-Zion, Y., Campillo, M., and Zigone, D. (2015). Seasonal variations of seismic velocities in the San Jacinto fault area observed with ambient seismic noise. Geophysical Journal International, 202(2):920-932.

Hobiger, M., Wegler, U., Shiomi, K., and Nakahara, H. (2012). Coseismic and postseismic elastic wave velocity variations caused by the 2008 Iwate-Miyagi Nairiku earthquake, Japan. Journal of Geophysical Research: Solid Earth, 117(B9). 
Holt, R., Savage, M., Townend, J., Syracuse, E., and Thurber, C. (2013). Crustal stress and fault strength in the Canterbury Plains, New Zealand. Earth and Planetary Science Letters, 383:173-181.

Kaiser, A., Holden, C., Beavan, J., Beetham, D., Benites, R., Celentano, A., Collett, D., Cousins, J., Cubrinovski, M., Dellow, G., et al. (2012). The Mw 6.2 Christchurch earthquake of February 2011: preliminary report. New Zealand journal of geology and geophysics, 55(1):67-90.

Lecocq, T., Caudron, C., and Brenguier, F. (2014). MSNoise, a Python Package for Monitoring Seismic Velocity Changes Using Ambient Seismic Noise. Seismological Research Letters, 85(3):715-726.

Levshin, A., Pisarenko, V., and Pogrebinsky, G. (1972). On a frequency-time analysis of oscillations. Annals of Geophysics, 28(2):211-218.

Levshin, A., Ratnikova, L., and Berger, J. (1992). Peculiarities of surface-wave propagation across central Eurasia. Bulletin of the Seismological Society of America, 82(6):2464-2493.

Levshin, A. and Ritzwoller, M. (2001). Automated detection, extraction, and measurement of regional surface waves. In Monitoring the Comprehensive NuclearTest-Ban Treaty: Surface Waves, pages 1531-1545. Springer, New York.

Levshin, A., Yanovskaya, T., Lander, A., Bukchin, B., Barmin, M., Ratnikova, L., and Its, E. (1989). Seismic surface waves in a laterally inhomogeneous Earth. Modern Approaches in Geophysics, 9:131-169.

Lin, F.-C., Moschetti, M. P., and Ritzwoller, M. H. (2008). Surface wave tomography of the western United States from ambient seismic noise: Rayleigh and Love wave phase velocity maps. Geophysical Journal International, 173(1):281-298.

Lin, F.-C., Ritzwoller, M. H., Townend, J., Bannister, S., and Savage, M. K. (2007). Ambient noise Rayleigh wave tomography of New Zealand. Geophysical Journal International, 170(2):649-666.

Lin, F.-C., Schmandt, B., and Tsai, V. C. (2012). Joint inversion of Rayleigh wave phase velocity and ellipticity using USArray: Constraining velocity and density structure in the upper crust. Geophysical Research Letters, 39(12).

Liu, Z., Huang, J., Peng, Z., and Su, J. (2014). Seismic velocity changes in the epicentral region of the 2008 Wenchuan earthquake measured from three-component ambient noise correlation techniques. Geophysical Research Letters, 41(1):37-42.

Lobkis, O. I. and Weaver, R. L. (2001). On the emergence of the Green's function 
in the correlations of a diffuse field. The Journal of the Acoustical Society of America, 110(6):3011-3017.

Longuet-Higgins, M. S. (1950). A theory of the origin of microseisms. Philosophical Transactions of the Royal Society of London A: Mathematical, Physical and Engineering Sciences, 243(857):1-35.

Louie, J. N. (2001). Faster, better: shear-wave velocity to 100 meters depth from refraction microtremor arrays. Bulletin of the Seismological Society of America, 91(2):347-364.

Love, A. E. H. (1911). Some Problems of Geodynamics. Cambridge University Press, Cambridge, UK.

Magistrale, H., Day, S., Clayton, R. W., and Graves, R. (2000). The SCEC southern California reference three-dimensional seismic velocity model version 2. Bulletin of the Seismological Society of America, 90(6B):S65-S76.

Mainsant, G., Larose, E., Brönnimann, C., Jongmans, D., Michoud, C., and Jaboyedoff, M. (2012). Ambient seismic noise monitoring of a clay landslide: Toward failure prediction. Journal of Geophysical Research: Earth Surface, 117(F1).

Minato, S., Tsuji, T., Ohmi, S., and Matsuoka, T. (2012). Monitoring seismic velocity change caused by the 2011 Tohoku-oki earthquake using ambient noise records. Geophysical Research Letters, 39(9).

Mordret, A., Landès, M., Shapiro, N., Singh, S., Roux, P., and Barkved, O. (2013). Near-surface study at the Valhall oil field from ambient noise surface wave tomography. Geophysical Journal International, page ggt061.

Mortimer, N. (2004). New Zealand's geological foundations. Gondwana Research, $7(1): 261-272$.

Mortimer, N. and Campbell, H. (2014). Zealandia: our continent revealed. Penguin Books, New Zealand.

Norris, R. J. and Cooper, A. F. (2001). Late Quaternary slip rates and slip partitioning on the Alpine Fault, New Zealand. Journal of Structural Geology, 23(2):507520.

Okaya, D., Stern, T., Davey, F., Henrys, S., and Cox, S. (2007). Continent-Continent Collision at the Pacific/Indo-Australian Plate Boundary: Background, Motivation, and Principal Results. A Continental Plate Boundary: Tectonics at South Island, New Zealand, pages 1-18.

Olsen, K., Day, S., Minster, J., Cui, Y., Chourasia, A., Faerman, M., Moore, R., 
Maechling, P., and Jordan, T. (2006). Strong shaking in Los Angeles expected from southern San Andreas earthquake. Geophysical Research Letters, 33(7).

Petersen, T., Gledhill, K., Chadwick, M., Gale, N. H., and Ristau, J. (2011). The New Zealand national seismograph network. Seismological Research Letters, 82(1):9-20.

Poli, P., Pedersen, H., Campillo, M., Group, P. W., et al. (2012). Noise directivity and group velocity tomography in a region with small velocity contrasts: the northern Baltic shield. Geophysical Journal International, 192(1):413-424.

Poupinet, G., Ellsworth, W., and Frechet, J. (1984). Monitoring velocity variations in the crust using earthquake doublets: An application to the Calaveras Fault, California. Journal of Geophysical Research: Solid Earth, 89(B7):5719-5731.

Project, G. (2017). Dinver Software. http://www.geopsy.org/. Last accessed 7 April 2017.

Quigley, M., Van Dissen, R., Litchfield, N., Villamor, P., Duffy, B., Barrell, D., Furlong, K., Stahl, T., Bilderback, E., and Noble, D. (2012). Surface rupture during the $2010 \mathrm{Mw} 7.1$ Darfield (Canterbury) earthquake: Implications for fault rupture dynamics and seismic-hazard analysis. Geology, 40(1):55-58.

Ratdomopurbo, A. and Poupinet, G. (1995). Monitoring a temporal change of seismic velocity in a volcano: Application to the 1992 eruption of Mt. Merapi (Indonesia). Geophysical Research Letters, 22(7):775-778.

Rayleigh, J. W. S. (1885). On waves propagated along the plane surface of an elastic solid. Proceedings of the London Mathematical Society, 17:4-11.

Reyners, M., Eberhart-Phillips, D., and Martin, S. (2014). Prolonged Canterbury earthquake sequence linked to widespread weakening of strong crust. Nature Geoscience, $7(1): 34-37$.

Rickett, J. and Claerbout, J. (2000). Calculation of the Sun's acoustic impulse response by multi-dimensional spectral factorization. Solar Physics, 192(1-2):203210.

Ritzwoller, M. H. and Levshin, A. L. (1998). Eurasian surface wave tomography: Group velocities. Journal of Geophysical Research: Solid Earth, 103(B3):48394878.

Rivet, D., Campillo, M., Radiguet, M., Zigone, D., Cruz-Atienza, V., Shapiro, N. M., Kostoglodov, V., Cotte, N., Cougoulat, G., Walpersdorf, A., et al. (2014). Seismic velocity changes, strain rate and non-volcanic tremors during the 2009-2010 slow 
slip event in Guerrero, Mexico. Geophysical Journal International, 196(1):447460 .

Roueff, A., Roux, P., and Réfrégier, P. (2009). Wave separation in ambient seismic noise using intrinsic coherence and polarization filtering. Signal Processing, $89(4): 410-421$.

Roux, P. (2009). Passive seismic imaging with directive ambient noise: application to surface waves and the San Andreas Fault in Parkfield, CA. Geophysical Journal International, 179(1):367-373.

Sabra, K. G., Gerstoft, P., Roux, P., Kuperman, W., and Fehler, M. C. (2005). Extracting time-domain Green's function estimates from ambient seismic noise. Geophysical Research Letters, 32(3).

Sambridge, M. (1999a). Geophysical inversion with a neighbourhood algorithm-I. Searching a parameter space. Geophysical Journal International, 138(2):479-494.

Sambridge, M. (1999b). Geophysical inversion with a neighbourhood algorithm-II. Appraising the ensemble. Geophysical Journal International, 138(3):727-746.

Sánchez-Sesma, F. J. and Campillo, M. (2006). Retrieval of the Green's function from cross correlation: the canonical elastic problem. Bulletin of the Seismological Society of America, 96(3):1182-1191.

Savage, M. K., Lin, F.-C., and Townend, J. (2013). Ambient noise cross-correlation observations of fundamental and higher-mode Rayleigh wave propagation governed by basement resonance. Geophysical Research Letters, 40(14):3556-3561.

Scherbaum, F. (2013). Of poles and zeros: Fundamentals of digital seismology, volume 15. Springer Science and Business Media, New York, 3rd edition.

Sens-Schönfelder, C. and Larose, E. (2008). Temporal changes in the lunar soil from correlation of diffuse vibrations. Physical Review E, 78(4):045601.

Sens-Schönfelder, C. and Wegler, U. (2006). Passive image interferometry and seasonal variations of seismic velocities at Merapi Volcano, Indonesia. Geophysical research letters, 33(21).

Sewell, R., Weaver, S., and Reay, M. (1992). Geology of Banks Peninsula. Institute of Geological and Nuclear Sciences Limited, New Zealand.

Shapiro, N. M. and Campillo, M. (2004). Emergence of broadband Rayleigh waves from correlations of the ambient seismic noise. Geophysical Research Letters, $31(7)$. 
Shapiro, N. M., Campillo, M., Stehly, L., and Ritzwoller, M. H. (2005). Highresolution surface-wave tomography from ambient seismic noise. Science, 307(5715):1615-1618.

Sibson, R., Ghisetti, F., and Ristau, J. (2011). Stress control of an evolving strikeslip fault system during the 2010-2011 Canterbury, New Zealand, earthquake sequence. Seismological Research Letters, 82(6):824-832.

Snieder, R. (2004). Extracting the Green's function from the correlation of coda waves: A derivation based on stationary phase. Physical Review E, 69(4):046610.

Snieder, R., Grêt, A., Douma, H., and Scales, J. (2002). Coda wave interferometry for estimating nonlinear behavior in seismic velocity. Science, 295(5563):22532255 .

Stehly, L., Fry, B., Campillo, M., Shapiro, N., Guilbert, J., Boschi, L., and Giardini, D. (2009). Tomography of the Alpine region from observations of seismic ambient noise. Geophysical Journal International, 178(1):338-350.

Stein, S. and Wysession, M. (2003). An Introduction to Seismology, Earthquakes and Earth Structure. Blackwell Publishing, Oxford, UK.

Stern, T., Okaya, D., Kleffmann, S., Scherwath, M., Henrys, S., and Davey, F. (2007). Geophysical exploration and dynamics of the Alpine Fault zone. A Continental Plate Boundary: Tectonics at South Island, New Zealand, pages 207-233.

Sutherland, R., Eberhart-Phillips, D., Harris, R., Stern, T., Beavan, J., Ellis, S., Henrys, S., Cox, S., Norris, R., Berryman, K., et al. (2007). Do great earthquakes occur on the alpine fault in central south island, new zealand? A Continental Plate Boundary: Tectonics at South Island, New Zealand, pages 235-251.

Sutherland, R., Toy, V., et al. (2009). Deep Fault Drilling Project - Alpine Fault, New Zealand. Scientific drilling, 8:75-82.

Syracuse, E., Holt, R., Savage, M., Johnson, J., Thurber, C., Unglert, K., Allan, K., Karaliyadda, S., and Henderson, M. (2012). Temporal and spatial evolution of hypocentres and anisotropy from the Darfield aftershock sequence: implications for fault geometry and age. New Zealand Journal of Geology and Geophysics, $55(3): 287-293$.

Syracuse, E., Thurber, C., Rawles, C., Savage, M., and Bannister, S. (2013). Highresolution relocation of aftershocks of the Mw 7.1 Darfield, New Zealand, earthquake and implications for fault activity. Journal of Geophysical Research: Solid Earth, 118(8):4184-4195. 
Thurber, C., Roecker, S., Townend, J., Stern, T., and Lord, N. (2012). WIsconsin, New Zealand, And Rensselaer Deployment. doi:10.7914/SN/ZT_2012. International Federation of Digital Seismograph Networks.

Thurber, C. H., Roecker, S. W., Feenstra, J., Lord, N., O’Brien, G., Pesicek, J. D., Bannister, S. C., and Townend, J. (2012). Seismic site characterization for the Deep Fault Drilling Project (DFDP), Alpine Fault, New Zealand: Preliminary results from the WIZARD array. AGU Fall Meeting Abstracts.

Townend, J., Sherburn, S., Arnold, R., Boese, C., and Woods, L. (2012). Threedimensional variations in present-day tectonic stress along the Australia-Pacific plate boundary in New Zealand. Earth and Planetary Science Letters, 353:47-59.

Tsai, V. C. (2010). The relationship between noise correlation and the Green's function in the presence of degeneracy and the absence of equipartition. Geophysical Journal International, 182(3):1509-1514.

Van Avendonk, H. J., Holbrook, W. S., Okaya, D., Austin, J. K., Davey, F., and Stern, T. (2004). Continental crust under compression: A seismic refraction study of South Island Geophysical Transect I, South Island, New Zealand. Journal of Geophysical Research: Solid Earth, 109(B6).

Walcott, R. (1998). Modes of oblique compression: Late Cenozoic tectonics of the South Island of New Zealand. Reviews of geophysics, 36(1):1-26.

Wapenaar, K. (2006). Green's function retrieval by cross-correlation in case of onesided illumination. Geophysical Research Letters, 33(19).

Wathelet, M., Jongmans, D., and Ohrnberger, M. (2004). Surface-wave inversion using a direct search algorithm and its application to ambient vibration measurements. Near surface geophysics, 2(4):211-221.

Wathelet, M., Jongmans, D., Ohrnberger, M., and Bonnefoy-Claudet, S. (2008). Array performances for ambient vibrations on a shallow structure and consequences over $\mathrm{V}_{S}$ inversion. Journal of Seismology, 12(1):1-19.

Wegler, U., Nakahara, H., Sens-Schönfelder, C., Korn, M., and Shiomi, K. (2009). Sudden drop of seismic velocity after the $2004 \mathrm{Mw} 6.6$ mid-Niigata earthquake, Japan, observed with passive image interferometry. Journal of Geophysical Research: Solid Earth, 114(B6).

Wegler, U. and Sens-Schönfelder, C. (2007). Fault zone monitoring with passive image interferometry. Geophysical Journal International, 168(3):1029-1033.

Wu, C., Delorey, A., Brenguier, F., Hadziioannou, C., Daub, E. G., and Johnson, 
P. (2016). Constraining depth range of S-wave velocity decrease after large earthquakes near Parkfield, California. Geophysical Research Letters, 43(12):6129-6136.

Xu, Z. J. and Song, X. (2009). Temporal changes of surface wave velocity associated with major Sumatra earthquakes from ambient noise correlation. Proceedings of the National Academy of Sciences, 106(34):14207-14212.

Yang, Y., Ritzwoller, M. H., Lin, F.-C., Moschetti, M., and Shapiro, N. M. (2008). Structure of the crust and uppermost mantle beneath the western United States revealed by ambient noise and earthquake tomography. Journal of Geophysical Research: Solid Earth (1978-2012), 113(B12).

Yang, Z., Sheehan, A. F., Collins, J. A., and Laske, G. (2012). The character of seafloor ambient noise recorded offshore New Zealand: Results from the MOANA ocean bottom seismic experiment. Geochemistry, Geophysics, Geosystems, 13(10):Q10011.

Yao, H., van Der Hilst, R. D., and Maarten, V. (2006). Surface-wave array tomography in SE Tibet from ambient seismic noise and two-station analysis - i. Phase velocity maps. Geophysical Journal International, 166(2):732-744. 